University of Tennessee Health Science Center

UTHSC Digital Commons

\title{
Failure of Primary Total Knee Arthroplasty: Establishing a Baseline for Retrieval Analysis Using Well-Functioning Necropsy Specimens
}

Meredith Perkins

University of Tennessee Health Science Center

Follow this and additional works at: https://dc.uthsc.edu/dissertations

Part of the Investigative Techniques Commons, Other Analytical, Diagnostic and Therapeutic Techniques and Equipment Commons, Surgical Procedures, Operative Commons, and the Therapeutics Commons

\section{Recommended Citation}

Perkins, Meredith (http://orcid.org/0000-0002-6492-0784), "Failure of Primary Total Knee Arthroplasty: Establishing a Baseline for Retrieval Analysis Using Well-Functioning Necropsy Specimens" (2018). Theses and Dissertations (ETD). Paper 460. http://dx.doi.org/10.21007/etd.cghs.2018.0451. 


\title{
Failure of Primary Total Knee Arthroplasty: Establishing a Baseline for Retrieval Analysis Using Well-Functioning Necropsy Specimens
}

\begin{abstract}
Introduction. Total knee arthroplasty (TKA) is an effective treatment for end-stage osteoarthritis of the knee. While many patients have positive functional and pain outcomes following primary TKA, a subset of patients have suboptimal outcomes, such as unexplained pain or aseptic loosening of the implant components necessitating revision of the index procedure. The understanding of the exact etiology of these suboptimal outcomes of primary TKA is in its infancy. In order to elucidate the etiology of failure in primary TKAs, a baseline for the factors contributing to arthroplasty failure and dissatisfaction must first be established. The purpose of this study was to investigate the relationship between soft tissue laxity, inflammatory cytokine concentrations, tissue metal concentrations, and wear scores of well-functioning implants retrieved from cadaveric specimens to determine the role of each of these factors in implant survivorship. It was hypothesized that decreased joint laxity would increase metal concentrations in the periprosthetic tissue, that cytokines specifically tied to the innate immune system would be elevated in laxer joints, and, lastly, that elevated inflammatory cytokines in the synovial fluid would be associated with elevated periprosthetic tissue metal concentrations.
\end{abstract}

Methodology. A total of 33 cadaveric specimens with primary TKA were obtained from two sources, the Medical Education and Research Institute (Memphis, TN) and RestoreLife USA (Elizabethton, TN), as part of institution review board approved multi-institutional orthopaedic retrieval program. Prior to testing and retrieval, fluoroscopically-assisted radiographs were taken to assess if any evidence of radiolucencies were present and all replacements were determined to be well-fixed per the images. Synovial fluid was then aspirated from the joint, processed, and stored in a $-80^{\circ} \mathrm{C}$ freezer. Each specimen was mounted into a custom knee testing platform and the IE rotation, varus-valgus (VV) deflection, and AP translation was measured at $0^{\circ}, 30^{\circ}, 60^{\circ}$, and $90^{\circ}$ of flexion. After collection of the laxity data, tissue samples were collected from the supra- and infra-patellar regions, the medial and lateral gutter, and from the tibia. The polyethylene inserts were assessed for wear on the condylar, backside, and stabilizing post surfaces, when applicable, and the femoral condyle was assessed for damage. A bead-based multiplex assay using the Luminex MAGPIX platform (R\&D Systems, Minneapolis, MN) was performed on the synovial aspirates for simultaneous detection of various inflammatory cytokines including: IL-1 $\beta$, IL-6, MCP-1, and MIP-3 $\alpha$. Inductively coupled plasma mass spectrometry (ICP-MS) was performed at Brooks Applied Labs (Bothel, WA) on the periprosthetic tissue samples for determination of tissue cobalt (Co), chromium (Cr), and titanium (Ti) concentrations. Finally, statistical analysis was conducted using SigmaPlot (Systat Software, Chicago, IL) to elucidate whether any correlations existed between the aforementioned factors.

Results. Decreased IE laxity at full extension was inversely correlated with increased Co concentrations in the periprosthetic tissues $(\rho=-0.64, p=0.02)$ with a sample size $(n)$ of 13 and a $95 \%$ confidence interval (Cl) from -0.88 to -0.14 . At $90^{\circ}$ of flexion, anterior laxity was inversely correlated with Co concentrations $(\rho=-0.66, p=0.03, n=11,95 \% \mathrm{Cl}:-0.90$ vi to -0.10$)$. At $60^{\circ}$ of flexion, anterior laxity was inversely correlated with $\mathrm{Cr}$ concentrations $(\rho=-0.63, \mathrm{p}=0.03, \mathrm{n}=13,95 \% \mathrm{Cl}:-0.88$ to -0.12$)$. Anterior laxity at $90^{\circ}$ of flexion was also inversely correlated with $\mathrm{Cr}$ concentrations ( $\rho=-0.81, p=0.003, n=11,95 \% \mathrm{Cl}:-0.95$ to -0.41$)$. Lastly, posterior laxity at $90^{\circ}$ of flexion was inversely correlated with $\mathrm{Cr}$ concentrations in the periprosthetic tissues $(\rho=-0.74, p=0.01, n=11,95 \% \mathrm{Cl}:-0.93$ to -0.25$)$. At $60^{\circ}$ of flexion, IE rotational laxity was inversely correlated with IL-1 $\beta$ concentrations $(\rho=-0.55, p=0.02, n=18,95 \% \mathrm{Cl}:-0.81$ to -0.11$)$. At $60^{\circ}$ of flexion, $\mathrm{VV}$ laxity was inversely correlated with TNF- $\alpha$ concentrations $(\rho=-0.78, p=2 \times 10-4, n=14,95 \% \mathrm{Cl}-0.93$ to -0.43). Additionally, VV laxity was inversely correlated with IL-1 $\beta$ concentrations $(\rho=-0.48, p=0.04, n=18$, $95 \% \mathrm{Cl}:-0.77$ to -0.02 ) at $60^{\circ}$ of flexion. At full extension, VV laxity was inversely correlated with IL-6 concentrations $(\rho=-0.46, p=0.04, n=19,95 \% \mathrm{Cl}$ : -0.76 to -0.01$)$. Anterior laxity was directly correlated with 
IL-6 concentrations $(\rho=0.53, p=0.02, n=18,95 \% \mathrm{Cl}: 0.08$ to 0.80$)$ at $90^{\circ}$ of flexion. Additionally, anterior laxity was directly correlated with MCP- 1 concentrations $(\rho=0.62, p=0.006, n=18,95 \% \mathrm{Cl}$ : 0.22 to 0.84$)$ at $90^{\circ}$ of flexion. Cr was inversely correlated with IL-6 ( $\rho=-0.52, p=0.01, n=21,95 \% \mathrm{Cl}:-0.78$ to -0.11$)$. Cr was also inversely correlated with MIP-3 $\alpha$ ( $\rho=-0.46, p=0.04, n=21,95 \% \mathrm{Cl}:-0.74$ to -0.04$)$.

Discussion. The specimens included in this study consisted of primary TKA implants that were retrieved at necropsy and were determined to be well-fixed per fluoroscopic analysis. The first objective of this study was to establish a baseline of different factors that likely contribute to failure of primary total knee replacements. This study provided semi-quantitative assessment of wear of the condylar surface, backside surface, and stabilizing post, when applicable, and of damage to the bearing surface of the femoral components. Joint laxity was measured using a custom knee testing platform that had been previously studied and utilized in published journal articles and theses. Inflammatory cytokine profiles in the synovial fluid samples were presented and measureable levels of metal debris was found in the periprosthetic tissue samples. The second objective of this study was to determine if any significant relationships arose in correlation analysis of the latter three aforementioned factors. An inverse trend was observed between joint laxity and tissue metal concentrations, such that decreased laxity induced metal release from the implant components. A direct trend was observed specifically between increased anteroposterior laxity and elevated inflammatory cytokines. Lastly, an inverse trend was found between decreased tissue metal concentrations and increased inflammatory cytokines. The third, and future, objective of this study is to utilize these measurements and observations for comparison with failed implants retrieved at time of revision. While some meaningful relationships were observed in this study, there were several limitations that must be noted. Firstly, this study had a relatively small sample size. Secondly, the cohort included a wide range of implants including cruciate-retaining, posterior-stabilized, fixed bearing, mobile bearing, monoblock, and modular designs fabricated from different materials

\section{Document Type}

Thesis

\section{Degree Name}

Master of Science (MS)

\section{Program}

Biomedical Engineering

\section{Research Advisor}

William M. Mihalko, MD, PhD

\section{Keywords}

Failure mechanisms, Inflammatory cytokines, Laxity, Tissue metal content, Total knee arthroplasty, Wear

\section{Subject Categories}

Analytical, Diagnostic and Therapeutic Techniques and Equipment | Investigative Techniques | Medicine and Health Sciences | Other Analytical, Diagnostic and Therapeutic Techniques and Equipment | Surgical Procedures, Operative I Therapeutics

\section{Comments}

Joint program with the University of Memphis. 
Failure of Primary Total Knee Arthroplasty: Establishing a Baseline for Retrieval Analysis Using Well-Functioning Necropsy Specimens

\author{
A Dissertation \\ Presented for \\ The Graduate Studies Council \\ The University of Tennessee \\ Health Science Center
}

\author{
In Partial Fulfillment \\ Of the Requirements for the Degree \\ Master of Science \\ In the Joint Graduate Program in Biomedical Engineering \\ From The University of Tennessee \\ and \\ The University of Memphis
}

By

Meredith Perkins

May 2018 
Copyright (c) 2018 by Meredith Perkins.

All rights reserved. 


\section{DEDICATION}

I would like to dedicate this research to the many people in my life that have supported me throughout my scholastic career. First and foremost, I would like to recognize my amazing parents, Maureen and Chris, for their unconditional love and support. Thank you for your encouragement, guidance, and patience. Without both of you, I would not be where I am today. I would also like to recognize and thank my brothers, Jack and Henry, for loving me despite my flaws and shortcomings. Both of you mean the world to me. To my grandparents, aunts, and uncles, thank you for always cheering me on and sending me words of encouragement, especially when I needed them most. To my entire family and my friends, thank you for listening to me obsess over my research and the knowledge I've gained in the past two years. I'm sure those conversations were not the most interesting or stimulating, but you allowed me to share my passion for research and learning. 


\section{ACKNOWLEDGEMENTS}

I would like to thank my advisor, Dr. William M. Mihalko, for the incredible opportunity to pursue a graduate degree in Biomedical Engineering. Thank you for your guidance and inspiration. Thank you for all the time you spent teaching me and pushing me to do better. Also, thank you for sparking my desire to pursue medicine and research. I would also like to thank my committee members, Dr. Richard Smith and Dr. John Williams, for their guidance, support, and feedback throughout these past two years.

I would like to thank Dr. John Williams, John Connor, David Knox, Devin Conner, Erik Woodard, Jason Lindsey, and other graduate students for their work developing and studying the knee machine and its data processing files. I would like to thank Allison Fetz, Benjamin Minden-Birkenmaier, and Dr. Gary Bowlin, their advisor, for allowing me to use their laboratory and equipment for the cytokine assay. Also, I would like to thank all of the students and researchers in Dr. Steven Kurtz's laboratory at Drexel University, especially Christina Arnholt, for their efforts in the various steps of this retrieval analysis study.

Finally, I would like to thank and acknowledge my classmates, who have become cherished friends. Leslie Pace and Allison Fetz, thank you for being my study partners over the past two years and thank you for your encouragement and hugs throughout the highs and lows of my graduate research. I would like to acknowledge and thank my predecessor, Julie Lowell, for being an incredible mentor and friend. Your positivity, kindness, and joy were contagious and made the office a much happier and enjoyable place. Thank you, Kylie Davis, for being my sarcastic counterpart in the office; the laughs we shared kept me going last year. Thank you to Griffin Heise for putting up with my neurotic and controlling tendencies; your mellow personality balanced out my highstrung, type A self. Lastly but certainly not least, a million thanks to Mrs. Anita Kerkhof, my "work mom." I truly cannot put into words how much I have enjoyed working with you and how much you have helped me grow throughout this program. I will miss working with each of you and cannot express my gratitude for each of your friendships during this journey. 


\begin{abstract}
Introduction. Total knee arthroplasty (TKA) is an effective treatment for end-stage osteoarthritis of the knee. While many patients have positive functional and pain outcomes following primary TKA, a subset of patients have suboptimal outcomes, such as unexplained pain or aseptic loosening of the implant components necessitating revision of the index procedure. The understanding of the exact etiology of these suboptimal outcomes of primary TKA is in its infancy. In order to elucidate the etiology of failure in primary TKAs, a baseline for the factors contributing to arthroplasty failure and dissatisfaction must first be established. The purpose of this study was to investigate the relationship between soft tissue laxity, inflammatory cytokine concentrations, tissue metal concentrations, and wear scores of well-functioning implants retrieved from cadaveric specimens to determine the role of each of these factors in implant survivorship. It was hypothesized that decreased joint laxity would increase metal concentrations in the periprosthetic tissue, that cytokines specifically tied to the innate immune system would be elevated in laxer joints, and, lastly, that elevated inflammatory cytokines in the synovial fluid would be associated with elevated periprosthetic tissue metal concentrations.
\end{abstract}

Methodology. A total of 33 cadaveric specimens with primary TKA were obtained from two sources, the Medical Education and Research Institute (Memphis, TN) and RestoreLife USA (Elizabethton, TN), as part of institution review board approved multiinstitutional orthopaedic retrieval program. Prior to testing and retrieval, fluoroscopically-assisted radiographs were taken to assess if any evidence of radiolucencies were present and all replacements were determined to be well-fixed per the images. Synovial fluid was then aspirated from the joint, processed, and stored in a $80^{\circ} \mathrm{C}$ freezer. Each specimen was mounted into a custom knee testing platform and the IE rotation, varus-valgus (VV) deflection, and AP translation was measured at $0^{\circ}, 30^{\circ}, 60^{\circ}$, and $90^{\circ}$ of flexion. After collection of the laxity data, tissue samples were collected from the supra- and infra-patellar regions, the medial and lateral gutter, and from the tibia. The polyethylene inserts were assessed for wear on the condylar, backside, and stabilizing post surfaces, when applicable, and the femoral condyle was assessed for damage. A bead-based multiplex assay using the Luminex MAGPIX platform (R\&D Systems, Minneapolis, $\mathrm{MN}$ ) was performed on the synovial aspirates for simultaneous detection of various inflammatory cytokines including: IL-1 $\beta$, IL-6, MCP-1, and MIP-3 $\alpha$. Inductively coupled plasma mass spectrometry (ICP-MS) was performed at Brooks Applied Labs (Bothel, WA) on the periprosthetic tissue samples for determination of tissue cobalt (Co), chromium (Cr), and titanium (Ti) concentrations. Finally, statistical analysis was conducted using SigmaPlot (Systat Software, Chicago, IL) to elucidate whether any correlations existed between the aforementioned factors.

Results. Decreased IE laxity at full extension was inversely correlated with increased Co concentrations in the periprosthetic tissues $(\rho=-0.64, p=0.02)$ with a sample size $(n)$ of 13 and a $95 \%$ confidence interval (CI) from -0.88 to -0.14 . At $90^{\circ}$ of flexion, anterior laxity was inversely correlated with Co concentrations $(\rho=-0.66, p=0.03, n=11,95 \%$ CI: -0.90 
to -0.10$)$. At $60^{\circ}$ of flexion, anterior laxity was inversely correlated with $\mathrm{Cr}$ concentrations $(\rho=-0.63, p=0.03, n=13,95 \%$ CI: -0.88 to -0.12$)$. Anterior laxity at $90^{\circ}$ of flexion was also inversely correlated with $\mathrm{Cr}$ concentrations $(\rho=-0.81, p=0.003, n=11$, $95 \%$ CI: -0.95 to -0.41$)$. Lastly, posterior laxity at $90^{\circ}$ of flexion was inversely correlated with $\mathrm{Cr}$ concentrations in the periprosthetic tissues $(\rho=-0.74, \mathrm{p}=0.01, \mathrm{n}=11,95 \% \mathrm{CI}$ : 0.93 to -0.25$)$. At $60^{\circ}$ of flexion, IE rotational laxity was inversely correlated with IL-1 $\beta$ concentrations $(\rho=-0.55, p=0.02, n=18,95 \%$ CI: -0.81 to -0.11$)$. At $60^{\circ}$ of flexion, VV laxity was inversely correlated with TNF- $\alpha$ concentrations $\left(\rho=-0.78, p=2 \times 10^{-4}, n=14\right.$, $95 \%$ CI -0.93 to -0.43 ). Additionally, VV laxity was inversely correlated with IL-1 $\beta$ concentrations $(\rho=-0.48, p=0.04, n=18,95 \%$ CI: -0.77 to -0.02$)$ at $60^{\circ}$ of flexion. At full extension, VV laxity was inversely correlated with IL- 6 concentrations $(\rho=-0.46, p=0.04$, $\mathrm{n}=19,95 \% \mathrm{CI}:-0.76$ to -0.01$)$. Anterior laxity was directly correlated with IL-6 concentrations $(\rho=0.53, p=0.02, n=18,95 \%$ CI: 0.08 to 0.80$)$ at $90^{\circ}$ of flexion. Additionally, anterior laxity was directly correlated with MCP-1 concentrations $(\rho=0.62$, $\mathrm{p}=0.006, \mathrm{n}=18,95 \% \mathrm{CI}: 0.22$ to 0.84 ) at $90^{\circ}$ of flexion. $\mathrm{Cr}$ was inversely correlated with IL-6 $(\rho=-0.52, p=0.01, n=21,95 \%$ CI: -0.78 to -0.11$)$. Cr was also inversely correlated with MIP-3 $\alpha(\rho=-0.46, p=0.04, n=21,95 \%$ CI: -0.74 to -0.04$)$.

Discussion. The specimens included in this study consisted of primary TKA implants that were retrieved at necropsy and were determined to be well-fixed per fluoroscopic analysis. The first objective of this study was to establish a baseline of different factors that likely contribute to failure of primary total knee replacements. This study provided semi-quantitative assessment of wear of the condylar surface, backside surface, and stabilizing post, when applicable, and of damage to the bearing surface of the femoral components. Joint laxity was measured using a custom knee testing platform that had been previously studied and utilized in published journal articles and theses. Inflammatory cytokine profiles in the synovial fluid samples were presented and measureable levels of metal debris was found in the periprosthetic tissue samples. The second objective of this study was to determine if any significant relationships arose in correlation analysis of the latter three aforementioned factors. An inverse trend was observed between joint laxity and tissue metal concentrations, such that decreased laxity induced metal release from the implant components. A direct trend was observed specifically between increased anteroposterior laxity and elevated inflammatory cytokines. Lastly, an inverse trend was found between decreased tissue metal concentrations and increased inflammatory cytokines. The third, and future, objective of this study is to utilize these measurements and observations for comparison with failed implants retrieved at time of revision. While some meaningful relationships were observed in this study, there were several limitations that must be noted. Firstly, this study had a relatively small sample size. Secondly, the cohort included a wide range of implants including cruciate-retaining, posterior-stabilized, fixed bearing, mobile bearing, monoblock, and modular designs fabricated from different materials. 


\section{TABLE OF CONTENTS}

CHAPTER 1. INTRODUCTION .....................................................................................1

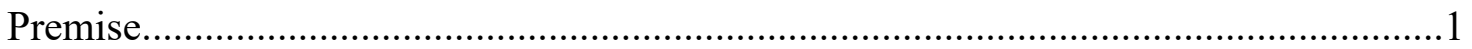

Functional Anatomy and Kinematics of the Natural Knee.......................................... 1

Grood and Suntay Joint Coordinate System .................................................................

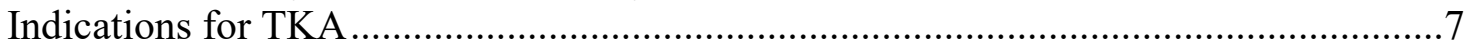

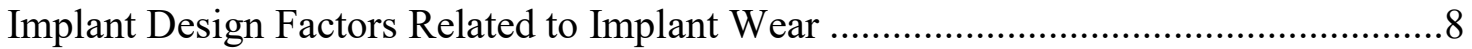

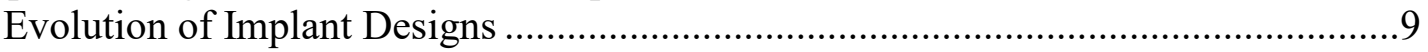

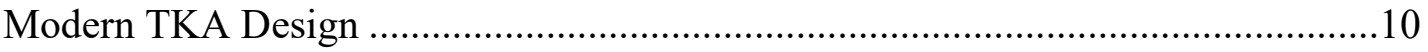

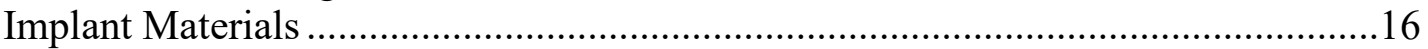

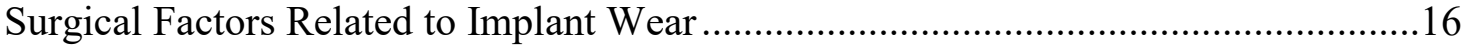

Proper Alignment of Components to Restore a Reference Axis ..............................16

Recreation of Soft Tissue Gaps in Flexion and Extension.......................................20

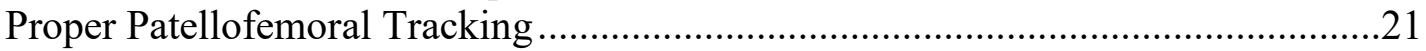

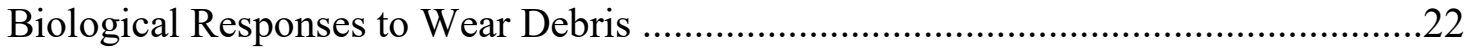

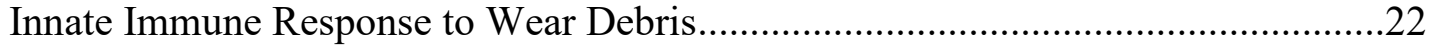

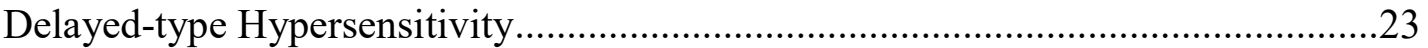

Adverse Local Tissue Reactions ......................................................................23

Systemic Effects and Associated Toxicity of Metallic Debris ................................24

Inflammatory Cytokines as Biomarkers of Inflammation and Osteolysis...................26

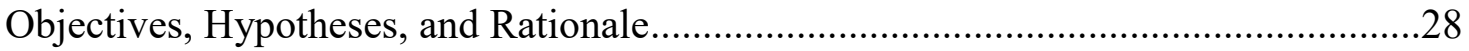

CHAPTER 2. METHODOLOGY .....................................................................30

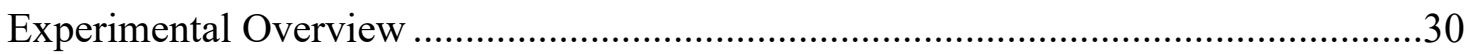

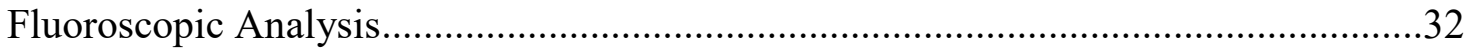

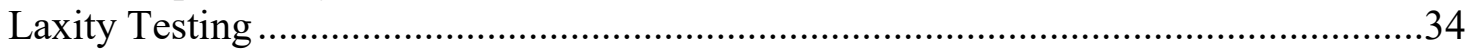

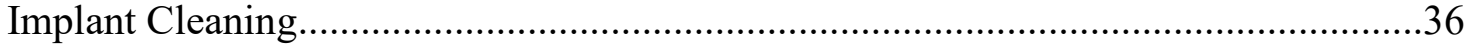

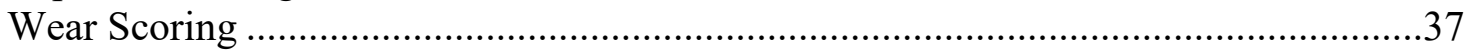

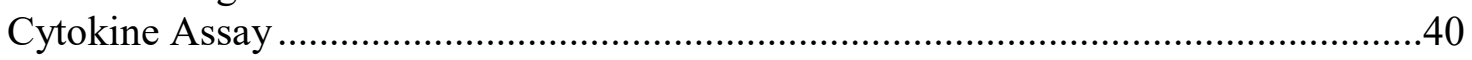

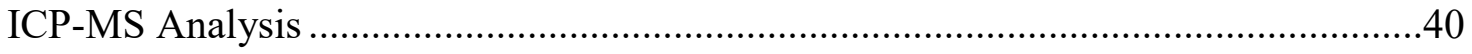

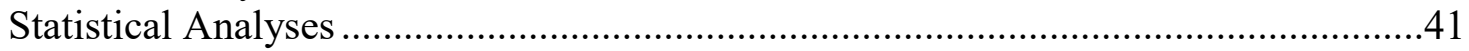

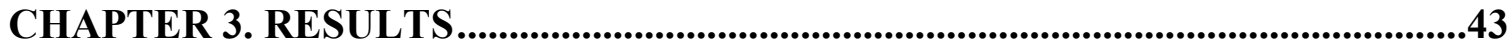

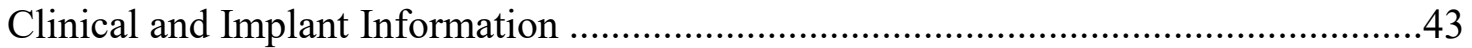

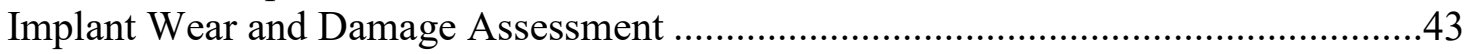

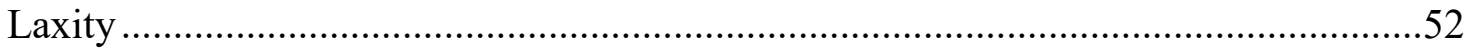

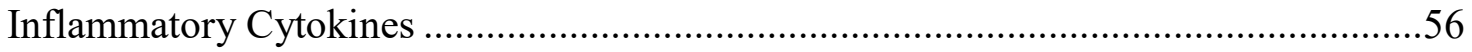

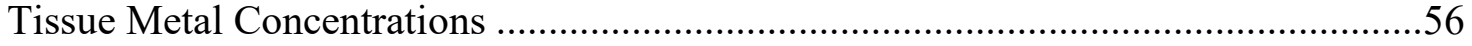

Correlation of Study Parameters ..............................................................................59

Hypothesis (a) - Laxity and Tissue Metal Concentrations ........................................59

Hypothesis (b) - Laxity and Inflammatory Cytokines..........................................61

Hypothesis (c) - Tissue Metal Concentrations and Inflammatory Cytokines ...........64 
CHAPTER 4. DISCUSSION .......................................................................................65

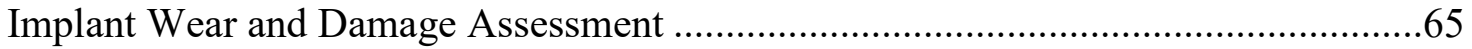

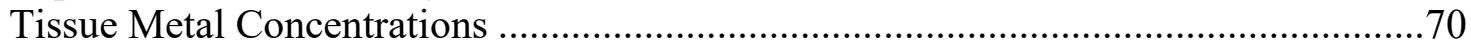

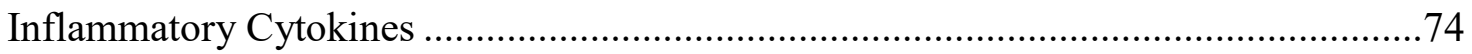

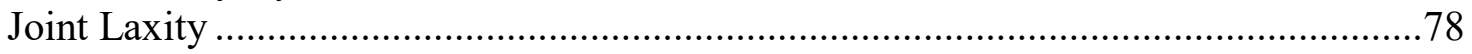

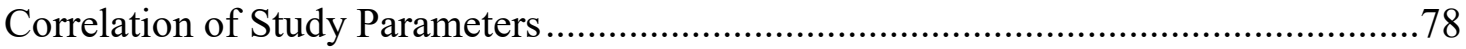

Hypothesis (a) - Laxity and Tissue Metal Concentrations ........................................78

Hypothesis (b) — Laxity and Inflammatory Cytokines..............................................86

Hypothesis (c) - Tissue Metal Concentrations and Inflammatory Cytokines ............88

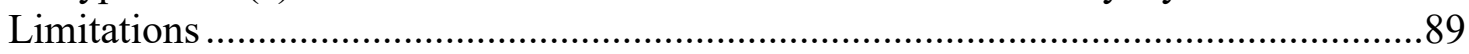

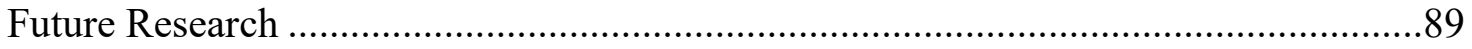

LIST OF REFERENCES .................................................................................................91

APPENDIX A. FLUOROSCOPIC ANALYSIS OF POST MORTEM

RETRIEVED IMPLANTS................................................................................................109

APPENDIX B. CORRELATIONS BETWEEN LAXITY AND TISSUE METAL

CONCENTRATIONS ..........................................................................................126

APPENDIX C. CORRELATIONS BETWEEN LAXITY AND

INFLAMMATORY CYTOKINES ...................................................................................139

APPENDIX D. CORRELATIONS BETWEEN TISSUE METAL

CONCENTRATIONS AND INFLAMMATOR CYTOKINES ...................................164

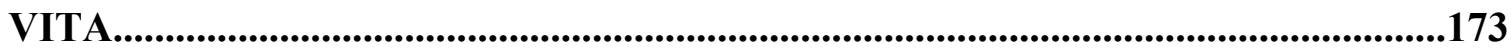




\section{LIST OF TABLES}

Table 2-1. Summary of the polyethylene wear score quantification as proposed by

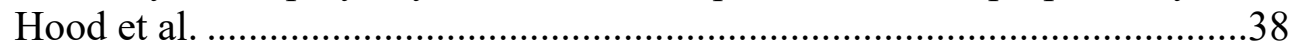

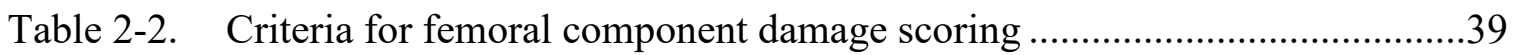

Table 3-1. Implant information of study cohort including manufacturer, design, and

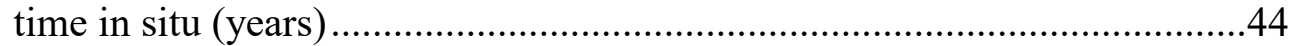

Table 3-2. Summary of total tibial insert wear scores by location .............................45

Table 3-3. Summary of internal and external rotational laxity at each flexion angle

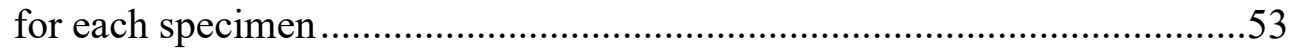

Table 3-4. Summary of varus and valgus laxity at each flexion angle for each specimen

Table 3-5. Summary of anterior and posterior laxity at each flexion angle for each specimen

Table 3-6. Concentrations of inflammatory cytokines for cadaveric specimens $(\mathrm{pg} / \mathrm{mL})$

Table 3-7. Periprosthetic tissue metal concentrations reported in $(\mu \mathrm{g} / \mathrm{L})$

Table 3-8. Spearman correlation coefficients $(\rho)$ for IE rotational laxity and periprosthetic tissue metal concentrations

Table 3-9. Spearman correlation coefficients $(\rho)$ for VV laxity and periprosthetic tissue metal concentrations.

Table 3-10. Spearman correlation coefficients $(\rho)$ for AP laxity and periprosthetic tissue metal concentrations.

Table 3-11. Spearman correlation coefficients $(\rho)$ for IE laxity and inflammatory cytokine concentrations in the synovial fluid.....

Table 3-12. Spearman correlation coefficients $(\rho)$ for VV laxity and inflammatory cytokine concentrations in the synovial fluid

Table 3-13. Spearman correlation coefficients $(\rho)$ for AP laxity and inflammatory cytokine concentrations in the synovial fluid.

Table 3-14. Spearman correlation coefficients $(\rho)$ for tissue metal concentrations and inflammatory cytokine concentrations in the synovial fluid with outliers removed 
Table 4-1. Compilation of previous studies investigation metal concentrations from TKA for comparison for current study 


\section{LIST OF FIGURES}

Figure 1-1. Anatomical stabilizing structures of knee joint .........................................

Figure 1-2. Motions of the knee as defined by the Grood and Suntay (1) joint

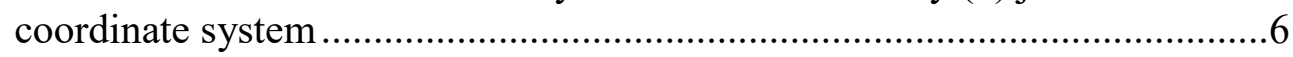

Figure 1-3. Total Knee Arthroplasty components .................................................17

Figure 1-4. Specimen RLU0426161B-L, retrieved in this study, presented with metallosis .25

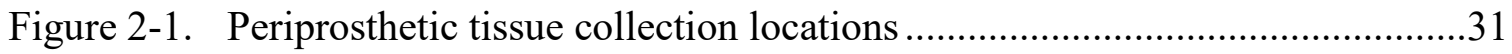

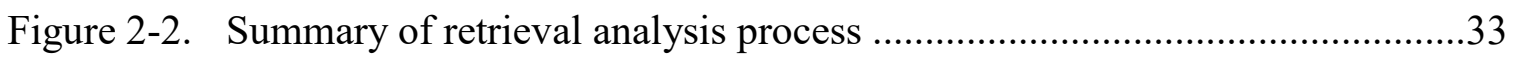

Figure 2-3. Custom knee testing platform in Dr. William Mihalko's lab ......................35

Figure 2-4. Technique used to divide the bearing and backside surface of tibial insert

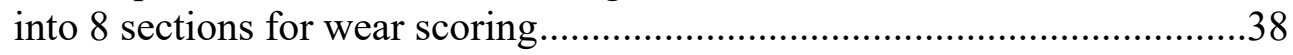

Figure 2-5. Scoring method for the femoral component. .........................................

Figure 3-1. Specimen RLU0426162B-R tibial insert ................................................46

Figure 3-2. Specimen RLU1028161B-L tibial insert ............................................48

Figure 3-3. Specimen RLU1121162A-R tibial insert............................................49

Figure 3-4. Specimen RLU0829162C-R femoral component ....................................50

Figure 3-5. Specimen RLU1028161B-L femoral component ....................................51

Figure 4-1. IE rotational laxity comparison between the present study and Saeki et al., Kanamiya et al., and Jojima et al......................................................79

Figure 4-2. VV laxity comparison between the present study and Saeki et al. and Jojima et al.

Figure 4-3. Comparison of measured AP laxity between current study, Saeki et al. and Jojima et al.

Figure 4-4. Comparison of IE laxity between current study and Woodard. .82

Figure 4-5. Comparison of VV laxity between current study and Woodard. .83

Figure 4-6. Comparison of AP laxity between current study and Woodard .84 


\section{LIST OF ABBREVIATIONS}

ACL

Al

ALTR

ALVAL

AP

Co

CR

$\mathrm{Cr}$

DTH

GM-CSF

IE

IFN

IL

IQR

JCS

LCL

MCL

MCP

$\mathrm{M}-\mathrm{CSF}$

MIP

MMP

Mo

$\mathrm{OA}$

OPG

PCL

PMMA

PRR

PS

RA

RANK

RANKL

ROM

THA

$\mathrm{Ti}$

TJR

TKA

TNF

UHMWPE

$\mathrm{V}$

VV
Anterior cruciate ligament

Aluminum

Adverse local tissue reaction

Aseptic lymphocytic vasculitis-associated lesions

Anterior-posterior

Cobalt

Cruciate-retaining

Chromium

Delayed-type hypersensitivity

Granulocyte macrophage-colony stimulating factor

Internal-external

Interferon

Interleukin

Interquartile range

Joint coordinate system

Lateral collateral ligament

Medial collateral ligament

Monocyte chemotactic protein

Macrophage-colony stimulating factor

Macrophage inflammatory protein

Matrix metalloproteinase

Molybdenum

Osteoarthritis

Osteoprotegerin

Posterior cruciate ligament

Polymethylmethacrylate

Pattern recognition receptor

Posterior-stabilized

Rheumatoid arthritis

Receptor activator of nuclear factor kappa B

Receptor activator of nuclear factor kappa B ligand

Range of motion

Total hip arthroplasty

Titanium

Total joint replacement

Total knee arthroplasty

Tumor necrosis factor

Ultra-high molecular weight polyethylene

Vanadium

Varus-valgus 


\section{CHAPTER 1. INTRODUCTION}

\section{Premise}

Total joint replacements (TJR) are an effective treatment for end-stage osteoarthritis and are among the most commonly performed operations in the United States, with approximately 1 million procedures per year (1). An estimated 332,000 total hip arthroplasties (THA) and 719,000 total knee arthroplasties (TKA) occurred in 2010 alone, with $10-15 \%$ of these operations being revision procedures (1). The number of primary TKAs is projected to grow $673 \%$ to 3.48 million procedures by the year 2030 whereas the number of revision TKAs is projected to grow by $601 \%$ between the years 2005 and 2030, from 38,300 to 268,200 annual procedures (2). Increasing incidence of primary TKAs is likely due to increasing longevity of the population, decreasing age at arthroplasty, and excellent pain and functional outcomes associated with these procedures.

A number of patients, however, have suboptimal outcomes as indicated by increasing number of revisions or from patient reported outcome measures. Only $85 \%$ of patients reported satisfaction following primary TKA (3). Up to $20 \%$ of patients report long-term pain after TKA, which is dramatically more than that which is reported following THA (4-6). Common causes of failure include: aseptic component loosening, infection, ligament/flexion instability, periprosthetic fracture, arthrofibrosis, patellofemoral maltracking, abnormal joint line problems, and metal hypersensitivity (7). Oftentimes, failure of an index procedure is multifactorial; Mulhall et al. (8) analyzed the etiologies of failure in 318 knee replacements and found that $64.4 \%$ had more than one identifiable cause. Clinically, revision arthroplasty is a complex procedure associated with higher complication rates, extended hospitalization, unsatisfactory functional outcomes, and relatively shorter implant survival compared to primary procedures (7). In order to elucidate the etiology of failure in primary TKAs, a baseline for the factors contributing to arthroplasty failure and dissatisfaction must first be established. The purpose of this study was to investigate the relationship between soft tissue laxity, inflammatory cytokine concentrations, tissue metal concentrations, and wear scores of well-functioning implants retrieved from cadaveric specimens to determine the role of each of these factors in implant survivorship.

\section{Functional Anatomy and Kinematics of the Natural Knee}

The knee is one of the largest and most complex joints in the body. It is composed of three compartments with different articulations: the patellofemoral joint and the medial and lateral tibiofemoral joint. The patellofemoral joint is the articulation between the patella and the femoral trochlear groove. The patella is covered with articular cartilage and articulates with the trochlear groove of the femur. The primary function of the patella is to increase the lever arm of the extensor mechanism around the knee, improving the efficiency of quadriceps contraction (9). The knee extensor mechanism is a complex 
structure formed by the quadriceps femoris muscle group, quadriceps femoris tendon, patella, patellar tendon, retinaculum, and surrounding soft tissue structures that help stabilize the patellofemoral joint and the knee. The quadriceps femoris tendon connects the rectus femoris, vastus lateralis, vastus medialis, and vastus intermedius, which make up the quadriceps femoris, to the patella. Continuous with the quadriceps femoris tendon is the patellar ligament that connects from the patella to the tibial tuberosity. The quadriceps femoris tendon and patellar ligament transmit tensile forces generated by the quadriceps to the tibia. The patella is stabilized in the middle of the knee by the medial and lateral retinaculum. Patellofemoral stability is maintained by a combination of the articular surface geometry and soft-tissue restraints.

The tibiofemoral joint functions to transmit body weight from the femur to the tibia. On the distal end of the femur are two rounded condyles that project posteriorly beyond the shaft, forming a J-shaped curve that guides movement of the joint. The femoral and tibial condyles are covered with articular cartilage which protects the bone and enables easy articulation at the joint. The menisci, two C-shaped layers of fibrocartilage, are located between the articulating surfaces and act as shock absorbers that cushion the joint. All remaining surfaces of the knee are in capsular attachment and are covered by the synovial membrane that secretes the synovial fluid which is responsible for cartilage nutrition, the removal of metabolites from the joint, joint lubrication, and the provision of immune cells that are involved in the removal of foreign materials and organisms from the joint (10). Stability of the tibiofemoral joint is dependent upon many static and dynamic elements. The primary static stabilizers are the ligaments of the knee: the anterior cruciate ligament (ACL), posterior cruciate ligament (PCL), medial collateral ligament (MCL), and lateral collateral ligament (LCL). All of these structures, shown in Figure 1-1, work in concert to provide stability and painless function in a healthy knee joint.

Knee motion is much more complex than simple flexion and extension about a fixed transverse axis like a simple hinge; instead, knee motion during normal gait occurs in three separate planes and is referred to as "triaxial motion"(9). Flexion of the knee, which occurs about a constantly changing center of rotation, is a function of the articular geometry of the knee and the ligamentous restraints (9). Freeman et al. (11) described sagittal plane condylar geometry as a sequence of arcs with different radii that interact with a flat medial and convex lateral tibial plateau. In a study of in vivo knee kinematics using fluoroscopic video surveillance with three-dimensional computed tomography (CT) scans, Dennis et al. (12) described this flexion axis as varying in a helical fashion in a normal knee. When plotted, the path of this changing center of rotation describes a $\mathbf{J}$ shaped curve that mirrors the sagittal curvature of the femoral condyles.

Flexion is achieved by a complex coupled mechanism in which the femoral condyles simultaneously glide and roll back on the tibial plateaus about multiple simultaneous axes, known as "posterior rollback". In an in vivo fluoroscopic analysis of normal human gait, Komistek et al. (13) found that posterior rollback was substantially greater at the lateral femorotibial articulation than medially, thereby creating a medial pivot type of axial rotational pattern in which the tibia internally rotates relative to the 


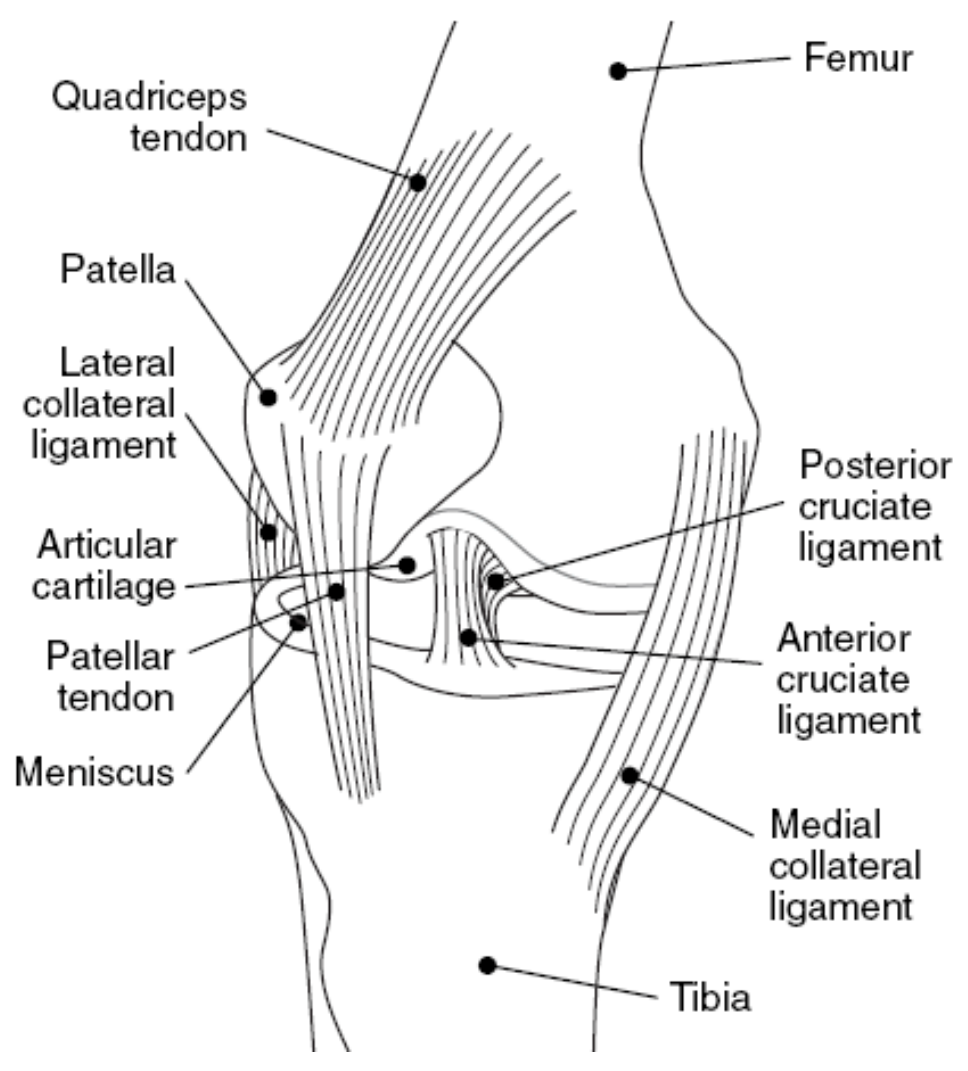

Figure 1-1. Anatomical stabilizing structures of knee joint

Reprinted with permission. National Institute of Arthritis and Musculoskeletal and Skin Diseases. Medial view of the knee showing anatomical features. May 2001. Accessed 18 August 2017. 
femur as flexion increases. At full extension, the femur is internally rotated with respect to the tibia and is located anteriorly to the mid-point of the tibial plateau. As the knee flexes, the lateral condyle of the femur consistently translates posteriorly on the lateral tibial plateau with increasing flexion $(12,13)$. On the other hand, the medial condyle has a less consistent motion pattern that includes posterior translation and anterior slide during knee flexion, which leads to a reduced overall posterior translation of the medial condyle on the tibial plateau. Because the medial tibial plateau articular surface is longer than the lateral tibial plateau surface, extension is achieved by $5^{\circ}$ external rotation of the tibia in the last $15^{\circ}$ of extension in the "screw home" mechanism, which results in the tightening of both cruciate ligaments, locking the knee in place providing maximal stability with respect to the femur and decreasing the work performed by the quadriceps femoris while standing. This pattern of medially based pivoting of the knee explains the observed external rotation of the tibia on the femur during extension, known as the "screw-home mechanism," and the internal rotation of the tibia during knee flexion (9).

\section{Grood and Suntay Joint Coordinate System}

A thorough understanding of joint kinematics is important in the diagnosis of joint disorders resulting from injury or disease, in the quantitative assessment of treatment, in the design of better prosthetic devices, and in the general study of gait (14). However, in 1983, two researchers found that the traditional characterization of motion was not easily understood by clinicians, whose understanding of such material is pivotal to proper diagnosis and treatment (14). To bridge the understanding and communication of knee kinematics between physicians and biomechanical engineers, Grood and Suntay (14) sought to establish a basis for description and measurement of the six motion components in the experimental study of knee joint kinematics.

A joint coordinate system (JCS) is a reference system for a particular joint (the knee) in relation to global coordinate systems of the whole body (the anatomic axes) and in relation to local coordinate systems of other joints. The relative motion of two body segments, such as the distal end of the femur and the proximal end of the tibia, can be defined by a JCS, which is described by two segment-fixed axes and a mutually orthogonal floating axis. Angular rotation of these bodies is about one or more of these spatial axes. In developing a JCS for the knee, Grood and Suntay (14) stated that is first necessary to specify: 1) the Cartesian coordinate system fixed in each bone used to describe its shape; 2) the body-fixed axes and the reference axes of the JCS used to describe the relative motion between the two bones; and 3) the location of the translation reference point. The Grood and Suntay JCS served as convention behind the methods used to quantify knee kinematics and their relationship to joint laxity in this study.

The z-axis, along the tibial mechanical axis, was selected as the tibial body fixed axis and was defined as a vector extending from the origin at the center of the medial and lateral malleoli to the intercondylar eminence between the medial and lateral tibial condyles. The z-axis is deemed positive upwards from the origin and negative downwards towards the origin. The tibial y-axis was selected as the reference axis and 
was oriented anteriorly, which was defined as the cross-product of the fixed axis (z-axis) with a line connecting the approximate center of each plateau. The third and final axis of the tibial body fixed system, the x-axis, was obtained by the cross-product of the reference axis (y-axis) and the fixed axis (z-axis), such that the $\mathrm{x}$-axis is positive to the right and, therefore, oriented laterally in the right knee and medially in the left knee. The femoral body fixed system was specified to originate from intercondylar notch between the medial and lateral femoral condyles. The Z-axis, along the femoral mechanical axis, was defined as a vector extending from this origin at the intercondylar notch to the center of the femoral head. The femoral Y-axis was selected as the reference axis and was oriented anteriorly, as defined by the cross product of the femoral body fixed axis (Zaxis) with a line connecting the two points on the posterior surface of the femoral condyles when the knee was in full extension. Finally, X-axis was selected as the femoral body fixed axis and was obtained by the cross-product of the reference axis ( $Y$ axis) and the Z-axis. In summary, the JCS of the knee was composed of two body fixed axes: the tibial $\mathrm{z}$-axis and the femoral $\mathrm{X}$-axis, and one floating axis as defined by the cross-product of the two body fixed axes that is positive in the anterior direction.

Once the body fixed systems and JCS axes were established, the relative joint rotations between the bones then could be defined with reference to the JCS, as depicted in Figure 1-2. Flexion/extension occurred about the femoral body fixed axis, the X-axis. The angle of flexion was defined as the angle between the floating axis and the femoral reference axis, the $\mathrm{Y}$-axis. Internal/external rotation occurred about the tibial body fixed axis, the $\mathrm{z}$-axis. The angle of internal/external rotation was defined as the angle between the floating axis and the tibial reference axis, the y-axis. Abduction/adduction occurred about the floating axis. The angle of abduction/adduction or the varus/valgus deflection angle is the angle formed between the femoral body fixed axis, the X-axis, and the tibial body fixed axis, the $z$-axis, minus $\pi / 2$, so that the varus/valgus angle will be set to $0^{\circ}$ in full extension. Regardless whether the knee in question was right or left, flexion was defined as positive and extension as negative. For the other rotations, the sign conventions differed between right and left knees because the tibial $\mathrm{x}$-axis was always designated positive to the right. In a right knee, external rotation and abduction (or varus deflection) of the tibia with respect to the femur was deemed positive, while internal rotation and adduction (valgus deflection) was deemed negative. In a left knee, these conventions were reversed such that external rotation and abduction (varus deflection) were negative while internal rotation and adduction (valgus deflection) were positive.

Grood and Suntay (14) also described the three translational motions of the joint: medial/lateral, anterior/posterior, and axial. They defined these three joint translations by a vector extending between the tibial and femoral origins with vector components defined within the JCS. The three components of the translational vector were designated by $\mathrm{S}_{1}$, $\mathrm{S}_{2}$, and $\mathrm{S}_{3}$. Used to determine the medial/lateral translational motion, $\mathrm{S}_{1}$ was defined as the distance from the femoral origin along the femoral body fixed axis, the X-axis, to the intersection of the $\mathrm{X}$-axis and the floating axis. $\mathrm{S}_{2}$, which was used to determine the anterior/posterior translation of the tibia with respect to the femur, was defined as the distance between the femoral body fixed axis, the $\mathrm{X}$-axis, and the tibial body fixed axis, the z-axis, along their common perpendicular, the floating axis. Grood and Suntay (14) 


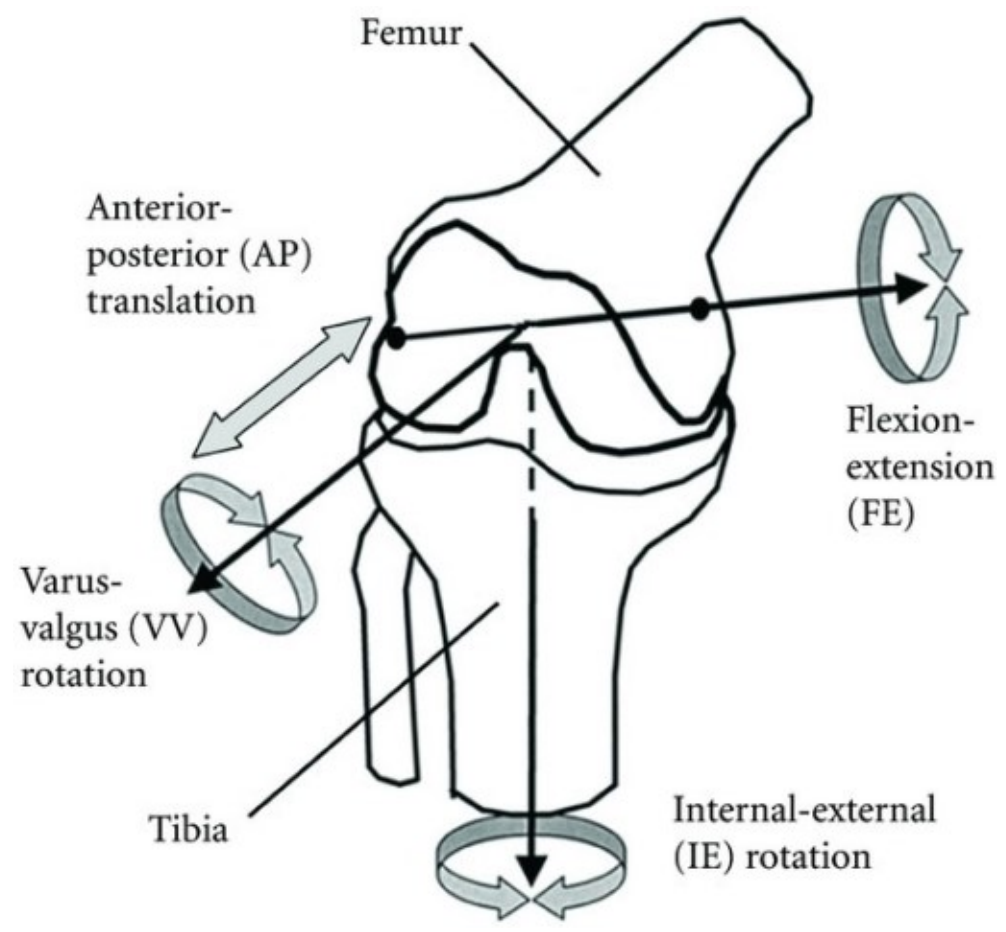

Figure 1-2. Motions of the knee as defined by the Grood and Suntay (1) joint coordinate system

Reprinted with permission. Amiri S, Wilson DR. A computational modeling approach for investigating soft tissue balancing in bicruciate retaining knee arthroplasty. Comput Math Methods Med; 2012. 
designated anterior translation of the tibia with respect to the femur as positive while posterior translation was negative. Lastly, $\mathrm{S}_{3}$, which determined the axial motion of the joint (i.e. the vertical motion), was defined as the distance between the tibial origin along the tibial body fixed axis, the z-axis, to the intersection of the z-axis and the floating axis.

\section{Indications for TKA}

In a healthy knee, all of the aforementioned structures facilitate painless function of the knee joint. However, there are many arthritic conditions that destroy or damage one or more of the joint structures causing pain, muscle weakness, and reduced function, prompting many to seek replacement surgery. Osteoarthritis (OA) is a degenerative disease in which the articular cartilage is worn away. This is caused by a disruption in the factors - such as inflammatory cytokines and proteases - that regulate the dynamic equilibrium between the continual, ongoing formation and breakdown of cartilaginous matrix. When the catabolic influences exceed the anabolic influences, chondrocytes can no longer compensate for the continuous breakdown of the matrix and gradual degradation occurs. As the cartilage degrades, bone on bone articulation results in the formation of osteophytes and narrowing of the joint space causing pain and stiffness. Post-traumatic arthritis is a form of OA that occurs after a traumatic injury to the knee joint, such as a fracture or ligament tear. These injuries often result in chronic inflammation or affect the natural alignment of the knee, gradually damaging the articular cartilage. Often, those who suffer trauma at a young age are susceptible to developing arthritis earlier than those who naturally develop OA (15). Rheumatoid arthritis (RA) is a chronic systemic autoimmune disease of unknown cause that primarily involves joints. In RA, the immune system attacks the synovial membrane, causing it to become chronically inflamed and thickened, which can damage the cartilage eventually leading to cartilage loss. Like OA, RA is believed to be mediated by cytokines and matrix metalloproteinases (16).

The primary indication for TKA is to relieve pain caused by severe arthritis, with or without significant deformity. Persons with severe arthritis may report joint pain, stiffness, and difficulty with mobility; oftentimes to the point that the reduced function interferes with activities of daily living and compromises the quality of life (17). Other sources of knee and leg pain must be sought and systematically excluded. Radiographic findings must correlate with a clear clinical impression of knee arthritis. The classification for OA described by Kellgren and Lawrence is the most widely used radiological classification to identify and grade OA. Kellgren and Lawrence defined the progression of OA in five grades ( 0 , normal, to 4 , severe) and combined it with important radiological changes evident at each stage including: formation of osteophytes on the joint margins or in ligamentous attachments; narrowing of the joint space associated with sclerosis of subchondral bone; cystic areas with sclerotic walls situated in the subchondral bone; and altered shape of bone ends $(18,19)$. Typically, patients with Kellgren and Lawrence Grade 3 or 4 OA qualify for TKA. Furthermore, conservative treatment measures should be exhausted prior to considering surgery. Conservative measures may include: patient education and lifestyle modification, rehabilitation through 
physical therapy, anti-inflammatory medications, and intraarticular injections $(9,20)$. Several studies have found an inverse relationship between severity of OA based on preoperative radiographs and satisfaction following TKA (21-25). More plainly, performing a TKA on patients with early-grade arthritis may put those patients at a higher risk for pain and dissatisfaction. As such, these guidelines are essential in ensuring optimal pain and function outcomes for patients following primary TKA.

While the primary indication for TKA is to relieve pain and restore function in patients suffering from severe arthritis, deformity can become the principal indication for arthroplasty in patients with moderate arthritis and variable levels of pain when the progression of deformity begins to threaten the expected outcome of an anticipated arthroplasty (9). Flexion contracture, or stiffness upon extension, is a common deformity encountered in TKA (26). When fully extended, the knee can bear weight without constant muscle energy expenditure. In patients with a flexion contracture, a large amount of energy is needed from the quadriceps to help the knee bear load and remain stable. This causes standing, walking, and stair climbing to be abnormally tiring, reducing overall knee flexion $(27,28)$. For contractures of $10^{\circ}$ or more, particular techniques, performed in a specified order, may be necessary to correct the flexion contracture. However, previous studies have indicated that patients with large flexion contractures preoperatively, greater than $20^{\circ}$, are more likely to experience a flexion contracture postoperatively $(27,29)$. Similarly, excessive varus or valgus laxity is a deformity that poses a risk of compromising the outcome of arthroplasty as its severity progresses. A varus knee has displacement of the distal tibia toward the midline of the body in the coronal plane, in which the mechanical axis of the limb lies medially, resulting in a "bow-legged" appearance. On the other hand, a valgus knee occurs when the distal tibia rotates away from the midline, in which the mechanical axis of the limb lies laterally, resulting in a "knock-kneed" appearance. As the severity of the varus or valgus deformity progresses, a constrained condylar type of prosthesis may be necessary to prevent subsequent instability in the coronal plane. Complications of varus/valgus constrained designs have been well documented and result from the large stressed transmitted to the highly constrained implant (30-32). Intervening before this degree of laxity is reached allows the use of a prosthesis that lacks coronal plane constraint and has a more favorable expected survivorship (9).

\section{Implant Design Factors Related to Implant Wear}

TKA is a safe and efficacious operation that relieves pain and improves function $(1,2,33)$. Originally, this operation was intended for elderly patients with severe endstage arthritis of the knee. However, more recently, TKA has been extended to younger, more active patients with higher demands in their lifestyles. Kurtz et al. (34) projected that by 2030, patients under the age of 65 could account for $55 \%$ of the demand for primary TKA; furthermore, this data suggests that growth in use of TKA occurs in patients primarily between the ages of 45 to 65 . These patients often voice high expectations prior to surgery with respect to restoration of function and even return to leisure activities; leisure activities range between low intensity, such as walking or 
bowling, to high intensity, such as basketball or snow skiing (35). Regardless of the expectations of elderly or younger patients, the base requirement of a TKA is to restore basic knee function.

To accomplish this goal, the TKA must replicate normal knee motion characteristics. Many design variations of the femoral components and tibial inserts have been proposed to achieve this elusive feat. However, every TKA has a finite lifespan as demonstrated by the projection that patients below the age of 65 that have received primary TKA could account for $62 \%$ of the demand for revision TKA by 2030 (34). This lifespan is dependent upon the ability of the implant materials to withstand the physical demands of the patient. Successful outcomes as well as implant longevity following primary TKA are believed to be dependent on implant-specific factors including the design, materials, and the manufacturing or sterilization technique. This section will provide an overview of the evolution of TKA prostheses to the current designs employed, the materials utilized, and how each factor may influence wear of the implant.

\section{Evolution of Implant Designs}

The first attempt of treating patients affected by knee arthropathies with joint replacement dates back to the mid-nineteenth century. In 1860, Verneuil proposed interpositional arthroplasty and attempted to build an artificial knee using the articular capsule to recreate the joint. In 1880, the German surgeon, Thermistocles Gluck, imagined the first primitive hinge joints made of ivory. However, despite his proposed system of joint replacement, the first part of the twentieth century only used interpositional arthroplasty using autologous tissues or metallic surfaces placed between the articulating surfaces of the joint. In the 1950s, Walldius developed a hinged prosthesis that replaced the joint surfaces of the femur and tibia (36). In the mid-to-late 1960s, Gunston developed polycentric knee arthroplasty by adopting the concepts of low friction replacements proposed by Charnley; additionally, he introduced the first cemented surface arthroplasty of the knee joint (37).

In the late 1960s, Drs. Freeman and Swanson were the first to approach condylar total knee design from a predominantly functional perspective (38). In 1970, Drs. Walker, Insall, Ranawat, and Inglis, at the Hospital for Special Surgery in New York, City, combined their respective design and surgical experienced to develop the duocondylar prosthesis. The Duocondylar was an anatomical, symmetric, cemented knee that preserved both cruciate ligaments (39). The lessons learned from the Duocondylar prosthesis design led to the development of the second generation of designs to come from the Hospital for Special Surgery - the anatomically designed duopatellar prosthesis and the functionally design total condylar prosthesis (40).

Simultaneous with the development of the Insall et al. (40) total condylar prosthesis was the introduction of the duopatellar prosthesis. This design preserved the PCL and resembles the shape of a normal knee joint with the sagittal plane contour of the femoral component being anatomically shaped $(39,41)$. The early tibial prosthesis model 
was separated into medial and lateral parts, but a model was later developed that connected the bilateral parts. The patellar component was similar to that used in the Insall total condylar knee. The duopatellar prosthesis evolved into the kinematic prosthesis, which was widely used in the 1980s (9).

Influenced largely by the previous Freeman-Swanson design, the Insall et al. (40) total condylar design sacrificed both cruciate ligaments with sagittal plane stability maintained by the articular surface geometry. The femoral component, made of a cobaltchromium alloy, had symmetric condyles with a double curve and a flat patellar trochlear groove. The tibial prosthesis was made of polyethylene, had good conformity in flexion and extension, had anterior and posterior lips in the tibial joint surface, and had an eminence in the mid joint surface which provides anteroposterior and mediolateral stability. The tibial prosthesis also had a stem which allowed it to endure asymmetric loading. The patellar component was dome shaped and was composed of polyethylene with a fixation lug in the center which is fixed to the patella with bone cement. This original cemented total condylar prosthesis set the standard for survivorship of TKA (9).

While the total condylar prosthesis constituted the beginnings of modern TKA, the design was not without fault. It had a tendency to subluxate posteriorly in flexion if the flexion gap was larger than the extension gap. Moreover, the lack of femoral rollback by the implant severely diminished flexion if the PCL was not functioning. To correct these problems, the Insall-Burnstein PCL-substituting or posterior-stabilized (PS) design was developed in 1978 (42). A central cam mechanism was added to the articular surface geometry of the total condylar prosthesis; it interacted with a central post on the tibial articular surface and effectively reproduced femoral rollback allowing further flexion. In the Insall-Burnstein design, the post and cam were symmetric in nature (43). The post was positioned centrally on the tibial insert and the cam was located posteriorly on the femoral condyles. The Insall-Burnstein design engaged the cam on the post at approximately $60^{\circ}$ of flexion, driving femoral rollback of the condyles on the tibial insert in a symmetric manner with increasing flexion until edge loading occurred around $120^{\circ}$ of flexion. As the first PS design, this cam-post configuration was the basis for all PS implants to follow. Most current total knee designs are derivatives of the Insall-Burnstein $\mathrm{PS} / \mathrm{CR}$ and kinematic designs (9).

\section{Modern TKA Design}

Today, many different TKA designs exist to address patient-specific needs. Two major design types of TKA used today are CR implants, in which the ACL is resected but the PCL is preserved, and PS implants, in which both of the cruciate ligaments are sacrificed but the function of the PCL is mimicked by a post and cam design. Although debated and investigated for many years, the superiority of one design (PS or CR) over the other remains undetermined. In 2005, a Cochrane systematic review on this topic was published including eight clinical trials comparing clinical, functional, radiographical, and quality of life outcome measures in patients who had received PS or CR prostheses; meta-analysis did not reveal a clear difference between the two treatments. The authors 
found that the inconclusiveness of their initial review prevented them from giving clear advice on whether to retain and to sacrifice the PCL (44). In 2013, an updated version of the review was published and expanded to include 17 total randomized controlled trials, seven of which were included in the initial review. Again, the authors were unable to discern a difference between clinical, functional, and pain outcomes between the two implant designs and attributed the inconclusiveness to the variability of the methodological quality and the quality of reporting found in the trials (45).

When clear clinical evidence prompting resection of the PCL is absent, the selection of PS versus CR designs in TKA is largely based on the surgeon's personal philosophy regarding the matter, surgical training, and experience. Advocates of PCL retention have cited maintenance of normal knee kinematics, protection of the bonecement interfaces from shear stresses, increased proprioception, and enhanced inherent stability (46-51). Furthermore, supporters claim that there is greater passive knee range of motion and enhanced quadriceps muscle power via the PCL-controlled femoral rollback mechanism (52-54). On the other hand, proponents of PCL substitution argue that resection of the PCL makes ligament balancing easier and allows for a more conforming femorotibial articulation, which may reduce polyethylene wear (42, 55). Additionally, those surgeons state that a more controlled replication of the rollback mechanism can be created by modifications of the prosthetic design (56). Finally, sacrifice of the PCL is sometimes necessary to balance knees with severe varus/valgus or flexion contracture deformities; therefore, PS designs are integral in the treatment of patients with such deformities.

In studies comparing kinematic patterns of native knees to knees after TKA, the normal motion was usually not reproduced by the modern TKA designs. During in vivo fluoroscopic analysis of normal knees, CR TKA knees, and PS TKA knees, Dennis et al. (12) revealed a number of differences in the observed kinematic patterns. The femur of the normal knee contacts the tibia anterior to the midpoint in the sagittal plane in full extension and translates posteriorly during flexion. The femur of the PS TKA knee contacts the tibia anteriorly, slightly less than the normal knee, and rolls back posteriorly during flexion similar to the normal knees. The femurs of the CR TKA knees contact the tibia posteriorly in extension and then translate anteriorly during midflexion; this phenomenon, termed paradoxical motion, is kinematically opposite of the normal knees and may be a factor in premature polyethylene wear (12). In a multicenter analysis of subjects with PS TKA and CR TKA, Dennis et al. (57) reported data remarkably similar to the previous study, showing a lack of customary posterior femoral rollback in CR TKA, instead showing paradoxical anterior translation with flexion. Posterior femoral rollback, less than that in normal knees, was routinely observed in PS TKA knees and was attributed to engagement of the femoral component cam with the tibial post (57). In another multicenter analysis, Dennis et al. (58) studied the axial rotation patterns of patients with normal knees and patients with fixed and mobile bearing PS TKA and CR TKA. While over 33 implants designs were included, the magnitude of internal rotation was much less than the normal knees on average (58). Furthermore, they found that all TKA subgroups had at least $19 \%$ of patients displaying reverse axial rotational pattern during deep knee bend and at least $31 \%$ during gait. Whereas the pivot location in a 
normal knee is located medially, the effective pivot location observed by Dennis et al. (58) was variable, including on the medial side, the center, and the lateral side. Normal axial rotational patterns are essential for proper patellar tracking, reduction of patellofemoral shear forces, and maximization of knee flexion (58). Blaha et al. (59) posited that the anterior/posterior stability of the medial side of the normal knee is not usually present in a total knee when the knee is flexed. He proposed that a TKA designed to have a stable medial side combined with a lateral side that accommodates rotation of the tibia around the medial side through interaction of a spherical-shaped femoral component and a medially conforming tibial component may better replicate the kinematic patterns of a normal knee and confer better stability throughout gait (59).

Although CR or PS implants are generally regarded as a generic "type" of TKA, there are numerous designs available and each of these designs can vary considerably in the coronal and sagittal radii of their bearing surfaces and, in the case of PS implants, in the configuration of their cam-post mechanism. Variations in radii and cam-post mechanism are likely to impact the kinematic patterns and overall performance of the implant designs. Traditional TKA designs utilized multiple discreet radii to define the sagittal plane curvature of the femoral component; this geometry was meant to replicate the function of the natural knee joint by following the J-curve principle. A large primary distal radius was used as the principal load bearing surface during low flexion, high frequency activities, such as standing or gait, to reduce stress on the tibial insert. A smaller posterior radius of curvature reduced implant conformity and enabled the necessary knee rotation and rollback to facilitate deep knee flexion (60). In these traditional multi-radius designs, the distal radius instantaneously shifts to a smaller posterior radius at a specific flexion angle, typically between $15^{\circ}$ and $30^{\circ}$. However, this abrupt reduction in J-curve radii in midflexion caused abrupt changes in the center of rotation of the femur and abrupt reductions in tibiofemoral conformity, leading to a decrease in stability at midflexion (61). Using a deep knee bend simulator to assess the kinematics of multi-radius designs, Clary et al. (61) found that the sudden reduction in the radii of curvature of the femoral condyle was the underlying cause of the clinically "paradoxical" anterior femoral translation that is often observed in both PS and CR knees, although reportedly occurs more frequently in PCL-retaining knees $(62,63)$. Anterior motion of the femur on the tibia has the potential to adversely impact outcomes in several ways; anterior positioning of the femur reduces tibiofemoral range of motion, whereas relative tibiofemoral sliding increases the potential for insert wear while reducing quadriceps moment arms and efficiency (63-66) .

While it has long been held that flexion occurs about a constantly changing center of rotation, which is the basis for multi-radius designs, Hollister et al. (67) proposed instead that knee motion occurs about a single, fixed flexion/extension axis located in the posterior condyles. It was postulated that this single, fixed flexion/extension axis may be approximated by the transepicondylar axis (67). To ensure consistent tension in the collateral ligaments throughout the functional range of movement and to address the midflexion instability observed in multi-radius designs, the first "single radius" femoral component was introduced in 1996 based on this premise (Scorpio; Stryker, Mahwah, $\mathrm{NJ})$. Somewhat of a misnomer, the term single radius implies that a sudden radius 
transition does not exist, which is not the case. Instead, the transition from distal to posterior radii occurs earlier in the flexion cycle, typically around $10^{\circ}$ of flexion. Using fluoroscopic evaluation of in vivo kinematics of patients with single radius and multiradius CR implants, Kessler et al. (68) found that the single radius design is more stable in terms of varus/valgus laxity than the dual radius design in midflexion. When comparing the multi-radius and single radius PS TKA designs, Mahoney et al. (65) and Ostermeier et al.(69) found that the single radius design provided a more posterior flexion/extension axis which lengthened the extensor mechanism arms, decreasing the quadriceps muscle force while reducing the joint reaction force. In a study of 16 patients with single radius PS TKA, Shimizu et al. (70) reported that the single radius implants did not show any paradoxical anterior femoral movement until $70^{\circ}$ of flexion. The femoral component was kinematically stabilized in mid-flexion ranges and posterior femoral rollback occurred in deeper knee flexion with this design (70).

In addition to varying the sagittal shape of femoral components, the positioning and shape of the post-cam mechanism varies greatly among PS implant designs affecting the guided or constrained motion of the femur on the tibia. The post-cam mechanism was introduced to prevent posterior subluxation of the tibia and to increase posterior femoral translation in PCL-substituting TKA designs (42). Li et al. (71) evaluated the kinematics of PS TKA under simulated muscle loads and demonstrated that knee motion after postcam engagement was independent of muscle loads. This finding indicated that the postcam mechanism plays a critical role in knee kinematics. Consequently, it is important to understand how different variations in positioning or geometry of the post-cam design will affect knee motion. In a review of post and cam design variations, Mihalko et al. (72) summarized how the differences in positioning and shape effects the kinematics and wear of the PS designs. A centrally located post will interact with an anteriorly located cam sooner during the flexion arc and the cam will move up the post with further degrees of flexion, decreasing the jump height and increasing risk of posterior dislocation, a rare by severe complication (72-74). On the other hand, a centrally positioned post will interact with a more posteriorly positioned cam later in the flexion arc and the cam-post contact point will remain towards the base of the post even in deep flexion, thereby maximizing the jump height and minimizing risk of posterior dislocation $(72,74,75)$. If the post is moved or angled posteriorly, a posteriorly situated cam will engage the post sooner in the flexion arc and will position the femoral condylar contact points more posteriorly as well $(72,73,75)$. Cam and post geometry in the transverse plane also affects tibiofemoral kinematics. In contemporary PS prostheses, the post-cam design features can typically be categorized into flat-on-flat or curve-on-curve surfaces (76). A post with a rectangular cross-section will constrain rotation of the femur on the tibia in the transverse plane to an extent, affecting the internal/external rotational laxity. The restrictive nature of the rectangular shape will likely cause edge loading of the cam or even the condylar edges of the femoral component, thereby potentially increasing polyethylene wear $(72,76)$. A post with rounded geometry in the transverse plane may reduce internal/external rotational constraint decreasing the likelihood of burnishing and edge wear $(72-74,76)$.

The contemporary TKA tibial component can either be a fixed or mobile bearing design and the difference between these two designs is dictated by conformity. 
Conformity of an implant is defined as the difference between the corresponding femoral and tibial curvature in the sagittal or coronal plane (77). Conformity has a direct impact on the performance of a TKA, particularly with respect to tibiofemoral contact mechanics and kinematics. However, oftentimes, the implications of conformity on the contact mechanics and kinematics of the prosthetic components is conflicting. Fixed bearing TKA with a high conformity bearing surface provides low contact stress but restricts motion of the tibiofemoral articulation skewing the kinematic patterns and producing high torque at the bone-implant interface. Conversely, a low conformity fixed bearing surfaces allows for greater freedom of motion but increases contact stresses in the tibial insert which increases polyethylene wear (78-80). The kinematic conflict between low stress articulations and free rotation cannot be solved in a fixed bearing design because the modular tibial insert is locked within the tibial baseplate. As such, mobile bearing inserts were introduced with the aim to reduce polyethylene wear and component loosening $(81,82)$. The mobile bearing TKA design offers the advantage of allowing increased implant conformity and contact area without dramatically increasing stresses transmitted to the tibial insert or fixation interface (83). By increasing sagittal plane conformity in mobile bearing TKA, in vivo fluoroscopic analyses have demonstrated improved control of AP translation with reduced paradoxical anterior femoral translation, particularly during gait (57). In a study of axial rotation in fixed and mobile bearing knees, Dennis et al. (58) found that the average magnitudes of axial rotation in the two cohorts were similar; however, the site of rotation differed. Similarly, Komistek et al. (84) and D'Lima et al. (85) observed that the mobile bearing TKAs typically rotated in conjunction with the femoral component. Therefore, in TKA designs with a rotating platform, axial rotation typically occurs on the inferior surface of the tibial insert, whereas rotation obviously occurs on the superior surface in fixed bearing TKA. This likely plays a role in reduction of polyethylene wear after mobile bearing TKA because the shear forces on the superior aspect of the bearing are reduced.

Building upon the aforementioned principles factors, Walker et al. (86) incorporated various design features of TKA implants in an attempt to achieve normal knee motion characteristics. Two reference TKA models were included and consisted of a simple multi-radius condylar replacement in which the PCL is resected with partially conforming bearing surface and a posterior-stabilized simple multi-radius condylar design with a partially conforming bearing surface. As with the previous studies, the simple condylar replacement demonstrated lack of posterior femoral displacement and external rotation with flexion as well as the occurrence of the paradoxical anterior translation when a shear force was applied (86). This highlighted the fact that even when the PCL is absent, this paradoxical motion can still occur. The PS simple condylar design with partially conforming bearing surface was able to achieve posterior femoral displacement once the cam and post engaged at the set point of $90^{\circ}$ flexion (86). However, the PS design did demonstrate anterior displacement in the range of $30^{\circ}$ to $75^{\circ}$ of flexion. Moreover, both "reference" designs displayed average internal femoral rotation throughout the neutral path motions indicating that the posterior displacement of the medial side exceeded that of the lateral side, which was contrary to the kinematic patterns of the natural knees (86). 
The next two design variations Walker et al. (86) investigated were multi-radius femoral components that were to facilitate posterior femoral rollback through a patellar groove as opposed to a post and cam mechanism. It must be noted that these two designs would require resection of the PCL. In implant model S, the central ramp guides the posterior femoral displacement at flexion angles past $60^{\circ}$. Model $\mathrm{T}$ utilized the same femoral component as model $\mathrm{S}$ but included a tibial insert that is more dished medially, which induced a medial pivot that allowed for external rotation of the femur with flexion. In essence, model $\mathrm{T}$ included variations that would potentially allow for posterior femoral rollback and external rotation of the femur with flexion. The basic symmetric ramp model, model S, was able to produce posterior femoral displacement with flexion; however, when an anterior shear force was applied, the design did demonstrate a paradoxical anterior motion, albeit only $3 \mathrm{~mm}$ which was a vast improvement over the prior two models (86). In model T, the more medially dished tibial insert did facilitate progressive external rotation of the femur with flexion; however, this design also demonstrated $3 \mathrm{~mm}$ of paradoxical anterior displacement (86).

Finally, to address the observed paradoxical anterior displacement observed in the four previous designs, Walker et al. (86) applied the single radius principle to models U and $\mathrm{V}$. In model $\mathrm{U}$, the posterior radius was continued anteriorly to form a profile for medial and lateral recesses. The tibial surface from model $\mathrm{S}$ was altered to include medial and lateral anterior pads, that corresponded to the recesses of the femoral component where the larger anterior radius transitioned to the smaller posterior radius. Walker et al. (86) hypothesized that the femoral-tibial conformity in the sagittal plane would prevent anterior displacement up to $60^{\circ}$ of flexion; at which point, the central patellar groove, or ramp, can guide posterior femoral rollback. In model $\mathrm{V}$, the single radius principle was also applied; however, the medial side had a recess which the lateral side did not. Using the same medial pivot principle as the tibial insert for model $\mathrm{T}$, the tibial insert of model was less dished laterally and more dished medially with the addition of a medial pad that corresponded to the medial femoral recess. Walker et al. (86) hypothesized that this design would not only eliminate the paradoxical anterior femoral displacement, but also allow for additional rotational freedom in extension. As predicted, the anterior recesses of the single radius design of model $\mathrm{U}$ and $\mathrm{V}$ virtually eliminated the paradoxical motion. However, the provision of both medial and lateral recesses in model $U$ limited the freedom of internal/external rotation in extension to only a few degrees. By providing only a medial recess, model $\mathrm{V}$ was able to have anterior-posterior stability on the medial side, coupled with rotational freedom throughout the entire flexion cycle (86).

To date, no TKA design has been developed that perfectly reproduced normal knee motion. Currently, there are numerous TKA designs on the market. Each of the designs varies slightly in hopes that it will be able to recapture natural knee kinematics. The variety of TKA designs currently on the market suggests there is still not a consensus amongst designers and manufacturers on the effects of geometry on wear and kinematics (79). However, investigational studies, such as the one discussed above by Walker et al. (86), brings the orthopedic and engineering community one step closer to achieving that elusive goal. 


\section{Implant Materials}

While there are many variations of knee implants on the market today, a modern TKA prosthesis typically consists of a femoral component, a tibial component, a tibial insert, and a patellar component as shown in Figure 1-3. Femoral components on the distal end of the femur are typically made of cobalt-chromium-molybdenum (CoCrMo) alloy, but can also be fabricated with ceramic materials, such as alumina or zirconia. The tibial baseplate on the proximal end of the tibia is made of a metal alloy, either titaniumaluminum-vanadium (TiAlV) or CoCrMo. The metal byproducts released from the implants are mainly due to combined effects of mechanical wear and corrosion (87). The prostheses are fixed to the bone using polymethylmethacrylate (PMMA), also called bone cement. More recent designs forego cement fixation and are press-fit with a porous coating that is intended to induce biological fixation through osseointegration. The tibial insert, which sits atop of the tibial baseplate and articulates with the femoral component, and the patellar button, which is used to resurface the posterior side of the patella, are composed of ultra-high-molecular-weight polyethylene (UHMWPE). Tibial insert wear occurs from a combination of rolling, sliding, and rotational motions on the bearing surface, which may lead to delamination, pitting, and fatigue failure of the tibial insert surface. Polyethylene wear also has been observed on the undersurface, or backside, of modular tibial knee inserts $(88,89)$. Additionally, modes of sterilization, such as gamma radiation, used when manufacturing the polyethylene inserts have been shown to cause increased wear by introducing oxidation to the insert when placed in the body, causing delamination of the component surface as well as subsurface damage $(90,91)$.

\section{Surgical Factors Related to Implant Wear}

An estimated 20 or more surgical steps are completed during arthroplasty, and at least 5 or more variations of each step exist. Taking these estimations into account, over 3 million different variations in surgical technique can potentially occur in primary TKA. Successful outcomes following primary TKA and long-term implant durability are believed to be dependent on the aforementioned implant-specific factors as well as surgical factors. During the procedure, the surgeon must adhere to the following principles to ensure optimal outcomes: appropriate sizing of the individual components, alignment of the components to restore the chosen reference axis, recreation of equally balanced soft tissues and gaps in flexion and extension, and optimal patellofemoral tracking. While each of these factors were not specifically assessed in this study, their role in the etiology of failure were noted in this section.

\section{Proper Alignment of Components to Restore a Reference Axis}

Rotational positioning of femoral and tibial components is regarded as a critical aspect of TKA because malrotation of the components may lead to patellofemoral problems, flexion instability, excessive wear of UHMWPE insert or post, stiffness, and abnormal gait patterns (92). Proper alignment is believed to decrease both the mechanical 


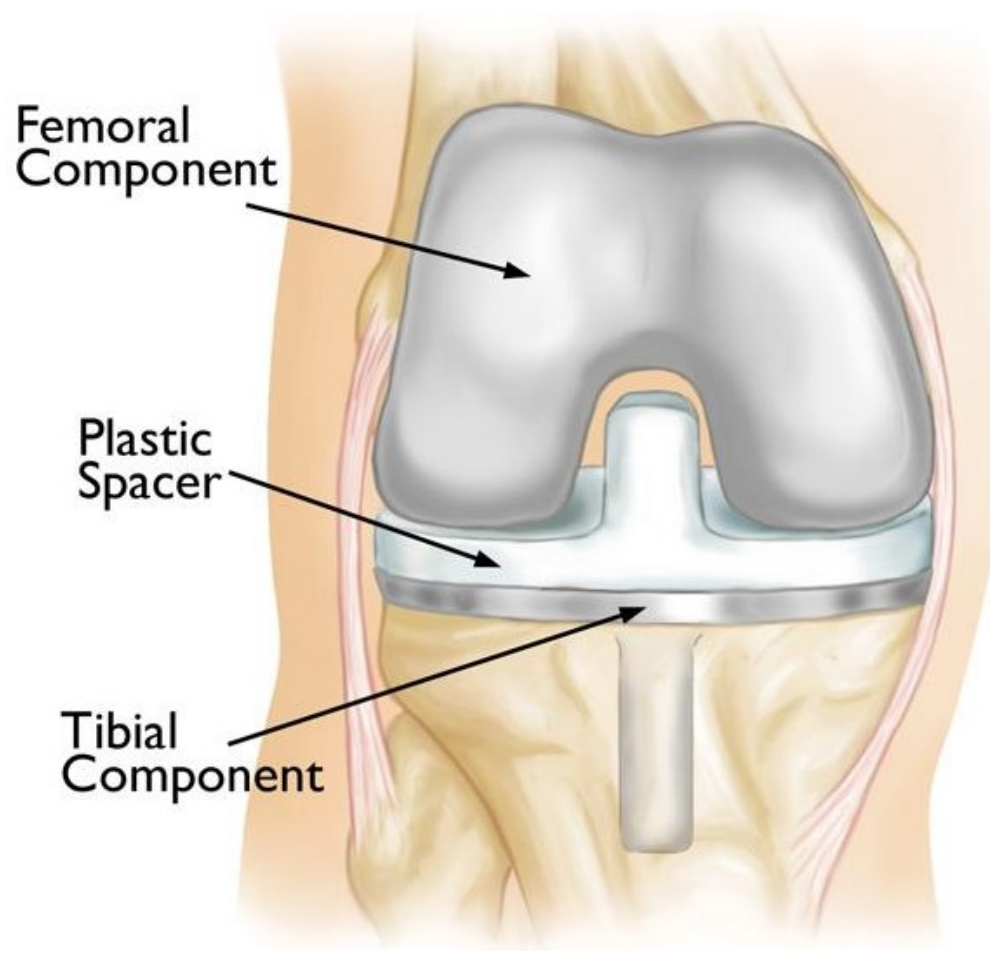

Figure 1-3. Total Knee Arthroplasty components

Reprinted with permission. Manner PW. Knee replacement implants. OrthoInfo. (C) American Academy of Orthopaedic Surgeons. http://orthoinfo.aaos.org. Accessed 18 August 2017. 
and shear stresses placed on the bearing surfaces, as well as the bone-implant interfaces (80). Various alignment axes and surgical techniques have been proposed to attain the goal of proper alignment of TKA components. There are three alignment philosophies currently suggested for use in TKA: anatomical, mechanical and kinematic alignment methods. Each of these philosophies use a different reference axis from which the distal femoral and proximal tibial cuts are based.

The anatomical axis of the lower extremity is defined in relation to the intramedullary canals of the femur and tibia. On antero-posterior evaluation, the anatomical and mechanical axes of the tibia coincide exactly, but, in the femur, the two axes make an angle of 5 to 7 degrees $\left(^{\circ}\right)(93)$. Originally described by Hungerford and Krackow (94), anatomic alignment sought to position the femoral and tibial components in such a way as to recreate the joint line. This optimal alignment, they believed, was 2 to $3^{\circ}$ varus in relation to the mechanical axis of the lower extremity (94). Per this reference axis, the proximal tibia was cut at $3^{\circ}$ varus to the anatomical axis of the tibia and the distal femur was cut at an angle that was the difference between the anatomic and mechanical axis of the femur, approximately 8 to $9^{\circ}$ valgus (94). When combined, the total alignment was approximately $6^{\circ}$ valgus that approaches the normal tibiofemoral angle and provided for a joint line that is parallel to the ground during normal gait (94).

The method of mechanical axis alignment in TKA was first described by John Insall (95). The mechanical axis of the lower extremity passes from the center of the femoral head to the center of the talar dome in the ankle (93). Insall et al. (95) posited that the mechanical axis approximates normal knee alignment and is easily identifiable. Furthermore, he suggested that the increased forces across the medial joint component in an anatomically aligned knee lead to medial tibial plateau fixation failure (95). Even though the joint loading forces between compartments are even during the stance phase of gait, joint forces will become uneven during the gait phase due to a laterally directed ground reaction force (96). As such, when using the mechanical axis alignment method, the surgeon first makes a distal femoral cut at a valgus angle of typically 5 to $7^{\circ}$ to the mechanical axis of the femur, generally removing the same amount of bone that is to be replaced by the femoral component. However, if a PS prosthesis is used, an additional distal femoral resection of 2 millimeters $(\mathrm{mm})$ can be performed to equal the increase in the flexion gap that occurs when the PCL is removed (97).

Because the anterior and posterior femoral cuts determine the rotation of the femoral component and the shape of the flexion gap, surgeons must take care during resection to maintain correct alignment of the femoral component in the transverse plane (9). With respect to femoral component alignment, excessive external rotation increases the flexion gap medially by facilitating tightening of the lateral ligaments by overstuffing the lateral flexion gap and loosening of the medial ligaments in flexion which can result in flexion instability $(98,99)$. In other words, increased external rotation of the femoral component increases internal rotational laxity and constrains external rotational laxity in flexion. Contrarily, internal rotation of the femoral component can cause lateral patellar tilt/subluxation, patellofemoral instability resulting in dislocation, pain, and patellar component failure (100). Many reference points, correlating to different 
anatomical landmarks, have been proposed and are used to determine femoral component rotation, including: the transepicondylar axis, the anteroposterior axis, the posterior femoral condyles, and the tibial cut first technique, also known as the "flexion gap balancing" technique. Regardless of the method used for rotational alignment, the thickness of the bone resected from the posterior aspect of the femoral condyles should equal the thickness of the posterior condyles of the femoral component. The surgeon completes the distal femoral preparation for a CR prosthesis by making anterior and posterior chamfer cuts for the implant. If a PS design is used instead, the bone is removed for the intercondylar box to accommodate the housing for the post and cam mechanism.

Studies have demonstrated that malalignment of the tibial component in the transverse plane is related to chronic pain. More specifically, increased internal rotation of the tibial component has been correlated with chronic pain $(101,102)$. Relative internal rotation of the tibial component effectively increases the $\mathrm{Q}$ angle and changes the force vector on the extensor mechanism. Barrack et al. (102) proposed that the abnormal stress on the patella due and the surrounding soft tissue may explain the peripatellar pain reported by patients following TKA. The "traditional" method for tibial component alignment involves using the tibial tubercle as a landmark and placing the center of the tibial component at the medial third of the tubercle. However, since the tubercle is rotated externally relative to the tibial plateau, this method has been shown to create a tendency for external rotation of the tibial component (103). Additional landmarks that have been proposed to determine proper tibial component alignment include: the "Akagi" line (104), the central third of the tibial tubercle (105), the postero-lateral tibial corner (106), and the anterior tibial contour (107). Once proper tibial component alignment is achieved, the proximal end of the tibia is cut perpendicular to its mechanical axis with a cutting block oriented by an intramedullary or extramedullary guide in both the anatomical and mechanical axes techniques. The amount of posterior slope depends on the implant system being used and the amount of tibial resection is dependent on which side of the joint is used for reference, i.e. the more or less arthritic side (9).

Using anatomical landmarks for the rotational alignment of the femoral and tibial components is a widely accepted method. However, determining the component positions separately can lead to rotational mismatch between the femoral and tibial components. Retrieval and biomechanical studies have reported that femoro-tibial rotational mismatches alter kinematics of the joint causing increased contact stress on the tibial insert and patellar button leading to accelerated UHMWPE wear as well as femoro-tibial subluxation and patellofemoral dysfunction $(102,108,109)$. To address this disadvantage of all anatomical landmark techniques, the range-of-motion (ROM) technique was introduced in which the rotational alignment of the tibial tray is determined through conformity to the femoral component when the knee is put through a series of full flexion-extension cycles (110). However a study conducted by Ikeuchi et al. (110) found that the ROM technique resulted in more internal rotation of the tibial component than when anatomical landmarks were used.

Historically, the two aforementioned alignment strategies have been used to replicate the mechanical axis of the lower extremity by positioning of the components. 
Recently, following the research by Hollister et al. (67), Howell et al. (111) proposed a technique, deemed the "kinematically aligned" TKA. Kinematic alignment in TKA is based on the 3 functional kinematic axes about which the knee rotates as described by Hollister et al. (67). The first axis is a transverse axis, defined by the transepicondylar axis of the femur, in which the tibia flexes and extends. The second axis is a transverse axis located anterior, proximal, and parallel to the first transverse axis which depicts the motion in which the patella flexes and extends in relation to the femur. The third axis, a longitudinal axis, is perpendicular to the 2 previous axes and dictated the dynamic movement of internal and external rotation of the tibia in relation to the femur. In kinematic alignment, the principles for placing the components are as follows: 1) coaligning the transverse axis of the best-fitting femoral component with the primary transverse axis in the femur about which the tibia flexes and extends; 2) removing osteophytes to restore ligament length, motion, and stability; and 3) placing the tibial component so that the longitudinal axis of the tibia is perpendicular to the transverse axis as described by the transepicondylar axis (112). This method strives to reproduce nearnormal knee function by aligning the articular surfaces of the femoral and tibial components to the normal or pre-arthritic joint lines of the knee $(111,113)$. To do this, the femoral component is placed in a slightly more valgus and internally rotated position, while the tibial component is placed in a slightly more varus and internally rotated position, compared with the placement of the implants in a mechanically aligned TKA $(112,113)$.

\section{Recreation of Soft Tissue Gaps in Flexion and Extension}

Soft tissue balancing, or obtaining proper ligament tension, during TKA is a paramount step to assuring longevity of the replacement because it is critical to maintaining the stability of the implant and joint post-operation. The typical surgical approach to properly balance a knee during TKA involves ensuring an equal gap distance of the medial and lateral compartments in both extension and flexion restoring the knee to proper alignment $(114,115)$. This is accomplished by releasing ligaments on the medial or lateral side of the knee, depending on which side is tighter. In a varus deformity, the medial soft tissues are tighter than the lateral side. This deforming is most commonly found in the OA knee and multiple techniques have been described for its correction. In both a PS and CR prosthesis, the MCL is typically released to balance tension of the ligaments and gap distance of the joint. In a valgus deformity, the lateral soft tissues are tighter than the medial side. This deformity is most common in patients with RA and inflammatory arthropathies. The three-layer anatomy of the lateral side of the knee joint makes its soft-tissue balancing more complex than those with varus deformity. Depending on the extent of the valgus deformity, surgeons may release the LCL, iliotibial band, popliteus tendon, or the tendon of the lateral head of the gastrocnemius (9).

Instead of ligament release, the pie-crusting technique has been introduced as a tool to correct varus or valgus deformities with a milder affect than ligament release (114). During balancing, the contracted side of the soft-tissue sleeve is assessed for tight 
structures and then the surgeon applies multiple stabs with a scalpel blade or large needle parallel to the joint line to effectively elongate the areas of the soft-tissue sleeve that are under undue tension. The advantage of pie-crusting, especially on the lateral soft-tissue sleeve, is that is leaves a supporting tether that prevents a larger gap opening on the lateral side of knee in flexion. Regardless of the balancing technique used, the surgeon must be sure to check the tissue and gap balancing in both extension and flexion as laxity of some structures changes with motion of the joint (9).

Suboptimal soft tissue and gap balancing may be implicated in the etiology of some of the most common indications for revision-loosening, excessive wear, instability, and malalignment (116-122). If the soft tissues are not balanced such that the lateral and medial joint gaps are equal throughout a range of flexion angles, it may lead to increased loading in one compartment such that the implant will not be able to function as intended. Excessive ligamentous laxity has been reported to increase anterior-posterior (AP) translation and internal-external (IE) rotation which leads to force concentrations that are outside of the intended design parameters of the TKA components and that exceed the material properties of the tibial insert facilitating increased UHMWPE wear $(78,123-126)$. There is a paucity of literature evaluating the relationship between joint laxity and metallic wear debris production following TKA. As such, optimal ligament laxity has yet to be clearly defined and is largely subjective, based on the surgeon's experience and intraoperative assessment.

\section{Proper Patellofemoral Tracking}

In addition to proper alignment of components and tissue balancing, the surgeon must ensure proper patellofemoral tracking. Patellar maltracking may result from component malpositioning and limb malalignment, prosthetic design, improper patellar preparation, or soft-tissue imbalance (127). As previously discussed, patellofemoral instability may result from internal malrotation of the femoral or tibial components. However, any alteration in knee kinematics and joint balance that increases the tightness of the lateral retinacular structures or increases the $\mathrm{Q}$ angle of the extensor mechanism to produce a laterally directed muscle vector may cause patellofemoral instability $(9,127)$.

If the patella is to be resurfaced, the prosthetic patella should be medialized to approximate the median eminence of the natural patella. Centralization of the prosthetic button requires the bony patella to track medially which forces it to function with a higher Q angle. Surgeons use the "no thumb" test of patellar tracking to determine adequate patellar stability. The reduced patella is observed within the femoral trochlea throughout the range of motion before retinacular closure. If the patellar button tracks congruently with minimal to no pressure applied to the lateral side of the patella, patellofemoral tracking is adequate and the surgeon can proceed with component implantation (9). If the patella does not properly track, a lateral retinacular release may be performed. Additionally, studies have shown that deflating the tourniquet prior to the "no thumb" test may attenuate the need for lateral release (128-130). As such, it is customary for surgeons to deflate the tourniquet prior to assessing patellar tracking. 


\section{Biological Responses to Wear Debris}

In general, modern materials for joint replacements are well tolerated by the body as long as they are in bulk form, are mechanically stable, and are not infected. However, normal usage of the joint following implantation results in the wear of the bulk material resulting in the production of debris. When wear products are generated, they may exist in one of two forms: insoluble, particulate debris and soluble, metallic ions. Particulate wear debris - polymers, ceramics, or metals - range in size from nanometers to millimeters whereas metal ions exist in soluble forms that may bind to serum proteins, remain in solution, or disseminate systemically (131). Polyethylene particles range in size between 0.23 to 1 micrometers $(\mu \mathrm{m})$, with an approximate mean size of $0.5 \mu \mathrm{m}(131)$. Metal particulate debris is generally an order of magnitude smaller with a mean size of $0.05 \mu \mathrm{m}$ (131). The production of these particles may incite adverse inflammatory reactions that are carried out by a variety of cells. However, the mechanisms of the biological response to the wear debris are complex and not well understood.

\section{Innate Immune Response to Wear Debris}

Debris from joint replacements, whether polymeric or metallic, stimulates the ingress of polymorphonuclear leukocytes (neutrophils, basophils, and eosinophils) and macrophages into the local site (132). Macrophages are believed to dominate the inflammatory events and recognition of wear particles by macrophages involves scavenger receptors that act as phagocytic receptors mediating direct non-opsonic phagocytosis of pathogenic microbes by macrophages (133). Depending on the size of the particulate debris, interaction with the macrophages may occur in one of three ways. Small particles, less than 150 nanometers $(\mathrm{nm})$ in size, are ingested by pinocytosis or endocytosis and do not cause cellular activation. Particles ranging in size from $150 \mathrm{~nm}$ to $10 \mu \mathrm{m}$ are phagocytosed. Larger particles, greater than $10 \mu \mathrm{m}$ in size cannot be phagocytosed and instead may interact with cells through pattern recognition receptors (PRRs), including nucleotide oligomerization domain-like receptors and toll-like receptors (134-137). PRRs are important in innate immunity as first-line defense for recognition of microbial patterns called pathogen-associated molecular patterns (133).

In response to stimulation via debris phagocytosis or interaction with PRRs, macrophages express chemokines, cytokines, proteolytic enzymes (matrix metalloproteinases, or MMPs), reactive oxygen and nitrogen species, prostaglandin $\mathrm{E}_{2}$ $\left(\mathrm{PGE}_{2}\right)$, and other substances of inflammation (138). Proinflammatory cytokines such as tumor necrosis factor (TNF)- $\alpha$, interleukin (IL)-1 $\beta$, and IL-6, among others, sequentially lead to increased production of RANKL (receptor activator of nuclear factor- $\kappa \mathrm{B}$ ligand) (135). The RANK/RANKL/osteoprotegerin (OPG) pathway directly regulates osteoclast activity and bone resorption. Overactivity of RANK/RANKL system and increased expression of these inflammatory molecules have been demonstrated in aseptic and septic loosened THAs, suggesting a role in prosthetic failure (139). Additionally, in adaptive immune responses, such as delayed type hypersensitivity (DTH), macrophages can 
activate T-cells through antigen presentation, which will be discussed further in the next section. While the mechanisms of the biological response to wear debris have yet to be delineated, this chronic inflammatory scenario ultimately leads to upregulation of pathways leading to bone destruction and downregulation of pathways leading to bone formation.

\section{Delayed-type Hypersensitivity}

The prevalence of metal sensitivity among the general population is approximately 10 to $15 \%$. Nickel is the most common metal sensitizer, with approximately $14 \%$ of the population demonstrating dermal reactivity, followed by Co and $\mathrm{Cr}(140)$. The prevalence of metal sensitivity among patients with well-functioning and poor functioning implants has been reported to be approximately $25 \%$ and $60 \%$, respectively, as measured by dermal patch testing (140). Delayed cell-mediated hypersensitivity may be implicated as a cause of tissue damage and loosening in instances of low wear. Due to their small size, metal ions can bind to serum proteins forming a hapten-protein complex $(140,141)$. The binding of metal ions alters the protein conformation so that immunogenic neo-epitopes are formed (87). These metal-protein complexes are considered to be candidate antigens for eliciting DTH responses (140). At the same time, macrophages can also phagocytose metal haptens, processing them along the endolysosomal route and finally presenting haptens on class II major histocompatibility complex (MHC-II) molecules.

It is also postulated that metal ions can also activate $\mathrm{T}$ cell receptors directly (87). Once the T-helper cells are antigen-activated, they secrete a variety of cytokines that recruit and activate macrophages, monocytes, neutrophils, and other inflammatory cells. Activated macrophages, because of their increased ability to present MHC-II and secrete certain cytokines, can trigger the activation of more $\mathrm{T}_{\mathrm{DTH}}$ cells, which in turn activate more macrophages; this self-perpetuating response can create extensive tissue damage (140). The specific T-cell subpopulation, the cellular mechanism of recognition and activation, and the antigenic metal-protein determinants elicited by these metals remain incompletely characterized. DTH reactions may cause the tissue damage and loosening observed in instances of low wear.

\section{Adverse Local Tissue Reactions}

Adverse local tissue reaction (ALTR) refers to untoward histological reactions (often associated with clinical manifestations) that occur when metal implants corrode $(142,143)$. Evidence of direct inflammatory cell-induced corrosion of non-modular femoral components has been previously reported (144-146), although it remains a controversial topic. When metallic particles are phagocytosed by macrophages and giant cells, the acidic intramedullary environment of the lysosomes facilitate corrosion of the CoCrMo particles, producing high intracellular levels of ions, which may cause cell death. In excess, metallic corrosion products may cause adverse local tissue responses or 
systemic toxicity. Furthermore, it has been demonstrated that metallic debris, in ionic form, decreases the proliferation of osteoblasts; as such, metal wear by-products may play a role in the etiology of osteolysis (147-150).

ALTR may present as metallosis, aseptic lymphocytic vasculitis-associated lesions (ALVAL), pseudotumors, or osteolysis. Metallosis, shown in Figure 1-4, is the macroscopic staining of the soft tissues and is associated with abnormal wear, such as micromotion at modular junctions or when the non-metal bearing fails facilitating metalon-metal articulation in TKA or THA $(151,152)$. ALVAL describes a periprosthetic inflammatory reaction that is similar to a type IV hypersensitivity response; it is characterized by soft tissue necrosis, lymphocytic infiltration including perivascular cuffing, and abnormal joint fluid (142). Pseudotumors are large cystic masses that may be cystic, solid, or a combination; they are usually, but not always, symptomatic, and histology tends to show much more extensive lymphocyte infiltration and tissue necrosis than ALVAL $(142,153)$. It has been suggested that these abnormal soft-tissue reactions may be attributed to two etiologies: wear-related cytotoxicity and hypersensitivity (154). Although ALTRs were originally believed to be associated with metal-on-metal THAs, clinicians and researchers continue to present case reports and studies in which patients with metal-on-polyethylene THA or TKA have metallosis $(151,152)$, pseudotumor formation (155), elevated tissue metal concentrations (140), and elevated systemic metal levels (156-158) highlighting the need for further research into the wear of metallic orthopaedic components.

\section{Systemic Effects and Associated Toxicity of Metallic Debris}

Because many of these metal ions are naturally occurring in the human body and are required for normal physiology, the normal human serum concentrations must first be established. Cobalt, whose reference value is 0.8 microgram per liter $(\mu \mathrm{g} / \mathrm{L})$ in serum, is an essential cofactor in vitamin B12 metabolism. Chromium, an essential trace element, is required for maintenance of proper carbohydrate and lipid metabolism and exists in valence states ranging from -2 to +6 , with hexavalent chromium, $\mathrm{Cr}^{6+}$, and trivalent chromium, $\mathrm{Cr}^{3+}$, being the most prevalent forms. While $\mathrm{Cr}^{3+}$ is the most prevalent form found in food and is generally regarded as nontoxic, $\mathrm{Cr}^{6+}$, a strong oxidizing agent, is classified by WHO as a class 1 human carcinogen (159). Normal serum level of $\mathrm{Cr}$ is approximately $0.15 \mu \mathrm{g} / \mathrm{L}$.

In recent past, excessive metal wear has been a concern for patients who have received metal-on-metal hip replacements because of the adverse immune reactions, such as hypersensitivity, metallosis, and pseudotumor formation, that have been reported (149, $160,161)$. As such, certain cutoff values have been established that may be indicative of excessive wear or ALTR. Serum Co concentrations exceeding $1 \mu \mathrm{g} / \mathrm{L}$ is typically associated with excessive wear in a poorly functioning prosthesis (131). Serum concentrations of $\mathrm{Cr}$ greater than $0.6 \mu \mathrm{g} / \mathrm{L}$ suggests significant prosthesis wear (162). Plummer et al. (163) reported average serum Co and Cr concentrations of $11.2 \mu \mathrm{g} / \mathrm{L}$ and $2.2 \mu \mathrm{g} / \mathrm{L}$ in patients with ALTR and trunnionosis at the modular head-neck junction, 


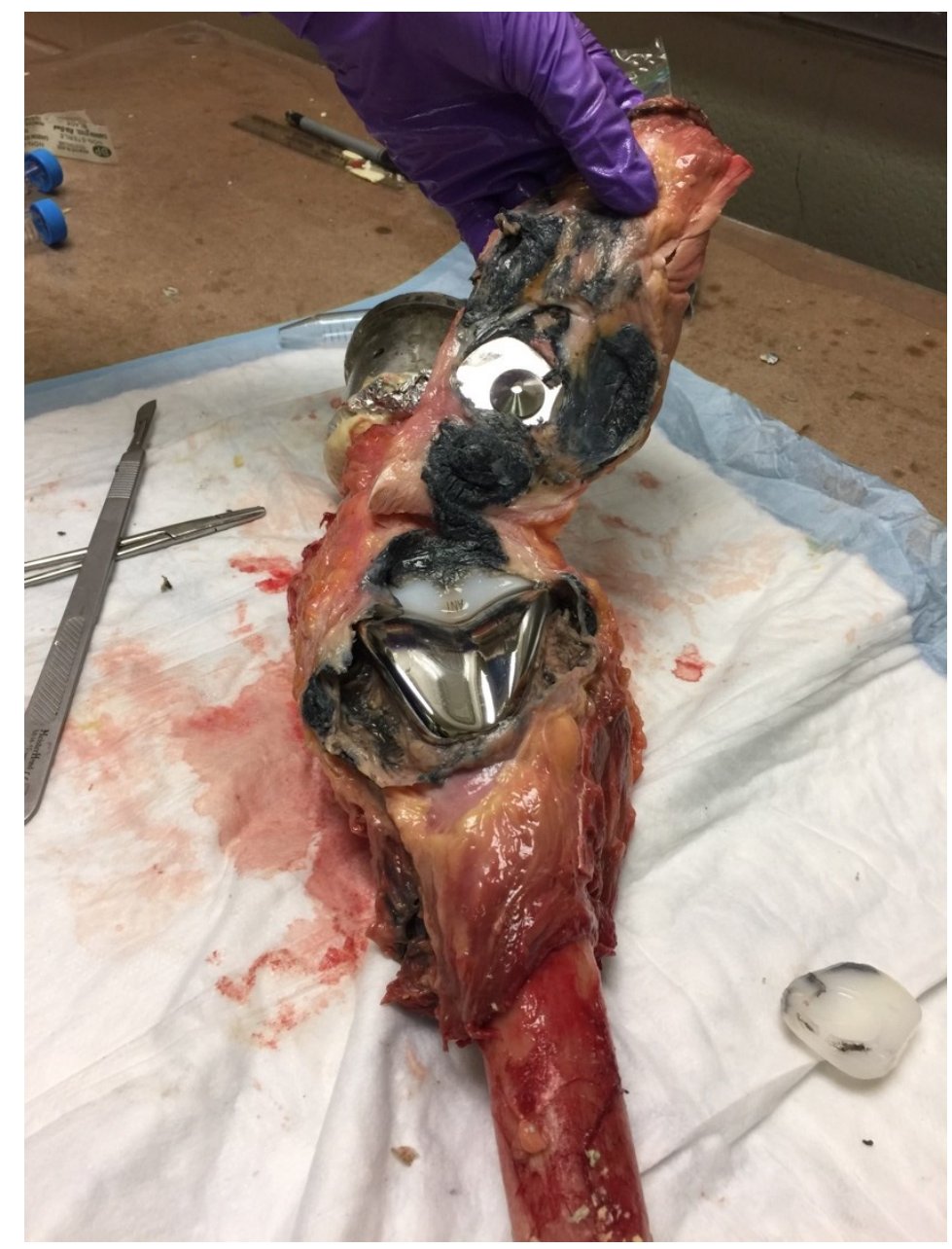

Figure 1-4. Specimen RLU0426161B-L, retrieved in this study, presented with metallosis 
whereas McGrory et al. (164) reported average serum $\mathrm{Co}$ and $\mathrm{Cr}$ concentrations as low as $5.4 \mu \mathrm{g} / \mathrm{L}$ and $0.75 \mu \mathrm{g} / \mathrm{L}$ in a similar cohort of patients. Large doses of Co can produce adverse clinical manifestations such as polycythemia, hypothyroidism, cardiomyopathy, and carcinogenesis (142). In excessive levels, chromium can lead to nephropathy, hypersensitivity, and carcinogenesis $(140,165)$.

Titanium alloys are commonly used in medical applications due to their osteoconductive and corrosion resistant properties. Normal human serum level of Ti is less than $4.1 \mu \mathrm{g} / \mathrm{L}$. While Ti is not an essential trace metal required for normal homeostasis like Co and $\mathrm{Cr}$, patients with Ti-base alloy implants have demonstrated elevated levels in joint pseudocapsules as well as elevated spleen and liver concentrations (131). Furthermore, titanium particles appear have been demonstrated to be more inflammatory than $\mathrm{Co}, \mathrm{Cr}$, UHMWPE, or alumina particles and induce pro-inflammatory cytokine production, include IL-6, TNF-a, and monocyte chemotactic protein (MCP) -1 (166), all of which are implicated in mediating osteolysis. The systemic release of metallic ions is a major concern and requires diligent monitoring, although acute and forthright toxicity is exceedingly rare.

In a prospective study, Lons et al. (156) sought to quantify metallic ion release after TKA. In their cohort, average preoperative $\mathrm{Co}, \mathrm{Cr}$, and $\mathrm{Ti}$ blood levels were 0.22 $\mu \mathrm{g} / \mathrm{L}, 0.45 \mu \mathrm{g} / \mathrm{L}$, and $2.94 \mu \mathrm{g} / \mathrm{L}$. At 1 year following the index procedure, average $\mathrm{Co}, \mathrm{Cr}$, and Ti blood levels increased significantly to $1.41 \mu \mathrm{g} / \mathrm{L}, 1.27 \mu \mathrm{g} / \mathrm{L}$, and $4.08 \mu \mathrm{g} / \mathrm{L}$, exceeding the normally occurring reference values. Additionally, a few clinical studies have reported increased systemic metal ion levels in TKA patients following loosening or malfunctioning of the implant $(156,167)$. These studies highlight the ability for clinically significant metal release in metal-on-polyethylene non-modular primary TKA. As such, the present study sought to further investigate the release of metallic debris in primary TKA.

\section{Inflammatory Cytokines as Biomarkers of Inflammation and Osteolysis}

Many studies have assessed the prognostic and diagnostic utility of various inflammatory cytokines as biomarkers in improving evaluation of suboptimal outcomes following total joint replacement. Prior to selecting which cytokines to test in this study, previous studies were consulted to establish that each of these cytokines had been demonstrated to induce an inflammatory or immune response when exposed to wear debris. The majority of research on potential biomarkers that was found in literature used patients with THA. Furthermore, the studies that were consulted used synovial-like interface membrane, surrounding periprosthetic tissue, or serum as their medium for cytokine analysis. It is difficult to conclude if elevated serum cytokine levels are result of events at the total joint replacement interface or if other inflammatory processes are contributing to the cytokine release (168). As such, this study sought to investigate the concentrations of various inflammatory cytokines present in synovial fluid in wellfunctioning cadaveric primary TKAs. 
Because TNF-a and IL-1 were the first cytokines shown to stimulate bone resorption in vitro, these inflammatory cytokines have been at the center of the search to identify a biomarker for damage in total joint replacements. TNF- $\alpha$ has a catabolic effect on bone, indirectly supporting the survival and differentiation of osteoclasts (169). IL-1 $\beta$ induces differentiation and proliferation of osteoclasts as well as the production of MMPs and $\mathrm{PGE}_{2}$ from fibroblasts and synovial cells (170). In vitro studies have also demonstrated that wear particles generated from TKA implants stimulate macrophages to produce IL-6, which is believed to have a regulatory role in maintaining the inflammatory response within the bone-implant interface as well as having an adverse effect on bone remodeling and mediating the effects of IL- $1 \beta$ and TNF- $\alpha$ during human osteoclast formation $(171,172)$. Consistent with these in vitro findings, several clinical studies have demonstrated increased production of these cytokines in periprosthetic tissues and fluids of loosened prostheses $(166,173,174)$.

Chemokines are an important subset of cytokines that produce chemotactic agents for the purpose of cell recruitment. The chemokines particular to implant aseptic loosening pathology include IL-8, MCP-1, and macrophage inflammatory protein (MIP$1 \alpha$ ). IL-8 has been demonstrated in vitro to be secreted by peri-implant cells such as macrophages and mesenchymal stem cells when exposed to different types of wear particles like Ti, CoCr, and polyethylene (175). Nakashima et al. (176) observed MCP-1 and MIP-1 $\alpha$ expression in all tissue samples from failed arthroplasties, establishing their presence in the inflammatory cascade of arthroplasty failure. Moreover, they were able to induce expression of MIP-1 $\alpha$ and MCP-1 by macrophages in cell culture after exposure to different types of wear particles (176). MCP-1 is potently chemotactic for monocytes but can also recruit macrophages, natural killer cells and T cells. A study by Dasa et al. (177) revealed elevated MCP-1 concentrations in revision samples of aseptically loosened TKAs when compared to OA and RA synovial fluid samples, demonstrating its potential as a biomarker for inflammation and osteolysis. MIP- $1 \alpha$ has a less clear role in implant debris-induced inflammation. Produced by a variety of peri-implant cell types, it is believed to likely be a central feature of adaptive immune responses via T-cells and Bcells to implant debris (178).

In addition to these major cytokines and chemokines associated with implant failure, granulocyte macrophage-colony stimulating factor (GM-CSF) and macrophagecolony stimulating factor (M-CSF) have both been suggested as mediators of the inflammatory cascade. GM-CSF is a hematopoietic growth factor with a regulatory effect on the transformation of immature macrophages into multinucleated giant cells that exhibit phenotypic and functional characteristics of osteoclasts (179). M-CSF is considered to be an essential mediator of osteoclast formation and release of M-CSF by activated cells in the peri-implant region is likely to be an important factor osteolysis (171). Recently, both GM-CSF and M-CSF have been implicated macrophage polarization. Macrophages exposed to lipopolysaccharide, IFN- $\gamma$, or GM-CSF assume a pro-inflammatory phenotype known as classical macrophage activation or M1 polarization. In contrast, macrophages exposed to IL-4, IL-13, or M-CSF assume an antiinflammatory phenotype known as alternative macrophage activation or M2 polarization (180). M1 macrophages are effective in antigen presentation and killing of phagocytosed 
pathogens and also produce inflammatory cytokines and chemokines, whereas M2 macrophages produce anti-inflammatory cytokines and instead participate in tissue regeneration and angiogenesis by secreting various growth factors and extracellular matrix precursors (181).

While the aforementioned inflammatory cytokines are involved in the innate immune response, several cytokines, including IFN- $\gamma$ and IL-2, mediate the adaptive immune response via lymphocytes. The type of lymphocytes that dominate the implant debris-associated responses are T helper cells, which are divided into two subtypes: Th1 cells that are critical in macrophage activation and Th2 cells that are engaged in B cell activation and humoral immunity. Th1 cells, characterized by production of IFN- $\gamma$ and IL-2, have been implicated as mediating metal DTH responses. While it has been difficult to readily identify these responses in peri-implant tissues by such signature cytokines, some studies using mRNA detection instead of tissue immunohistochemistry have shown the increased expression of these Th1 cytokines $(182,183)$.

\section{Objectives, Hypotheses, and Rationale}

As total knee arthroplasty continues to be one of the most commonly performed orthopaedic procedures performed annually, revision arthroplasties will continue to increase as well. The understanding of the etiology of aseptic implant loosening is still in its infancy and implant retrieval studies are of utmost importance to expanding this understanding. Of the implant retrieval programs in existence, most analyze failed implants retrieved at time of revision surgery. In choosing to solely analyze wellfunctioning implants retrieved at time of necropsy, the first objective of this study was to provide a baseline measurement of certain implant, surgical, and biological factors - such as polyethylene wear scores, femoral component damage scores, soft tissue laxity profiles, periprosthetic tissue metal concentrations, and inflammatory cytokine concentration in synovial fluid - that dictate the longevity and success of a TKA. The second objective was to compare the laxity profiles, periprosthetic tissue metal concentrations, and synovial fluid cytokine concentrations to determine if a correlation existed between each factor. Additionally, the tibial insert wear scores and the femoral component damage scores will be presented but not analyzed for comparison due to their subjective nature. The final and future objective of this study is to utilize these "baseline measurements" for comparison against failed implants retrieved at time of revision to elucidate if any meaningful relationships emerge. To this end, the following hypotheses were tested:

(a) Elevated tissue metal concentrations will be associated with decreased joint laxity. In other words, an inverse correlation will exist between periprosthetic tissue metal concentrations and joint laxity profiles. A more constrained laxity profile will cause increased contact pressures between the articulating surfaces facilitating abrasive and adhesive wear via third body particles and the subsequent release of $\mathrm{Co}$ and $\mathrm{Cr}$ particles or ions. Additionally, higher loads in the transverse plane will cause backside wear of the UHMWPE tibial insert against the TiAlV 
alloy or CoCrMo alloy baseplate inducing the release of $\mathrm{Ti}, \mathrm{Co}$, or $\mathrm{Cr}$ particles or ions.

(b) Elevated synovial fluid inflammatory cytokine concentrations will be associated with increased joint laxity. In other words, a positive correlation will exist between synovial fluid inflammatory cytokine concentrations and joint laxity profiles. A laxer joint profile has been demonstrated to increase UHMWPE wear of the tibial insert. Increased UHMWPE debris production will incite an inflammatory response mediated by the innate immune system. As such, cytokines specifically tied to the innate immune system will be elevated. It must be noted that an increase in inflammatory cytokines may also be caused by metallic debris.

(c) Elevated inflammatory cytokines in the synovial fluid will be associated with elevated periprosthetic tissue metal concentrations. In other words, a positive correlation will exist between tissue metal concentrations and inflammatory cytokine concentrations in the synovial fluid. Increased metal debris production will incite an inflammatory response that may be innate, adaptive, or a combination of the two in nature. Regardless of the type of immune response, macrophages are activated when challenged with wear debris and release inflammatory cytokines in response. It must be noted that an increase in inflammatory cytokines may also be caused by UHMWPE debris. 


\section{CHAPTER 2. METHODOLOGY}

\section{Experimental Overview}

The purpose of this study was to determine whether a correlation exists between laxity, inflammatory cytokines, tissue metal ion content, and wear scoring in wellfunctioning necropsy specimens to establish a baseline role of each of these factors in implant survivorship. Due to the many facets of this investigation, this section will provide a brief overview outlining the processes involved in the data collection and processing. Total knee arthroplasty necropsy specimens were retrieved from Medical Education and Research Institute (Memphis, TN) and RestoreLife USA (Elizabethton, $\mathrm{TN})$ with prior institution review board approval.

Dissection and retrieval were performed by a fellowship-trained, board-certified orthopaedic surgeon, Dr. William Mihalko. All superfluous skin and muscles tissue was excised from the area surrounding the joint capsule while carefully preserving the ligaments and other structures that contribute to stability of the joint. The joint center was identified and transverse cuts were made $180 \mathrm{~mm}$ superior and inferior to joint center along femur and tibia using a sagittal saw (Stryker Corporation, Kalamazoo, MI). The fibula was also transected $100 \mathrm{~mm}$ from the joint center $(114,184-189)$.

After retrieval, the specimens were labeled and placed in biohazard bags to be stored in the freezer until further testing. Thirty-six hours prior to testing, the specimens were removed from the freezer and thawed in a refrigerator. Before testing, fluoroscopy images (OrthoScan HD Model 1000-0001, OrthoScan, Scottsdale, AZ) were taken to assess if radiographic signs of osteolysis were present and all replacements were determined to be well-fixed per the images. Synovial fluid was aspirated from the joint using a syringe, centrifuged at 1,600 revolutions per minute (rpm) for 20 minutes to remove cell particulates, aliquoted into 1.5 milliliters $(\mathrm{mL})$ micro-centrifuge tubes, and stored in a $-80^{\circ} \mathrm{C}$ freezer.

Following synovial fluid collection, laxity testing was performed on a custom knee testing platform which has been previously studied and reported in published journal articles and theses $(114,184-193)$. Each specimen was mounted into the testing platform and the IE rotation, varus-valgus (VV) deflection, and AP translation was tested at $0^{\circ}, 30^{\circ}, 60^{\circ}$, and $90^{\circ}$ of flexion. At each flexion angle, the specimen was subjected to a 1.5 Newton-meter (Nm) IE rotational torque, $10 \mathrm{Nm} \mathrm{VV}$ torque, and a 35 Newton (N) AP directed force, and the corresponding deflection data was recorded using a custom LabVIEW program (National Instruments, Austin, TX) that had been previously detailed and validated (184).

After collection of the laxity data, a medial parapatellar incision was made to gain

access to the TKA components. Tissue samples were collected from the supra- and infrapatellar regions, the medial and lateral gutter, and from the tibia as depicted in Figure 2-1. Tissue was placed into collection tubes and stored at $-80^{\circ} \mathrm{C}$. The tissue-implant 


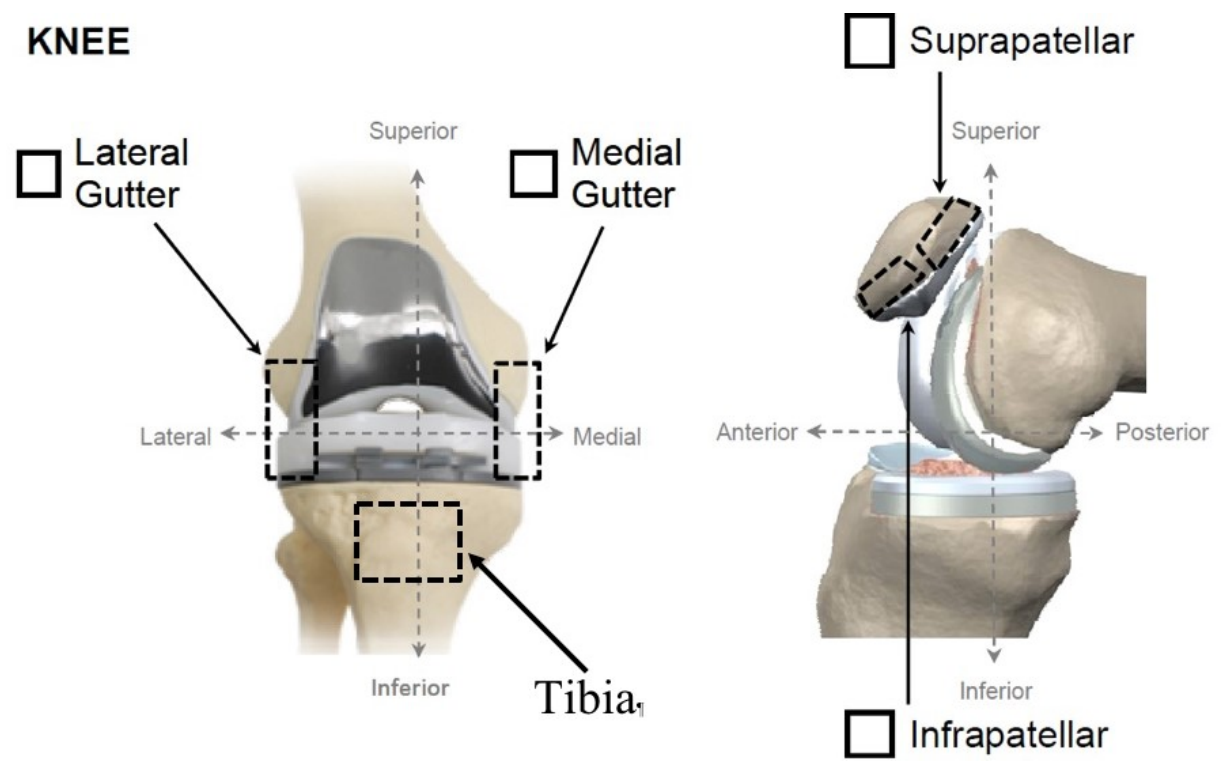

Figure 2-1. Periprosthetic tissue collection locations 
interface was assessed noting any abnormal conditions at time of retrieval. The implant was then removed from the bone using a chisel and hammer as recommended by ASTM Standard F561-13 (194), placed into biohazard bags, and shipped to Drexel University (Philadelphia, PA) for cleaning and wear scoring.

At Drexel University, the implants were cleaned per ASTM Standard F561-13 (194). Polyethylene inserts were analyzed for wear using the protocol proposed by Hood et al. (195) and the femoral condyle damage was assessed as previously described by Arnholt et al (196).

A bead-based multiplex assay using the Luminex MAGPIX platform (R\&D Systems, Minneapolis, MN) was performed on the synovial aspirates for simultaneous detection of human TNF- $\alpha$, IL-1 $\beta$, IL-6, IL-8, MCP-1, MIP-1 $\alpha$, MIP-3 $\alpha$, GM-CSF, MCSF, IFN- $\gamma$, and IL-2. Inductively coupled plasma mass spectrometry (ICP-MS) was performed at Brooks Applied Labs (Bothel, WA) on the periprosthetic tissue samples for determination of tissue metal concentrations. Finally, statistical analysis was conducted using SigmaPlot (Systat Software, Chicago, IL) to elucidate whether any correlations existed between the aforementioned factors.

The steps in this protocol were summarized in Figure 2-2 for each cadaveric TKA specimen. Procedures 2, 4, 5, 7, 8, and 9 will be explained in greater detail in the following sections.

\section{Fluoroscopic Analysis}

Prior to laxity testing and implant retrieval, fluoroscopically-assisted radiographs of each specimen were taken to assess if radiolucent signs of osteolysis were present. Since very small degrees of obliquity can obscure radiolucent lines adjacent to prostheses, fluoroscopy is useful for obtaining radiographs with clear visualization of the bone-prosthesis interface (197-200). An OrthoScan HD (Model 1000-0001, OrthoScan, Scottsdale, AZ) was utilized and standard AP and lateral views of each specimen were taken. Fluoroscopic radiographs of each specimen are included in the Appendix A (Figures A-1 through A-33). Fixation of the tibial component was examined in the AP and lateral planes. The beam was centered on the knee and rotation of the knee was set so that the pegs of the prosthetic component were symmetrical. To best visualize the fixation of the tibial bone-implant interface, vertical height of the tibial component was minimized such that the surface appeared two-dimensional, thereby ensuring that the beam was parallel to the interface. Fixation of the femoral component is examined in the lateral plane only. When assessing the fixation of the femoral component, the anterior and posterior bone-implant interfaces as well as the distal interfaces were examined. Again, the knee was aligned in the lateral plane such that the component was superimposed creating a two-dimensional view. All specimens were determined to be well-fixed per the absence of radiolucencies using the previously described and validated fluoroscopically-assisted radiographic technique (197-200). 


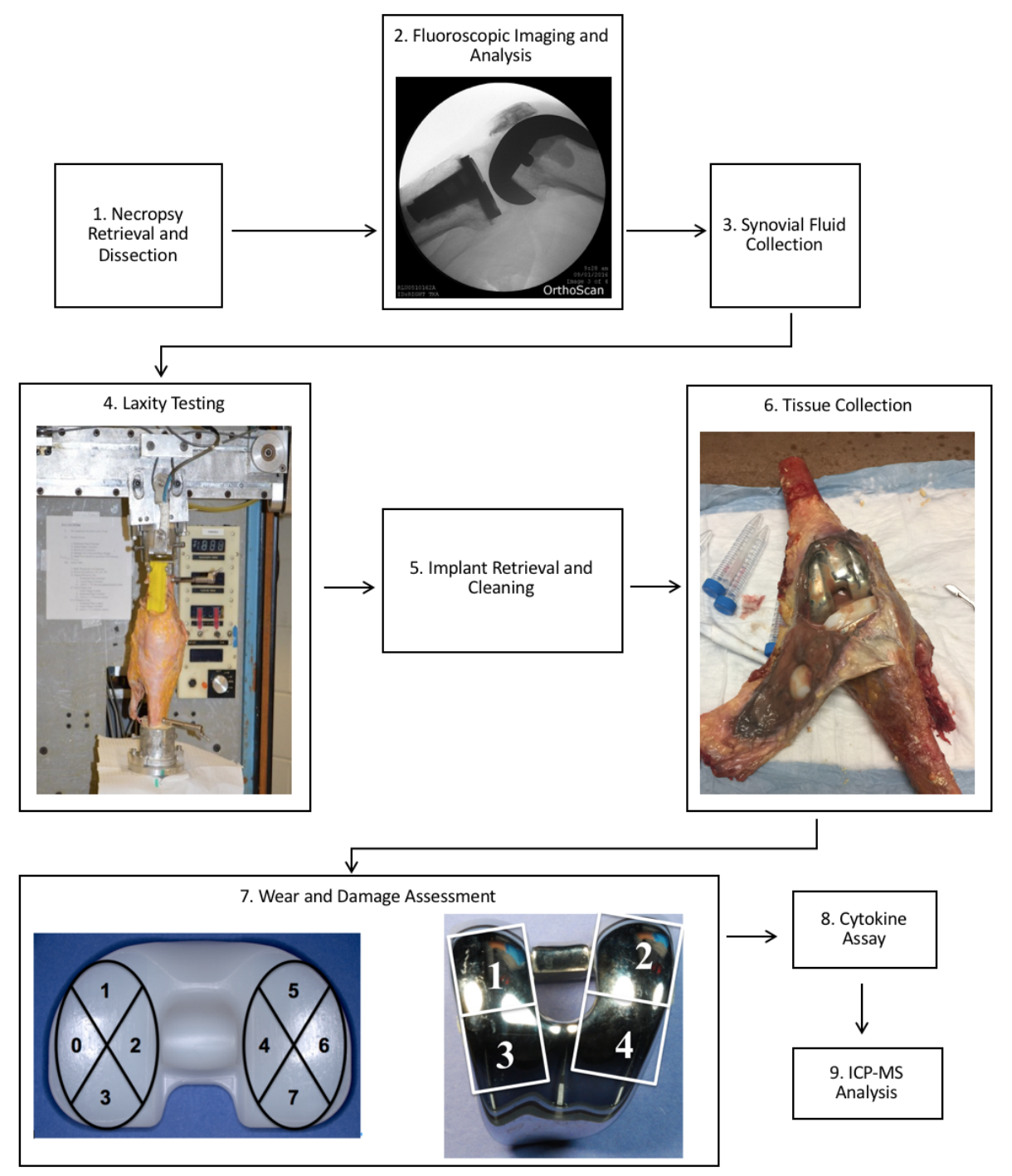

Figure 2-2. Summary of retrieval analysis process 


\section{Laxity Testing}

Laxity testing was performed on a custom knee testing platform, designed and manufactured in 1991 by Paul McLeod, shown in Figure 2-3. This testing apparatus was acquired by Dr. John William's laboratory at the University of Memphis in 2009 where it was restored to full working condition by 2010 through major efforts from Dr. Williams' graduate students: John Connor (190), Devin Conner (192), David Knox (191), and Jason Lindsey (193). Dr. Williams and the aforementioned students replaced bearings, transducers, and electronics, converted data acquisition platform from the original 1980s software to modern data acquisition software, and developed new programs for the kinematic analysis. It was then transferred to Dr. William Mihalko's laboratory at the University of Tennessee Health Science Center in April 2013. In 2014, Erik Woodard used the platform in his thesis, and, building upon Dr. Williams' students previous work, Erik further developed the programs that processed the data (184).

One of the functions of the knee testing platform is to measure the laxity or, conversely, the stiffness of the knee at various flexion angles. For this purpose, the knee was held at a fixed flexion angle and a pre-determined torque or force was applied at the tibial end while the resulting rotation or displacement was recorded. Cable connections on the knee testing machine provided data outputs from five force transducers and five angle potentiometers. The signals from the transducers and potentiometers travelled to their specific boards and were amplified before travelling through an analog to digital converter and an input/output board to a 12-bit data acquisition device which digitized the signals and displayed them in the custom LabVIEW program. The LabVIEW files were converted to Excel files and then run through a previously developed Matlab (Mathworks Inc, Natick, MA) program to extract data of interest and perform interpolations to identify points of interest in the data from the files (184).

Prior to testing, the femur and tibia were transversely cut approximately $180 \mathrm{~mm}$ from the joint center. The femur and tibia were centered using six pointed screws in metal pots coated with petroleum jelly and aluminum foil, for ease of removal. Once the femur and tibia were secured in place in their respective pots, urethane epoxy (Goldenwest MFG Inc, Cedar Ridge, CA) was poured into the pot and allowed to set for 30 minutes until completely hardened. The specimens were mounted in the machine with the tibia secured vertically and the femoral coupling locked in a neutral position as defined by the tibial position. Neutral rotation was defined as placing the femoral epicondylar axis perpendicular to the centerline of the machine. Because some specimens demonstrated flexion contracture, full extension was not always obtained and was instead defined as the minimum flexion angle of the knee specimen.

Specimens were tested throughout $0^{\circ}, 30^{\circ}, 60^{\circ}$, and $90^{\circ}$ flexion. An applied upward body weight force of $30 \mathrm{~N}$ was placed under the tibia to maintain joint contact during all tests. Each test was performed 3 times to pre-condition the specimen, and data was recorded on the fourth iteration. At each flexion angle, a $1.5 \mathrm{Nm}$ IE rotational torque was applied about the tibial axis while the femur was fixed and the tibia free to rotate 


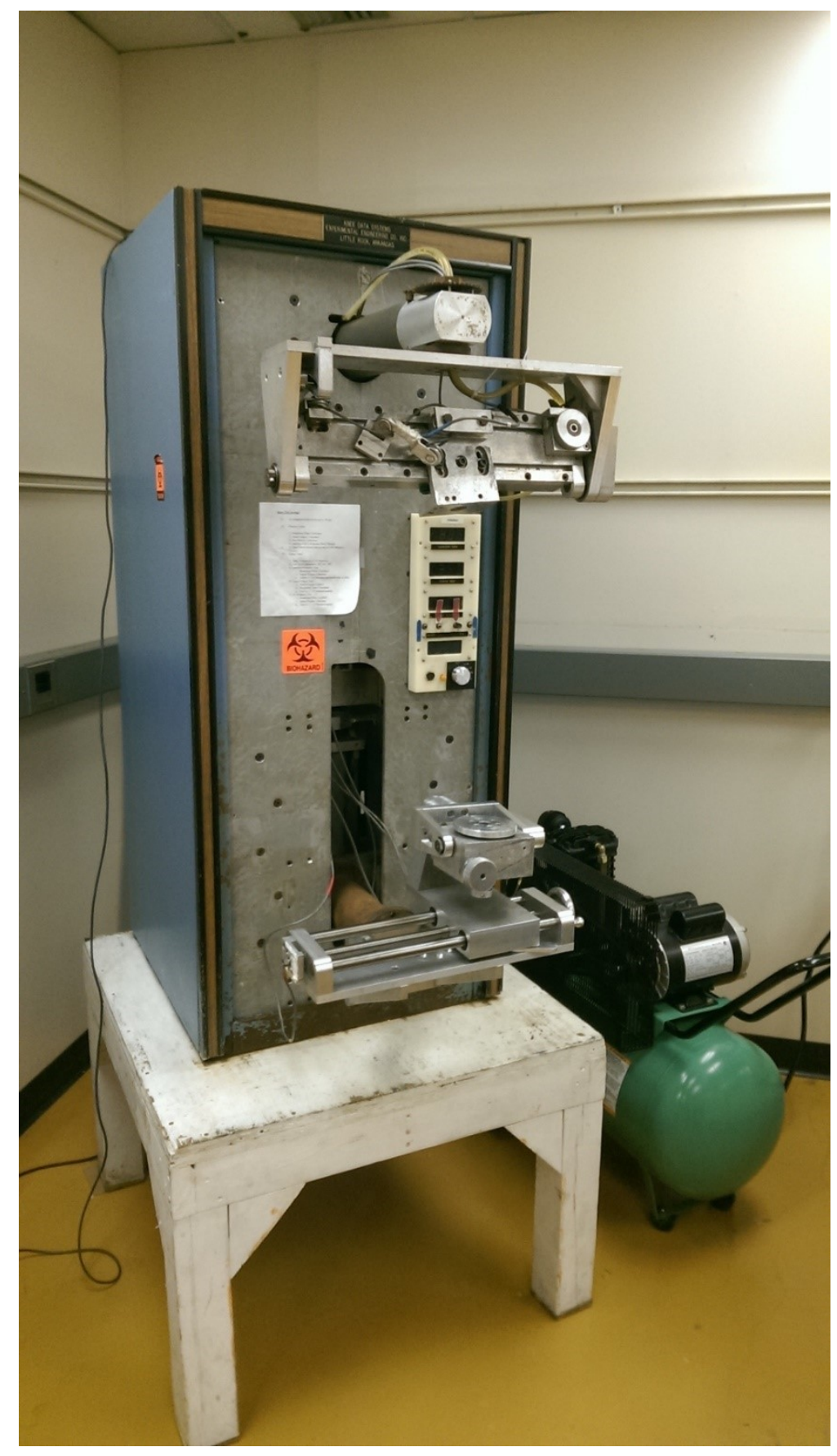

Figure 2-3. Custom knee testing platform in Dr. William Mihalko's lab 
about the joint center in the coronal plane. The deflection in degrees at $\pm 1.5 \mathrm{Nm} \mathrm{IE}$ torque in the transverse plane referenced from the normal neutral position was recorded to determine rotational laxity. A $10 \mathrm{Nm} \mathrm{VV}$ torque was also applied to each specimen at the distal end of the tibia, while internal-external rotation was unconstrained. The angular deflection in degrees at $\pm 10 \mathrm{Nm}$ of $\mathrm{VV}$ torque in the coronal plane referenced from normal neutral position was recorded to determine the valgus laxity for each test. Lastly, a device, which attached to the tibia, was used to apply a $35 \mathrm{~N}$ AP shear force perpendicular to the tibial axis. Both coronal motion and axial rotation of the tibia were unconstrained during this test. The displacement of the tibia relative to the femur at \pm 35 $\mathrm{N}$ was recorded to determine the AP laxity of each specimen. For all tests, sign conventions followed the Grood and Suntay coordinate system which specifies anterior displacement of the tibia in relation to the femur as positive, posterior displacement as negative, internal rotation as negative, external rotation as positive, varus motion as negative, and valgus motion as positive (14). While the tibia and femur each have a separate coordinate system, it is only necessary to define tibial motion in its own coordinate system since the femur is fixed throughout each test.

\section{Implant Cleaning}

The retrieved implants were cleaned using a method designed by Drexel University in accordance with ASTM Standard F561-13 $(194,196)$. Upon receipt of the shipment, implants were removed from biohazard packaging, examined to ensure all parts were present, and photographed for inventory with implant number visible. Each component was rinsed in cold water in a biohazard sink to wash away loose tissue. A 1:10 ratio of Discide detergent (AliMed, Dedham, MA) to water solution was prepared in a mixing cup and implants were placed in the solution for a 20 minutes soak. Implants were removed from the solution and brushes were then used to carefully remove remaining tissue from implant ensuring that the implant surface was not damaged or scratched. Next, the components were placed in a 1:10 ratio of bleach to water solution for a 20 minutes bath after which brushes were again used to remove loose debris. Following the bleach bath, the implant components were no longer considered a biohazard and were placed in a clean mixing cup. For the polyethylene components, the cleaning process ended at this stage. The metallic femoral and tibial components were then placed in a clean mixing cup filled with water. The cups were then placed in the ultrasonicator for 25 minutes. After the time period, the water was drained and refilled and the cups were replaced in the ultrasonicator for an additional 25 minutes water to remove any loose debris. The implants were then laid out on Versidry sheets (Thermo Fisher Scientific Inc, Rochester, NY) overnight to dry within the fume hood. The components were then packaged into separate bags to avoid scratching and labeled to await visual inspection for wear and damage scoring. 


\section{Wear Scoring}

After the cleaning process, wear was semi-quantitatively assessed by inspecting the articulating surfaces using a modified method of that proposed by Hood et al in 1983 (195). The superior side of the tibial insert, which articulates with the femoral condyles, was divided into 8 zones with 4 quadrants on each condyle as shown in Figure 2-4. The inferior side of the tibial insert, which is in direct contact with the tibial tray and should be fixed unless it is a mobile bearing design, was divided into 8 zones similar to the divisions shown in Figure 2-3. For PS implants, the stabilizing post was divided into anterior, posterior, medial, and lateral zones. Each zone was inspected using a light microscope with 10x magnification, when necessary, for 7 modes of damage: burnishing, pitting, delamination, abrasion, embedded debris, scratching, and surface deformation. Burnishing refers to highly polished areas on the articulating surface whereas pitting describes irregularly shaped depressions in the articulating surface measuring approximately 2 to $3 \mathrm{~mm}$ across and 1 to $2 \mathrm{~mm}$ in depth. Delamination wear is the removal of large sheets of polyethylene from the surface and abrasion is large areas with a shredded or tufted appearance often caused by contact with bone or cement. Embedded debris damage describes areas containing metal or PMMA debris deposited into the polyethylene surface. Scratching damage consists of areas containing indented lines generally found in the anteroposterior direction. Lastly, surface deformation refers to any permanent deformation of the surface of the insert and is typically caused by cold flow or creep of the polyethylene. Each section was graded for each wear mode and given a score of 0 to 3 based on the levels of damage present. A score of 0 was used to indicate that the damage mode was not present on that section of the insert, while 1 denoted damage covering less than $10 \%$ of the section, 2 denoted damage covering $10-50 \%$, and 3 indicated damage was present on over $50 \%$ of the section, as summarized in Table 2-1. Two independent observers inspected each implant and any significant discrepancy between observers were resolved in a meeting among the investigators. The two total scores were averaged for a final total score. The condylar scores were then summed to find the total condylar wear score, with a maximum possible score of 168 . The backside scores were summed to find the total backside wear score, with a maximum possible score of 168. When applicable, the post wear scores were summed to find the total post wear score, with a maximum possible score of 84 .

Similarly, the bearing surface of the CoCr femoral component was divided into 4 quadrants; zones 1 and 2 denote medial and lateral posterior surfaces whereas zones 3 and 4 denote medial and lateral anterior surfaces, as shown in Figure 2-5. Each section was assessed for three damage modes: scratching, indentations or lacerations, and pitting. As summarized in Table 2-2, a score of 1 described damage that covered less than 10\% of the section, a score of 2 indicated damage present over $10-30 \%$ of the surface, a score of 3 meant damage covering $30-50 \%$ of the section, and a score of 4 was given when damage covered more than $50 \%$ of the surface. The maximum possible damage score was a score of 16. 


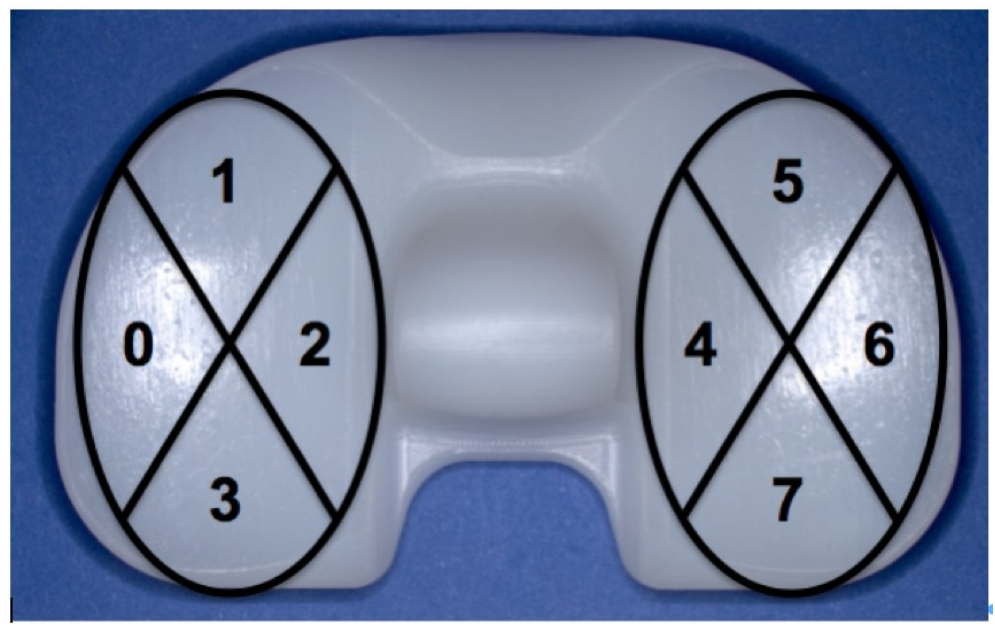

Figure 2-4. Technique used to divide the bearing and backside surface of tibial insert into 8 sections for wear scoring

Table 2-1. Summary of the polyethylene wear score quantification as proposed by Hood et al.

\begin{tabular}{cl}
\hline Score & \multicolumn{1}{c}{ Criteria } \\
\hline 0 & Wear mode absent \\
1 & Wear mode covers less than $10 \%$ of regional area \\
2 & Wear mode covers $10-50 \%$ of regional area \\
3 & Wear mode covers greater than $50 \%$ of regional area \\
\hline
\end{tabular}

Source: Hood RW, Wright TM, Burstein AH. Retrieval analysis of total knee prostheses: a method and its application to 48 total condylar prostheses. Journal of Biomedical Materials Research Part A. 1983;17(5):829-42. 


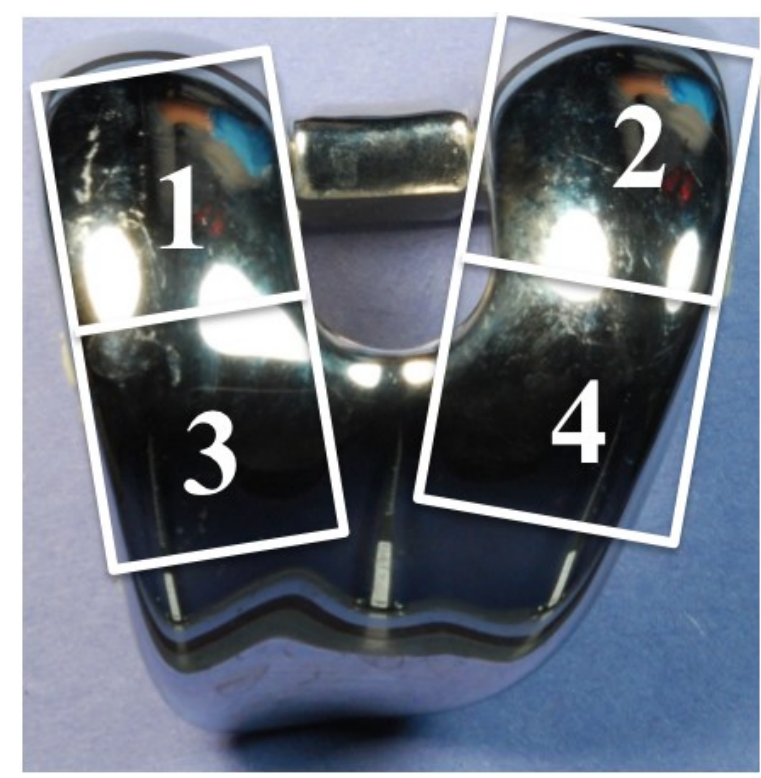

Figure 2-5. Scoring method for the femoral component.

Note: The femoral component bearing surface was split into posterior (1 and 2) and anterior (3 and 4) regions for damage scoring

Table 2-2. Criteria for femoral component damage scoring

\begin{tabular}{lcl}
\hline \multicolumn{1}{c}{ Damage } & Score & \multicolumn{1}{c}{ Criteria } \\
\hline Minimal & 1 & $\begin{array}{l}\text { 0 to 1 surface scratches with minimal coverage and } \\
\text { no apparent corrosion } \\
\text { Penetrating scratches on }>10 \% \text { surface, very faint } \\
\text { scratching with large surface coverage, or small } \\
\text { localized area of corrosion } \\
\text { Penetrating scratches on }>30 \% \text { surface, faint } \\
\text { scratching with larger surface coverage and }<20 \% \\
\text { areas of deeper gouging into the bulk material, or } \\
\text { area of corrosion on the surface }>30 \% \\
\text { Penetrating scratches on majority }(>50 \%) \text { of } \\
\text { surface, or major area of corrosion }>50 \%\end{array}$ \\
Severe & 3 &
\end{tabular}




\section{Cytokine Assay}

Synovial aspirates were collected from the necropsy knees with well-fixed primary total knee replacement after IRB approval. A magnetic bead-based multiplex assay using the Luminex MAGPIX platform (R\&D Systems, Minneapolis, MN) was performed for simultaneous detection of the following human analytes: TNF- $\alpha$, IL-1 $\beta$, IL-6, IL-8, MCP-1, MIP-1 $\alpha$, MIP-3 $\alpha$, GM-CSF, M-CSF, IFN- $\gamma$, and IL-2. Per the instructions, the standards, calibrator diluent RD6-52, synovial samples, and diluent RD2-1 were brought to room temperature. The Certificate of Analysis provided with the kit was followed in order to reconstitute each standard cocktail with RD6-52 diluent. The reconstituted standards were left under gentle agitation for 15 minutes after which the standards 1 through 6 were created according to the directions in the booklet. The RD652 diluent was then added to dilute each sample by a factor of 2 , using 80 microliters $(\mu \mathrm{L})$ of synovial sample and $80 \mu \mathrm{L}$ if diluent, resulting in $160 \mu \mathrm{L}$ solution in each microparticle cocktail. The lights were turned off and the microparticle cocktail was centrifuged for 30 seconds at 1,000 G. The cocktails were then gently vortexed and then diluted with RD2-1. The standards and microparticle cocktails were added to their corresponding wells on the 96-well plate and covered with a foil seal before incubation for two hours at room temperature on a microplate shaker. During this period, the wash buffer was created by adding $20 \mathrm{~mL}$ wash buffer concentrate to $480 \mathrm{~mL}$ deionized water. After the 2 hours incubation period with the lights still off, a magnet was placed on the bottom of the microplate and the plate was then shaken over a sunk to remove the liquid. Each well was filled with $100 \mu \mathrm{L}$ if wash buffer which was then shaken out. This step was repeated a total of three times. The biotin antibody cocktail was then centrifuged and diluted with the RD2-1 diluent. Each well received $50 \mu \mathrm{L}$ of the diluted biotin cocktail before the microplate was incubated for 1 hour at $800 \mathrm{rpm}$ under a foil seal. The streptavidin cocktail was then prepared by mixing $220 \mu \mathrm{L}$ with $5.35 \mu \mathrm{L}$ of wash buffer. After the 1 hour incubation period, the wells were washed with wash buffer three times in the same manner as discussed previously and then $50 \mu \mathrm{L}$ of the prepared streptavidin cocktail was added to each well. The microplate was then incubated for 30 minutes at room temperature at $800 \mathrm{rpm}$. After the incubation period, the wells were then washed three times again as previously described. Finally, $100 \mu \mathrm{L}$ of wash buffer was added to each well and incubated for 2 minutes at $800 \mathrm{rpm}$ before the plate was analyzed using the Luminex MAGPIX platform which reported each cytokine concentration in picograms per milliliter $(\mathrm{pg} / \mathrm{mL})$.

\section{ICP-MS Analysis}

ICP-MS is an analytical technique used to measure elemental determinations in a material. Tissue samples were collected from the medial and lateral gutters, supra- and infra-patellar regions, and from along the tibia-implant interface. Each of these tissue samples were digested using a modified method from Kerger et al. (201).

Prior to acid digestion to prepare the samples for analysis, the laboratory equipment had to be prepared. An acid solution of 5 to $10 \%$ trace metal nitric acid was combined with 
18 mega ohm ultrapure water to rinse and soak the sample containers, digest vessel, and any equipment that may come into direct contact with samples or fluids during the acid digestion. Minor tools were washed twice and then dried with Kimtech wipes (KimberlyClark Professional, Rosswell, GA) whereas tools that would come in direct contact with the samples were soaked overnight in the solution and then were dried.

After preparing the laboratory space and equipment, the tissue samples were cut to 25 milligrams in size using a ceramic knife and plastic tweezers. Care was taken to ensure the samples did not contact any form of metal. A water bath was set to $95^{\circ}$ Celsius, and centrifuge tubes were labeled to identify the samples. The samples were then washed with 18 mega-ohm ultrapure water to remove ethanol and were placed in the correctly labeled tube. The acid solution was created by adding $2 \mathrm{~mL}$ of $70 \%$ trace metal grade nitric acid to each tube under a fume hood. Next, $1 \mathrm{~mL}$ of hydrogen peroxide was added to each tube followed by $3 \mathrm{~mL}$ of $37 \%$ trace metal grade hydrochloric acid, also under the fume hood. Three samples of only acid solution were also prepared in 3 tubes with no tissue samples as blank samples. The hydrogen peroxide was allowed to react as the water bath heated to the correct temperature. Once heated, the tubes were placed in the water bath for a 2 hours incubation period. After the 2 hours, the tubes were removed and placed in the fume hood for 30 minutes to cool. After the cooling period, an addition $1 \mathrm{~mL}$ of hydrogen peroxide was added to each tube and given 30 minutes to react. The tubes were then placed back into the water bath for an additional incubation period of 2 to 3 hours.

The samples were then placed into labeled $2 \mathrm{~mL}$ micro-centrifuge tubes to be sent to Brooks Applied Labs (Bothell, WA). Each tube received $1 \mathrm{~mL}$ of the sample and acid mixture and $1 \mathrm{~mL}$ of 18 mega-ohm ultrapure water. The remaining sample was diluted to a $15 \mathrm{~mL}$ volume with the 18 mega-ohm ultrapure water. The method for analysis used by Brooks Applied Labs was validated using the Luts-1 certified reference material from the National Research Council of Canada. This material is a solid biological matrix that contains certified quantities of cobalt and chromium.

Once Brooks Applied Labs completed the analysis, the data was first checked to ensure the blank samples had low levels of metal. Any amount of metal in the blank sample was considered to be a contamination. If the contaminants were low in value and consistent between the blanks, they were averaged and the average was subtracted from the remaining samples. This allows the final data to be represented without the background noise present due to uncontrollable contamination. The data was then reported in $\mu \mathrm{g} / \mathrm{L}$.

\section{Statistical Analyses}

Before statistical analyses were performed, the data was analyzed for normality. Using Microsoft Excel (Microsoft, Redmond, WA), a Shapiro-Wilk normality test revealed that the data sets were not normally distributed. As such, these data sets were reported as median and interquartile ranges (IQR). A nonparametric Wilcoxon Signed- 
Rank Test for paired samples was performed on the tibial insert wear and femoral component scores to determine if any significant difference existed between the scores given by the two observers. Additionally, Mann-Whitney rank sum test was performed to determine if significant differences in laxity, particularly AP and IE laxity, existed between PS and CR implants. Because the wear and damage scores were subjective, ordinal data, no correlation analyses were performed between those data sets and the objective, nominal data sets of the laxity, cytokine, and tissue metal measurements. Using SigmaPlot statistical software, the nonparametric Spearman's rank correlation test was performed with an assumed significance of $\alpha=0.05$ to determine if significant correlations existed between the remaining parameters. 


\section{CHAPTER 3. RESULTS}

\section{Clinical and Implant Information}

In total, 35 necropsy specimens were retrieved. However, 2 specimens were excluded from analysis: a unicompartmental implant and a modular revision implant. The final sample size was 33 well-functioning primary TKA specimens. Full clinical information was not available for all implants; the average age at autopsy was 76 years (range: 62 to 94 years of age) and there were 12 males, 22 females, and 1 unknown gender included in the cohort. The implant lifespan in situ when available was included in the summary of the implant information presented in Table 3-1. All femoral components were CoCrMo alloy except for DRLU0104160-R, a Smith \& Nephew (Memphis, TN) Genesis II design with an Oxinium femoral component, and RLU1018161B, a Zimmer (Warsaw, IN) Miller-Galante II design with a TiAlV femoral component. With respect to fixation, 28 femoral components were cemented while 5 were uncemented and well-fixed with porous titanium $(n=1)$, small beads $(n=3)$, or fiber mesh $(n=1)$. The tibial components retrieved in this study included 2 monoblock designs (RLU0722161B-L — an all-polyethylene design and RLU1121161A-L_a compressionmolded, non-modular monoblock design), 18 TiAlV tibial baseplates, and 14 CoCrMo tibial baseplates. With respect to tibial component fixation, 29 tibial trays were cemented while 4 were uncemented and well-fixed with porous titanium $(n=1)$, small beads $(n=2)$, and fiber mesh $(n=1)$. Of the 33 specimens retrieved, 26 were fixed bearing implant systems, 5 were mobile bearing implant systems, and, as previously mentioned, 2 were monoblock implant systems. 15 of the retrieved implants were PS while 18 were CR designs. The sterilization methods of the retrieved tibial inserts included: gamma air $(n=8)$, gamma inert $(n=19)$, ethylene oxide ( $n=3$; Smith \& Nephew, Memphis, TN), X3 $(\mathrm{n}=1$; Stryker, Kalamazoo, MI), ArCom ( $\mathrm{n}=1$; Zimmer Biomet, Warsaw, IN), and Duracon ( $\mathrm{n}=1$; Stryker Howmedica, Kalamazoo, MI). The X3 sterilized insert (RLU0829162C-R) was the only highly-cross linked UHMWPE tibial insert in the cohort. All implants had resurfaced patellas with polyethylene patellar buttons except for specimen DRLU051714-L, a Zimmer Natural Knee implant system (Warsaw, IN).

\section{Implant Wear and Damage Assessment}

A nonparametric Wilcoxon Signed-Rank Test for paired samples revealed that there was no significant difference between the two scorers of the femoral component damage or polyethylene wear analysis. Thirty-two tibial inserts were available to be assessed for wear on the condylar, backside, post surface (when applicable) as shown in Table 3-2, which includes the sterilization technique of each tibial insert. As previously stated, the maximum condylar wear score that could be given was a score of 168 while the maximum possible score for each of the 7 damage modes was 24 . The median wear score for the condylar region of the insert was a score of 38 (IQR: 11), with a range of scores from 27 (RLU0426162B-R, shown in Figure 3-1) to 64 (DRLU081315-R). 
Table 3-1. Implant information of study cohort including manufacturer, design, and time in situ (years)

\begin{tabular}{|c|c|c|c|}
\hline Specimen & Manufacturer & Design & Time in Situ \\
\hline DRLU051714-L & Zimmer & Natural Knee & $>10$ \\
\hline DRLU081315-R & Zimmer & Nexgen Legacy PS (LPS) & 20 \\
\hline DRLU030815-R & Zimmer & Nexgen LPS Flex & 15 \\
\hline DRLU121515-R & Zimmer & Nexgen LPS Flex & $>10$ \\
\hline DRLU010416-R & Smith \& Nephew & Genesis II & 21 \\
\hline DRLU112815-R & Stryker & Scorpio & 11 \\
\hline DRLU101615-R & Stryker & Scorpio Flex CR & 22 \\
\hline RLU1016151A-L & Stryker & Scorpio & 21 \\
\hline 2013-678L & Depuy & Low Contact Stress (LCS) Mobile Bearing & -- \\
\hline RLU0510162A-R & Zimmer & Nexgen LPS Flex & 7 \\
\hline RLU0728161A-L & Smith \& Nephew & Unknown & 27 \\
\hline RLU0309161B-L & Depuy & Anatomic Modular Knee (AMK) & 45 \\
\hline RLU0722161B-L & Cintor Division of Codman & Unknown & 40 \\
\hline RLU0126161C-L & Smith \& Nephew & Genesis II & -- \\
\hline RLU0510161A-L & Zimmer & Nexgen LPS Flex & 6 \\
\hline RLU0426161B-L & Depuy & LCS Mobile Bearing & -- \\
\hline RLU0126162C-R & Smith \& Nephew & Genesis II & -- \\
\hline RLU0720161B-L & Depuy & Press Fit Condylar (PFC) Sigma & 13 \\
\hline RLU1028161B-L & Zimmer & Miller-Galante (MG) II & 37 \\
\hline RLU1115162B-R & Zimmer & Nexgen LPS Flex & 11 \\
\hline RLU0913162A-R & Zimmer & Insall Burnstein (IB) PS II & 45 \\
\hline RLU0426162B-R & Depuy & LCS Mobile Bearing & -- \\
\hline RLU0829162C-R & Stryker & Triathlon CR & $<11$ \\
\hline $16-04-765 \mathrm{R}$ & Biomet & Vanguard & -- \\
\hline $16-10-1103 R$ & Depuy & Sigma Mobile Bearing & -- \\
\hline $16-10-1103 \mathrm{~L}$ & Depuy & Sigma Mobile Bearing & -- \\
\hline RLU0204172A-R & Stryker & Triathlon PS & 19 \\
\hline RLU0317172B-R & Stryker & Triathlon CR & 15 \\
\hline RLU0319171A-L & Stryker Howmedica & Duracon & 15 \\
\hline RLU1121161A-L & Biomet & Anatomical Graduated Component (AGC) & 27 \\
\hline RLU1121162A-R & Johnson \& Johnson & Press Fit Condylar (PFC) & 27 \\
\hline RLU1125161A-L & Zimmer & Natural Knee & 22 \\
\hline RLU1125162A-R & Zimmer & Natural Knee & 21 \\
\hline
\end{tabular}


Table 3-2. Summary of total tibial insert wear scores by location

\begin{tabular}{|c|c|c|c|c|}
\hline Specimen & Condyle & Backside & Post & $\begin{array}{c}\text { Sterilization } \\
\text { Method }\end{array}$ \\
\hline DRLU051714-L & 27 & 44 & -- & Gamma Inert \\
\hline DRLU081315-R & 64 & 34 & 22 & Gamma Inert \\
\hline DRLU030815-R & 31 & 48 & 2 & Gamma Inert \\
\hline DRLU121515-R & 36 & 46 & 7 & Gamma Inert \\
\hline DRLU010416-R & 47 & $\mathrm{U} / \mathrm{A}$ & -- & $\mathrm{EtO}$ \\
\hline DRLU112815-R & 32 & 31 & 5 & Gamma Inert \\
\hline DRLU101615-R & $\mathrm{U} / \mathrm{A}$ & 16 & -- & Gamma Inert \\
\hline RLU1016151A-L & 51 & 19 & -- & Gamma Air \\
\hline 2013-678L & 42 & 41 & -- & Gamma Inert \\
\hline RLU0510162A-R & 42 & 57 & 12 & Gamma Inert \\
\hline RLU0728161A-L & 36 & 21 & -- & Gamma Air \\
\hline RLU0309161B-L & 37 & 32 & -- & Gamma Air \\
\hline RLU0722161B-L & 42 & $\mathrm{U} / \mathrm{A}$ & -- & Gamma Air \\
\hline RLU0126161C-L & 42 & 18 & 6 & EtO \\
\hline RLU0510161A-L & 38 & 52 & 9 & Gamma Inert \\
\hline RLU0426161B-L & 42 & 32 & -- & Gamma Inert \\
\hline RLU0126162C-R & 36 & 17 & 5 & EtO \\
\hline RLU0720161B-L & 29 & 23 & -- & Gamma Inert \\
\hline RLU1028161B-L & 57 & 84 & -- & Gamma Air \\
\hline RLU1115162B-R & 31 & 25 & 2 & Gamma Inert \\
\hline RLU0913162A-R & 58 & 56 & 16 & Gamma Air \\
\hline RLU0426162B-R & 31 & 59 & -- & Gamma Inert \\
\hline RLU0829162C-R & 41 & 23 & -- & $\mathrm{X} 3$ \\
\hline $16-04-765 \mathrm{R}$ & 44 & 13 & 8 & ArCom \\
\hline $16-10-1103 R$ & 30 & 33 & 9 & Gamma Inert \\
\hline 16-10-1103L & 40 & 57 & 12 & Gamma Inert \\
\hline RLU0204172A-R & 33 & 16 & 7 & Gamma Inert \\
\hline RLU0317172B-R & 43 & 12 & -- & Gamma Inert \\
\hline RLU0319171A-L & 35 & 11 & -- & Duration \\
\hline RLU1121161A-L & 54 & $\mathrm{U} / \mathrm{A}$ & -- & Gamma Air \\
\hline RLU1121162A-R & 54 & $\mathrm{U} / \mathrm{A}$ & 15 & Gamma Air \\
\hline RLU1125161A-L & 45 & 32 & -- & Gamma Inert \\
\hline RLU1125162A-R & 41 & 8 & -- & Gamma Inert \\
\hline Median & 41 & 32 & 8 & \\
\hline IQR & 9 & 28 & 7 & \\
\hline
\end{tabular}

Data points denoted by “--" signifies that the implant in question was a CR design that would not have a post

"U/A" signifies that those scores were unavailable 


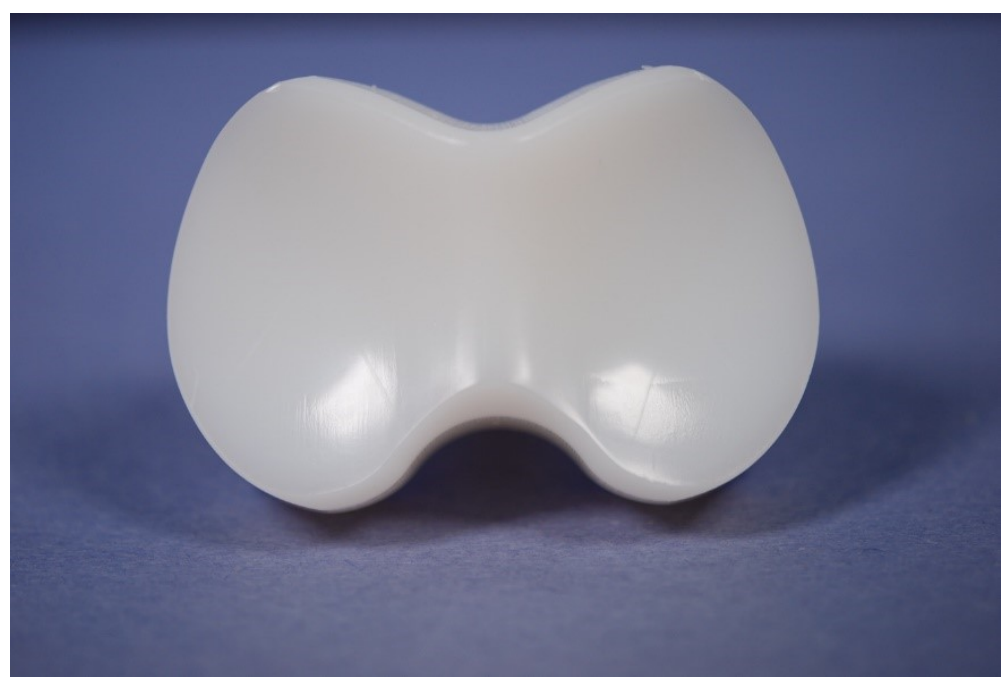

Figure 3-1. Specimen RLU0426162B-R tibial insert

Note: The implant received a score of 27 out 168 and had least amount of total condylar wear. 
Burnishing was the most common type of damage with a median score of 20 (IQR: 4, range: 15 to 24). Pitting and scratching followed with median scores of 8 (IQR: 5 , range 4 to 23) and 9 (IQR: 3, range: 4 to 17), respectively. Two implants had moderate to severe delamination with total scores of 17 (RLU1016151A-L) and 22 (RLU1028161B-L, shown in Figure 3-2), while the rest demonstrated minimal delamination of the condylar surface. Very few of the implants had minimal abrasion, surface deformation, or embedded debris.

The maximum backside wear score that could be given was a score of 168 with a maximum possible score of 24 for each of the 7 damage modes. The median wear score for the backside region of the insert was a score of 32 (IQR: 28), with a range of scores from 13 (16-04-765R) to 84 (RLU1028161B-L). Again, burnishing was the most common mode of damage with a median score of 9 (IQR: 15, range 0 to 24). Scratching and pitting followed with median scores of 7 (IQR: 8, range 0 to 24) and (IQR: 9, range: 2 to 24). Fifteen implants demonstrated mild to moderate backside surface deformation with a median score of 9 (IQR: 4, range: 3 to 15). One specimen had moderate delamination with a score of 12 , but no specimens had backside abrasion or embedded debris.

As previously mentioned, 15 of the 32 retrieved TKAs were PS designs. The 7 wear modes were assessed on this additional interface to determine if the stabilizing post contributes to the production of polyethylene debris. The maximum possible wear score for the post of each PS implant was a score of 84 while each wear mode had a possible maximum score of 12 . The median wear score of the stabilizing post was a score of 8 (IQR: 7), with a range from 2 (RLU1115162B-R and DRLU030815-R) to 22 (DRLU081315-R). The most prominent wear mode was burnishing with a median score of 6 (IQR: 4, range 0 to 10). Specimen RLU1121162A-R demonstrated severe wear on the anterior surface of the stabilizing post, as shown in Figure 3-3, and received a total wear score of 15 for the post surface. The remainder of the wear modes were nonexistent or minimal on the stabilizing post surfaces.

Twenty-nine femoral components were analyzed for damage. For each component, the maximum possible damage score was 16 . The median damage score was a score of 5 with an interquartile range of 4 . Minimal damage (score of 1) was observed in $31 \%(n=9 / 29)$ femoral components. The femoral component of specimen RLU0829162C-R, shown in Figure 3-4, was among those with minimal damage with a total score of 4 . Mild damage (score of 2$)$ was observed in $45 \%(n=13 / 29)$ femoral components. Moderate damage (score of 3$)$ was observed in $21 \%(n=6 / 29)$ femoral components. Severe damage (score of 4 ) was observed on 1 of the $29(3 \%)$ femoral components. The femoral component of specimen RLU1028161B-L had the most severe damage with a score of 14, shown in Figure 3-5. This implant exhibits severe scratching along both its medial and lateral bearing surface which garnered damage scores of 3 for the lateral and medial anterior surface and damage scores of 4 for the lateral and medial posterior surfaces. Of the study samples, $70 \%$ of the specimens demonstrated mild to severe damage in at least one quadrant (score $\geq 2$ ). 


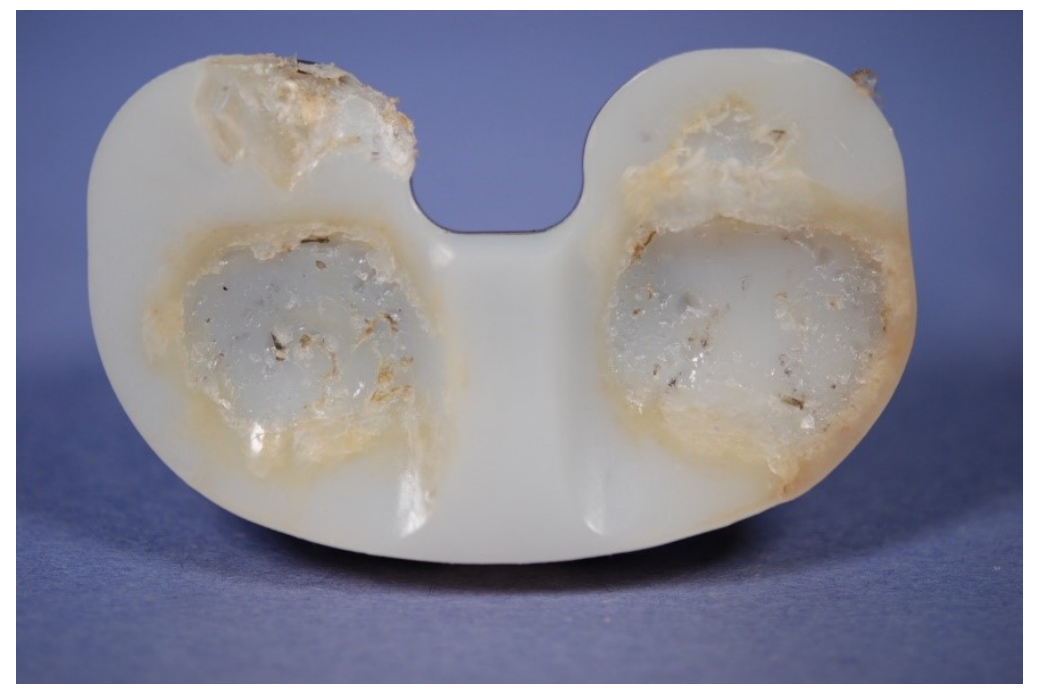

Figure 3-2. Specimen RLU1028161B-L tibial insert

Note: The implant received a score of 22 out of 24 and demonstrated severe delamination accompanied by oxidation. 


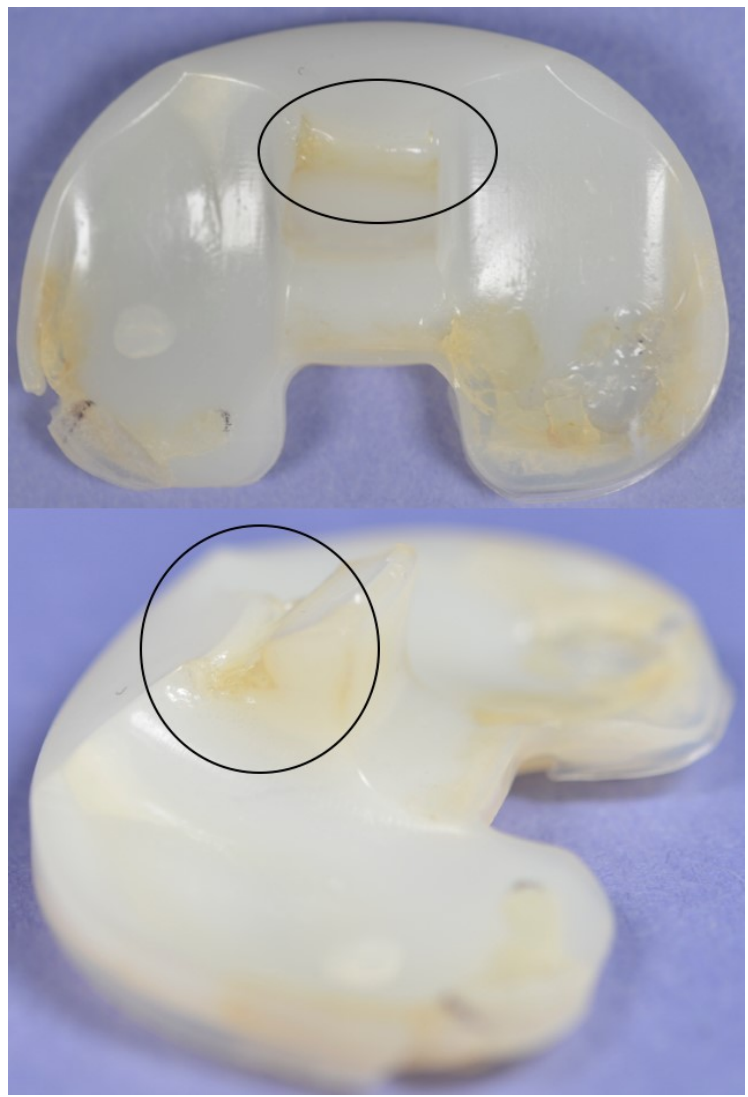

Figure 3-3. Specimen RLU1121162A-R tibial insert

Note: Tibial insert post demonstrates severe anterior impingement wear. 


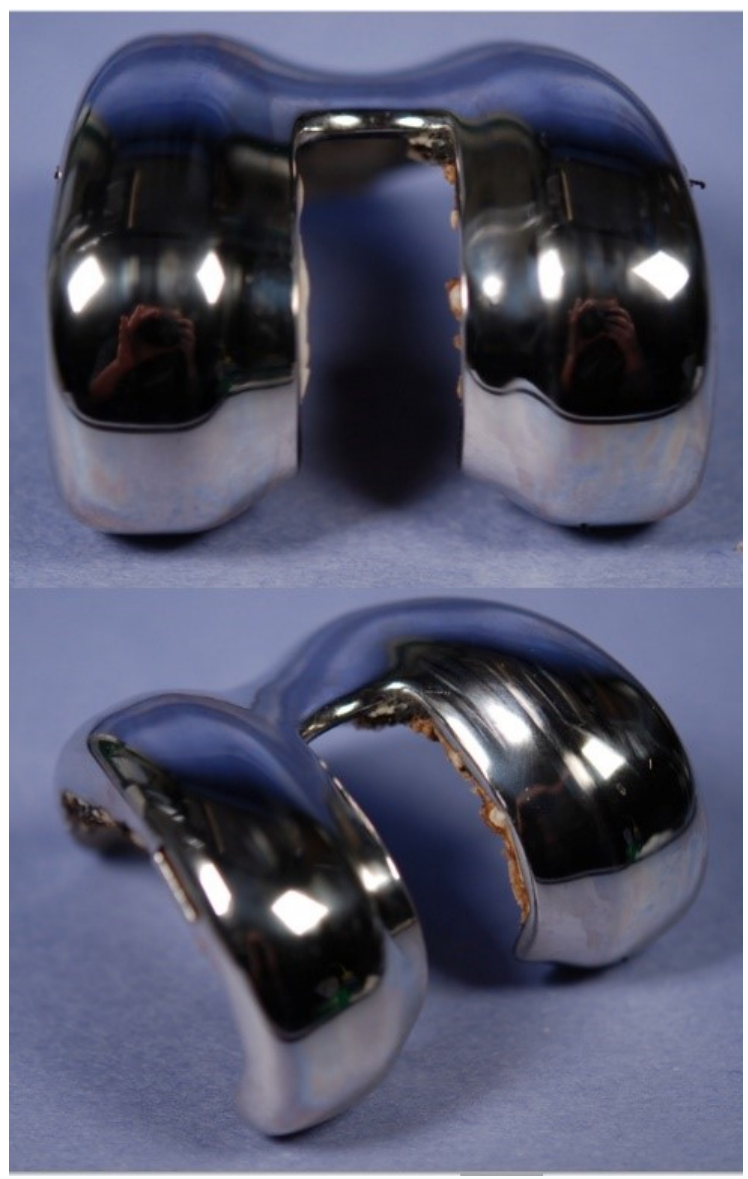

Figure 3-4. Specimen RLU0829162C-R femoral component

Note: This implant exhibited minimal damage with lowest possible total score of 4 . 


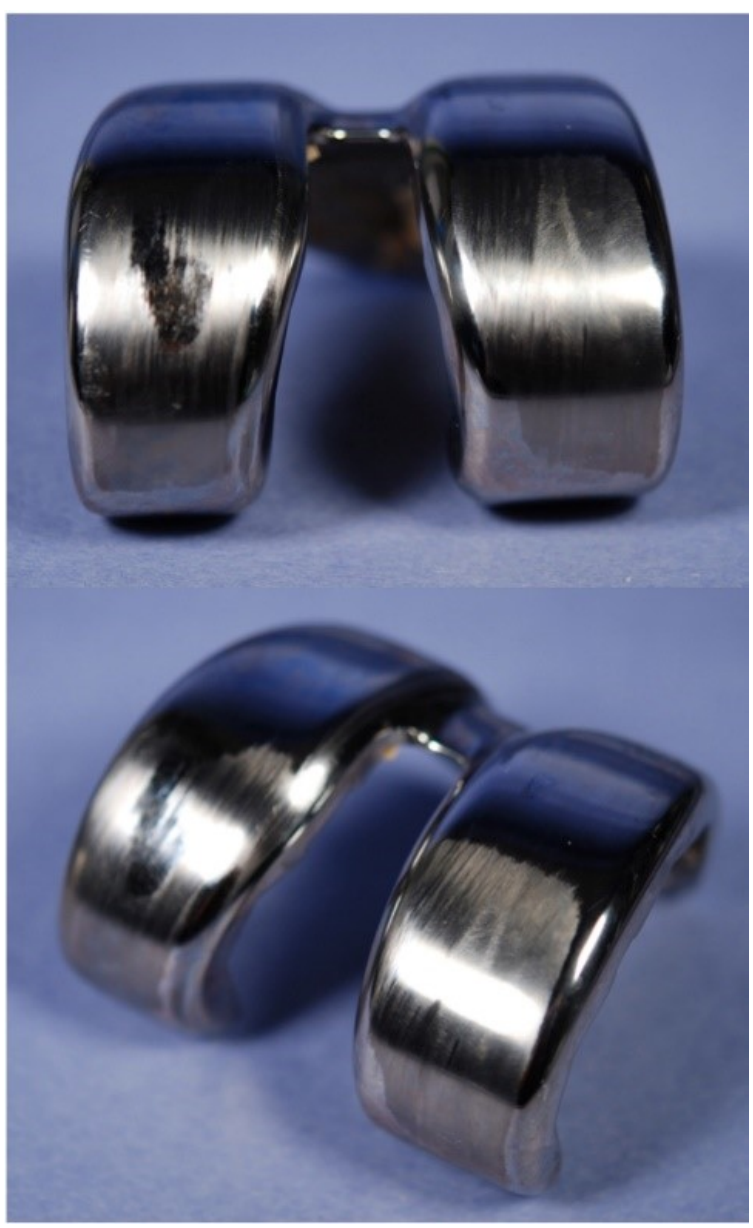

Figure 3-5. Specimen RLU1028161B-L femoral component

Note: This implant exhibited severe damage with a total score of 14 out of 16 . 


\section{Laxity}

Laxity profiles were measured for 23 cadaveric primary TKA specimens. Specimens exhibited large variations in laxity. Some specimens were not able to reach full extension or $90^{\circ}$ of flexion. All laxity values reported in this discussion and in the tables follow the Grood and Suntay sign conventions. Mann-Whitney rank sum test was performed to determine if significant differences in laxity existed between PS and CR implants.

As shown in Table 3-3, internal rotation ranged from -4.59 to $-29.11^{\circ}$ at full extension, -8.99 to $-31.39^{\circ}$ at $30^{\circ}$ flexion, -1.92 to $-35.05^{\circ}$ at $60^{\circ}$ flexion, and -4.70 to $42.92^{\circ}$ at $90^{\circ}$ flexion. External rotation ranged from 4.01 to $28.05^{\circ}$ at full extension, 11.70 to $32.76^{\circ}$ at $30^{\circ}$ flexion, 1.59 to $39.82^{\circ}$ at $60^{\circ}$ flexion, and 1.24 to $44.62^{\circ}$ at $90^{\circ}$ flexion. At full extension and $90^{\circ}$ of flexion, the median internal rotational laxity was $0.53^{\circ}$ and $0.88^{\circ}$ greater than the median external rotational laxity, whereas, at $30^{\circ}$ and $60^{\circ}$ of flexion, the median external rotational laxity was $0.28^{\circ}$ and $0.72^{\circ}$ greater than the median internal rotational laxity, respectively. Mann-Whitney rank sum test revealed significant differences in the measured external laxity at full extension $(\mathrm{p}=0.01)$ and $30^{\circ}$ of flexion $(\mathrm{p}=0.04)$ between the PS and CR implants. At full extension, the PS specimens had a median external rotation of $14.16^{\circ}$ (IQR: $5.02^{\circ}$ ) while the CR specimens had a median external rotation of $7.43^{\circ}$ (IQR: $2.07^{\circ}$ ). At $30^{\circ}$ of flexion, the PS specimens had a median external rotation of $20.35^{\circ}$ (IQR: $5.07^{\circ}$ ) while the CR specimens had a median external rotation of $15.70^{\circ}$ (IQR: $3.99^{\circ}$ ). There were not any statistically significant differences in internal or external rotation at the remaining flexion angles between the PS and CR implants.

As shown in Table 3-4, varus deflection ranged from -1.07 to $-10.76^{\circ}$ at full extension, -3.24 to $-13.53^{\circ}$ at $30^{\circ}$ flexion, -2.71 to $-18.12^{\circ}$ at $60^{\circ}$ flexion, and -3.24 to $25.26^{\circ}$ at $90^{\circ}$ flexion. Valgus deflection ranged from 0.61 to $7.98^{\circ}$ at full extension, 2.29 to $14.90^{\circ}$ at $30^{\circ}$ flexion, 1.67 to $26.57^{\circ}$ at $60^{\circ}$ flexion, and 2.68 to $38.49^{\circ}$ at $90^{\circ}$ flexion. At $0^{\circ}, 30^{\circ}, 60^{\circ}, 90^{\circ}$, the median varus laxity was $1.22^{\circ}, 3.25^{\circ}, 2.16^{\circ}$, and $3.58^{\circ}$ greater than the median valgus laxity, respectively. Mann-Whitney rank sum test did not reveal any significant differences in varus or valgus deflection across the flexion angles between the PS and CR implants.

As shown in Table 3-5, anterior displacement ranged from 0.13 to $9.37 \mathrm{~mm}$ at full extension, 0.54 to $7.69 \mathrm{~mm}$ at $30^{\circ}$ flexion, 0.01 to $10.77 \mathrm{~mm}$ at $60^{\circ}$ flexion, and 0.01 to $6.51 \mathrm{~mm}$ at $90^{\circ}$ flexion. Posterior displacement ranged from -1.06 to $-6.64 \mathrm{~mm}$ at full extension, -0.25 to $-24.23 \mathrm{~mm}$ at $30^{\circ}$ flexion, -0.16 to $-16.91 \mathrm{~mm}$ at $60^{\circ}$ flexion, and -0.04 to $-18.04 \mathrm{~mm}$ at $90^{\circ}$ flexion. At $0^{\circ}, 30^{\circ}, 60^{\circ}, 90^{\circ}$, the median posterior displacement was $0.51,0.34,1.41$, and $1.49 \mathrm{~mm}$ greater than the median anterior displacement, respectively. Mann-Whitney rank sum test revealed a significant difference in the measured posterior laxity at $30^{\circ}$ of flexion between the PS and CR implants ( $\left.\mathrm{p}=0.007\right)$. The PS specimens had a median posterior displacement of $-9.83 \mathrm{~mm}$ (IQR: $7.29 \mathrm{~mm}$ ) 
Table 3-3. Summary of internal and external rotational laxity at each flexion angle for each specimen

\begin{tabular}{|c|c|c|c|c|c|c|c|c|}
\hline \multirow[b]{2}{*}{ Specimen } & \multicolumn{4}{|c|}{ Internal Rotation $\left({ }^{\circ}\right)$} & \multicolumn{4}{|c|}{ External Rotation $\left({ }^{\circ}\right)$} \\
\hline & $\mathbf{0}^{\circ}$ & $30^{\circ}$ & $60^{\circ}$ & $90^{\circ}$ & $\mathbf{0}^{\circ}$ & $30^{\circ}$ & $60^{\circ}$ & $90^{\circ}$ \\
\hline RLU0510162A-R & -16.62 & -17.62 & -24.47 & -25.17 & 14.16 & 20.35 & 20.59 & 22.68 \\
\hline RLU0728161A-L & -6.81 & -13.28 & -18.00 & -24.60 & 7.43 & 15.60 & 22.42 & 26.29 \\
\hline RLU0722161B-L & -11.01 & -17.09 & -1.92 & --- & 11.35 & 13.02 & 1.59 & --- \\
\hline RLU0126161C-L & -20.56 & -22.77 & -20.71 & --- & 15.50 & 20.44 & 22.52 & --- \\
\hline RLU0510161A-L & -14.16 & -13.02 & -15.10 & -17.65 & 12.69 & 22.92 & 22.12 & 20.93 \\
\hline RLU0426161B-L & -7.09 & -18.55 & -21.73 & -21.81 & 7.43 & 18.37 & 19.38 & 20.97 \\
\hline RLU0126162C-R & -6.48 & -15.69 & -16.98 & -18.50 & 11.62 & 18.97 & 18.27 & 16.51 \\
\hline RLU0720161B-L & -7.51 & -16.82 & -19.52 & -19.21 & 8.22 & 17.37 & 17.77 & 17.28 \\
\hline RLU1028161B-L & --- & -8.99 & -9.23 & -14.12 & --- & 12.01 & 9.40 & 17.37 \\
\hline RLU1115162B-R & -5.76 & -13.17 & -16.44 & -9.71 & 4.01 & 11.70 & 14.06 & 15.48 \\
\hline RLU0913162A-R & -4.59 & -10.84 & -3.48 & -21.28 & 28.05 & 25.20 & 39.82 & 35.88 \\
\hline RLU0426162B-R & -11.29 & -19.36 & -21.69 & -25.30 & 15.46 & 21.68 & 23.58 & 16.96 \\
\hline RLU0829162C-R & -9.96 & -21.11 & -22.79 & -27.01 & 5.92 & 15.80 & 18.67 & 20.06 \\
\hline 16-04-765R & -16.06 & -12.69 & -12.28 & -4.70 & 13.59 & 21.10 & 7.89 & 1.24 \\
\hline $16-10-1103 R$ & -13.71 & -16.70 & -17.91 & -12.24 & 17.71 & 17.29 & 12.61 & 13.42 \\
\hline $16-10-1103 \mathrm{~L}$ & -29.11 & -31.39 & -35.05 & -38.24 & 24.49 & 32.76 & 33.74 & 37.38 \\
\hline RLU0204172A-R & --- & -11.58 & -14.27 & -17.24 & --- & 12.49 & 16.45 & 18.52 \\
\hline RLU0317172B-R & -7.20 & -17.33 & -18.26 & -24.07 & 6.25 & 15.48 & 20.82 & 27.38 \\
\hline RLU0319171A-L & -11.67 & -15.99 & -23.45 & -26.38 & 6.64 & 13.78 & 15.37 & 16.16 \\
\hline RLU1121161A-L & -10.41 & -20.73 & -25.60 & -28.78 & 6.58 & 12.19 & 20.24 & 30.23 \\
\hline RLU1121162A-R & --- & -18.59 & -26.96 & -42.92 & --- & 16.60 & 25.88 & 44.62 \\
\hline RLU1125161A-L & -12.94 & -18.06 & -29.44 & -36.75 & 8.37 & 17.58 & 25.05 & 34.31 \\
\hline RLU1125162A-R & -9.09 & -19.99 & -28.79 & -5.20 & 9.00 & 17.59 & 32.25 & 30.13 \\
\hline Median & -10.71 & -17.09 & -19.52 & -21.81 & 10.18 & 17.37 & 20.24 & 20.93 \\
\hline IQR & 6.65 & 5.70 & 8.19 & 9.14 & 7.26 & 5.76 & 7.14 & 13.18 \\
\hline
\end{tabular}


Table 3-4. Summary of varus and valgus laxity at each flexion angle for each specimen

\begin{tabular}{|c|c|c|c|c|c|c|c|c|}
\hline \multirow[b]{2}{*}{ Specimen } & \multicolumn{4}{|c|}{ Varus Deflection $\left({ }^{\circ}\right)$} & \multicolumn{4}{|c|}{ Valgus Deflection $\left({ }^{\circ}\right)$} \\
\hline & $\mathbf{0}^{\circ}$ & $30^{\circ}$ & $60^{\circ}$ & $90^{\circ}$ & $\mathbf{0}^{\circ}$ & $30^{\circ}$ & $60^{\circ}$ & $90^{\circ}$ \\
\hline RLU0510162A-R & -3.64 & -5.12 & -4.47 & -11.96 & 3.10 & 3.76 & 6.12 & 3.06 \\
\hline RLU0728161A-L & -2.64 & -3.90 & -8.43 & -3.24 & 2.24 & 4.08 & 4.70 & 3.19 \\
\hline RLU0722161B-L & -6.54 & -9.20 & -2.71 & --- & 3.95 & 6.52 & 1.67 & --- \\
\hline RLU0126161C-L & -6.24 & -7.91 & -11.98 & --- & 3.55 & 5.73 & 7.89 & --- \\
\hline RLU0510161A-L & -6.72 & -7.06 & -6.43 & -9.27 & 0.61 & 2.96 & 5.14 & 3.95 \\
\hline RLU0426161B-L & -4.64 & -8.99 & -9.19 & -10.38 & 3.32 & 3.34 & 6.34 & 6.75 \\
\hline RLU0126162C-R & -3.82 & -6.55 & -8.02 & -11.57 & 3.64 & 4.25 & 6.82 & 5.45 \\
\hline RLU0720161B-L & -3.23 & -9.69 & -10.27 & -6.74 & 1.77 & 3.75 & 4.26 & 9.53 \\
\hline RLU1028161B-L & --- & -7.25 & -8.00 & -7.99 & --- & 4.18 & 6.57 & 8.64 \\
\hline RLU1115162B-R & -4.03 & -7.50 & -8.53 & -11.64 & 1.63 & 4.73 & 7.43 & 5.84 \\
\hline RLU0913162A-R & -7.12 & -9.26 & -18.12 & -13.16 & 2.77 & 3.84 & 6.46 & 19.68 \\
\hline RLU0426162B-R & -8.56 & -8.65 & -8.36 & -4.46 & 3.97 & 5.09 & 5.88 & 4.82 \\
\hline RLU0829162C-R & -9.10 & -10.10 & -13.29 & -22.89 & 4.04 & 5.52 & 10.69 & 9.01 \\
\hline 16-04-765R & -3.89 & -9.15 & -8.51 & -4.00 & 7.98 & 4.43 & 5.33 & 2.85 \\
\hline $16-10-1103 R$ & -5.32 & -7.51 & -8.53 & -9.36 & 2.89 & 2.57 & 4.36 & 3.25 \\
\hline $16-10-1103 \mathrm{~L}$ & -10.76 & -13.53 & -17.29 & -15.93 & 7.54 & 9.61 & 13.28 & 24.22 \\
\hline RLU0204172A-R & --- & -3.24 & -7.61 & -9.47 & --- & 2.82 & 4.73 & 7.63 \\
\hline RLU0317172B-R & -2.47 & -5.14 & -6.30 & -13.15 & 2.05 & 2.29 & 5.52 & 8.35 \\
\hline RLU0319171A-L & -1.07 & -3.68 & -4.43 & -9.80 & 5.41 & 6.24 & 8.59 & 14.42 \\
\hline RLU1121161A-L & -2.17 & -5.52 & -9.74 & -15.92 & 2.26 & 5.09 & 11.87 & 13.29 \\
\hline RLU1121162A-R & --- & -12.69 & -14.59 & -25.26 & --- & 14.90 & 9.42 & 38.49 \\
\hline RLU1125161A-L & -9.34 & -6.57 & -3.18 & -10.34 & 3.84 & 4.04 & 2.56 & 6.20 \\
\hline RLU1125162A-R & -4.68 & -8.21 & -9.72 & -9.15 & 5.28 & 6.92 & 8.74 & 2.68 \\
\hline Median & -4.66 & -7.51 & -8.51 & -10.34 & 3.44 & 4.25 & 6.34 & 6.75 \\
\hline IQR & 3.28 & 3.13 & 2.98 & 4.00 & 1.73 & 1.87 & 3.72 & 5.57 \\
\hline
\end{tabular}


Table 3-5. Summary of anterior and posterior laxity at each flexion angle for each specimen

\begin{tabular}{|c|c|c|c|c|c|c|c|c|}
\hline \multirow[b]{2}{*}{ Specimen } & \multicolumn{4}{|c|}{ Anterior Displacement (mm) } & \multicolumn{4}{|c|}{ Posterior Displacement (mm) } \\
\hline & $\mathbf{0}^{\circ}$ & $30^{\circ}$ & $60^{\circ}$ & $90^{\circ}$ & $\mathbf{0}^{\circ}$ & $30^{\circ}$ & $60^{\circ}$ & $90^{\circ}$ \\
\hline RLU0510162A-R & --- & --- & --- & --- & $\begin{array}{ll}-- \\
--\end{array}$ & --- & --- & --- \\
\hline RLU0728161A-L & --- & --- & --- & --- & --- & --- & --- & --- \\
\hline RLU0722161B-L & 4.54 & 3.14 & 0.79 & --- & -2.89 & -7.41 & -0.24 & --- \\
\hline RLU0126161C-L & 2.49 & 3.25 & 0.27 & --- & -2.28 & -1.26 & -0.26 & --- \\
\hline RLU0510161A-L & 3.51 & 4.72 & 2.21 & 2.33 & -3.02 & -5.50 & -0.92 & -7.94 \\
\hline RLU0426161B-L & 2.68 & 5.35 & 2.71 & 1.60 & -3.64 & -3.23 & -6.24 & -9.02 \\
\hline RLU0126162C-R & 3.47 & 7.69 & 4.59 & 1.46 & -3.38 & -9.40 & -3.34 & -4.87 \\
\hline RLU0720161B-L & 1.00 & 5.34 & 3.48 & 2.48 & -1.24 & -4.57 & -5.12 & -4.52 \\
\hline RLU1028161B-L & --- & 5.07 & 1.65 & 1.62 & --- & -0.25 & -3.64 & -2.95 \\
\hline RLU1115162B-R & 2.44 & 7.29 & 1.94 & 1.44 & -1.45 & -13.37 & -7.22 & -3.02 \\
\hline RLU0913162A-R & 9.37 & 5.34 & 6.33 & 6.51 & -5.63 & -10.26 & -14.77 & -8.09 \\
\hline RLU0426162B-R & 0.13 & 0.54 & 1.62 & 0.06 & -2.60 & -2.89 & -1.13 & -0.11 \\
\hline RLU0829162C-R & 1.12 & 2.31 & 0.01 & 0.04 & -2.12 & -1.40 & -0.22 & -0.04 \\
\hline 16-04-765R & 3.94 & 4.77 & 2.35 & 0.35 & -4.44 & -10.65 & -3.94 & -0.35 \\
\hline $16-10-1103 R$ & 1.99 & 3.99 & 2.41 & 0.88 & -3.56 & -5.37 & -3.20 & -1.61 \\
\hline $16-10-1103 \mathrm{~L}$ & 1.82 & 5.18 & 5.16 & 1.86 & -6.64 & -24.23 & -9.30 & -11.44 \\
\hline RLU0204172A_R & --- & 3.73 & 0.37 & 2.12 & --- & -4.58 & -4.94 & -0.54 \\
\hline RLU0317172B_R & 3.59 & 2.98 & 1.36 & 1.86 & -1.06 & -1.68 & -0.16 & -1.14 \\
\hline RLU0319171A_L & 3.99 & 5.51 & 4.70 & 0.94 & -2.14 & -5.06 & -16.91 & -8.95 \\
\hline RLU1121161A_L & 1.78 & 2.55 & 10.77 & 3.27 & -3.62 & -8.31 & -9.21 & -18.04 \\
\hline RLU1121162A_R & --- & 2.25 & 0.34 & 0.40 & --- & -21.16 & -0.80 & -1.98 \\
\hline RLU1125161A_L & 2.64 & 2.25 & 1.93 & 0.01 & -3.14 & -2.62 & -1.18 & -0.26 \\
\hline RLU1125162A_R & 1.83 & 5.35 & 0.69 & 0.02 & -4.43 & -0.46 & -0.32 & -0.28 \\
\hline Median & 2.57 & 4.72 & 1.94 & 1.49 & -3.08 & -5.06 & -3.34 & -2.95 \\
\hline IQR & 1.74 & 2.36 & 2.69 & 1.62 & 1.46 & 6.78 & 5.45 & 7.57 \\
\hline
\end{tabular}


while the CR specimens had a median posterior displacement of $-2.89 \mathrm{~mm}$ (IQR: 3.28 $\mathrm{mm})$. There were not any statistically significant differences in anterior or posterior displacement at the remaining flexion angles between the PS and CR implants.

\section{Inflammatory Cytokines}

The inflammatory cytokine concentrations in the synovial fluid of 32 specimens were measured using two separate cytokine assays, as presented in Table 3-6. The first assay screened for TNF- $\alpha$, IL-1 $\beta$, IL-6, IL-8, MCP-1, MIP-1 $\alpha$, MIP-3 $\alpha$, GM-CSF, MCSF, IFN- $\gamma$, and IL-2. In the second assay, MIP-1 $\alpha$, GM-CSF, and IFN- $\gamma$ were omitted because none of the specimens had concentrations above the detectable limit in the first assay (MIP-1 $\alpha<181 \mathrm{pg} / \mathrm{mL}, \mathrm{GM}-\mathrm{CSF}<11.9 \mathrm{pg} / \mathrm{mL}$, and IFN- $\gamma<299 \mathrm{pg} / \mathrm{mL}$ ).

The range of detection for TNF- $\alpha$ was 8.9 to $2,170 \mathrm{pg} / \mathrm{mL} ; 8$ specimens were below the minimum detection limit for TNF- $\alpha(<8.9 \mathrm{pg} / \mathrm{mL})$ while the remaining 14 specimens were barely about the minimum detection limit. The range of detection for IL$1 \beta$ was 16.3 to $3,950 \mathrm{pg} / \mathrm{mL} ; 3$ specimens were below the minimum detection limit for IL-1 $\beta(<16.3)$. The range of detection for IL- 6 was 4.9 to $1,180 \mathrm{pg} / \mathrm{mL} ; 5$ specimens were above the maximum detection limit for IL-6 $(>1,180 \mathrm{pg} / \mathrm{mL})$. The detection range for IL8 was 4.7 to $1,140 \mathrm{pg} / \mathrm{mL}$; all but 2 of the specimens had concentrations of IL-8 that exceeded the maximum detection limit of the assay platform $(>1,140 \mathrm{pg} / \mathrm{mL})$. The range of detection for MCP-1 was 98 to $7,940 \mathrm{pg} / \mathrm{mL} ; 7$ specimens were above the maximum detection limit for MCP-1 (>7,940 pg/mL). The detection range for MIP-3 $\alpha$ was 7.9 to $1,920 \mathrm{pg} / \mathrm{mL} ; 2$ specimens had concentrations above the maximum detection limit $(>1,920 \mathrm{pg} / \mathrm{mL})$ while 1 specimen was below the minimum detection limit $(<7.9 \mathrm{pg} / \mathrm{mL})$. All specimens had concentrations of M-CSF that were within the detection range, 514 to $124,810 \mathrm{pg} / \mathrm{mL}$. The minimum and maximum M-CSF concentration were $5,566 \mathrm{pg} / \mathrm{mL}$ and $63,135 \mathrm{pg} / \mathrm{mL}$, respectively.

\section{Tissue Metal Concentrations}

Periprosthetic tissues from 23 necropsy specimens were available for ICP-MS analysis, as shown in Table 3-7 which includes the femoral component and tibial baseplate materials. Statistical analysis revealed no significant difference between the tissue locations and the metal concentrations; the concentrations were reported as averages of all five tissue collection locations: the supra- and infra-patellar regions, the medial and lateral gutters, and the tibial tuberosity. Specimens DRLU081315-R and RLU1028161B-L had elevated concentrations of Ti in their periprosthetic tissues $(1,136.3 \mu \mathrm{g} / \mathrm{L}$ and $12,775.1 \mu \mathrm{g} / \mathrm{L}$, respectively). Specimen RLU1028161B-L was an uncemented TKA with Ti fiber mesh coating on the femoral component, the tibial tray, and the metal-backing of the patellar button. Specimen RLU0426161B-L had extremely elevated tissue concentrations of $\operatorname{Co}(28,705.3 \mu \mathrm{g} / \mathrm{L})$ and $\mathrm{Cr}(66,532.2 \mu \mathrm{g} / \mathrm{L})$; this specimen also presented with severe metallosis, as shown previously in Figure 1-5. 
Table 3-6. Concentrations of inflammatory cytokines for cadaveric specimens $(\mathrm{pg} / \mathrm{mL})$

\begin{tabular}{|c|c|c|c|c|c|c|c|c|}
\hline Specimen & TNF- $\alpha$ & IL-1 $\beta$ & IL-6 & IL-8 & MCP-1 & MIP-3a & M-CSF & IL-2 \\
\hline DRLU051714-L & 13.8 & 45.9 & 345 & $>1140$ & $>7940$ & 105 & 19308 & 36.8 \\
\hline DRLU081315-R & 11.1 & 201 & 41.6 & $>1140$ & 2384 & 58.5 & 37827 & $<35$ \\
\hline DRLU030815-R & 18.8 & 44.9 & 51.1 & $>1140$ & $>7940$ & 341 & 36977 & 75.6 \\
\hline DRLU121515-R & 8.9 & 32.9 & 28.6 & $>1140$ & 2465 & 18.8 & 39802 & $<35$ \\
\hline DRLU010416-R & 15.6 & 324 & $>1180$ & $>1140$ & 2033 & $>1920$ & 20708 & $<35$ \\
\hline DRLU112815-R & 25.7 & 248 & 136 & $>1140$ & $>7940$ & 402 & 25495 & 49.9 \\
\hline DRLU101615-R & $<8.9$ & 151 & 103 & $>1140$ & 235 & 108 & 48829 & $<35$ \\
\hline RLU1016151A-L & $<8.9$ & 408 & 60.0 & $>1140$ & $<98$ & $<7.9$ & 63135 & $<35$ \\
\hline 2013-678L & 12.0 & 328 & 47.1 & $>1140$ & $>7940$ & 131 & 37272 & 38.0 \\
\hline RLU0510162A-R & 13.8 & 50.3 & 180 & $>1140$ & 716 & 649 & 11472 & $<35$ \\
\hline RLU0728161A-L & $<8.9$ & 21.1 & 279 & 1077 & 1514 & 48.6 & 27263 & $<35$ \\
\hline RLU0722161B-L & 23.8 & 962 & $>1180$ & $>1140$ & $>7940$ & 473 & 25296 & 84.2 \\
\hline RLU0126161C-L & 9.3 & 52.0 & 218 & $>1140$ & 1660 & 238 & 5566 & $<35$ \\
\hline RLU0510161A-L & 16.0 & 84.6 & 135 & $>1140$ & 1739 & 361 & 16623 & $<35$ \\
\hline RLU0426161B-L & $<8.9$ & 16.9 & 62.9 & $>1140$ & 3055 & 93.7 & 27070 & $<35$ \\
\hline RLU0126162C-R & $<8.9$ & 31.0 & 238 & $>1140$ & 500 & 198 & 8362 & $<35$ \\
\hline RLU0720161B-L & 12.0 & 204 & 109 & $>1140$ & 3388 & 143 & 45793 & $<35$ \\
\hline RLU1028161B-L & 10.9 & 50.0 & 92.5 & $>1140$ & 3930 & 18.2 & 28538 & $<35$ \\
\hline RLU1115162B-R & $<8.9$ & $<16.3$ & 79.5 & $>1140$ & 773 & 191 & 13145 & $<35$ \\
\hline RLU0913162A-R & 9.3 & 71.5 & 862 & $>1140$ & $>7940$ & 27.7 & 29092 & 46.4 \\
\hline RLU0426162B-R & $<8.9$ & $<16.3$ & 37.3 & $>1140$ & 1333 & 116 & 28094 & $<35$ \\
\hline RLU0829162C-R & $<8.9$ & $<16.3$ & 70.1 & 623 & 567 & 38.1 & 16681 & $<35$ \\
\hline 16-04-765R & 10.7 & 310 & 132 & $>1140$ & 213 & 26.1 & 56552 & $<35$ \\
\hline 16-10-1103R & 14.3 & 372 & $>1180$ & $>1140$ & 598 & $>1920$ & 37681 & 39.8 \\
\hline RLU0204172A-R & 12.5 & 35.3 & 26.9 & $>1140$ & $>7940$ & 22.0 & 32826 & 53.6 \\
\hline RLU0317172B-R & 15.4 & 74.8 & $>1180$ & $>1140$ & 990 & 159 & 55165 & 37.9 \\
\hline RLU0319171A-L & 15.9 & 210 & 75.3 & $>1140$ & 1948 & 26.8 & 40339 & 42.1 \\
\hline RLU1121161A-L & 13.3 & 29.7 & $>1180$ & $>1140$ & 416 & 78.5 & 31780 & 46.7 \\
\hline RLU1121162A-R & 11.0 & 46.2 & 119 & $>1140$ & 1021 & 10.5 & 57380 & $<35$ \\
\hline RLU1125161A-L & $<8.9$ & $<16.3$ & 14.8 & $>1140$ & 195 & 33.9 & 25511 & $<35$ \\
\hline RLU1125162A-R & $<8.9$ & 23.0 & 28.7 & $>1140$ & 251 & 41.4 & 38353 & $<35$ \\
\hline Median & 12.9 & 73.2 & 114 & --- & 1739 & 108 & 30436 & --- \\
\hline IQR & 4.5 & 177 & 238 & --- & 5277 & 254 & 14566 & --- \\
\hline
\end{tabular}


Table 3-7. Periprosthetic tissue metal concentrations reported in $(\mu \mathrm{g} / \mathrm{L})$

\begin{tabular}{cccccc}
\hline Specimen & Cobalt & Chromium & Titanium & $\begin{array}{c}\text { Condyle } \\
\text { Material }\end{array}$ & $\begin{array}{c}\text { Tray } \\
\text { Material }\end{array}$ \\
\hline DRLU051714-L & 16.4 & 46.6 & 44.3 & CoCrMo & TiAlV \\
DRLU081315-R & 56.6 & 87.8 & 1136.3 & CoCrMo & TiAlV \\
DRLU030815-R & 14.8 & 37.3 & 37.1 & CoCrMo & TiAlV \\
DRLU121515-R & 4.2 & 301.6 & 50.9 & CoCrMo & TiAlV \\
DRLU010416-R & 0.4 & 7.4 & 4.4 & Oxinium & TiAlV \\
DRLU112815-R & 18.4 & 76.8 & 2.3 & CoCrMo & CoCrMo \\
DRLU101615-R & 49.1 & 134.7 & 3.2 & CoCrMo & CoCrMo \\
RLU0510162A-R & 1.6 & 23.5 & 46.8 & CoCrMo & TiAlV \\
RLU0728161A-L & 75.1 & 91.7 & 9.6 & CoCrMo & TiAlV \\
RLU0309161B-L & 3.5 & 7.4 & 3.8 & CoCrMo & TiAlV \\
RLU0722161B-L & 23.3 & 21.3 & 4.3 & CoCrMo & UHMWPE \\
RLU0126161C-L & 48.2 & 188.5 & 6.9 & CoCrMo & TiAlV \\
RLU0510161A-L & 1.8 & 13.4 & 51.6 & CoCrMo & TiAlV \\
RLU0426161B-L & 28705.3 & 66532.2 & 3.5 & CoCrMo & CoCrMo \\
RLU0126162C-R & 39.1 & 111.3 & 16.2 & CoCrMo & TiAlV \\
RLU1028161B-L & 0.7 & 23.4 & 12775.1 & CoCrMo & TiAlV \\
RLU1115162B-R & 146.4 & 38.3 & 28.9 & TiAlV & TiAlV \\
RLU0913162A-R & 1.3 & 5.1 & 98.7 & CoCrMo & TiAlV \\
RLU0426162B-R & 51.2 & 38.5 & 7.3 & CoCrMo & TiAlV \\
RLU0829162C-R & 39.0 & 154.1 & 8.8 & CoCrMo & CoCrMo \\
16-04-765R & 6.3 & 55.2 & 9.8 & CoCrMo & CoCrMo \\
16-10-1103R & 10.0 & 21.3 & 0.6 & CoCrMo & CoCrMo \\
16-10-1103L & 2.0 & 11.8 & 0.5 & CoCrMo & CoCrMo \\
Median & 16.4 & 38.5 & 9.6 & & \\
IQR & 45.9 & 80.2 & 41.5 & & \\
\hline
\end{tabular}




\section{Correlation of Study Parameters}

In the following sections, comparisons of the data sets were presented in graphical form. For internal/external rotational laxity and varus/valgus laxity, the data was presented as the sum of the absolute value of the two poles. For anterior and posterior laxity, the data was presented separately so that it was not skewed by the sample population containing both PS and CR implants. PS and CR implants were plotted with different symbols as indicated in the legend of each figure.

Because the data was not normally distributed, nonparametric Spearman Rank correlation analysis was performed. In Spearman rank correlation analysis, the ranks of each data point in the two data sets were calculated and then the difference in rank of data points between data sets was then used to compute the correlation coefficient. A coefficient of +1 indicated a perfect positive correlation and a coefficient of -1 indicated a perfect negative correlation. Spearman correlation coefficients $(\rho)$ with respective $p$ values (p) were included in tabular form. As previously mentioned, three specimens had extremely elevated $\mathrm{Ti}, \mathrm{Co}$, or Cr levels. These outliers were removed in the respective comparisons.

\section{Hypothesis (a) - Laxity and Tissue Metal Concentrations}

Graphical representation of the comparison between the joint laxity and tissue metal concentrations was presented in Appendix B, Figures B-1 through B-24. Spearman rank correlation analysis of the comparison between IE rotational laxity and tissue metal concentrations identified one significant relationship as reported in Table 3-8. Decreased IE laxity at full extension was inversely correlated with increased Co concentrations in the periprosthetic tissues $(\rho=-0.64, p=0.02)$. This comparison, shown in Figure B-1A, had a sample size of 13 and a 95\% confidence interval from -0.88 to -0.14 .

While spearman rank correlation analysis of VV laxity and tissue metal content did not reveal any statistically significant relationships, as shown in Table 3-9, VV laxity was moderately correlated with $\mathrm{Ti}$ in periprosthetic tissues at full extension and $30^{\circ}$ of flexion. At full extension, decreased VV laxity correlated with increased Ti content in the periprosthetic tissues (Figure B-11A, $\rho=-0.46, p=0.09$ ) with a sample size of 13 and a $95 \%$ confidence interval from -0.79 and 0.09 . Similarly, at $30^{\circ}$ of flexion, decreased VV laxity correlated with increased Ti concentrations (Figure B-11B, $\rho=-0.49, p=0.07$ ) with a sample size of 14 and a $95 \%$ confidence interval from -0.81 to 0.05 .

Spearman rank correlation analysis identified four significant relationships between AP laxity and tissue metal concentrations as reported in Table 3-10. At $90^{\circ}$ of flexion, anterior laxity was inversely correlated with Co concentrations (Figure B-14B, $\rho=-0.66, p=0.03$ ) with a sample size of 11 and a $95 \%$ confidence interval from -0.90 to 0.10 . At $60^{\circ}$ of flexion, anterior laxity was inversely correlated with $\mathrm{Cr}$ concentrations (Figure B-18A, $\rho=-0.63, p=0.03$ ) with a sample size of 13 and a $95 \%$ confidence interval from -0.88 to -0.12 . Anterior laxity at $90^{\circ}$ of flexion was also inversely correlated with $\mathrm{Cr}$ 
Table 3-8. Spearman correlation coefficients $(\rho)$ for IE rotational laxity and periprosthetic tissue metal concentrations

\begin{tabular}{ccccc}
\hline & \multicolumn{3}{c}{ Internal/External Rotational Laxity } \\
\cline { 2 - 5 } Metal & $\mathbf{0}^{\circ}$ & $\mathbf{3 0}^{\circ}$ & $\mathbf{6 0}^{\circ}$ & $\mathbf{9 0}^{\circ}$ \\
\hline Co & $-0.64^{*}$ & -0.07 & -0.01 & -0.21 \\
$\mathbf{C r}$ & -0.42 & 0.01 & -0.05 & -0.27 \\
$\mathbf{T i}$ & -0.15 & -0.28 & -0.06 & -0.07 \\
\hline
\end{tabular}

${ }^{*} \mathrm{p}=0.02$

Table 3-9. Spearman correlation coefficients $(\rho)$ for $V V$ laxity and periprosthetic tissue metal concentrations

\begin{tabular}{ccccc}
\hline & \multicolumn{4}{c}{ Varus/Valgus Laxity } \\
\cline { 2 - 5 } Metal & $\mathbf{0}^{\circ}$ & $\mathbf{3 0}^{\circ}$ & $\mathbf{6 0}^{\circ}$ & $\mathbf{9 0}^{\circ}$ \\
\hline Co & -0.22 & 0.09 & -0.13 & -0.27 \\
$\mathbf{C r}$ & -0.07 & 0.002 & 0.03 & -0.33 \\
$\mathbf{T i}$ & -0.46 & -0.49 & -0.11 & 0.02 \\
\hline
\end{tabular}

Table 3-10. Spearman correlation coefficients ( $\rho$ ) for AP laxity and periprosthetic tissue metal concentrations

\begin{tabular}{cccccccccc}
\hline & \multicolumn{4}{c}{ Anterior Laxity } & & \multicolumn{3}{c}{ Posterior Laxity } \\
\cline { 2 - 4 } \cline { 7 - 9 } Metal & $\mathbf{0}^{\circ}$ & $\mathbf{3 0}^{\circ}$ & $\mathbf{6 0}^{\circ}$ & $\mathbf{9 0}^{\circ}$ & & $\mathbf{0}^{\circ}$ & $\mathbf{3 0}^{\circ}$ & $\mathbf{6 0}^{\circ}$ & $\mathbf{9 0}^{\circ}$ \\
\hline Co & -0.50 & -0.22 & -0.45 & $-0.66^{* * *}$ & & -0.38 & -0.01 & -0.34 & -0.43 \\
Cr & -0.28 & -0.28 & $-0.63^{* * *}$ & $-0.81^{*}$ & & -0.52 & -0.41 & -0.50 & $-0.74^{* *}$ \\
Ti & 0.45 & 0.29 & 0.04 & 0.34 & & -0.20 & 0.20 & 0.13 & -0.02 \\
\hline
\end{tabular}

${ }^{*} \mathrm{p}=0.003$
${ }^{* *} \mathrm{p}=0.01$
${ }^{* *} \mathrm{p}=0.03$ 
concentrations (Figure B-18B, $\rho=-0.81, p=0.003$ ) with a sample size of 11 and a $95 \%$ confidence interval from -0.95 to -0.41 . Lastly, posterior laxity at $90^{\circ}$ of flexion was inversely correlated with $\mathrm{Cr}$ concentrations in the periprosthetic tissues (Figure B-20B, $\rho=-0.74, p=0.01)$ with a sample size of 11 and a $95 \%$ confidence interval from -0.93 to 0.25 .

\section{Hypothesis (b)_Laxity and Inflammatory Cytokines}

Graphical representations of each laxity and cytokine comparison was shown in Appendix C in Figures C-1 through C-48. Spearman rank correlation analysis revealed only one statistically significant correlation between IE rotational laxity and inflammatory cytokines in the joint synovial fluid, as shown in Table 3-11. At $60^{\circ}$ of flexion, IE rotational laxity was inversely correlated with IL-1 $\beta$ concentrations (Figure $\mathrm{C}$ $4 A, \rho=-0.55, p=0.02$ ) with a sample size of 18 and a $95 \%$ confidence interval from -0.81 to -0.11 .

As shown in Table 3-12, Spearman rank correlation analysis identified two statistically significant relationships between VV laxity and concentrations of inflammatory cytokines in the synovial fluid. At $60^{\circ}$ of flexion, VV laxity was inversely correlated with TNF- $\alpha$ concentrations (Figure $C-14 \mathrm{~A}, \rho=-0.78, \mathrm{p}=2 \times 10^{-4}$ ). This strong correlation had a sample size of 14 and a $95 \%$ confidence interval from -0.93 to -0.43 . However, it must be noted that levels of TNF- $\alpha$ were barely above the minimum detection limit; as such, this correlation was likely clinically insignificant. Additionally, VV laxity was inversely correlated with IL- $1 \beta$ concentrations (Figure C-16A, $\rho=-0.48$, $\mathrm{p}=0.04$ ) at $60^{\circ}$ of flexion with a sample size of 18 and a $95 \%$ confidence interval from 0.77 to -0.02 . At full extension, VV laxity was inversely correlated with IL-6 concentrations (Figure $\mathrm{C}-17 \mathrm{~A}, \rho=-0.46, \mathrm{p}=0.04$ ) with a sample size of 19 and a $95 \%$ confidence interval from -0.76 to -0.01 .

Spearman rank correlation analysis revealed two statistically significant relationships, as shown in Table 3-13. Anterior laxity was directly correlated with IL-6 concentrations (Figure $\mathrm{C}-34 \mathrm{~B}, \rho=0.53, \mathrm{p}=0.02$ ) at $90^{\circ}$ of flexion with a sample size of 18 and a $95 \%$ confidence interval from 0.08 to 0.80 . Additionally, anterior laxity was directly correlated with MCP- 1 concentrations (Figure C-38B, $\rho=0.62, p=0.006$ ) at $90^{\circ}$ of flexion with a sample size of 18 and a $95 \%$ confidence interval 0.22 to 0.84 . While not statistically significant, posterior laxity was directly correlated with IL- 6 at $30^{\circ}$ and $90^{\circ}$ of flexion. At $30^{\circ}$ of flexion, increased posterior laxity correlated with increased IL- 6 concentrations in the synovial fluid (Figure $\mathrm{C}-35 \mathrm{~B}, \rho=0.39, \mathrm{p}=0.09$ ) with a sample size of 20 and a $95 \%$ confidence interval from -0.06 to 0.71 . At $90^{\circ}$ of flexion, increased posterior laxity correlated with increased IL-6 concentrations in the synovial fluid (Figure $\mathrm{C}-36 \mathrm{~B}, \rho=0.46, \mathrm{p}=0.06$ ) with a sample size of 18 and a $95 \%$ confidence interval from 0.01 to 0.76 . 
Table 3-11. Spearman correlation coefficients ( $\rho)$ for IE laxity and inflammatory cytokine concentrations in the synovial fluid

\begin{tabular}{ccccc}
\hline & \multicolumn{4}{c}{ Internal/External Rotational Laxity } \\
\cline { 2 - 5 } Cytokine & $\mathbf{0}^{\circ}$ & $\mathbf{3 0}^{\circ}$ & $\mathbf{6 0}^{\circ}$ & $\mathbf{9 0}^{\circ}$ \\
\hline TNF- $\boldsymbol{\alpha}$ & -0.46 & -0.30 & -0.23 & 0.07 \\
IL-1 $\boldsymbol{\beta}$ & 0.42 & -0.14 & $-0.55^{*}$ & -0.33 \\
IL-6 & 0.16 & -0.16 & -0.24 & 0.13 \\
MCP-1 & 0.05 & -0.24 & -0.36 & 0.02 \\
MIP-3 $\boldsymbol{\alpha}$ & 0.17 & 0.27 & -0.17 & -0.19 \\
M-CSF & -0.16 & -0.30 & 0.04 & 0.04 \\
\hline
\end{tabular}

${ }^{*} \mathrm{p}=0.02$

Table 3-12. Spearman correlation coefficients $(\rho)$ for VV laxity and inflammatory cytokine concentrations in the synovial fluid

\begin{tabular}{ccccc}
\hline & \multicolumn{5}{c}{ Varus/Valgus Laxity } \\
\cline { 2 - 5 } Cytokine & $\mathbf{0}^{\circ}$ & $\mathbf{3 0}^{\circ}$ & $\mathbf{6 0}^{\circ}$ & $\mathbf{9 0}^{\circ}$ \\
\hline TNF- $\alpha$ & -0.19 & -0.41 & $-0.78^{*}$ & -0.18 \\
IL-1 $\beta$ & 0.30 & 0.11 & $-0.48^{* *}$ & -0.10 \\
IL-6 & $-0.46^{* *}$ & -0.16 & -0.05 & 0.07 \\
MCP-1 & -0.22 & -0.04 & -0.06 & 0.21 \\
MIP-3 $\alpha$ & -0.24 & -0.12 & -0.35 & -0.30 \\
M-CSF & -0.14 & 0.01 & 0.07 & 0.08 \\
\hline
\end{tabular}

${ }_{* *}^{*}=2 \times 10^{-4}$
$\mathrm{p}=0.04$ 
Table 3-13. Spearman correlation coefficients ( $\rho)$ for AP laxity and inflammatory cytokine concentrations in the synovial fluid

\begin{tabular}{|c|c|c|c|c|c|c|c|c|}
\hline \multirow[b]{2}{*}{ Cytokine } & \multicolumn{4}{|c|}{ Anterior Laxity } & \multicolumn{4}{|c|}{ Posterior Laxity } \\
\hline & $\mathbf{0}^{\circ}$ & $30^{\circ}$ & $60^{\circ}$ & $90^{\circ}$ & $\mathbf{0}^{\circ}$ & $30^{\circ}$ & $60^{\circ}$ & $90^{\circ}$ \\
\hline TNF- $\alpha$ & 0.11 & -0.16 & 0.04 & 0.03 & -0.39 & -0.03 & -0.24 & 0.22 \\
\hline IL-1 $\beta$ & 0.40 & -0.14 & 0.03 & -0.06 & -0.31 & 0.22 & -0.14 & -0.19 \\
\hline IL-6 & 0.36 & -0.03 & 0.30 & $0.53^{* *}$ & 0.02 & 0.39 & -0.10 & 0.46 \\
\hline MCP-1 & 0.32 & 0.13 & 0.00 & $0.62^{*}$ & -0.23 & -0.02 & 0.20 & 0.38 \\
\hline MIP-3 $\alpha$ & -0.11 & 0.10 & 0.08 & 0.14 & -0.30 & 0.01 & -0.30 & 0.16 \\
\hline M-CSF & 0.05 & -0.09 & 0.10 & 0.01 & 0.12 & 0.12 & 0.14 & -0.08 \\
\hline
\end{tabular}

${ }_{* *}^{*}=0.006$
$\mathrm{p}=0.02$ 


\section{Hypothesis (c)_-Tissue Metal Concentrations and Inflammatory Cytokines}

Graphical representation of the comparison between inflammatory cytokines and tissue metal concentrations was presented in Appendix D in Figures D-1 through D-18. Spearman rank correlation analysis with outliers removed identified only two statistically significant relationships between tissue metal concentrations and inflammatory cytokines as reported in Table 3-14. As shown in Figure D-9, Cr was inversely correlated with IL-6 $(\rho=-0.52, p=0.01)$ with a sample size of 21 and a $95 \%$ confidence interval from -0.78 to 0.11 . Cr was also inversely correlated with MIP-3 $\alpha$ (Figure D-11, $\rho=-0.46, p=0.04$ ) with a sample size of 21 and a $95 \%$ confidence interval from -0.74 to -0.04 . While not statistically significant, Spearman rank correlation analysis also revealed four other inverse correlations. In Figure D-8, periprosthetic tissue Cr content was inversely correlated with IL-1 $\beta$ concentrations in the synovial fluid $(\rho=-0.41, p=0.09)$ with a sample size of 18 and a $95 \%$ confidence interval from -0.74 to 0.07 . The measured $\mathrm{Cr}$ concentrations was also inversely correlated with MCP-1 concentrations (Figure D-10, $\rho=-0.40, p=0.07$ ) with a sample size of 21 and a $95 \%$ confidence interval from -0.71 and 0.04 . Increased Ti concentrations was correlated with decreased IL- $1 \beta$ concentrations (Figure D-14, $\rho=-0.41, p=0.09$ ) with a sample size of 17 and a 95\% confidence interval from -0.74 to -0.09 . Lastly, Ti was inversely correlated with MIP-3 $\alpha$ concentrations in the synovial fluid (Figure D-17, $\rho=-0.40, p=0.08$ ) with a sample size of 20 and a $95 \%$ confidence interval from -0.71 to 0.05 .

Table 3-14. Spearman correlation coefficients $(\rho)$ for tissue metal concentrations and inflammatory cytokine concentrations in the synovial fluid with outliers removed

\begin{tabular}{cccc}
\hline Cytokine & Co & Cr & Ti \\
\hline TNF- $\alpha$ & 0.19 & -0.23 & -0.44 \\
IL-1 $\beta$ & -0.11 & -0.41 & -0.41 \\
IL-6 & -0.32 & $-0.52^{*}$ & -0.22 \\
MCP-1 & -0.31 & -0.40 & 0.08 \\
MIP-3 $\boldsymbol{\alpha}$ & -0.14 & $-0.46^{* *}$ & -0.40 \\
M-CSF & -0.10 & -0.02 & -0.21 \\
\hline
\end{tabular}

${ }_{* *}^{*}=0.01$
$\mathrm{p}=0.04$ 


\section{CHAPTER 4. DISCUSSION}

The specimens included in this study consisted of primary TKA implants that were retrieved at necropsy and were determined to be well-fixed per fluoroscopic analysis. The first objective of this study was to establish a baseline of different factors that likely contribute to failure of primary total knee replacements. This study provided semi-quantitative assessment of wear of the condylar surface, backside surface, and stabilizing post, when applicable, and of damage to the bearing surface of the femoral components. Joint laxity was measured using a custom knee testing platform that had been previously studied and utilized by published articles and theses in literature (114, 184-193). Inflammatory cytokine profiles in the synovial fluid samples were presented and measurable levels of metal debris was found in the periprosthetic tissue samples. The second objective of this study was to determine if any significant relationships arose in correlation analysis of the latter three aforementioned factors. An inverse trend was observed between joint laxity and tissue metal concentrations, such that decreased laxity induced metal release from the implant components. A direct trend was observed specifically between increased anteroposterior laxity and elevated inflammatory cytokines. Lastly, an inverse trend was found between decreased tissue metal concentrations and increased inflammatory cytokines. The third, and future, objective of this study is to utilize these measurements and observations for comparison with failed implants retrieved at time of revision.

\section{Implant Wear and Damage Assessment}

Wear in TKA is inevitable. While the current knowledge of the mechanisms involved in debris-induced TKA failure is still in its infancy, it is believed that excessive polyethylene wear particles stimulate a cascade of biological events that lead to degradation of bone and inhibition of bone formation (131, 132, 137, 166, 169, 171, 202, 203). This wear and biologic response pathway is termed osteolysis, and oftentimes necessitates revision of the index procedure. Orthopedic implant retrieval programs are of utmost importance in expanding the understanding of the etiology of aseptic implant loosening. While most retrieval programs analyze failed implants retrieved at time of revision, the implants in this study were retrieved at necropsy and were deemed to be well-functioning primary TKAs based on fluoroscopic analysis. In order to assess how soft tissue laxity, periprosthetic tissue metal concentrations, and inflammatory cytokines concentrations in the synovial fluid contribute to the interaction between human physiology and the implant, the degree of wear and damage to the implant systems themselves first were established.

The modes of wear can be grouped by the following mechanisms: adhesive wear, abrasive wear, and fatigue wear. Characteristic of adhesive or abrasive wear, burnishing of the condylar, backside, and post surfaces may result from cyclic loading and micromotion which leads to progressive buffing of the original matte surface finish. As in many other retrieval studies, burnishing was the most common wear mode on every 
assessed surface (32). From a biological perspective, burnishing produces wear debris that is within the size range that may stimulate an inflammatory response. Pitting, a mechanism of fatigue wear, is sometimes referred to as "cratering" and involves the liberation of millimeter-sized pieces of wear debris from the articulating surface. The wear debris produced by pitting is considered to be too large to provoke an osteolytic response; as such, pitting is believed to be a more benign wear mechanism than adhesive or abrasive wear, which produces micrometer-sized debris (204). Scratching is a mode of abrasive wear produced by plowing of microscopic asperities on the opposing metallic surface or by third body debris (204). Pitting and scratching were common wear mechanisms observed on all insert surfaces. Burnishing, pitting, and scratching were the predominant wear modes for all surfaces assessed.

Tibial insert wear on the condylar surface, backside surface, and stabilizing post, when applicable, was assessed using a modified Hood et al. (195) method. While the predominant modes of wear included burnishing, pitting, and scratching for all surfaces assessed, there were 2 implants with severe delamination on the condylar surface and 11 implants with mild to severe backside surface deformation. Specimen DRLU081315-R, a posterior-stabilized, fixed bearing tibial insert sterilized with gamma radiation in an inert gas environment, had the highest condylar wear with a total score of 64. Severe burnishing was identified on the condylar and backside surfaces, with the maximum possible score of 24. Additionally, this implant had the highest wear of the stabilizing post with a total score of 22; burnishing was the predominant mode of wear (score of 10 out of 12) followed by abrasion (score of 5 out of 12). Specimen RLU0913162A-R, a posterior-stabilized, fixed bearing tibial insert sterilized with gamma radiation in air, demonstrated the second highest condylar wear with a total score of 58, moderate backside wear with a total score of 56, and the second highest wear of the stabilizing post with a total score of 16. Specimen RLU1028161B-L, a fixed bearing tibial insert sterilized with gamma radiation in air, had a total condylar wear score of 57 with severe condylar delamination (score of 22 out of 24) accompanied by oxidation. This implant had the highest backside wear with a total score of 84; the modes of burnishing, pitting, and scratching each had the maximum score of 24 , while the mode of surface deformation had a score of 12 .

Many factors contribute to wear of the tibial insert on all aforementioned surfaces, including but not limited to: patient-specific factors such as body mass index and duration of implantation (195, 205-207); surgical-related factors such as ligament balancing or alignment of the components $(78,123-125,208,209)$; and lastly implantspecific factors such as method of fabrication, mode of sterilization, and design (210212). In this study, duration of implantation was not a statistically significant factor $(p>0.05)$ in the degree of wear. However, this is likely due to the fact that these were all well-functioning specimens retrieved at necropsy instead of revision. The contribution of ligament balancing will be discussed further in context of measured laxity in the following sections. The mode of fabrication was not available for the implants and thus was not considered in this study, but the mode of sterilization was available for the implants. 
Gamma irradiation in air was the method utilized by most to sterilize UHMWPE for use in both total hip and total knee arthroplasty components prior to 2000 (204). However, oxidative degradation of the polyethylene components occurred following gamma radiation sterilization in air and prolonged shelf storage in air $(213,214)$. Additionally, oxidation during long-term shelf aging following gamma irradiation was discovered to cause a loss in mechanical properties and in some cases, more rapid polyethylene wear often through delamination, especially with respect to TKA $(205,211$, $215,216)$. Repeated cyclic loading of the UHMWPE, which results in subsurface fatigue and failure of the UHMWPE tibial insert, causes delamination wear $(216,217)$. Delamination is often associated with production of large amounts of debris and gross component failure. To address the concern of oxidation in gamma-air sterilized implants, orthopaedic manufacturers in the mid-to-late 1990s discontinued gamma sterilization in air and introduced gamma sterilization in low-oxygen or inert gas environment in combination with barrier packaging to reduce risk of oxidation prior to implantation (204). Although many biomechanical and material factors induce and accelerate delamination failure, stabilization of UHMWPE through sterilization without irradiation or irradiation sterilization in an inert atmosphere is thought to eliminate subsurface oxidation which contributes to delamination. Specimen RLU1028161B-L, in Figure 3-2, illustrates the detrimental effects of oxidation caused by gamma-air sterilization.

The contemporary TKA tibial component can either be a fixed or mobile bearing design. Mobile bearing designs were introduced to provide congruity of the tibiofemoral articular surface and to allow relative movement between the lower surface of the polyethylene insert and the metal tibial baseplate for reduction of the constraint force (81, $82,218)$. Because the contact forces are theoretically reduced on the articular surface mobile bearing knees, the risk of polyethylene wear may also be reduced. However, the articulation between the inferior surface of the tibial insert and the superior surface of the tibial tray provides an additional source of wear debris generation. Lu et al. (218) found that while the polyethylene wear on the superior surface was in fact reduced with the mobile bearing design, there was a higher incidence of high-grade wear patterns, such as scratching, pitting, embedded debris, and delamination (218). A student's t-test revealed that the differences in condylar and backside wear scores between mobile bearing and fixed bearing implants in this cohort were not statistically significant ( $p>0.05)$. Moreover, the incidence of "high-grade" wear patterns on the backside surface, as mentioned in the study by Lu et al. (218) was not statistically significant with respect to fixed bearing versus mobile bearing designs. Again, this is likely due to the fact that the implants in this cohort are well-functioning implants.

Although the articulating surface of polyethylene insert was thought to be the main source of debris generated in total knee arthroplasty, the tibial tray-insert interface has also been established as a contributing source of polyethylene debris $(89,206,208$, $210,219)$ and in many cases has been related to tibial osteolysis $(89,220,221)$. Factors influencing backside interface micromotion and the resultant wear include: implant design, tibial tray material (CoCrMo or TiAlV), locking mechanism design in fixed bearing tibial components, and surface roughness $(222,223)$. Previous studies have indicated that the backside surface of a fixed bearing tibial insert can still move relative 
to the tray despite being secured to the tibial tray with a locking mechanism $(206,224$, 225). This present study gives further evidence of micromotion occurring between fixed bearing tibial insert-tray designs.

While burnishing was the predominant mode of wear on the backside surface, pitting and scratching were also observed. Surace et al. (210) proposed that pitting on the backside surface may occur by a different mechanism than that on the condylar surface because contact-induced stresses near the backside surface of the insert are much smaller than those observed on the condylar surface. Instead, the formation of pits or scratches may be due to the presence of third-body particles, such as Ti particles, produced by micromotion at the interface $(78,125,126)$. Additionally, surface deformation on the backside surface was observed on 11 out of 25 of the inserts. Surface deformation, oftentimes referred to as cold flow in literature, was characterized by cylindrical protrusions of polyethylene extending from the undersurface of the insert into screwholes within the tibial base-plate. This method of wear has been well-documented in literature and has been attributed to cold flow or creep of the polyethylene under the action of compressive forces applied to the upper surface of the insert during weightbearing activities $(89,206,210,219)$. However, the results of a study by Conditt et al. (206) suggested that the protrusions on the underside of worn retrieved tibial components were not primarily due to creep or cold flow of the polyethylene through the screw-holes on the baseplate, but instead were primarily due to wear of the inserts. They found that the surfaces of all of the polyethylene material descending below the worn backside surface existed in a single plane, independent of the location of each individual cylinder, an observation unlikely to occur if the protrusions were due to cold flow or creep (206).

Another design element that contributes to the polyethylene debris burden is the presence of a stabilizing post in PCL-substituting designs. While PCL-substituting designs were developed to prevent uncontrolled sliding of the femoral component without requiring excessive conformity, thereby reducing wear of the condylar insert surface, several studies have reported that the cam-post articulation in PS implants is an additional source of polyethylene wear debris $(32,109,205,226,227)$. Burnishing, scratching, pitting, and abrasion were the most prevalent wear modes in the current study. As shown in Figure 3-3, specimen RLU1121162A-R had significant damage to the anterior surface of the stabilizing post with little wear on the posterior surface. Wear on the posterior surface of the stabilizing post is expected as that is the surface where articulation occurs with the cam. However, many of the implants in this study exhibited wear of the anterior surface as well. Anterior impingement damage may occur on both PS and CR designs. The incidence of anterior impingement damage in PS designs has been reported to range from $36 \%$ to $75 \%$ in literature $(109,228)$. Banks et al. (228) suggested that as the femoral component moves from flexion to extension to hyperextension, the intercondylar notch contacts the polyethylene tibial eminence or post causing damage. The wear caused by anterior impingement could compromise the structural integrity of the stabilizing post leading to fracture and also likely generates micrometer-sized wear which may lead to stimulation of inflammatory processes. 
An important limitation of the Hood et al. (195) approach to assessing wear is that a high damage score does not necessarily indicate that a large amount of wear has occurred. Instead, this method only expresses damage in terms of the area covered by the defect. While thickness measurements can account for some loss of material, original implant thickness must be known and the irregular shape of a tibial insert likely would skew the measurements. Moreover, Puloski et al. (109) noted that discerning between mechanisms of wear in which material is lost and plastic deformation in which polymer is distorted without loss of material also poses another complication in quantifying or qualifying tibial insert wear.

Total knee arthroplasty femoral components present large metal surface areas that can undergo material loss due to wear and/or corrosion, generating metal particles and metal ions. While, historically, polyethylene debris was believed to be the primary cause of osteolysis and aseptic loosening, recent attention has been directed towards the role of metal debris in adverse events following primary total joint replacements. Possible corrosive mechanisms of metal debris production in primary TKAs include: direct inflammatory cell-induced corrosion $(145,146,196)$ and degradation at the backside interface with the bone cement layer in the form of discoloration (196). Possible abrasive wear mechanisms included scratching due to third-body debris $(196,229,230)$ and metalon-metal articulation due to complete wear-through of the polyethylene tibial insert or patellar button, in cases of metal-backed patellar resurfacing. In addition to generating debris that may incite inflammatory reactions, femoral component surfaces roughened by abrasive mechanisms have been shown to increase wear of the tibial insert (229-231). As such, wear of the femoral component may induce additional wear of the polyethylene insert.

Only one study was found in literature that analyzed damage of femoral components. Arnholt et al. (196) analyzed the aforementioned corrosive and abrasive wear mechanisms on $52 \mathrm{CoCr}$ femoral components retrieved at time of revision. They found evidence of metal release of corrosive damage in 51 of the 52 (98\%) femoral components included in their study with $98 \%(n=51 / 52)$ of the implants demonstrating third-body wear, primarily in the form of scratching. Such a high incidence of damage highlights the critical need for additional research that considers the role of femoral component damage in the failure of TKA. Using the same method employed by Arnholt et al. (196), the present study analyzed the damage in four quadrants of 29 wellfunctioning femoral components retrieved at autopsy. While the median damage score of the sample population was a score of 5 , mild to severe damage (score $\geq 2$ ) was observed in at least one quadrant of $70 \%$ of the femoral condyles included in this study. Two implants in particular displayed significant abrasive damage of the femoral component. Specimen RLU1028161B-L, shown in Figure 3-5, had severe scratching of the bearing surface and received a score of 14 out of the maximum score of 16 . The specimen was a Miller-Galante II design (Zimmer Inc.; Warsaw, IN) with a TiAlV femoral component. The lower modulus of elasticity of TiAlV with respect to CoCrMo makes it more prone to damage and wear. As such, TiAlV is not a suitable bearing material in total knee arthroplasty. This specimen also had significant condylar and backside wear of the tibial insert, which is in accordance with previous reports (229-231) of roughened femoral 
components increasing wear of the tibial insert. Specimen RLU0426161B-L, a press-fit, LCS Mobile Bearing design (Depuy; Warsaw, IN) with a condylar coating of CoCr small beads, had moderate scratching of the bearing surface, receiving a score of 10 . This specimen, shown in Figure 1-5, presented with metallosis which is associated with abnormal metallic wear $(151,152)$. The corresponding tibial insert condylar surface for specimen RLU0426161B-L demonstrated severe burnishing (22 out of 24), mild scratching and pitting (score 7 out of 24), mild delamination (score of 4), and some evidence of embedded metallic debris (score of 2).

\section{Tissue Metal Concentrations}

While the femoral component damage scores qualitatively described the wear that occurred in vivo, ICP-MS analysis quantitatively measured the metallic debris that had accumulated in the periprosthetic tissues. Kretzer et al. (232) was the first to question whether wear debris-induced failure in TKA was solely due to polyethylene. Seeking to quantify the extent of metal debris generation in TKA, they employed a wear simulator with all polymer bearings allowing the resultant metallic wear to be solely from the CoCrMo implants. While wear of metallic components has traditionally been attributed to third body particles such as bone chips or bone cement, Kretzer et al. (232) observed wear of the metal components without the presence of these third bodies in their simulation. Despite the absence of these "traditional" third body particles, the metallic femoral components displayed pitting as well as third body and abrasive wear mainly originating from the cobalt-rich matrix. They postulated that the third bodies inducing the pitting or abrasive wear were carbides released from the bulk alloy. Carbides are known to be very hard and rich in Mo and $\mathrm{Cr}$ (233). Consequently, the higher hardness of the carbides or fragments within the articulation may have caused the observed abrasive and third body wear of the femoral components (232). Additionally, Kretzer et al. (232) noted the release of titanium from the tibial plateau, although that tibial plateau is not intended for articulation. However, TiAlV alloy has a very thin oxide layer that is sensitive to fretting (234). Kretzer et al. (232) stated that, even with softer material like polyethylene which has a lower elastic modulus than TiAlV, micromotions may cause a relevant release of Ti wear products. Previous wear simulator studies have also reported evidence of large pits where particles had been removed from the superior surface of the TiAlV baseplate $(78,123,125,126)$. One shortcoming of wear simulator studies is that they typically measure debris release over five million cycles which translate to an implant lifespan of approximately five years.

While there is a plethora of studies that have investigated metal release in metalon-metal THAs, only five previous studies were found that reported metal ion concentrations in serum $(157,235)$ and metal concentrations in synovial fluid $(236,237)$ of patients with TKA. For sake of comparison to the present study, the reported values were converted to $\mu \mathrm{g} / \mathrm{L}$, as presented in Table 4-1. Three studies were identified that measured metal release from TKA in serum. Jacobs et al. (238) measured Ti release from cementless TiAlV alloy total knee replacements in three cohorts: control patients without implants $(n=21)$, well-functioning, unilateral primary TKA patients $(n=10)$, and patients 
Table 4-1. Compilation of previous studies investigation metal concentrations from TKA for comparison for current study

\begin{tabular}{lcccccc}
\hline Study & $\begin{array}{c}\text { Sample } \\
\text { Population }\end{array}$ & $\begin{array}{c}\mathbf{C o} \\
(\mu \mathbf{g} / \mathbf{L})\end{array}$ & $\begin{array}{c}\mathbf{C r} \\
(\mu \mathbf{g} / \mathbf{L})\end{array}$ & $\begin{array}{c}\mathbf{T i} \\
(\mu \mathbf{g} / \mathbf{L})\end{array}$ & $\begin{array}{c}\text { Sample } \\
\text { Medium }\end{array}$ & $\begin{array}{c}\text { Implant } \\
\text { Materials }\end{array}$ \\
\hline Jacobs et al. & $\begin{array}{c}\text { Control } \\
\text { Stable Unilateral }\end{array}$ & -- & -- & 2.67 & Serum & TiAlV \\
& Failed Unilateral & -- & -- & 3.15 & & \\
Luetzner et al. & Control & 0.25 & $<0.25$ & -- & Serum & CoCrMo \\
& Stable Unilateral & 3.27 & 0.92 & -- & & \\
Savarino et al. & Stable Bilateral & 4.28 & 0.98 & -- & & \\
& Control & 0.26 & 0.24 & 3.20 & Serum & CoCrMo \\
& Stable Unilateral & 0.44 & 0.24 & 2.91 & & and TiAlV \\
Cracchiolo et al. & Failed Unilateral & 1.10 & 0.45 & 3.05 & & \\
& Control & 800 & 0 & -- & Synovial & Unknown \\
& Synovitis Hinged & 6,540 & 36,240 & -- & Fluid & \\
& Synovitis & 1,230 & 1,930 & -- & & \\
Milosev et al. & Non-hinged & & & & & \\
& Control & $<0.2$ & $<0.02$ & 1.92 & Synovial & CoCrMo \\
Present Study & Failed & 1.13 & 5.92 & 2.20 & Fluid & and TiAlV \\
& Stable Necropsy & 16.36 & 38.54 & 9.62 & Tissue & CoCrMo \\
& & & & & & and TiAlV \\
\hline
\end{tabular}

Sources:

Jacobs JJ, Silverton C, Hallab NJ, Skipor AK, Patterson L, Black J, et al. Metal release and excretion from cementless titanium alloy total knee replacements. Clinical orthopaedics and related research. 1999(358):173-80.

Luetzner J, Krummenauer F, Lengel AM, Ziegler J, Witzleb W-C. Serum metal ion exposure after total knee arthroplasty. Clinical orthopaedics and related research. 2007;461:136-42.

Savarino L, Tigani D, Greco M, Baldini N, Giunti A. The potential role of metal ion release as a marker of loosening in patients with total knee replacement. Bone Joint J. 2010;92(5):634-8.

Cracchiolo A, Revell P. Metal concentration in synovial fluids of patients with prosthetic knee arthroplasty. Clinical orthopaedics and related research. 1982(170):169-74.

Milošev I, Levašič V, Vidmar J, Kovač S, Trebše R. $\mathrm{pH}$ and metal concentration of synovial fluid of osteoarthritic joints and joints with metal replacements. Journal of Biomedical Materials Research Part B: Applied Biomaterials. 2017;105(8):2507-15. 
with failed porous Ti coated patellar components $(n=8)$. The Ti concentrations measured in the failed cohort was statistically greater than the control cohort $(\mathrm{p}<0.005)$ while the Ti concentrations measured in the stable cohort were not statistically different from the control cohort. A study by Luetzner et al. (235), using atomic absorption spectrometry, reported median Co serum concentrations in patients with well-functioning unilateral $(n=18)$ and bilateral $(n=23)$ TKAs. They compared these Co and $\mathrm{Cr}$ concentrations to a control group from a previous study by Witzleb et al. (239). While no differences between serum levels in patients with unilateral and bilateral arthroplasties were observed, the researchers reported elevated serum levels of $\mathrm{Cr}$ and $\mathrm{Co}$ in both study groups when compared to the control group (235). A cohort study performed by Savarino et al. (157) investigated the role of ion release in the assessment of fixation of the implant after unilateral TKA. Their study compared serum levels of $\mathrm{Ti}, \mathrm{Cr}$, and $\mathrm{Co}$, measured by atomic absorption spectrometry, for three sample groups: no implants, stable implants, and failed implants. Evaluation of Ti was underestimated because of the high instrumental detection limit employed in the study. Based on the high specificity and positive predictive value, Savarino et al. (157) concluded that elevated $\mathrm{Cr}$ ion levels appears to be a good diagnostic indicator of loosening when clinical and radiological signs are unclear.

Two studies were identified that measured metal release from TKA in synovial fluid. Using atomic absorption spectrometry, a study by Cracchiolo et al. (236) measured the $\mathrm{Co}$ and $\mathrm{Cr}$ concentrations in the synovial fluid of patients with primary arthritis, patients with hinged (metal-on-metal) TKA prostheses, and patients with non-hinged (metal-on-polyethylene) TKA prostheses. Additionally, Cracchiolo et al. (236) measured the $\mathrm{Co}$ and $\mathrm{Cr}$ concentrations in the sera of only the control patients and patients with hinged prostheses. Interestingly, the only significant difference they observed was that the control patients had higher Co sera concentrations than the patients with hinged prostheses. As such, using serum as the medium for testing metal concentrations may underestimate the actual degree of metal debris accumulating in the joint. While measuring metal ion levels in serum may suit clinical detection of excessive wear, measuring metal debris in the synovial fluid or periprosthetic tissues is likely to be more indicative of how much debris has accumulated in the local periarticular environment. Milosev et al. (237) measured the $\mathrm{Co}, \mathrm{Cr}$, and $\mathrm{Ti}$ concentrations using inductivelycoupled mass spectrometry in the synovial fluid of a patients with primary OA collected at time of index TKA $(n=2)$ and patients with failed primary knee arthroplasty collected at time of revision $(\mathrm{n}=11)$. Cobalt and chromium were not detected in the control group but were detected in $82 \%(n=9 / 11)$ and $45 \%(n=5 / 11)$ of the failed cohort. When measuring metal concentrations in synovial fluid, the authors $(236,237)$ were unable to identify whether the debris was in ionic or particle form; the same concept will be applied to the present study.

To the author's knowledge, this is the first study to document $\mathrm{Co}, \mathrm{Cr}$, and $\mathrm{Ti}$ concentrations in the periprosthetic tissues of well-functioning TKA specimens retrieved at autopsy. Despite Co being the predominant element in the composition of CoCrMo alloys, the median Co concentration found in the periprosthetic tissues was $16.4 \mu \mathrm{g} / \mathrm{L}$, whereas the median $\mathrm{Cr}$ concentration was $38.5 \mu \mathrm{g} / \mathrm{L}$. Similarly, Ichinose et al. (240) 
examined the metal composition in the synovial tissues surrounding failed TKAs revised due to unexplained pain and swelling. Using backscattered scanning electron microscopy, they found that the ratio of $\mathrm{Co}, \mathrm{Cr}$ and Mo was 4.16:69.15:20.67, respectively, in the synovial cells of patients with CoCrMo TKA components. The authors speculated that Co may dissolve from periarticular cells, disseminating throughout the body, whereas $\mathrm{Cr}$ remains within the cells (240). Similarly, Woodman et al. (241) and Merritt et al. (242) indicated that Co dissolved into the fluid, ultimately removed from the body in urine, although $\mathrm{Cr}$ remained in the tissues around CoCrMo components. Taking this into consideration, the Co concentrations in this study may potentially underestimate the actual amount of Co released. Despite this potential limitation, this study presents a novel finding of elevated $\mathrm{Co}, \mathrm{Cr}$, and $\mathrm{Ti}$ concentrations in the periprosthetic tissues of wellfunctioning TKA specimens.

In the present study, three specimens had notably elevated $\mathrm{Co}, \mathrm{Cr}$, or $\mathrm{Ti}$ concentrations and were excluded from statistical analysis. Specimen DRLU081315-R had a Ti concentration of $1,136.3 \mu \mathrm{g} / \mathrm{L}$ in the periprosthetic tissues. This implant system was a cemented CoCrMo alloy femoral component with TiAlV tibial baseplate. A fixed bearing implant system, this specimen demonstrated significant backside wear of the tibial insert (scratching score of 24 out of 24, pitting score of 10 out of 24, burnishing score of 24 out of 24 , and surface deformation score of 8 out of 24) indicating micromotion occurred. The micromotion may have contributed to the wear of the TiAlV tibial baseplate. Specimen RLU0426161B-L had extremely high Co and Cr concentrations - 28,705 $\mu \mathrm{g} / \mathrm{L}$ and $66,532 \mu \mathrm{g} / \mathrm{L}$, respectively. As previously stated, this implant also endured significant damage in vivo, receiving a femoral component damage score of 10 and presented with severe metallosis, as shown in Figure 1-5. Additionally, this implant system was uncemented with a porous coating of small beads on the femoral condyle and tibial baseplate; however, the material composition of the coating is unknown. The $\mathrm{Co}$ and $\mathrm{Cr}$ concentrations observed in this specimen were greater than the mean $\mathrm{Co}$ and $\mathrm{Cr}$ concentrations measured in the synovial fluid of the patients with both non-hinged and hinged prostheses (Table 4-1) that had suspected synovitis, which were the highest metal concentrations reported in the included literature. Specimen RLU1028161B-L, a cementless, Ti fiber mesh coated TiAlV alloy femoral component and tibial baseplate, had extremely elevated Ti concentrations $(12775 \mu \mathrm{g} / \mathrm{L})$ in the periprosthetic tissues. This implant received the highest femoral component damage score (14 out of 16), the third highest wear score of the tibial insert condylar surface (57 out of 168) with notable delamination present (score of 22 out of 24), and the highest wear score of the tibial insert backside surface ( 84 out of 168). While each of these outliers have periprosthetic tissue concentrations that exceed the $\mathrm{Co}, \mathrm{Cr}$, and $\mathrm{Ti}$ concentrations measured in the sera and synovial fluid of stable and failed implants in previous studies, the median values reported in this study, which analyzed only wellfunctioning implants retrieved at autopsy, were higher than the average values reported in both the sera or synovial fluid. 


\section{Inflammatory Cytokines}

Through normal use of prosthetic joints, polyethylene and metallic wear particles are generated, as documented through the wear analysis of implants and measurements of tissue metal concentrations. These wear particles evoke an inflammatory response through the stimulation of macrophages and other immune cells prompting the release of powerful chemical mediators, which in turn may stimulate osteolysis. Inflammatory cytokines have been proposed as possible biomarkers for detection of TKA failure (177, $203,243,244)$. To establish the role of certain chemical mediators in the present study's cohort of well-functioning primary TKA retrieved at necropsy, a cytokine assay was performed for the detection of multiple analytes (TNF- $\alpha$, IL-1 $\beta$, IL-6, MCP-1, MIP-3 $\alpha$, and M-CSF) in the synovial fluid samples. However, to elucidate which the significance of the cytokine concentration measurements, the concentrations of these inflammatory mediators must first be established in those without prosthetic implants to serve as a standard for comparison. Several studies were identified that measured the concentrations of TNF- $\alpha$, IL-1 $\beta$, IL-6, MCP-1, MIP-3 $\alpha$, and M-CSF in the synovial fluid of patients diagnosed with OA or RA of the knee. While these cytokines may also play a role in the inflammatory processes in these conditions and are likely to elevated, these reported values will serve as a lower limit guideline for comparison. Additionally, a few studies were identified that measured the concentrations of the same cytokines in patients with aseptically failed total knee implants. These reported values will serve as an upper limit guideline for comparison.

Kahle et al. (245) measured the levels of TNF- $\alpha$ and IL-1 $\beta$ in the synovial fluid of patients with OA, patients with RA, and healthy control patients at necropsy. The researchers reported the following mean TNF- $\alpha$ concentrations in the OA, RA, and control cohorts: $80 \mathrm{pg} / \mathrm{mL}, 170 \mathrm{pg} / \mathrm{mL}$, and $289 \mathrm{pg} / \mathrm{mL}$. Additionally, they reported the following mean IL-1 $\beta$ concentrations in the OA, RA, and control cohorts: $21 \mathrm{pg} / \mathrm{mL}, 193$ $\mathrm{pg} / \mathrm{mL}$, and $<20 \mathrm{pg} / \mathrm{mL}$ (245). This particular study was novel as it was the only one found that presented the levels of these two inflammatory cytokines in the synovial fluid of healthy controls at autopsy. Mabey et al. (243) sought to quantify the concentrations of proinflammatory and anti-inflammatory cytokines in plasma and synovial fluid of primary knee OA patients $(n=32)$ and healthy controls. As is the case with most studies, plasma was the only type of sample collected from the healthy controls for analysis. Due to this ethical constraint, it is difficult to discern the naturally occurring concentrations of the cytokines of interest. However, for reference, Mabey et al. (243) reported the following average cytokine concentrations in the cohort of OA patients-TNF- $\alpha$ : 1.0 $\mathrm{pg} / \mathrm{mL}$ (lower detection limit: $0.20 \mathrm{pg} / \mathrm{mL}$ ), IL-1 $\beta$ : $1.9 \mathrm{pg} / \mathrm{mL}$ (lower detection limit: 0.10 $\mathrm{pg} / \mathrm{mL}$ ), and IL-6: $356.5 \mathrm{pg} / \mathrm{mL}$ (lower detection limit: $0.29 \mathrm{pg} / \mathrm{mL}$ ). Interestingly, Mabey et al. found that TNF- $\alpha$ and IL-1 $\beta$ in OA plasma were significantly higher than in paired synovial fluid samples $(\mathrm{p}<0.001)$. On the other hand, Steiner et al. (246) measured TNF-

$\alpha$ and IL-6 in the synovial fluid from patients with RA $(n=33)$ and reported the following average cytokine concentrations - TNF- $\alpha: 157 \mathrm{pg} / \mathrm{mL}$ and IL-6: 4,700 pg/mL, both of which had a lower detection limit of $30 \mathrm{pg} / \mathrm{mL}$. In investigating solely the role of IL-1 $\beta$ in OA and RA, Richette et al. (247) reported average IL- $1 \beta$ concentrations of $1.0 \mathrm{pg} / \mathrm{mL}$ in the cohort of OA patients $(n=42)$ and average IL-1 $\beta$ concentrations of $24.0 \mathrm{pg} / \mathrm{mL}$ in 
the cohort of RA patients $(\mathrm{n}=11)$ in the synovial fluid, with a lower limit of detection of $0.1 \mathrm{pg} / \mathrm{mL}$. They detailed that detectable levels of IL- $1 \beta$ were found in $71 \%(\mathrm{n}=30 / 42)$ of patients with OA and all patients with RA. In an explorative study comparing levels of cytokines in control and OA synovial fluid, Beekhuizen et al. (248) tested for TNF- $\alpha$, IL$1 \beta$, IL-6, and MCP-1. Their control cohort $(\mathrm{n}=16)$ consisted of synovial fluid collected from the patient within 24 hours post-mortem, whereas the test cohort $(n=18)$ consisted of synovial fluid collected from patients with primary OA. TNF- $\alpha$ levels in both cohorts were below the lower detection limit, which was not detailed. Median IL-1 $\beta$ concentrations were $0.0 \mathrm{pg} / \mathrm{mL}$ in the control cohort and $4.8 \mathrm{pg} / \mathrm{mL}$ in the test cohort with a lower detection limit of $0.7 \mathrm{pg} / \mathrm{mL}$. Median IL-6 concentrations were $4.8 \mathrm{pg} / \mathrm{mL}$ in the control cohort and 135.8 in the test cohort with a lower detection limit of $0.4 \mathrm{pg} / \mathrm{mL}$. Beekhuizen and colleagues (248) reported that IL-6 was significantly elevated in the OA cohort when compared to the control cohort ( $\mathrm{p}=0.001$ ). Lastly, median MCP-1 concentrations were $542.4 \mathrm{pg} / \mathrm{mL}$ in the control cohort and $824.8 \mathrm{pg} / \mathrm{mL}$ in the OA cohort with a lower detection limit of $1.2 \mathrm{pg} / \mathrm{mL}$. Ruth et al. (249) measured MIP-3 $\alpha$ in the synovial fluid from patients with OA $(n=9)$ and patients with RA $(n=25)$ and reported that RA synovial fluid samples exhibited significantly elevated levels of MIP-3 $\alpha(3,001$ $\mathrm{pg} / \mathrm{mL})$ compared to patients with $\mathrm{OA}(100 \mathrm{pg} / \mathrm{mL})$. Although, no value of significance was given.

To determine if IL-1 $\beta$ may be indicative of increased synovial activity, an inflammatory reaction, or osteolysis following TKA, Kovacik et al. (203) compared the IL-1 $\beta$ concentrations in synovial fluid aspirates from OA patients receiving primary TKA with patients receiving revision TKA for aseptic loosening. They reported a significant difference $(\mathrm{p}<0.0001)$ between the average IL- $1 \beta$ concentrations in the OA synovial fluid aspirates $(0.25 \mathrm{pg} / \mathrm{mL})$ compared to the revision synovial fluid aspirates $(6.25 \mathrm{pg} / \mathrm{mL})$. Furthermore, Kovacik et al. (203) found significantly higher IL-1 $\beta$ concentrations $(\mathrm{p}<0.0001)$ in the synovial fluid of the patients receiving revision for failure of a TiAlV component $(9.92 \mathrm{pg} / \mathrm{mL})$ versus revision for failure of a CoCrMo component (2.57 $\mathrm{pg} / \mathrm{mL}$ ). Similarly, Dasa et al. (177) measured the concentrations of TNF- $\alpha$, IL-1 $\beta$, and MCP-1 in patients with $\mathrm{OA}(\mathrm{n}=10)$, patients with $\mathrm{RA}(\mathrm{n}=4)$, and patients with radiographic evidence of aseptic loosening undergoing revision arthroplasty $(\mathrm{n}=6)$. An average TNF- $\alpha$ concentration of $208.0 \mathrm{pg} / \mathrm{mL}$ was reported only for the aseptically failed patient cohort. IL-1 $\beta$ was not detected $(<0.6 \mathrm{pg} / \mathrm{mL})$ in any of the patients undergoing revision TKA. The mean MCP-1 concentration for the revision patients was 21,233 $\mathrm{pg} / \mathrm{mL}$. The mean MCP-1 concentration was $3012 \mathrm{pg} / \mathrm{mL}$ for the OA cohort and 690 $\mathrm{pg} / \mathrm{mL}$ for the RA cohort. Statistical analysis revealed that the mean MCP-1 concentration in revision samples was larger than OA and RA samples ( $\mathrm{p}=0.011$ and $\mathrm{p}=0.014$, respectively), but no difference existed between MCP- 1 in the OA and RA cohorts. Moreover, Dasa et al. (177) noted that patients with OA fell into two distinct subsets based on MCP-1 concentration: greater than 4,000 pg/mL and less than 2,500 $\mathrm{pg} / \mathrm{mL}$. In their investigative study of potential inflammatory biomarkers, Perkins et al. (244) measured TNF- $\alpha$, IL-1 $\beta$, IL-6, MCP-1, MIP-3 $\alpha$, and M-CSF in the synovial fluid of patients with a unexplained, painful TKA $(\mathrm{n}=12)$ and patients receiving revision due to aseptic loosening $(\mathrm{n}=7)$. TNF- $\alpha$ and IL-1 $\beta$ concentrations were below the minimum 
detection limits in both cohorts $(<8.9 \mathrm{pg} / \mathrm{mL}$ and $<16.3 \mathrm{pg} / \mathrm{mL}$, respectively). The average IL-6 concentration for the painful TKA cohort was $572 \mathrm{pg} / \mathrm{mL}$ and for the revision TKA cohort was $476 \mathrm{pg} / \mathrm{mL}$. The average MCP-1 concentration for the painful TKA cohort was $4,481 \mathrm{pg} / \mathrm{mL}$ and for the revision TKA cohort was $4,726 \mathrm{pg} / \mathrm{mL}$. The average MIP-3 $\alpha$ concentration for the painful TKA cohort was $355 \mathrm{pg} / \mathrm{mL}$ and for the revision TKA cohort was $40 \mathrm{pg} / \mathrm{mL}$. Lastly, the average M-CSF concentration for the painful cohort was $1,559 \mathrm{pg} / \mathrm{mL}$ and for the revision TKA cohort was $1,663 \mathrm{pg} / \mathrm{mL}$. No statistical differences were detected (244).

To the author's knowledge, the present study is the first to document TNF- $\alpha$, IL$1 \beta$, IL-6, MCP-1, MIP-3 $\alpha$, and M-CSF in the synovial fluid aspirated post-mortem from specimens with well-functioning primary TKAs. Ten specimens $(31 \%, n=10 / 32)$ had TNF- $\alpha$ concentrations below the minimum detection limit $(8.9 \mathrm{pg} / \mathrm{mL})$. The remaining $69 \%(\mathrm{n}=22 / 32)$ specimens had a median TNF- $\alpha$ concentration of $12.9 \mathrm{pg} / \mathrm{mL}$ which is much lower than the concentration reported for the RA cohort $(157 \mathrm{pg} / \mathrm{mL})$ in the study by Steiner et al. (246) as well as the concentration reported for the revision cohort (208 $\mathrm{pg} / \mathrm{mL}$ ) in the study by Dasa et al. (177). Whereas the previous studies reported IL-1 $\beta$ concentrations ranging from $0.00 \mathrm{pg} / \mathrm{mL}$ (248) to $24 \mathrm{pg} / \mathrm{mL}$ (247) in OA, RA, and necropsy control patients, the present study found a median IL-1 $\beta$ concentration of 73.2 $\mathrm{pg} / \mathrm{mL}$ with $85 \%(\mathrm{n}=28 / 32)$ of the specimens having concentrations above the minimum detection limit (16.3 pg/mL). TNF- $\alpha$ and IL-1 $\beta$ have both been well established as mediators in osteolysis through modulation of the RANK/RANKL/OPG axis. These findings demonstrate near-basal levels present in the well-functioning necropsy specimens. This is expected, however, because the patients included in the present study were well-functioning as dictated by no evidence of radiolucencies on the fluoroscopes taken at retrieval. An additional factor that may modulate the activity of the cytokines is the fact that their presence is likely transient in the inflammatory process. Wear debris is generated, macrophages are stimulated, cytokines are released, macrophages and other immune cells travel to the site of aggravation, and eventually, anti-inflammatory, homeostatic mechanisms begin, restoring the equilibrium. Some of the specimens in this study may have been collected when they were in the aggravation time-point whereas others may have been collected when they were in the anti-inflammatory, homeostatic time-point.

IL-6, in particular, has been implicated in modulating the response to periprosthetic infections in joint arthroplasties (250-252). However, IL-6 is also regarded as a pleiotropic cytokine whose duty is to "ramp" up the inflammatory response whether it be in the pro- or anti-inflammatory direction (253). Six specimens had IL-6 concentrations above the maximum detection limit $(1180 \mathrm{pg} / \mathrm{mL})$. The median IL-6 concentration reported fell between the IL-6 concentrations reported in the post-mortem control cohort and the OA cohort of the study by Beekhuizen et al. (248). The concentrations fell into two subsets: those greater than $400 \mathrm{pg} / \mathrm{mL}$ and those less than $400 \mathrm{pg} / \mathrm{mL}$. This follows what was reported by Mabey et al. and Perkins et al. in which the OA, painful, and revision cohorts averaged around 400 pg/mL (244). IL-6 concentrations exceeding 400 $\mathrm{pg} / \mathrm{mL}$ may be indicative of the inflammatory process "ramping" up. With respect to MCP-1, all but one specimen had concentrations above the minimum detection limit (98 
$\mathrm{pg} / \mathrm{mL})$. Furthermore, 8 specimens had MCP-1 concentrations over the maximum detection limit $(7940 \mathrm{pg} / \mathrm{ml})$. Similar to what was found in the study by Dasa et al. (177), the MCP-1 concentrations for these specimens seem to fall into two separate subsets: those greater than $4000 \mathrm{pg} / \mathrm{mL}$ and those less than $4000 \mathrm{pg} / \mathrm{mL}$. More specifically, all of the specimens with MCP-1 levels greater than $4000 \mathrm{pg} / \mathrm{mL}$ surpassed the detection limit of the assay platform $(>7940 \mathrm{pg} / \mathrm{mL})$. This may indicate a certain stimulus threshold exists at which macrophages will increase the production of MCP-1.

MIP-3 $\alpha$ is a chemokine that is strongly chemotactic for lymphocytes and has been implicated in modulating the adaptive immune system (254) Additionally, MIP-3 $\alpha$ is chemotactic for leukocytes to joints afflicted by RA (249). As such, it follows logically that the MIP-3 $\alpha$ levels reported in the present study would align with those reported in literature for OA, painful, or revision patients, while the MIP-3 $\alpha$ concentrations in patients with RA would be elevated $(244,249)$. Recent research indicates that GM-CSF polarizes $\mathrm{M}_{0}$ macrophages into $\mathrm{M}_{1}$ (pro-inflammatory) phenotype whereas $\mathrm{M}$-CSF polarizes $\mathrm{M}_{0}$ macrophages into $\mathrm{M}_{2}$ (healing) phenotype (255). None of the specimens of each sample group had GM-CSF concentrations above the minimum detection limit. These results are in accordance with previous studies that demonstrated negligible levels of GM-CSF in loosened THA and fixed THA $(256,257)$. On the other hand, all of the specimens had M-CSF concentrations that were within range. The present study found noticeably elevated levels of M-CSF in the synovial fluid of cadaveric primary TKAs compared to the painful and revision synovial fluid reported in Perkins et al. (244). Each cadaveric sample had M-CSF concentrations that were an order of magnitude greater than those observed in the living patients. While previous studies found elevated levels of MCSF in aseptically loosened THAs compared to well-fixed THAs, this present study does not support this notion with respect to TKAs $(258,259)$. Future research may investigate the roles of GM-CSF and M-CSF concentrations as indicators as to whether the body is entering a cyclic inflammatory cascade, which may precede failure, or if the body is simply going through the normal inflammation-healing sequence.

Certain limitations of cytokine and inflammatory marker measurements in clinical research must be acknowledged. Many cytokines have a short half-life in vivo and are also subject to rapid degradation in vitro following sample collection if appropriate storage and handling procedures are not followed (260-263). While literature has not elucidated the effect of processing and storage on the degradation of cytokines in synovial fluid, several reports on serum and plasma have indicated that most cytokines are prone to degradation if samples are not processed correctly and stored at $-70^{\circ} \mathrm{C}(264-$ 266). Furthermore, the amount of freeze-thaw cycles has been demonstrated to effect cytokine measurements. Thavasu et al. (266) found that most cytokines are stable for up to three freeze-thaw cycles. However, Flower et al. (267) found that TNF- $\alpha$ concentration increased with each successive free-thaw cycle, becoming significant after three cycles. Time of day of sample collection has also been demonstrated to impact reported levels of circulating cytokines $(262,268-270)$. While every effort was taken to mitigate the aforementioned limitations, measured cytokine levels in the synovial fluid of this study cohort may not entirely reflect the presence or activity of those cytokines in vivo. 


\section{Joint Laxity}

Suboptimal soft tissue and gap balancing, which dictate the laxity of the joint, may be implicated in the etiology of some of the most common indications for revisionloosening, excessive wear, instability, and malalignment (116-122). The IE, VV, and AP laxity were measured using a platform that has been previously studied and utilized in previously published biomechanical studies of TKA kinematics $(114,184-193)$. Of the studies cited, only four provided data points that were used for comparison to the present study (184, 187-189). Contrary to the present study, Jojima et al. (188) evaluated the IE laxity at $\pm 5 \mathrm{Nm}$, VV laxity at $\pm 15 \mathrm{Nm}$, and AP laxity at $\pm 70 \mathrm{~N}$. Similarly Kanamiya et al. (189) applied a $\pm 10 \mathrm{Nm}$ torque to evaluate rotational laxity, as shown in Figures 4-1 through 4-3. While there were great variations in IE and VV laxity, across the flexion angles between each of the studies, the current study follows the trend of increasing IE and VV laxity throughout the flexion angles. Furthermore, the current study PS implants had maximum AP laxity at 30, which is in accordance with the PS implants evaluated by Saeki et al. (187). The trend of CR implant laxity reported in the current study closely mimics that which was reported by Jojima et al. (188) in their evaluation of CR implant designs although their AP laxity was measured at $\pm 70 \mathrm{~N}$. Although similar trends were observed, there is a significant difference between the cited studies and the present study which likely affects the kinematics of the specimens. The specimens used by Saeki et al. (187), Kanamiya et al. (189), and Jojima et al. (188) were five fresh-frozen knee specimens who received total knee arthroplasty post-mortem. As such, the incisions and trauma sustained during TKA were not healed. Additionally, the cited studies all used the Profix total knee system (Smith \& Nephew Inc, Memphis, TN). The current study included several different implant designs from various manufacturers. Because knee kinematics is affected by the implant design $(72,271)$, this variation must be considered in the comparisons. A previous graduate student in Dr. Mihalko's laboratory, Erik Woodard (184), evaluated IE, VV, and AP laxity using the same simulator and at the same IE and VV torque and AP force poles as the present study. However, his cohort included both CR and PS implant designs. As such, the comparison between his study and the present study is presented in Figures 4-4 through 4-6, in which the PS and CR designs were included together. Again, the IE, VV, and AP laxity reported for both the current study and the study by Woodard (184) follow the similar trends. Similar to the present study, Woodard (184) included a variety of implant designs from several manufacturers.

\section{Correlation of Study Parameters}

\section{Hypothesis (a)—_Laxity and Tissue Metal Concentrations}

Excessive ligamentous laxity has been reported to increase AP translation and IE rotation which leads to force concentrations that are outside of the intended design parameters of the TKA components. More specifically, researchers found that these force concentrations exceeded the material properties of the tibial insert facilitating increased 


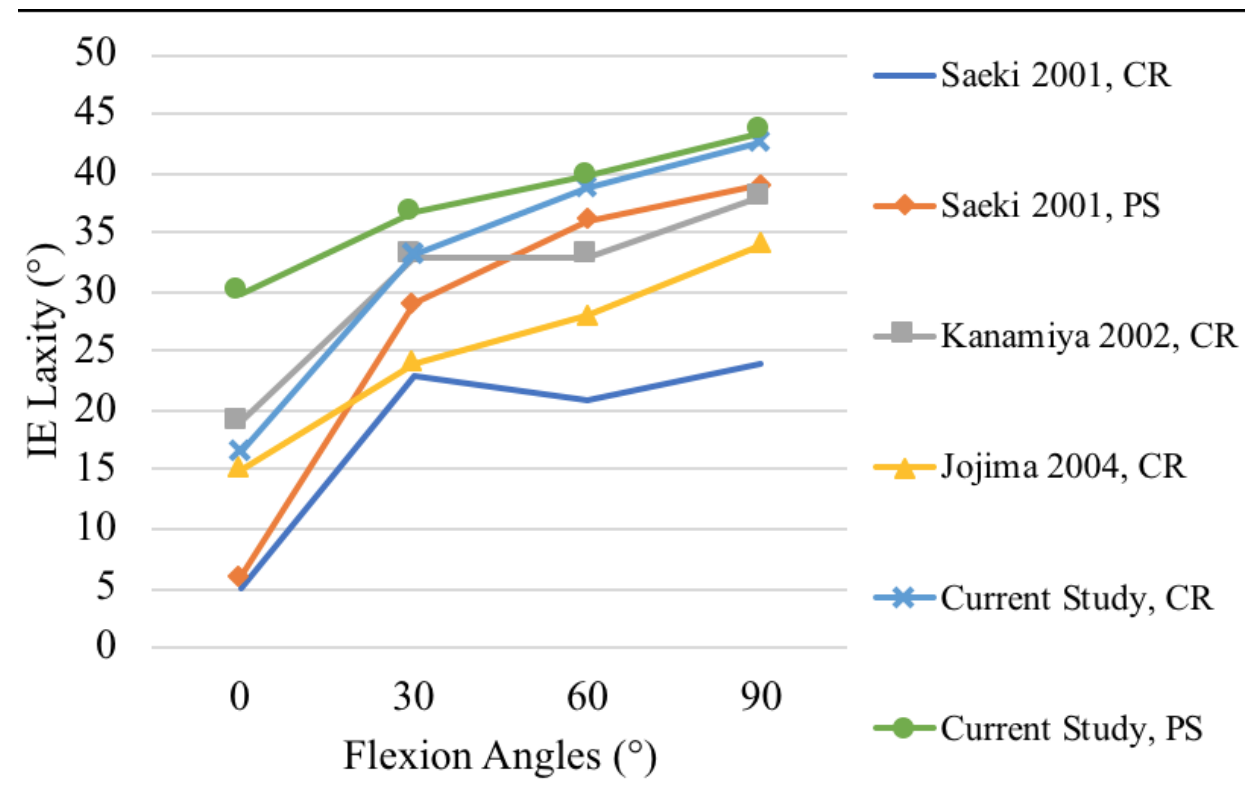

Figure 4-1. IE rotational laxity comparison between the present study and Saeki et al., Kanamiya et al., and Jojima et al.

Sources:

Jojima H, Whiteside LA, Ogata K. Effect of tibial slope or posterior cruciate ligament release on knee kinematics. Clinical orthopaedics and related research. 2004;426:194-8.

Kanamiya T, Whiteside LA, Nakamura T, Mihalko WM, Steiger J, Naito M. Effect of Selective Lateral Ligament Release on Stability in Knee Arthroplasty. Clin Orthop Relat Res. 2002;404:24-31.

Saeki K, Mihalko WM, Patel V, Conway J, Naito M, Thrum H, et al. Stability after medial collateral ligament release in total knee arthroplasty. Clinical orthopaedics and related research. 2001;392:184-9. 


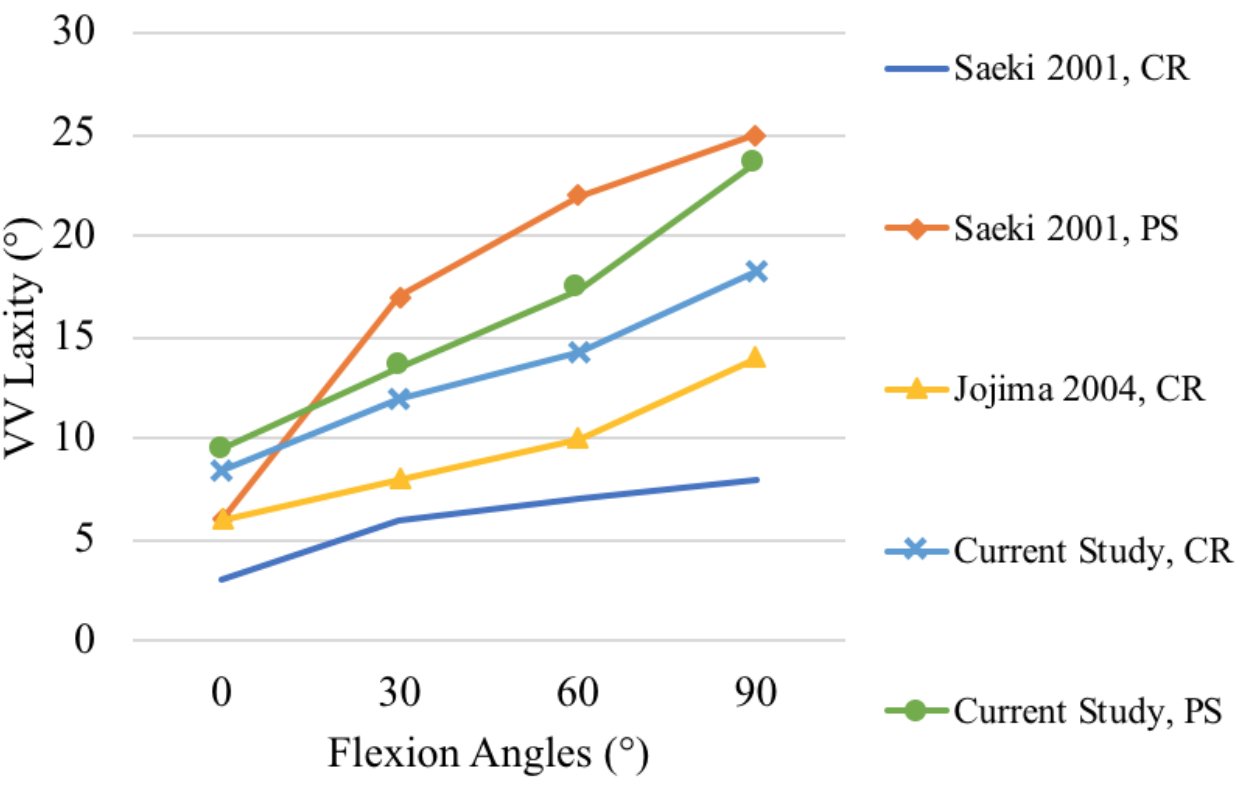

Figure 4-2. VV laxity comparison between the present study and Saeki et al. and Jojima et al.

Sources:

Jojima H, Whiteside LA, Ogata K. Effect of tibial slope or posterior cruciate ligament release on knee kinematics. Clinical orthopaedics and related research. 2004;426:194-8.

Saeki K, Mihalko WM, Patel V, Conway J, Naito M, Thrum H, et al. Stability after medial collateral ligament release in total knee arthroplasty. Clinical orthopaedics and related research. 2001;392:184-9. 


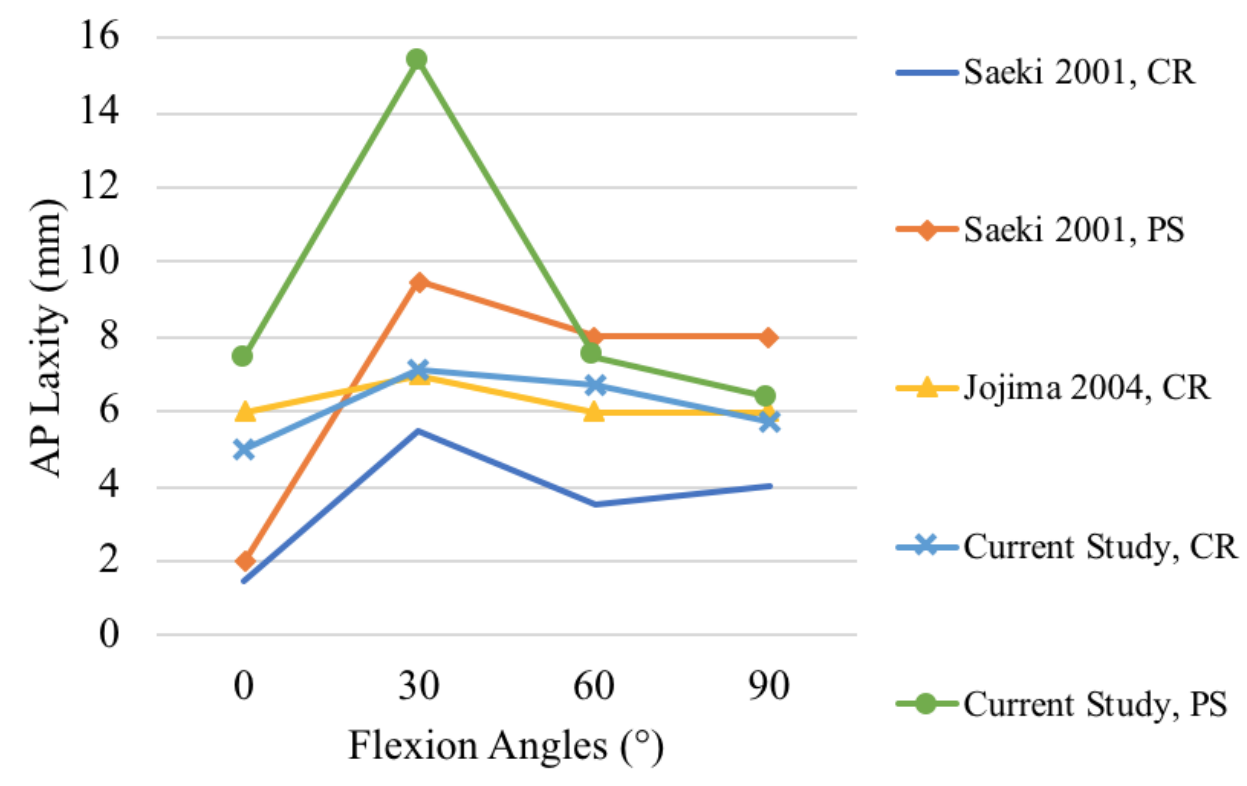

Figure 4-3. Comparison of measured AP laxity between current study, Saeki et al. and Jojima et al.

Sources:

Jojima H, Whiteside LA, Ogata K. Effect of tibial slope or posterior cruciate ligament release on knee kinematics. Clinical orthopaedics and related research. 2004;426:194-8.

Saeki K, Mihalko WM, Patel V, Conway J, Naito M, Thrum H, et al. Stability after medial collateral ligament release in total knee arthroplasty. Clinical orthopaedics and related research. 2001;392:184-9. 


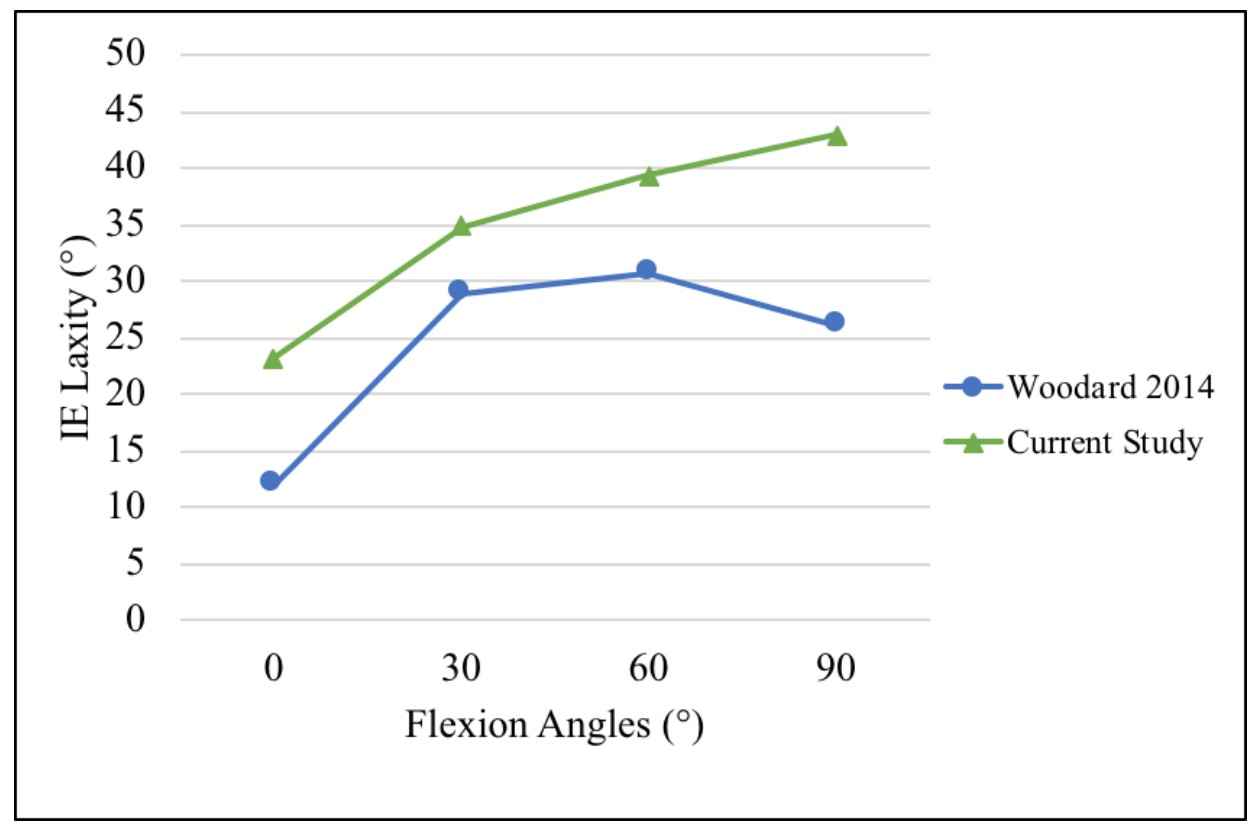

Figure 4-4. Comparison of IE laxity between current study and Woodard

Source: Woodard EL. Total knee arthroplasty wear is caused by malrotation and excessive laxity [Theses and Dissertations (ETD)]. Memphis, TN: University of Tennessee Health Science Center; 2014. 


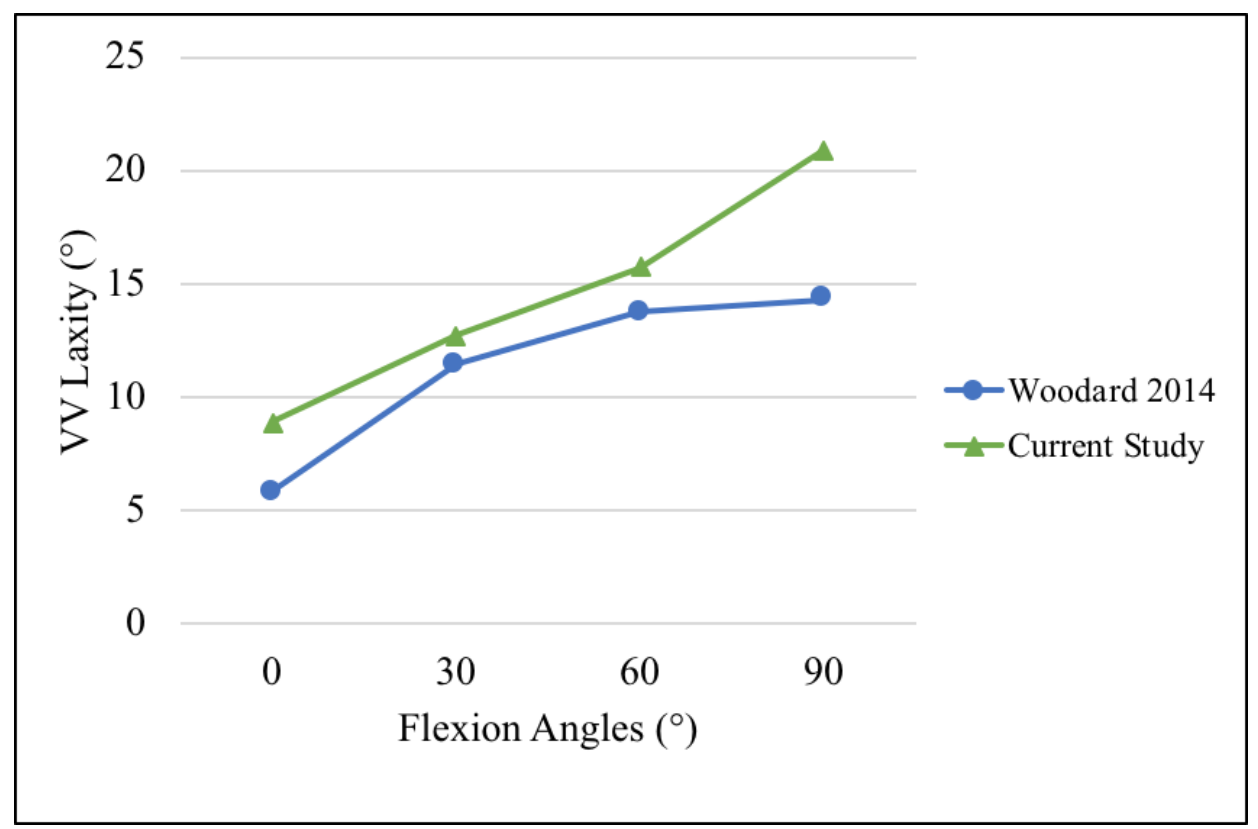

Figure 4-5. Comparison of VV laxity between current study and Woodard

Source: Woodard EL. Total knee arthroplasty wear is caused by malrotation and excessive laxity [Theses and Dissertations (ETD)]. Memphis, TN: University of Tennessee Health Science Center; 2014. 


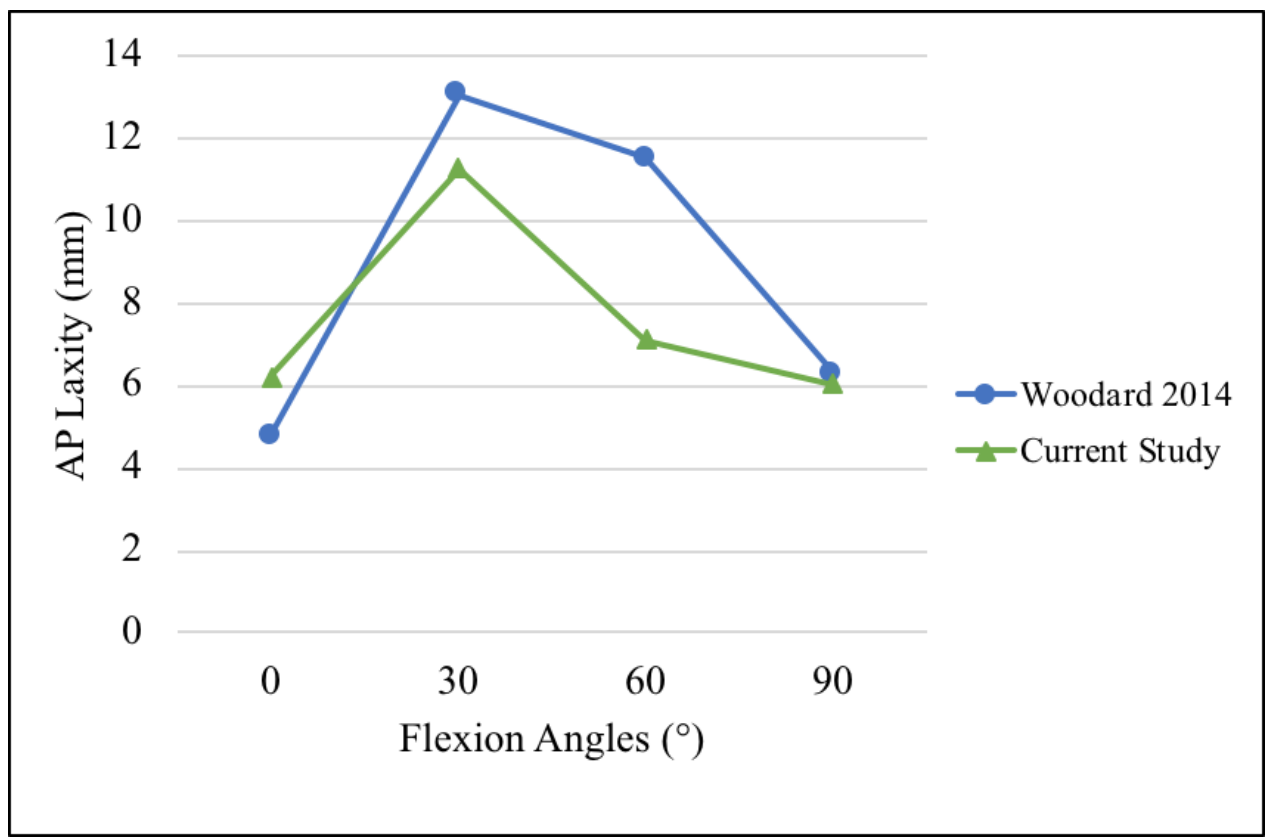

Figure 4-6. Comparison of AP laxity between current study and Woodard

Source: Woodard EL. Total knee arthroplasty wear is caused by malrotation and excessive laxity [Theses and Dissertations (ETD)]. Memphis, TN: University of Tennessee Health Science Center; 2014. 
UHMWPE wear (78, 123-126). However, there is a paucity of literature evaluating the relationship between joint laxity and metallic wear debris production following TKA. It was hypothesized that decreased joint laxity would increase metal concentrations in the periprosthetic tissue.

In the present study, decreased IE rotation at full extension was strongly correlated with increased Co concentrations in the periprosthetic tissues. When taking a closer look at the correlation, constrained external rotation specifically induced elevated concentrations of Co. While not statistically significant, a similar trend was observed between IE laxity at full extension and Cr. The PCL and the posterolateral corner work synergistically to control external rotation whereas the ACL is the primary stabilizer to internal rotation. In a normal flexion/extension cycle, the tibia externally rotates $5^{\circ}$ in the last $15^{\circ}$ of extension in the "screw home" mechanism. With the application of $1.5 \mathrm{Nm}$ torque, this study observed that the primary TKA specimens were able to achieve a median external rotation of $10.18^{\circ}$. However, when comparing the external rotation to the $\mathrm{Co}$ and $\mathrm{Cr}$ concentrations observed, decreased external rotation in full extension was associated with elevated $\mathrm{Co}$ and $\mathrm{Cr}$ concentrations. The decreased ability of the knee to externally rotate may have increased the contact pressures on the bearing surface facilitating abrasive wear of the CoCrMo bearing surface. Elevated concentrations of Co indicate that wear may be dominant within the cobalt rich matrix of the CoCrMo alloy. This agrees with observations reported by Kretzer et al. (232) in their in vitro analysis of metal release kinetics in TKA using a wear simulator.

Statistical significance was not detected in correlations between VV laxity and $\mathrm{Co}, \mathrm{Cr}$, or Ti debris. However, at full extension and $30^{\circ}$ of flexion, the relationship between VV laxity and Ti debris in the periprosthetic tissues displayed an inverse trend. All but two implants had femoral components fabricated from CoCrMo alloy. The implant with TiAlV femoral component was excluded from the statistical analysis due to extremely elevated $\mathrm{Ti}$ concentrations. As such, the only material that could contribute to the generation of Ti debris is the baseplate. In this comparison, 6 of the 9 PS implant systems had CoCrMo femoral components with TiAlV tibial baseplates, while the remaining 3 had CoCrMo baseplates. Furthermore, 3 of 5 CR implant systems had CoCrMo tibial baseplates, while the remaining 2 were an all-UHMWPE monoblock component and TiAlV baseplate. When analyzing Figures B-11A and B-11B, the posterior-stabilized implants seem to be the major contributing factor to this trend. The primary stabilizers to VV deflection is the MCL and LCL. Additionally, the secondary function of the cruciate ligaments is to resist VV motion at full extension. When the ACL and PCL are sacrificed, as in patients receiving a PS design, the post also must resist this motion. In response to VV stresses, articulation of post with the medial and lateral sides of the femoral component between the bearing surfaces may increase loads in the transverse plane. This may inadvertently cause micromotion between the inferior surface of the tibial insert and the superior surface of the tibial baseplate, facilitating the release of Ti. 
Because the study population included both PS and CR implant designs, anterior translation and posterior translation were compared separately with tissue metal across the flexion angles. Across each flexion angle, the relationship between anterior translation and Co debris strongly trended towards an inverse correlation, as shown in Figures B-13 and B-14. Statistical analysis highlighted significance between decreased anterior laxity at $90^{\circ}$ and increased Co debris. Similarly, statistical significance was revealed between decreased anterior laxity at $60^{\circ}$ and $90^{\circ}$ of flexion and increased $\mathrm{Cr}$ concentrations in the periprosthetic tissues. Most implant designs require the sacrifice of the ACL, creating an inherent deficiency in resisting anterior translation. To compensate for ACL deficiency, many designs have a more dished surface on condylar surface of the tibial insert. Decreased anterior translation, particularly at $60^{\circ}$ and $90^{\circ}$ of flexion, may increase wear of the femoral component causing the release of $\mathrm{Cr}$-rich carbides which then abrade the Co-rich matrix of the CoCrMo alloy, elevating the release of Co. Interestingly, a strong trend of inverse correlations between posterior laxity across the measured flexion angles and $\mathrm{Cr}$ concentrations was observed. Statistical analysis highlighted a significant relationship between decreased posterior laxity at $90^{\circ}$ of flexion and increased $\mathrm{Cr}$ concentrations in the periprosthetic tissues. When looking at the graphical representation of the correlation at $90^{\circ}$ of flexion in Figure B-20B, both the CR and PS designs seem to follow the negative trend. In a cruciate-retaining TKA, the primary stabilizer to posterior displacement, especially at $90^{\circ}$ of flexion, is the PCL. However, in a cruciate-sacrificing design, the post engages the cam at $90^{\circ}$ of flexion, preventing posterior translation. Regardless if the implant is a cruciate-retaining or cruciate-sacrificing design, these results suggest that decreased posterior translation may induce increased release of $\mathrm{Cr}$ debris.

\section{Hypothesis (b)_Laxity and Inflammatory Cytokines}

As previously stated, suboptimal soft tissue laxity has been demonstrated to influence the degree of polyethylene wear, such that excessive AP and IE laxity induced elevated rates of polyethylene wear due to the contact forces that exceed the design parameters of the insert $(78,123-126)$. Historically, wear-induced osteolysis and aseptic loosening was attributed to excessive polyethylene wear. In 2002, Sharkey et al. (272) reported polyethylene wear was the most common cause of failure in TKA, accounting for almost $25 \%$ of all revisions. Recently, however, the manufacturing of polyethylene has improved, with particular attention paid to the sterilization and packaging techniques. It was found that during shelf storage, UHMWPE inserts that were gamma sterilized in air-permeable packaging could undergo oxidative degradation, resulting in an increase in density and crystallinity; more importantly, the oxidative degradation led to a loss of mechanical properties associated with progressive embrittlement $(213,215,273,274)$. As such, air-permeable packaging was replaced by barrier packaging with a low oxygen environment starting during the mid 1990s (204). In 2014, Sharkey et al. (275) repeated a systematic retrospective review of all revisions performed at their institution to see if the reasons for revision have changed in the past 10 years. They found that polyethylene wear was no longer a major cause for failure while aseptic loosening accounted for $40 \%$ of all revisions at their institution (275). The reduction in polyethylene wear-related 
failures is likely due to changes in sterilization and storage techniques and improved locking mechanisms. Because aseptic loosening still persists despite improvements in articulating materials and increased laxity has been reported to elevate polyethylene wear, it was hypothesized that a direct correlation would be observed between increased laxity and elevated inflammatory cytokines.

An inverse correlation was observed between IE rotational laxity at $60^{\circ}$ of flexion and IL-1 $\beta$ concentrations in Figure C-4A. IL- $1 \beta$ is a cytokine that has been well established as a mediator of osteolysis (170). Furthermore, macrophages have been shown to release IL-1 $\beta$ in response to UHMWPE debris $(166,276)$. If increased IE laxity induces UHMWPE wear, then it would follow that increased laxity would also induce elevation of inflammatory cytokine concentrations. As such, the inverse correlation was unexpected. Further statistical analysis determined that this trend was strongly influenced by the CR implants and the trend did not persist with the removal of implant RLU0722161B-L, an all-polyethylene monoblock design, that had extremely restricted IE rotation at $60^{\circ}$ of flexion and the highest IL- $1 \beta$ concentration of $962 \mathrm{pg} / \mathrm{mL}$. This data point likely was pulling the trend for inverse correlation.

To date, no studies have demonstrated the relationship between degree of laxity in the coronal plane and wear of the TKA components. This study found that, at $60^{\circ}$ of flexion, VV laxity was inversely correlated with TNF- $\alpha$ concentrations as shown in Figure C-14A. However, it must be noted that levels of TNF- $\alpha$ were barely above the minimum detection limit; as such, this correlation was likely clinically insignificant. Additionally, VV laxity was inversely correlated with IL- $1 \beta$ concentrations at $60^{\circ}$ of flexion as shown in Figure C-16A. Again, the specimen RLU0722161B-L had extremely restricted VV rotation at $60^{\circ}$ of flexion and the highest IL- $1 \beta$ concentration of 962 $\mathrm{pg} / \mathrm{mL}$. This data point likely was pulling the trend for inverse correlation. At full extension, VV laxity was inversely correlated with IL-6 concentrations. This correlation seems to be due to specimens RLU0317172B-R and RLU1121161A-L which had IL-6 concentrations above the maximum detection limit $(>1180 \mathrm{pg} / \mathrm{mL})$ and very constrained VV deflection at full extension. These two data points seem to be pulling the trend for this inverse correlation. In conclusion, none of the correlations reported between VV laxity and inflammatory cytokine concentrations likely have any clinical implications.

Anterior laxity was directly correlated with IL-6 and MCP-1 concentrations at $90^{\circ}$ of flexion, shown in Figures C-34B and C-38B, respectively. Additionally, posterior laxity was directly correlated with IL-6 at $30^{\circ}$ and $90^{\circ}$ of flexion as shown in Figures C35B and C-36B, respectively, although statistical significance was not detected. Both IL6 and MCP-1 have been implicated in the response to UHMWPE debris $(166,177)$. Kaufman et al. (166) posited that IL-6 and MCP-1 may function cooperatively at the periimplant tissue; MCP-1 actively recruits macrophages to the site of inflammation while IL-6 potently enhances the recruitment of osteoclast precursors and promotes osteoclastogenesis $(172,253,277)$. These findings suggest that increased AP translation may increase the production of UHMWPE debris which incites an inflammatory response and the subsequent release of IL-6 and MCP-1. 


\section{Hypothesis (c)—Tissue Metal Concentrations and Inflammatory Cytokines}

The in vivo biological response to wear debris is complex, involving a range of cell types, including fibroblasts, osteoclast precursor cells, and monocytes/macrophages, that may release a variety of inflammatory mediators including, cytokines, chemokines, prostanoids, reactive oxygen species, and MMPs, into the synovial fluid and periprosthetic tissues. To the author's knowledge, this is the first study to measure the concentrations of a various inflammatory mediators in the synovial fluid of patients with well-functioning primary TKA at autopsy. Furthermore, this is the first study to examine the relationships of in vivo tissue metal concentrations and the levels of these inflammatory mediators. Several in vitro studies have demonstrated that macrophages will increase their release or expression of the cytokines included in this study when challenged with Ti or CoCr wear particles (150, 166, 176, 276, 278-280). Also, each of these cytokines or chemokines have been demonstrated in retrieved at revision in tissues or synovial fluid from patients with aseptically loosened primary total hip and total knee implants $(177,181,203,244,257,258,281)$. As such, it was hypothesized that a direct correlation would be observed between the inflammatory cytokines and tissue metal concentrations.

No significant correlations were found between Co concentrations and inflammatory cytokines. The periprosthetic tissue $\mathrm{Cr}$ concentrations displayed a trend of inverse correlation with IL-1 $\beta$, IL-6, MCP-1, and MIP-3 $\alpha$ concentrations in the synovial fluid, although significance was only detected for IL-6 and MIP-3 $\alpha$. Similarly, Ti was trending towards an inverse correlation with IL-1 $\beta$ and MIP-3 $\alpha$, although statistical significance was not detected. These results may be interpreted in many ways. The inverse trend suggests that the cytokines measured may not be particularly sensitive to metallic debris. However, when investigating the human macrophage response to UHMWPE, TiAlV, CoCrMo, and alumina particles in vitro, Kaufman et al. (166) reported that TiAlV debris consistently elicited the release of the cytokines of interest and was the second most stimulatory species behind the positive control, lipopolysaccharide (LPS). After TiAlV challenge, TNF- $\alpha$ (895-fold), IL-1 $\beta$ (46-fold), IL-6 (20-fold), IL-8 (5-fold), and MCP-1 (19-fold) release was increased at a statistically significant level when compared to the nonstimulated macrophages that served as a negative control (166). They found that the CoCr particles were less inflammatory than the Ti particles and only marginally more inflammatory than the UHMWPE particles. The CoCrMo particulates only elicited significant increases in TNF- $\alpha$, IL-6, and IL-8 release when compared to the nonstimulated cells; however, the fold-increase was 17-fold, 2.3-fold, and 2.3-fold, which is vastly lower than the fold-increase reported for TiAlV challenge (166). This study demonstrates that each of these cytokines are released by macrophages when challenged with Ti particles and $\mathrm{CoCr}$ particles although to varying degrees. Thus, it is unlikely that the $\mathrm{Ti}, \mathrm{Co}$, and $\mathrm{Cr}$ particles present in the tissues of the present study's cohort did not stimulate an inflammatory response. Instead, some of the patients in the present study may not have been in an actively "stimulated" inflammatory state. The cycle of inflammation is transient and the levels of inflammatory cytokines will rise and fall as the cycle transitions from a pro-inflammatory to anti-inflammatory state. The 
cytokine release observed by Kaufman et al. (166) was measured within a 24 hour time period when the macrophages were likely at their "stimulated" inflammatory peak state. Wang et al. (282) investigated how $\mathrm{Ti}, \mathrm{Cr}$, and Co ions modulate the release of boneassociated cytokines by human monocytes/macrophages in vivo. Similar to Kaufman et al. (166), Wang and colleagues (282) demonstrated that $\mathrm{Ti}, \mathrm{Cr}$, and Co modulate cytokine release, specifically TNF- $\alpha$, IL-1 $\beta$ and IL-6, in LPS-stimulated cells. However, they reported that cytokine production was not affected by the metals in the absence of LPS. This finding highlights the fact that often the inflammatory process is like a light switch: it is either "on" or "off". The correlations observed in the present study may have been induced by prior stimulation by other types of wear debris in the periarticular environment.

\section{Limitations}

While some meaningful relationships were observed in this study, there were several limitations that must be noted. Firstly, this study had a relatively small sample size. Furthermore, not every specimen underwent each of the analyses or tests. As such, some important correlations may have not been recognized in statistical analysis or the power of the observations may not be clinically significant. Secondly, the cohort included a wide range of implants including cruciate-retaining, posterior-stabilized, fixed bearing, mobile bearing, monoblock, and modular designs. Each of these design variations have been shown to affect the kinematics of the knee joint. As such, it is difficult to determine how the design may have affected the measured laxity of the joint or the degree of wear debris generated. Furthermore, one of the specimens included was produced in the late 1980 s or early 1990s by a manufacturer that no longer exists. Additionally, the implants included were fabricated with different materials. However, every effort was made to take into account these variations and discuss how the different designs may have impacted the results of the study. Thirdly, because the Hood et al. (195) method for wear analysis is essentially qualitative, it was impossible to take into account how the degree of polyethylene debris produced from the tibial insert may have altered the cytokine measurements or even the laxity. Again, every effort was made to note instances of excessive wear when meaningful relationships were observed. Fourthly, many variables were uncontrollable in the collection of the synovial fluid and measurement of inflammatory cytokines. These limitations were previously discussed and every effort was taken to mitigate them. Lastly, the state of the measured metal debris (e.g. large metal particulate or metal oxide) could not be elucidated with the current methods represented in this study. The ICP-MS analysis technique ionizes all metal present and reports a final concentration; it does not differentiate between larger particles or ions.

\section{Future Research}

Dr. Mihalko's laboratory has obtained institution review board approval and has begun to collect revision devices. Currently, the sample size is small, but is anticipated to expand in the upcoming months. Once a sufficient sample size is achieved, the graduate 
student researchers at University of Tennessee Health Science Center and Drexel University will begin assessing the wear and damage of these devices. Furthermore, a cytokine analysis will be performed to measure the levels of inflammatory cytokines and ICP-MS analysis will be conducted to measure the concentrations of metal debris in the collected tissues. Future comparisons between the two cohorts will hopefully elucidate what factors are instrumental in mediating negative outcomes so that one day a treatment modality, alternate bearing surface, or improved surgical technique may be developed to improve patient outcomes following total knee arthroplasty. 


\section{LIST OF REFERENCES}

1. Kremers HM, Larson DR, Crowson CS, Kremers WK, Washington RE, Steiner CA, et al. Prevalence of total hip and knee replacement in the United States. J Bone Joint Surg Am. 2015;97(17):1386.

2. Kurtz S, Ong K, Lau E, Mowat F, Halpern M. Projections of primary and revision hip and knee arthroplasty in the United States from 2005 to 2030. J Bone Joint Surg. 2007;89(4):780-5.

3. Gibon E, Goodman MJ, Goodman SB. Patient Satisfaction After Total Knee Arthroplasty: A Realistic or Imaginary Goal? Orthop Clin North Am. 2017;48(4):421-31.

4. Wylde V, Hewlett S, Learmonth ID, Dieppe P. Persistent pain after joint replacement: prevalence, sensory qualities, and postoperative determinants. PAIN. 2011;152(3):566-72.

5. Beswick AD, Wylde V, Gooberman-Hill R, Blom A, Dieppe P. What proportion of patients report long-term pain after total hip or knee replacement for osteoarthritis? A systematic review of prospective studies in unselected patients. BMJ open. 2012;2(1):e000435.

6. Bourne RB, Chesworth B, Davis A, Mahomed N, Charron K. Comparing patient outcomes after THA and TKA: is there a difference? Clin Orthop Relat Res. 2010;468(2):542-6.

7. Bhandari M, Smith J, Miller LE, Block JE. Clinical and economic burden of revision knee arthroplasty. Clin Med Insights Arthritis Musculoskelet Disord. 2012;5:89.

8. Mulhall KJ, Ghomrawi HM, Scully S, Callaghan JJ, Saleh KJ. Current etiologies and modes of failure in total knee arthroplasty revision. Clin Orthop Relat Res. 2006;446:45-50.

9. Mihalko WM. Arthroplasty of the Knee. In: Azar FM, Beaty JH, Canale ST, editors. Campbell's Operative Orthopaedics. 11 ed. Philadelphia, PA: Elsevier; 2017. p. 395-468.

10. Revell PA. The combined role of wear particles, macrophages and lymphocytes in the loosening of total joint prostheses. J R Soc Interface. 2008;5(28):1263-78.

11. Freeman MA, Pinskerova V. The movement of the normal tibio-femoral joint. J Biomech. 2005;38(2):197-208.

12. Dennis DA, Komistek RD, Hoff WA, Gabriel SM. In vivo knee kinematics derived using an inverse perspective technique. Clin Orthop Relat Res. 1996;331:107-17.

13. Komistek RD, Dennis DA, Mahfouz M. In vivo fluoroscopic analysis of the normal human knee. Clin Orthop Relat Res. 2003;410:69-81.

14. Grood E, Suntay W. A joint coordinate system for the clinical description of three-dimensional motions: application to the knee. J Biomech Eng. 1983;105:136.

15. Foran JR. Total Knee Replacement: AAOS; 2015 [

16. Bingham C. Rheumatoid Arthritis Information 2013 [ 
17. Buckwalter JA, Saltzman C, Brown T. The impact of osteoarthritis: implications for research. Clin Orthop Relat Res. 2004;427:S6-S15.

18. Schiphof D, Boers M, Bierma-Zeinstra SM. Differences in descriptions of Kellgren and Lawrence grades of knee osteoarthritis. Ann Rheum Dis. 2008;67(7):1034-6.

19. Kellgren J, Lawrence J. Radiological assessment of osteo-arthrosis. Ann Rheum Dis. 1957;16(4):494.

20. Richmond J, Hunter D, Irrgang J, Jones MH, Snyder-Mackler L, Van Durme D, et al. American Academy of Orthopaedic Surgeons clinical practice guideline on the treatment of osteoarthritis (OA) of the knee. J Bone Joint Surg. 2010;92(4):990-3.

21. Polkowski GG, Ruh EL, Barrack TN, Nunley RM, Barrack RL. Is pain and dissatisfaction after TKA related to early-grade preoperative osteoarthritis? Clin Orthop Relat Res. 2013;471(1):162-8.

22. Schnurr C, Jarrous M, Güdden I, Eysel P, König DP. Pre-operative arthritis severity as a predictor for total knee arthroplasty patients' satisfaction. Int Orthop. 2013;37(7):1257-61.

23. Valdes AM, Doherty SA, Zhang W, Muir KR, Maciewicz RA, Doherty M, editors. Inverse relationship between preoperative radiographic severity and postoperative pain in patients with osteoarthritis who have undergone total joint arthroplasty. Seminars in arthritis and rheumatism; 2012: Elsevier.

24. Cushnaghan J, Bennett J, Reading I, Croft P, Byng P, Cox K, et al. Long-term outcome following total knee arthroplasty: a controlled longitudinal study. Ann Rheum Dis. 2009;68(5):642-7.

25. Dowsey M, Nikpour M, Dieppe P, Choong P. Associations between pre-operative radiographic changes and outcomes after total knee joint replacement for osteoarthritis. Osteoarthritis Cartilage. 2012;20(10):1095-102.

26. Mihalko WM, Whiteside LA. Bone resection and ligament treatment for flexion contracture in knee arthroplasty. Clin Orthop Relat Res. 2003;406(1):141-7.

27. Ritter MA, Lutgring JD, Davis KE, Berend ME, Pierson JL, Meneghini RM. The role of flexion contracture on outcomes in primary total knee arthroplasty. $\mathrm{J}$ Arthroplasty. 2007;22(8):1092-6.

28. Tew M, Forster I. Effect of knee replacement on flexion deformity. Bone Joint J. 1987;69(3):395-9.

29. Lam LO, Swift S, Shakespeare D. Fixed flexion deformity and flexion after knee arthroplasty.: What happens in the first 12 months after surgery and can a poor outcome be predicted? The Knee. 2003;10(2):181-5.

30. Lachiewicz PF, Soileau ES. Ten-year survival and clinical results of constrained components in primary total knee arthroplasty. J Arthroplasty. 2006;21(6):803-8.

31. Nikolopoulos D, Polyzois I, Magnissalis E, Bernard P, Michos I. Fracture at the stem-condylar junction of a modular femoral prosthesis in a varus-valgus constrained total knee arthroplasty. Knee Surg Sports Traumatol Arthrosc. 2012;20(6):1071-4.

32. Pang H-N, Razak HRBA, Jamiecson P, Teeter MG, Naudie DD, MacDonald SJ. Factors affecting wear of constrained polyethylene tibial inserts in total knee arthroplasty. J Arthroplasty. 2016;31(6):1340-5. 
33. Lee K, Goodman SB. Current state and future of joint replacements in the hip and knee. Expert Rev Med Devices. 2008;5(3):383-93.

34. Kurtz SM, Lau E, Ong K, Zhao K, Kelly M, Bozic KJ. Future young patient demand for primary and revision joint replacement: national projections from 2010 to 2030. Clin Orthop Relat Res. 2009;467(10):2606-12.

35. Jones DL, Bhanegaonkar AJ, Billings AA, Kriska AM, Irrgang JJ, Crossett LS, et al. Differences between actual and expected leisure activities after total knee arthroplasty for osteoarthritis. J Arthroplasty. 2012;27(7):1289-96.

36. Walldius B. Arthroplasty of the knee using an endoprosthesis. Acta Orthop Scand. 1957;28(sup24):1-112.

37. Gunston FH. Polycentric knee arthroplasty: prosthetic simulation of normal knee movement. Bone Joint J. 1971;53(2):272-7.

38. Freeman M, Swanson S, Todd R. Total replacement of the knee using the Freeman-Swanson knee prosthesis. Clin Orthop Relat Res. 1973;94:153-70.

39. Robinson RP. The early innovators of today's resurfacing condylar knees. J Arthroplasty. 2005;20:2-26.

40. Insall J, Scott WN, Ranawat CS. The total condylar knee prosthesis. A report of two hundred and twenty cases. J Bone Joint Surg Am. 1979;61(2):173-80.

41. Inglis A, Lane L, editors. Total knee replacement using the duo-patella prosthesis. 45th Annual Meeting of the American Academy of Orthopaedic Surgeons; 1978; Dallas, TX.

42. Insall JN, Lachiewicz P, Burstein A. The posterior stabilized condylar prosthesis: a modification of the total condylar design. Two to four-year clinical experience. $\mathrm{J}$ Bone Joint Surg. 1982;64(9):1317-23.

43. Indelli P, Aglietti P, Buzzi R, Baldini A. The Insall-Burstein II prosthesis: a 5-to 9-year follow-up study in osteoarthritic knees. J Arthroplasty. 2002;17(5):544-9.

44. Jacobs W, Clement DJ, Wymenga A. Retention versus sacrifice of the posterior cruciate ligament in total knee replacement for treatment of osteoarthritis and rheumatoid arthritis. Cochrane Database Syst Rev. 2005;4.

45. Verra WC, van den Boom LG, Jacobs W, Clement DJ, Wymenga AA, Nelissen RG. Retention versus sacrifice of the posterior cruciate ligament in total knee arthroplasty for treating osteoarthritis. The Cochrane Library. 2013.

46. Sorger JI, Federle D, Kirk PG, Grood E, Cochran J, Levy M. The posterior cruciate ligament in total knee arthroplasty. J Arthroplasty. 1997;12(8):869-79.

47. Malkani AL, Rand JA, Bryan RS, Wallrichs SL. Total knee arthroplasty with the kinematic condylar prosthesis. A ten-year follow-up study. J Bone Joint Surg. 1995;77(3):423-31.

48. Goodfellow J, O'Connor J. The mechanics of the knee and prosthesis design. Bone Joint J. 1978;60(3):358-69.

49. Mihalko WM, Creek AT, Mary MN, Williams JL, Komatsu DE. Mechanoreceptors found in a posterior cruciate ligament from a well-functioning total knee arthroplasty retrieval. J Arthroplasty. 2011;26(3):504. e9-. e12.

50. Conditt MA, Noble PC, Bertolusso R, Woody J, Parsley BS. The PCL significantly affects the functional outcome of total knee arthroplasty. J Arthroplasty. 2004;19(7):107-12. 
51. Wünschel M, Leasure JM, Dalheimer P, Kraft N, Wülker N, Müller O. Differences in knee joint kinematics and forces after posterior cruciate retaining and stabilized total knee arthroplasty. The Knee. 2013;20(6):416-21.

52. Fontanesi G, Rotini R, Pignedoli P, Giancecchi F. Retention of the posterior cruciate ligament in total knee arthroplasty. Ital J Orthop Traumatol. 1991;17(1):65-71.

53. Pereira DS, Jaffe FF, Ortiguera C. Posterior cruciate ligament-sparing versus posterior cruciate ligament-sacrificing arthroplasty: functional results using the same prosthesis. J Arthroplasty. 1998;13(2):138-44.

54. Scott RD, Volatile TB. Twelve years' experience with posterior cruciate-retaining total knee arthroplasty. Clin Orthop Relat Res. 1986(205):100-7.

55. Freeman M, Insall JN, Besser W, Walker PS, Hallel T. Excision of the cruciate ligaments in total knee replacement. Clin Orthop Relat Res. 1977(126):209-12.

56. Lombardi JA, Berend K. Posterior cruciate ligament-retaining, posterior stabilized, and varus/valgus posterior stabilized constrained articulations in total knee arthroplasty. Instr Course Lect. 2006;55:419-27.

57. Dennis DA, Komistek RD, Colwell Jr CE, Ranawat CS, Scott RD, Thornhill TS, et al. In vivo anteroposterior femorotibial translation of total knee arthroplasty: a multicenter analysis. Clin Orthop Relat Res. 1998;356:47-57.

58. Dennis DA, Komistek RD, Mahfouz MR, Walker SA, Tucker A. A multicenter analysis of axial femorotibial rotation after total knee arthroplasty. Clin Orthop Relat Res. 2004;428:180-9.

59. Blaha JD. The rationale for a total knee implant that confers anteroposterior stability throughout range of motion1. J Arthroplasty. 2004;19(4):22-6.

60. Stoddard JE, Deehan DJ, Bull AM, McCaskie AW, Amis AA. The kinematics and stability of single-radius versus multi-radius femoral components related to Mid-range instability after TKA. J Orthop Res. 2013;31(1):53-8.

61. Clary CW, Fitzpatrick CK, Maletsky LP, Rullkoetter PJ. The influence of total knee arthroplasty geometry on mid-flexion stability: an experimental and finite element study. J Biomech. 2013;46(7):1351-7.

62. Delport H, Banks S, De Schepper J, Bellemans J. A kinematic comparison of fixed-and mobile-bearing knee replacements. Bone Joint J. 2006;88(8):1016-21.

63. Dennis DA, Komistek RD, Mahfouz MR. In vivo fluoroscopic analysis of fixedbearing total knee replacements. Clin Orthop Relat Res. 2003;410:114-30.

64. Banks S, Bellemans J, Nozaki H, Whiteside LA, Harman M, Hodge WA. Knee motions during maximum flexion in fixed and mobile-bearing arthroplasties. Clin Orthop Relat Res. 2003;410:131-8.

65. Mahoney OM, McClung CD, dela Rosa MA, Schmalzried TP. The effect of total knee arthroplasty design on extensor mechanism function. J Arthroplasty. 2002;17(4):416-21.

66. Blunn GW, Walker PS, Joshi A, Hardinge K. The dominance of cyclic sliding in producing wear in total knee replacements. Clin Orthop Relat Res. 1991(273):253-60.

67. Hollister AM, Jatana S, Singh AK, Sullivan WW, Lupichuk AG. The axes of rotation of the knee. Clin Orthop Relat Res. 1993;290:259-68. 
68. Kessler O, Dürselen L, Banks S, Mannel H, Marin F. Sagittal curvature of total knee replacements predicts in vivo kinematics. Clin Biomech. 2007;22(1):52-8.

69. Ostermeier S, Stukenborg-Colsman C. Quadriceps force after TKA with femoral single radius: an in vitro study. Acta Orthop. 2011;82(3):339-43.

70. Shimizu N, Tomita T, Yamazaki T, Yoshikawa H, Sugamoto K. In vivo movement of femoral flexion axis of a single-radius total knee arthroplasty. $\mathrm{J}$ Arthroplasty. 2014;29(12):2407-11.

71. Li G, Most E, Otterberg E, Sabbag K, Zayontz S, Johnson T, et al. Biomechanics of posterior-substituting total knee arthroplasty: an in vitro study. Clin Orthop Relat Res. 2002;404:214-25.

72. Mihalko WM, Lowell J, Higgs G, Kurtz S. Total knee post-cam design variations and their effects on kinematics and wear patterns. Orthopedics. 2016;39(3):S45S9.

73. Moynihan AL, Varadarajan KM, Hanson GR, Park S-E, Nha KW, Suggs JF, et al. In vivo knee kinematics during high flexion after a posterior-substituting total knee arthroplasty. Int Orthop. 2010;34(4):497-503.

74. Walker PS, Lowry MT, Kumar A. The effect of geometric variations in posteriorstabilized knee designs on motion characteristics measured in a knee loading machine. Clin Orthop Relat Res. 2014;472(1):238-47.

75. Suggs JF, Hanson GR, Park SE, Moynihan AL, Li G. Patient function after a posterior stabilizing total knee arthroplasty: cam-post engagement and knee kinematics. Knee Surg Sports Traumatol Arthrosc. 2008;16(3):290-6.

76. Lin K-J, Huang C-H, Liu Y-L, Chen W-C, Chang T-W, Yang C-T, et al. Influence of post-cam design of posterior stabilized knee prosthesis on tibiofemoral motion during high knee flexion. Clin Biomech. 2011;26(8):847-52.

77. Willing R, Kim IY. Three dimensional shape optimization of total knee replacements for reduced wear. Struct Multidiscipl Optim. 2009;38(4):405-14.

78. McEwen H, Barnett P, Bell C, Farrar R, Auger D, Stone M, et al. The influence of design, materials and kinematics on the in vitro wear of total knee replacements. J Biomech. 2005;38(2):357-65.

79. Sathasivam $S$, Walker $P$. The conflicting requirements of laxity and conformity in total knee replacement. J Biomech. 1999;32(3):239-47.

80. D'lima DD, Chen PC, Colwell Jr CW. Polyethylene contact stresses, articular congruity, and knee alignment. Clin Orthop Relat Res. 2001;392:232-8.

81. Buechel FF, Pappas M. The New Jersey Low-Contact-Stress Knee Replacement System: biomechanical rationale and review of the first 123 cemented cases. Arch Orthop Trauma Surg. 1986;105(4):197-204.

82. Goodfellow JW, O'Connor J. Clinical results of the Oxford knee. Surface arthroplasty of the tibiofemoral joint with a meniscal bearing prosthesis. Clin Orthop Relat Res. 1986(205):21-42.

83. Jones V, Barton D, Fitzpatrick D, Auger D, Stone M, Fisher J. An experimental model of tibial counterface polyethylene wear in mobile bearing knees: The influence of design and kinematics. Biomed Mater Eng. 1999;9(3):189-96.

84. Komistek RD, Dennis DA, Mahfouz MR, Walker S, Outten J. In vivo polyethylene bearing mobility is maintained in posterior stabilized total knee arthroplasty. Clin Orthop Relat Res. 2004;428:207-13. 
85. D'lima DD, Trice M, Urquhart AG, Colwell Jr CW. Tibiofemoral conformity and kinematics of rotating-bearing knee prostheses. Clin Orthop Relat Res. 2001;386:235-42.

86. Walker PS, Sussman-Fort JM, Yildirim G, Boyer J. Design features of total knees for achieving normal knee motion characteristics. J Arthroplasty. 2009;24(3):47583.

87. Pajarinen J, Lin T-H, Sato T, Yao Z, Goodman S. Interaction of materials and biology in total joint replacement-successes, challenges and future directions. $\mathrm{J}$ Mater Chem B. 2014;2(41):7094-108.

88. Naudie DD, Ammeen DJ, Engh GA, Rorabeck CH. Wear and osteolysis around total knee arthroplasty. J Am Acad Orthop Surg. 2007;15(1):53-64.

89. Wasielewski RC, Parks N, Williams I, Surprenant H, Collier JP, Engh G. Tibial insert undersurface as a contributing source of polyethylene wear debris. Clin Orthop Relat Res. 1997;345:53-9.

90. White SE, Paxson RD, Tanner MG, Whiteside LA. Effects of sterilization on wear in total knee arthroplasty. Clin Orthop Relat Res. 1996;331:164-71.

91. Bell CJ, Walker PS, Abeysundera MR, Simmons JM, King PM, Blunn GW. Effect of oxidation on delamination of ultrahigh-molecular-weight polyethylene tibial components. J Arthroplasty. 1998;13(3):280-90.

92. Aglietti P, Sensi L, Cuomo P, Ciardullo A. Rotational position of femoral and tibial components in TKA using the femoral transepicondylar axis. Clin Orthop Relat Res. 2008;466(11):2751-5.

93. Luo C-F. Reference axes for reconstruction of the knee. The Knee. 2004;11(4):251-7.

94. Hungerford DS, Krackow KA. Total joint arthroplasty of the knee. Clin Orthop Relat Res. 1985(192):23-33.

95. Insall JN, Binazzi R, Soudry M, Mestriner LA. Total knee arthroplasty. Clin Orthop Relat Res. 1985(192):13-22.

96. Johnson F, Leitl S, Waugh W. The distribution of load across the knee. A comparison of static and dynamic measurements. Bone Joint J. 1980;62(3):346-9.

97. Mihalko W, Miller C, Krackow K. Total knee arthroplasty ligament balancing and gap kinematics with posterior cruciate ligament retention and sacrifice. Am J Orthop. 2000;29(8):610-6.

98. Nagamine R, White SE, McCarthy DS, Whiteside LA. Effect of rotational malposition of the femoral component on knee stability kinematics after total knee arthroplasty. J Arthroplasty. 1995;10(3):265-70.

99. Nagamine R, Whiteside LA, Otani T, White SE, McCarthy DS. Effect of medial displacement of the tibial tubercle on patellar position after rotational malposition of the femoral component in total knee arthroplasty. J Arthroplasty. 1996;11(1):104-10.

100. Berger R, Seel M, Schleiden M. Computerized tomographic determination of the normal tibiofemoral rotational angle: A guide to tibial component rotational alignment in TKA. Orthop Trans. 1993;17(4):1174-9.

101. Nicoll D, Rowley D. Internal rotational error of the tibial component is a major cause of pain after total knee replacement. Bone Joint J. 2010;92(9):1238-44. 
102. Barrack RL, Schrader T, Bertot AJ, Wolfe MW, Myers L. Component rotation and anterior knee pain after total knee arthroplasty. Clin Orthop Relat Res. 2001;392:46-55.

103. Eckhoff D, Metzger R, Vandewalle M. Malrotation associated with implant alignment technique in total knee arthroplasty. Clin Orthop Relat Res. 1995(321):28-31.

104. Akagi M, Oh M, Nonaka T, Tsujimoto H, Asano T, Hamanishi C. An anteroposterior axis of the tibia for total knee arthroplasty. Clin Orthop Relat Res. 2004;420:213-9.

105. Bindelglass DF. Rotational alignment of the tibial component in total knee arthroplasty. Orthopedics. 2001;24(11):1049-52.

106. Rossi R, Bruzzone M, Bonasia DE, Marmotti A, Castoldi F. Evaluation of tibial rotational alignment in total knee arthroplasty: a cadaver study. Knee Surg Sports Traumatol Arthrosc. 2010;18(7):889-93.

107. Indelli PF, Graceffa A, Marcucci M, Baldini A. Rotational alignment of the tibial component in total knee arthroplasty. Ann Transl Med. 2016;4(1).

108. Lee D-H, Seo J-G, Moon Y-W. Synchronisation of tibial rotational alignment with femoral component in total knee arthroplasty. Int Orthop. 2008;32(2):223-7.

109. Puloski S, McCalden R, MacDonald S, Rorabeck C, Bourne R. Tibial post wear in posterior stabilized total knee arthroplasty. J Bone Joint Surg Am. 2001;83(3):390-.

110. Ikeuchi M, Yamanaka N, Okanoue Y, Ueta E, Tani T. Determining the rotational alignment of the tibial component at total knee replacement: a comparison of two techniques. Bone Joint J. 2007;89(1):45-9.

111. Howell S, Kuznik K, Hull M, Siston R. Results of an initial experience with custom-fit positioning total knee arthroplasty in a series of 48 patients. Orthopedics. 2008;31(9):857.

112. Howell SM, Hull ML. Kinematic alignment in total knee arthroplasty. Insall and Scott Surgery of the Knee. Philadelphia, PA: Elsevier; 2012. p. 1255-68.

113. Ishikawa M, Kuriyama S, Ito H, Furu M, Nakamura S, Matsuda S. Kinematic alignment produces near-normal knee motion but increases contact stress after total knee arthroplasty: a case study on a single implant design. The Knee. 2015;22(3):206-12.

114. Mihalko WM, Woodard EL, Hebert CT, Crockarell JR, Williams JL. Biomechanical validation of medial pie-crusting for soft-tissue balancing in knee arthroplasty. J Arthroplasty. 2015;30(2):296-9.

115. Mihalko WM, Saleh KJ, Krackow KA, Whiteside LA. Soft-tissue balancing during total knee arthroplasty in the varus knee. J Am Acad Orthop Surg. 2009; 17(12):766-74.

116. Pitta M, Esposito CI, Li Z, Lee Y-y, Wright TM, Padgett DE. Failure after modern total knee arthroplasty: a prospective study of 18,065 knees. J Arthroplasty 2017.

117. Massin P. How does total knee replacement technique influence polyethylene wear? Orthop Traumatol Surg Res. 2017;103(1):S21-S7.

118. Mitchelson AJ, Wilson CJ, Mihalko WM, Grupp TM, Manning BT, Dennis DA, et al. Biomaterial hypersensitivity: is it real? Supportive evidence and approach 
considerations for metal allergic patients following total knee arthroplasty.

Biomed Res Int. 2015;2015.

119. Callaghan J, O'rourke M, Saleh K. Why knees fail: lessons learned. J

Arthroplasty. 2004;19(4 Suppl 1):31.

120. Lim H-A, Song E-K, Seon J-K, Park K-S, Shin Y-J, Yang H-Y. Causes of aseptic persistent pain after total knee arthroplasty. Clin Orthop Surg. 2017;9(1):50-6.

121. Le DH, Goodman SB, Maloney WJ, Huddleston JI. Current modes of failure in TKA: infection, instability, and stiffness predominate. Clin Orthop Relat Res. 2014;472(7):2197-200.

122. Manning B, Lewis N, Tzeng T, Saleh J, Potty A, Dennis D, et al. Diagnosis and management of extra-articular causes of pain after total knee arthroplasty. Instr Course Lect. 2015;64:381-8.

123. Kawanabe K, Clarke IC, Tamura J, Akagi M, Good VD, Williams PA, et al. Effects of A-P translation and rotation on the wear of UHMWPE in a total knee joint simulator. J Biomed Mater Res A. 2001;54(3):400-6.

124. Kretzer JP, Jakubowitz E, Sonntag R, Hofmann K, Heisel C, Thomsen M. Effect of joint laxity on polyethylene wear in total knee replacement. J Biomech. 2010;43(6):1092-6.

125. McEwen H, Fisher J, Goldsmith A, Auger D, Hardaker C, Stone M. Wear of fixed bearing and rotating platform mobile bearing knees subjected to high levels of internal and external tibial rotation. J Mater Sci Mater Med. 2001;12(1012):1049-52.

126. Barnett PI, Fisher J, Auger DD, Stone MH, Ingham E. Comparison of wear in a total knee replacement under different kinematic conditions. J Mater Sci Mater Med. 2001;12(10):1039-42.

127. Malo M, Vince KG. The unstable patella after total knee arthroplasty: etiology, prevention, and management. J Am Acad Orthop Surg. 2003;11(5):364-71.

128. Husted H, Jensen TT. Influence of the pneumatic tourniquet on patella tracking in total knee arthroplasty: a prospective randomized study in 100 patients. J Arthroplasty. 2005;20(6):694-7.

129. Marson BM, Tokish J. The effect of a tourniquet on intraoperative patellofemoral tracking during total knee arthroplasty. J Arthroplasty. 1999;14(2):197-9.

130. Laskin RS. Lateral release rates after total knee arthroplasty. Clin Orthop Relat Res. 2001;392:88-93.

131. Hallab NJ, Jacobs JJ. Biologic effects of implant debris. Bull Hop Jt Dis. 2009;67(2):182.

132. Goodman SB. Wear particles, periprosthetic osteolysis and the immune system. Biomaterials. 2007;28(34):5044-8.

133. Nich C, Takakubo Y, Pajarinen J, Gallo J, Konttinen YT, Takagi M, et al. The Role of Macrophages in the Biological Reaction to Wear Debris from Artificial Joints. J Long Term Eff Med Implants. 2016;26(4).

134. Caicedo MS, Desai R, McAllister K, Reddy A, Jacobs JJ, Hallab NJ. Soluble and particulate Co-Cr-Mo alloy implant metals activate the inflammasome danger signaling pathway in human macrophages: A novel mechanism for implant debris reactivity. J Orthop Res. 2009;27(7):847-54. 
135. Greenfield EM. Do genetic susceptibility, Toll-like receptors, and pathogenassociated molecular patterns modulate the effects of wear? Clin Orthop Relat Res. 2014;472(12):3709-17.

136. Hirayama T, Tamaki Y, Takakubo Y, Iwazaki K, Sasaki K, Ogino T, et al. Tolllike receptors and their adaptors are regulated in macrophages after phagocytosis of lipopolysaccharide-coated titanium particles. J Orthop Res. 2011;29(7):984-92.

137. Gu Q, Shi Q, Yang H. The role of TLR and chemokine in wear particle-induced aseptic loosening. Biomed Res Int. 2012;2012.

138. Gallo J, Goodman SB, Konttinen YT, Wimmer MA, Holinka M. Osteolysis around total knee arthroplasty: a review of pathogenetic mechanisms. Acta Biomater 2013;9(9):8046-58.

139. Malik MH, Bayat A, Jury F, Ollier WE, Kay PR. Genetic susceptibility to hip arthroplasty failure - association with the RANK/OPG pathway. Int Orthop. 2006;30(3):177-81.

140. Hallab N, Merritt K, Jacobs JJ. Metal sensitivity in patients with orthopaedic implants. J Bone Joint Surg Am. 2001;83(3):428-.

141. Granchi D, Ciapetti G, Savarino L, Stea S, Filippini F, Sudanese A, et al. Expression of the CD69 activation antigen on lymphocytes of patients with hip prosthesis. Biomaterials. 2000;21(20):2059-65.

142. Gibon E, Amanatullah DF, Loi F, Pajarinen J, Nabeshima A, Yao Z, et al. The biological response to orthopaedic implants for joint replacement: Part I: Metals. J Biomed Mater Res Part B. 2017;105(7):2162-73.

143. Eltit F, Assiri A, Garbuz D, Duncan C, Masri B, Greidanus N, et al. Adverse reactions to metal on polyethylene implants: Highly destructive lesions related to elevated concentration of cobalt and chromium in synovial fluid. J Biomed Mater Res A. 2017;105(7):1876-86.

144. Gilbert JL, Sivan S, Liu Y, Kocagöz SB, Arnholt CM, Kurtz SM. Direct in vivo inflammatory cell-induced corrosion of CoCrMo alloy orthopedic implant surfaces. J Biomed Mater Res A. 2015;103(1):211-23.

145. Cerquiglini A, Henckel J, Hothi HS, Di Laura A, Skinner JA, Hart AJ. Inflammatory cell-induced corrosion in total knee arthroplasty: a retrieval study. J Biomed Mater Res B. 2018;106(1):460-7.

146. Di Laura A, Hothi HS, Meswania JM, Whittaker RK, de Villiers D, Zustin J, et al. Clinical relevance of corrosion patterns attributed to inflammatory cell-induced corrosion: A retrieval study. J Biomed Mater Res B. 2017;105(1):155-64.

147. Hallab NJ, Vermes C, Messina C, Roebuck KA, Glant TT, Jacobs JJ. Concentration-and composition-dependent effects of metal ions on human MG-63 osteoblasts. J Biomed Mater Res A. 2002;60(3):420-33.

148. Gupta R, Phan D, Schwarzkopf R. Total knee arthroplasty failure induced by metal hypersensitivity. Am J Case Rep. 2015;16:542.

149. Fehring KA, Fehring TK. Modes of failure in metal-on-metal total hip arthroplasty. Orthop Clin North Am. 2015;46(2):185-92.

150. Yao JJ, Lewallen EA, Trousdale WH, Xu W, Thaler R, Salib CG, et al. Local cellular responses to titanium dioxide from orthopedic implants. Biores Open Access. 2017;6(1):94-103. 
151. Willis-Owen C, Keene G, Oakeshott R. Early metallosis-related failure after total knee replacement. Bone Joint J. 2011;93(2):205-9.

152. Oliveira CA, Candelária IS, Oliveira PB, Figueiredo A, Caseiro-Alves F. Metallosis: a diagnosis not only in patients with metal-on-metal prostheses. Eur J Radiol Open. 2015;2:3-6.

153. Haddad F, Thakrar R, Hart A, Skinner J, Nargol A, Nolan J, et al. Metal-on-metal bearings. J Bone Joint Surg Br. 2011;93(5):572-9.

154. Campbell P, Ebramzadeh E, Nelson S, Takamura K, De Smet K, Amstutz HC. Histological features of pseudotumor-like tissues from metal-on-metal hips. Clin Orthop Relat Res. 2010;468(9):2321-7.

155. Whitehouse M, Endo M, Zachara S, Nielsen T, Greidanus N, Masri B, et al. Adverse local tissue reactions in metal-on-polyethylene total hip arthroplasty due to trunnion corrosion. Bone Joint J. 2015;97(8):1024-30.

156. Lons A, Putman S, Pasquier G, Migaud H, Drumez E, Girard J. Metallic ion release after knee prosthesis implantation: a prospective study. Int Orthop. 2017:1-6.

157. Savarino L, Tigani D, Greco M, Baldini N, Giunti A. The potential role of metal ion release as a marker of loosening in patients with total knee replacement. Bone Joint J. 2010;92(5):634-8.

158. Lützner J, Hartmann A, Dinnebier G, Spornraft-Ragaller P, Hamann C, Kirschner S. Metal hypersensitivity and metal ion levels in patients with coated or uncoated total knee arthroplasty: a randomised controlled study. Int Orthop. 2013;37(10):1925-31.

159. IARC Monographs on the Evaluation of Carcinogenic Risks to Humans. International Agency for Research of Cancer. 1997;49:17-33.

160. Willert H-G, Buchhorn GH, Fayyazi A, Flury R, Windler M, Köster G, et al. Metal-on-metal bearings and hypersensitivity in patients with artificial hip joints: a clinical and histomorphological study. JBJS. 2005;87(1):28-36.

161. Nam D, Li K, Riegler V, Barrack RL. Patient-reported metal allergy: a risk factor for poor outcomes after total joint arthroplasty? J Arthroplasty 2016;31(9):1910-5.

162. Cooper HJ, Della Valle CJ, Berger RA, Tetreault M, Paprosky WG, Sporer SM, et al. Corrosion at the head-neck taper as a cause for adverse local tissue reactions after total hip arthroplasty. J Bone Joint Surg Am. 2012;94(18):1655.

163. Plummer DR, Berger RA, Paprosky WG, Sporer SM, Jacobs JJ, Della Valle CJ. Diagnosis and management of adverse local tissue reactions secondary to corrosion at the head-neck junction in patients with metal on polyethylene bearings. J Arthroplasty. 2016;31(1):264-8.

164. McGrory BJ, MacKenzie J, Babikian G. A high prevalence of corrosion at the head-neck taper with contemporary Zimmer non-cemented femoral hip components. J Arthroplasty. 2015;30(7):1265-8.

165. Swiatkowska I, Mosselmans JFW, Geraki T, Wyles CC, Maleszewski JJ, Henckel J, et al. Synchrotron Analysis of Human Organ Tissue Exposed to Implant Material. J Trace Elem Med Biol. 2017.

166. Kaufman AM, Alabre CI, Rubash HE, Shanbhag AS. Human macrophage response to UHMWPE, TiAlV, CoCr, and alumina particles: analysis of multiple cytokines using protein arrays. J Biomed Mater Res Part A. 2008;84(2):464-74. 
167. Savarino L, Tigani D, Greco M, Baldini N, Giunti A. The potential role of metal ion release as a marker of loosening in patients with total knee replacement. Bone \& Joint Journal. 2010;92(5):634-8.

168. Loria MP, Dambra P, Moretti B, Patella V, Capuzzimati L, Cavallo E, et al. Role of cytokines in gonarthrosis and knee prosthesis aseptic loosening. J Orthop Sci. 2004;9(3):274-9.

169. Bertolini DR, Nedwin GE, Bringman TS, Smith DD, Mundy GR. Stimulation of bone resorption and inhibition of bone formation in vitro by human tumour necrosis factors. Nature. 1986.

170. Gowen M, Wood DD, Ihrie EJ, McGuire MK, Russell RGG. An interleukin 1 like factor stimulates bone resorption in vitro. Nature. 1983;306(5941):378-80.

171. Haynes D, Crotti T, Zreiqat H. Regulation of osteoclast activity in peri-implant tissues. Biomaterials. 2004;25(20):4877-85.

172. Roodman G. Perspectives: Interleukin-6: An osteotropic factor? J Bone Miner Res. 1992;7(5):475-8.

173. Blaine TA, Rosier RN, Puzas JE, Looney RJ, Reynolds PR, Reynolds SD, et al. Increased levels of tumor necrosis factor- $\alpha$ and interleukin- 6 protein and messenger RNA in human peripheral blood monocytes due to titanium particles. J Bone Joint Surg. 1996;78(8):1181-92.

174. Purdue PE, Koulouvaris P, Potter HG, Nestor BJ, Sculco TP. The cellular and molecular biology of periprosthetic osteolysis. Clin Orthop Relat Res. 2007;454:251-61.

175. Haleem-Smith H, Argintar E, Bush C, Hampton D, Postma WF, Chen FH, et al. Biological responses of human mesenchymal stem cells to titanium wear debris particles. J Orthop Res. 2012;30(6):853-63.

176. Nakashima Y, Sun D-H, Trindade M, Chun L, Song Y, Goodman S, et al. Induction of macrophage $\mathrm{CC}$ chemokine expression by titanium alloy and bone cement particles. J Bone Joint Surg Br. 1999;81(1):155-62.

177. Dasa V, Kramer JM, Gaffen SL, Kirkwood KL, Mihalko WM. Is monocyte chemotactic protein 1 elevated in aseptic loosening of TKA?: a pilot study. Clin Orthop Relat Res. 2012;470(7):1879-84.

178. Ramos CD, Canetti C, Souto JT, Silva JS, Hogaboam CM, Ferreira SH, et al. MIP-1 $\alpha$ [CCL3] acting on the CCR1 receptor mediates neutrophil migration in immune inflammation via sequential release of TNF- $\alpha$ and LTB4. J Leukoc Biol. 2005;78(1):167-77.

179. Al-Saffar N, Khwaja HA, Kadoya Y, Revell PA. Assessment of the role of GMCSF in the cellular transformation and the development of erosive lesions around orthopaedic implants. Am J Clin Pathol. 1996;105(5):628-39.

180. Fleetwood AJ, Lawrence T, Hamilton JA, Cook AD. Granulocyte-macrophage colony-stimulating factor (CSF) and macrophage CSF-dependent macrophage phenotypes display differences in cytokine profiles and transcription factor activities: implications for CSF blockade in inflammation. J Immunol. 2007;178(8):5245-52.

181. Jämsen E, Kouri VP, Olkkonen J, Cör A, Goodman SB, Konttinen YT, et al. Characterization of macrophage polarizing cytokines in the aseptic loosening of total hip replacements. J Orthop Res. 2014;32(9):1241-6. 
182. Li T-F, Santavirta S, Waris V, Lassus J, Lindroos L, Xu J-W, et al. No lymphokines in T-cells around loosened hip prostheses. Acta Orthop Scand. 2001;72(3):241-7.

183. Hercus B, Revell PA. Phenotypic characteristics of T lymphocytes in the interfacial tissue of aseptically loosened prosthetic joints. J Mater Sci Mater Med. 2001;12(10):1063-7.

184. Woodard EL. Total knee arthroplasty wear is caused by malrotation and excessive laxity [Theses and Dissertations (ETD)]. Memphis, TN: University of Tennessee Health Science Center; 2014.

185. Woodard E, Williams J, Mihalko W. Laxity differences in cruciate-retaining and posterior-stabilized total knee arthroplasty. Bone Joint J. 2016;98(SUPP 4):127-.

186. Mihalko WM, Whiteside LA, Krackow KA. Comparison of ligament-balancing techniques during total knee arthroplasty. J Bone Joint Surg. 2003;85:132-5.

187. Saeki K, Mihalko WM, Patel V, Conway J, Naito M, Thrum H, et al. Stability after medial collateral ligament release in total knee arthroplasty. Clin Orthop Relat Res. 2001;392:184-9.

188. Jojima H, Whiteside LA, Ogata K. Effect of tibial slope or posterior cruciate ligament release on knee kinematics. Clin Orthop Relat Res. 2004;426:194-8.

189. Kanamiya T, Whiteside LA, Nakamura T, Mihalko WM, Steiger J, Naito M. Effect of Selective Lateral Ligament Release on Stability in Knee Arthroplasty. Clin Orthop Relat Res. 2002;404:24-31.

190. Connor JM. Experimental Kinematic Analysis of Cadaver Knees Using a Knee Simulator and Surgical Navigation System. Memphis, TN: University of Memphis; 2011.

191. Knox DA. Correlation Between Computed Contact Parameters and Wear Patterns on a Retrieved UHMWPE Tibial Insert. Memphis, TN: University of Memphis; 2011.

192. Conner DJ. Using Computer Assisted Tomography to Acquire More Detailed Subject Specific Information for Kinematic Analysis. Memphis, TN: University of Memphis; 2012.

193. Lindsey JA. Utilizing a Computer Assisted Surgery System to Output Kinematic Measures for a Simulated Single Leg Lunge for Total Knee Arthroplasty. Memphis, TN: University of Memphis; 2016.

194. ASTM F561-13. Standard Practice for Retrieval and Analysis of Medical Devices, and Associated Tissues and Fluids. ASTM International: West Conshohocken, PA; 2013.

195. Hood RW, Wright TM, Burstein AH. Retrieval analysis of total knee prostheses: a method and its application to 48 total condylar prostheses. J Biomed Mater Res A. 1983;17(5):829-42.

196. Arnholt CM, MacDonald DW, Malkani AL, Klein GR, Rimnac CM, Kurtz SM, et al. Corrosion damage and wear mechanisms in long-term retrieved $\mathrm{CoCr}$ femoral components for total knee arthroplasty. J Arthroplasty. 2016;31(12):2900-6.

197. Fehring TK, McAvoy G. Fluoroscopic evaluation of the painful total knee arthroplasty. Clin Orthop Relat Res. 1996;331:226-33. 
198. Mintz AD, Pilkington C, Howie D. A comparison of plain and fluoroscopically guided radiographs in the assessment of arthroplasty of the knee. J Bone Joint Surg Am. 1989;71(9):1343-7.

199. Ecker ML, Lotke PA, Windsor RE, Cella JP. Long-term results after total condylar knee arthroplasty. Significance of radiolucent lines. Clin Orthop Relat Res. 1987(216):151-8.

200. Vyskocil P, Gerber C, Bamert P. Radiolucent lines and component stability in knee arthroplasty: standard versus fluoroscopically-assisted radiographs. J Bone Joint Surg Br. 1999;81(1):24-6.

201. Kerger BD, Gerads R, Gurleyuk H, Urban A, Paustenbach DJ. Total cobalt determination in human blood and synovial fluid using inductively coupled plasma-mass spectrometry: method validation and evaluation of performance variables affecting metal hip implant patient samples. Toxicol Environ Chem. 2015;97(9):1145-63.

202. Jacobs JJ, Roebuck KA, Archibeck M, Hallab NJ, Glant TT. Osteolysis: basic science. Clin Orthop Relat Res. 2001;393:71-7.

203. Kovacik MW, Gradisar Jr IA, Haprian JJ, Alexander TS. Osteolytic indicators found in total knee arthroplasty synovial fluid aspirates. Clin Orthop Relat Res. 2000;379:186-94.

204. Kurtz SM. UHMWPE biomaterials handbook: ultra high molecular weight polyethylene in total joint replacement and medical devices: Academic Press; 2009.

205. Medel FJ, Kurtz SM, Sharkey PF, Austin MS, Klein GR, Cohen AR, et al. Post damage in contemporary posterior-stabilized tibial inserts: Influence of implant design and clinical relevance. J Arthroplasty. 2011;26(4):606-14.

206. Conditt MA, Thompson MT, Usrey MM, Ismaily SK, Noble PC. Backside wear of polyethylene tibial inserts: mechanism and magnitude of material loss. J Bone Joint Surg. 2005;87(2):326-31.

207. Wright T, Bartel D. The problem of surface damage in polyethylene total knee components. Clin Orthop Relat Res. 1986(205):67-74.

208. Wasielewski RC. The causes of insert backside wear in total knee arthroplasty. Clin Orthop Relat Res. 2002;404:232-46.

209. Wasielewski RC, Galante JO, Leighty RM, Natarajan RN, Rosenberg AG. Wear patterns on retrieved polyethylene tibial inserts and their relationship to technical considerations during total knee arthroplasty. Clin Orthop Relat Res. 1994(299):31-43.

210. Surace MF, Berzins A, Urban RM, Jacobs JJ, Berger RA, Natarajan RN, et al. Backsurface wear and deformation in polyethylene tibial inserts retrieved postmortem. Clin Orthop Relat Res. 2002;404:14-23.

211. Teeter MG, Lanting BA, Shrestha KR, Howard JL, Vasarhelyi EM. Contribution of surface polishing and sterilization method to backside wear in total knee arthroplasty. J Arthroplasty. 2015;30(12):2320-2.

212. Medel FJ, Kurtz SM, Hozack WJ, Parvizi J, Purtill JJ, Sharkey PF, et al. Gamma inert sterilization: a solution to polyethylene oxidation? J Bone Joint Surg Am. 2009;91(4):839. 
213. Currier BH, Currier JH, Collier JP, Mayor MB, Scott RD. Shelf life and in vivo duration. Impacts on performance of tibial bearings. Clin Orthop Relat Res. 1997(342):111-22.

214. Premnath V, Harris W, Jasty M, Merrill E. Gamma sterilization of UHMWPE articular implants: an analysis of the oxidation problem. Biomaterials. 1996;17(18):1741-53.

215. Collier JP, Sperling DK, Currier JH, Sutula LC, Saum KA, Mayor MB. Impact of gamma sterilization on clinical performance of polyethylene in the knee. $\mathrm{J}$ Arthroplasty. 1996;11(4):377-89.

216. Medel FJ, Kurtz SM, Parvizi J, Klein GR, Kraay MJ, Rimnac CM. In vivo oxidation contributes to delamination but not pitting in polyethylene components for total knee arthroplasty. J Arthroplasty. 2011;26(5):802-10.

217. Fisher J, McEwen H, Barnett P, Bell C, Stewart T, Stone M, et al. Wear of polyethylene in artificial knee joints. Current Orthopaedics. 2001;15(6):399-405.

218. Lu Y-C, Huang C-H, Chang T-K, Ho F-Y, Cheng C-K. Wear-pattern analysis in retrieved tibial inserts of mobile-bearing and fixed-bearing total knee prostheses. Bone Joint J. 2010;92(4):500-7.

219. Li S, Scuderi G, Furman B, Bhattacharyya S, Schmieg J, Insall J. Assessment of backside wear from the analysis of 55 retrieved tibial inserts. Clin Orthop Relat Res. 2002;404:75-82.

220. Griffin FM, Scuderi GR, Gillis AM, Li S, Jimenez E, Smith T. Osteolysis associated with cemented total knee arthroplasty. J Arthroplasty. 1998;13(5):5928.

221. Engh GA, Parks NL, Ammeen DJ. Tibial osteolysis in cementless total knee arthroplasty. A review of 25 cases treated with and without tibial component revision. Clin Orthop Relat Res. 1994(309):33-43.

222. Billi F, Sangiorgio SN, Aust S, Ebramzadeh E. Material and surface factors influencing backside fretting wear in total knee replacement tibial components. J Biomech 2010;43(7):1310-5.

223. Abdel MP, Gesell MW, Hoedt CW, Meyers KN, Wright TM, Haas SB. Polished trays reduce backside wear independent of post location in posterior-stabilized TKAs. Clin Orthop Relat Res. 2014;472(8):2477-82.

224. Engh GA, Lounici S, Rao AR, Collier MB. In vivo deterioration of tibial baseplate locking mechanisms in contemporary modular total knee components. J Bone Joint Surg. 2001;83(11):1660-5.

225. Levine RA, Lewicki KA, Currier JH, Mayor MB, Van Citters DW. Contribution of micro-motion to backside wear in a fixed bearing total knee arthroplasty. J Orthop Res. 2016;34(11):1933-40.

226. Callaghan JJ, O'Rourke MR, Goetz DD, Schmalzried TP, Campbell PA, Johnston RC. Tibial post impingement in posterior-stabilized total knee arthroplasty. Clin Orthop Relat Res. 2002;404:83-8.

227. Furman BD, Lipman J, Kligman M, Wright TM, Haas SB. Tibial post wear in posterior-stabilized knee replacements is design-dependent. Clin Orthop Relat Res. 2008;466(11):2650-5.

228. Banks SA, Harman MK, Hodge WA. Mechanism of anterior impingement damage in total knee arthroplasty. J Bone Joint Surg. 2002;84(suppl_2):S37-S42. 
229. Que L, Topoleski L, Parks NL. Surface roughness of retrieved CoCrMo alloy femoral components from PCA artificial total knee joints. J Biomed Mater Res A. 2000;53(1):111-8.

230. Siddique M, Rao M, Deehan D, Pinder I. Role of abrasion of the femoral component in revision knee arthroplasty. Bone Joint J. 2003;85(3):393-8.

231. Muratoglu OK, Burroughs BR, Bragdon CR, Christensen S, Lozynsky A, Harris WH. Knee simulator wear of polyethylene tibias articulating against explanted rough femoral components. Clin Orthop Relat Res. 2004;428:108-13.

232. Kretzer JP, Reinders J, Sonntag R, Hagmann S, Streit M, Jeager S, et al. Wear in total knee arthroplasty_ just a question of polyethylene? Int Orthop. 2014;38(2):335-40.

233. Heisel C, Streich N, Krachler M, Jakubowitz E, Kretzer JP. Characterization of the running-in period in total hip resurfacing arthroplasty: an in vivo and in vitro metal ion analysis. J Bone Joint Surg. 2008;90(Supplement_3):125-33.

234. Gilbert JL, Buckley CA, Jacobs JJ. In vivo corrosion of modular hip prosthesis components in mixed and similar metal combinations. The effect of crevice, stress, motion, and alloy coupling. J Biomed Mater Res A. 1993;27(12):1533-44.

235. Luetzner J, Krummenauer F, Lengel AM, Ziegler J, Witzleb W-C. Serum metal ion exposure after total knee arthroplasty. Clin Orthop Relat Res. 2007;461:13642.

236. Cracchiolo A, Revell P. Metal concentration in synovial fluids of patients with prosthetic knee arthroplasty. Clin Orthop Relat Res. 1982(170):169-74.

237. Milošev I, Levašič V, Vidmar J, Kovač S, Trebše R. pH and metal concentration of synovial fluid of osteoarthritic joints and joints with metal replacements. J Biomed Mater Res B. 2017;105(8):2507-15.

238. Jacobs JJ, Silverton C, Hallab NJ, Skipor AK, Patterson L, Black J, et al. Metal release and excretion from cementless titanium alloy total knee replacements. Clin Orthop Relat Res. 1999(358):173-80.

239. Witzleb W-C, Ziegler J, Krummenauer F, Neumeister V, Guenther K-P. Exposure to chromium, cobalt and molybdenum from metal-on-metal total hip replacement and hip resurfacing arthroplasty. Acta Orthop. 2006;77(5):697-705.

240. Ichinose S, Muneta T, Sekiya I, Itoh S, Aoki H, Tagami M. The study of metal ion release and cytotoxicity in $\mathrm{Co}-\mathrm{Cr}-\mathrm{Mo}$ and $\mathrm{Ti}-\mathrm{Al}-\mathrm{V}$ alloy in total knee prosthesis-scanning electron microscopic observation. J Mater Sci Mater Med. 2003;14(1):79-86.

241. Woodman J, Black J, Jiminez S. Isolation of serum protein organometallic corrosion products from 316LSS and HS-21 in vitro and in vivo. J Biomed Mater Res A. 1984;18(1):99-114.

242. Merritt K, Brown SA. Distribution of cobalt chromium wear and corrosion products and biologic reactions. Clin Orthop Relat Res. 1996;329:S233-S43.

243. Mabey T, Honsawek S, Tanavalee A, Yuktanandana P, Wilairatana V, Poovorawan Y. Plasma and synovial fluid inflammatory cytokine profiles in primary knee osteoarthritis. Biomarkers. 2016;21(7):639-44.

244. Perkins M, Lowell J, Kerkhof A, Mihalko W. Inflammatory Cytokines as Potential Biomarkers for Damage in Total Knee Arthroplasty. In: Greenwald A, Kurtz S, Lemons J, Mihalko W, editors. STP1606 Symposium on Beyond the 
Implant: Retrieval Analysis Methods for Implant Surveillance. West Conshohocken, PA: ASTM International; 2018.

245. Kahle P, Saal J, Schaudt K, Zacher J, Fritz P, Pawelec G. Determination of cytokines in synovial fluids: correlation with diagnosis and histomorphological characteristics of synovial tissue. Ann Rheum Dis. 1992;51(6):731.

246. Steiner G, Tohidast-Akrad M, Witzmann G, Vesely M, Studnicka-Benke A, Gal A, et al. Cytokine production by synovial $\mathrm{T}$ cells in rheumatoid arthritis. Rheumatol. 1999;38(3):202-13.

247. Richette P, Francois M, Vicaut E, Fitting C, Bardin T, Corvol M, et al. A high interleukin 1 receptor antagonist/IL-1 $\beta$ ratio occurs naturally in knee osteoarthritis. J Rheumatol. 2008;35(8):1650-4.

248. Beekhuizen M, Gierman L, Van Spil W, Van Osch G, Huizinga T, Saris DB, et al. An explorative study comparing levels of soluble mediators in control and osteoarthritic synovial fluid. Osteoarthritis Cartilage. 2013;21(7):918-22.

249. Ruth JH, Shahrara S, Park CC, Morel JC, Kumar P, Qin S, et al. Role of macrophage inflammatory protein-3 $\alpha$ and its ligand CCR6 in rheumatoid arthritis. Lab Invest. 2003;83(4):579.

250. Di Cesare PE, Chang E, Preston CF, Liu C-j. Serum interleukin-6 as a marker of periprosthetic infection following total hip and knee arthroplasty. J Bone Joint Surg. 2005;87(9):1921-7.

251. Bottner F, Wegner A, Winkelmann W, Becker K, Erren M, Götze C. Interleukin6 , procalcitonin and TNF- $\alpha$ : markers of peri-prosthetic infection following total joint replacement. Bone Joint J. 2007;89(1):94-9.

252. Randau TM, Friedrich MJ, Wimmer MD, Reichert B, Kuberra D, Stoffel-Wagner B, et al. Interleukin-6 in serum and in synovial fluid enhances the differentiation between periprosthetic joint infection and aseptic loosening. PLoS One. 2014;9(2):e89045.

253. Kishimoto T, Tanaka T. Interleukin 6. Encyclopedia of Inflammatory Diseases. 2015:1-8.

254. Liao F, Rabin RL, Smith CS, Sharma G, Nutman TB, Farber JM. CC-chemokine receptor 6 is expressed on diverse memory subsets of $T$ cells and determines responsiveness to macrophage inflammatory protein $3 \alpha$. J Immunol. 1999;162(1):186-94.

255. Martinez FO, Gordon S. The M1 and M2 paradigm of macrophage activation: time for reassessment. F1000Prime Rep. 2014;6.

256. Deirmengian C, Hallab N, Tarabishy A, Della Valle C, Jacobs JJ, Lonner J, et al. Synovial fluid biomarkers for periprosthetic infection. Clin Orthop Relat Res. 2010;468(8):2017-23.

257. Streich NA, Breusch SJ, Schneider U. Serum levels of interleukin 6 (IL-6), granulocyte-macrophage colony-stimulating factor (GM-CSF) and elastase in aseptic prosthetic loosening. Int Orthop. 2003;27(5):267-71.

258. Xu J-W, Konttinen YT, Waris V, Pätiälä H, Sorsa T, Santavirta S. Macrophagecolony stimulating factor (M-CSF) is increased in the synovial-like membrane of the periprosthetic tissues in the aseptic loosening of total hip replacement (THR). Clin Rheumatol. 1997;16(3):243-8. 
259. Takei I, Takagi M, Ida H, Ogino T, Santavirta S, Konttinen YT. High macrophage-colony stimulating factor levels in synovial fluid of loose artificial hip joints. J Rheumatol. 2000;27(4):894-9.

260. Aziz N, Nishanian P, Mitsuyasu R, Detels R, Fahey JL. Variables that affect assays for plasma cytokines and soluble activation markers. Clin Diagn Lab Immunol. 1999;6(1):89-95.

261. Aziz N, Detels R, Quint JJ, Li Q, Gjertson D, Butch AW. Stability of cytokines, chemokines and soluble activation markers in unprocessed blood stored under different conditions. Cytokine. 2016;84:17-24.

262. Zhou X, Fragala MS, McElhaney JE, Kuchel GA. Conceptual and methodological issues relevant to cytokine and inflammatory marker measurements in clinical research. Curr Opin Clin Nutr Metab Care. 2010;13(5):541.

263. Panicker G, Meadows KS, Lee DR, Nisenbaum R, Unger ER. Effect of storage temperatures on the stability of cytokines in cervical mucous. Cytokine. 2007;37(2):176-9.

264. Exley AR, Cohen J. Optimal collection of blood samples for the measurement of tumor necrosis factor $\alpha$. Cytokine. 1990;2(5):353-6.

265. Riches P, Gooding R, Millar BC, Rowbottom AW. Influence of collection and separation of blood samples on plasma IL-1, IL-6 and TNF- $\alpha$ concentrations. J Immunol Methods. 1992;153(1-2):125-31.

266. Thavasu P, Longhurst S, Joel S, Slevin M, Balkwill F. Measuring cytokine levels in blood. Importance of anticoagulants, processing, and storage conditions. J Immunol Methods. 1992;153(1-2):115-24.

267. Flower L, Ahuja RH, Humphries SE, Mohamed-Ali V. Effects of sample handling on the stability of interleukin 6 , tumour necrosis factor- $\alpha$ and leptin. Cytokine. 2000;12(11):1712-6.

268. Vgontzas AN, Bixler EO, Lin H-M, Prolo P, Trakada G, Chrousos GP. IL-6 and its circadian secretion in humans. Neuroimmunomodulation. 2005;12(3):131-40.

269. Scheff JD, Calvano SE, Lowry SF, Androulakis IP. Modeling the influence of circadian rhythms on the acute inflammatory response. J Theor Biol. 2010;264(3):1068-76.

270. Petrovsky N, McNair P, Harrison LC. Diurnal rhythms of pro-inflammatory cytokines: regulation by plasma cortisol and therapeutic implications. Cytokine. 1998;10(4):307-12.

271. Banks SA, Hodge WA. Implant design affects knee arthroplasty kinematics during stair-stepping. Clin Orthop Relat Res. 2004;426:187-93.

272. Sharkey PF, Hozack WJ, Rothman RH, Shastri S, Jacoby SM. Why are total knee arthroplasties failing today? Clin Orthop Relat Res. 2002;404:7-13.

273. Bostrom MP, Bennett AP, Rimnac CM, Wright TM. The natural history of ultra high molecular weight polyethylene. Clin Orthop Relat Res. 1994(309):20-8.

274. Kurtz S, Bartel D, Rimnac C. Postirradiation aging affects stress and strain in polyethylene components. Clin Orthop Relat Res. 1998(350):209-20.

275. Sharkey PF, Lichstein PM, Shen C, Tokarski AT, Parvizi J. Why are total knee arthroplasties failing today - has anything changed after 10 years? J Arthroplasty. 2014;29(9):1774-8. 
276. Rader CP, Sterner T, Jakob F, Schütze N, Eulert J. Cytokine response of human macrophage-like cells after contact with polyethylene and pure titanium particles. J Arthroplasty. 1999;14(7):840-8.

277. Fuentes ME, Durham SK, Swerdel MR, Lewin AC, Barton DS, Megill JR, et al. Controlled recruitment of monocytes and macrophages to specific organs through transgenic expression of monocyte chemoattractant protein-1. J Immunol. 1995;155(12):5769-76.

278. Fritz EA, Glant TT, Vermes C, Jacobs JJ, Roebuck KA. Titanium particles induce the immediate early stress responsive chemokines IL- 8 and MCP-1 in osteoblasts. J Orthop Res. 2002;20(3):490-8.

279. Queally J, Devitt B, Butler J, Malizia A, Murray D, Doran P, et al. Cobalt ions induce chemokine secretion in primary human osteoblasts. J Orthop Res. 2009;27(7):855-64.

280. Caicedo MS, Pennekamp PH, McAllister K, Jacobs JJ, Hallab NJ. Soluble ions more than particulate cobalt-alloy implant debris induce monocyte costimulatory molecule expression and release of proinflammatory cytokines critical to metalinduced lymphocyte reactivity. J Biomed Mater Res A. 2010;93(4):1312-21.

281. Shanbhag AS, Kaufman AM, Hayata K, Rubash HE. Assessing osteolysis with use of high-throughput protein chips. J Bone Joint Surg. 2007;89(5):1081-9.

282. Wang JY, Wicklund BH, Gustilo RB, Tsukayama DT. Titanium, chromium and cobalt ions modulate the release of bone-associated cytokines by human monocytes/macrophages in vitro. Biomaterials. 1996;17(23):2233-40. 

IMPLANTS

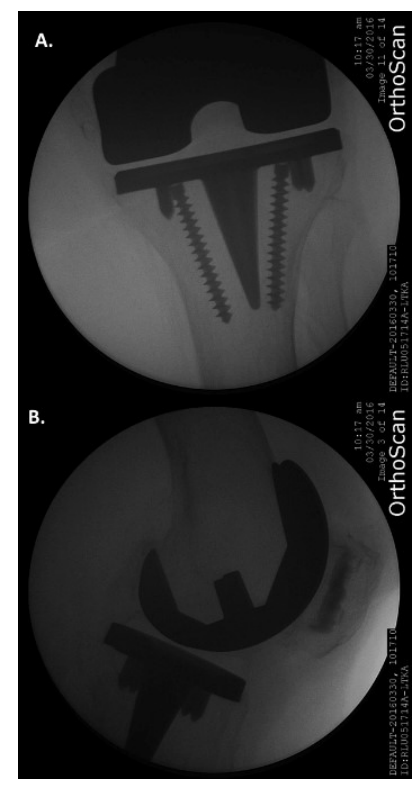

Figure A-1. Fluoroscopic A) AP and B) lateral views of specimen DRLU051714-L

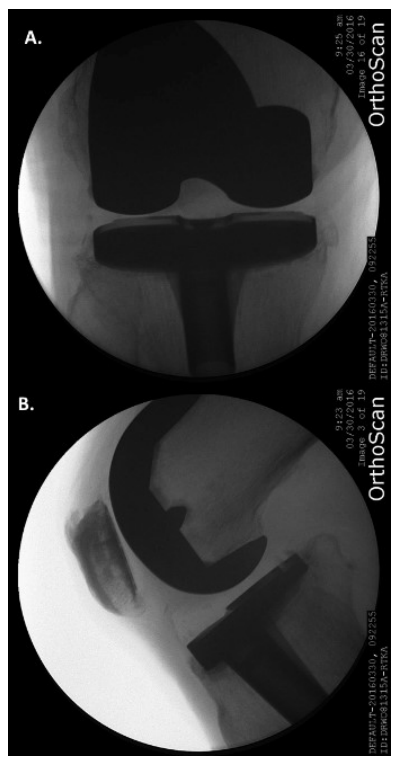

Figure A-2. Fluoroscopic A) AP and B) lateral views of specimen DRLU081315-R 


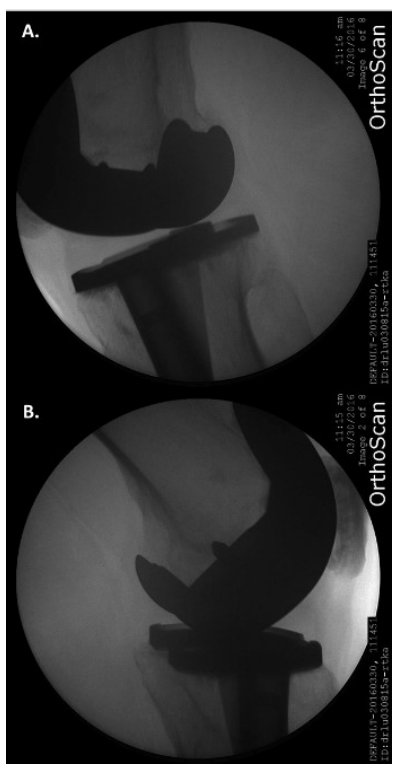

Figure A-3. A) Medial and B) lateral views of specimen DRLU081315-R on a fluoroscopy-guided radiograph

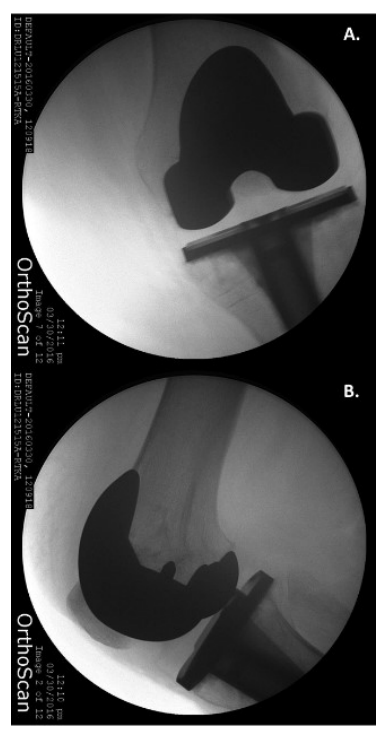

Figure A-4. Fluoroscopic A) AP and B) lateral views of specimen DRLU121515-R 


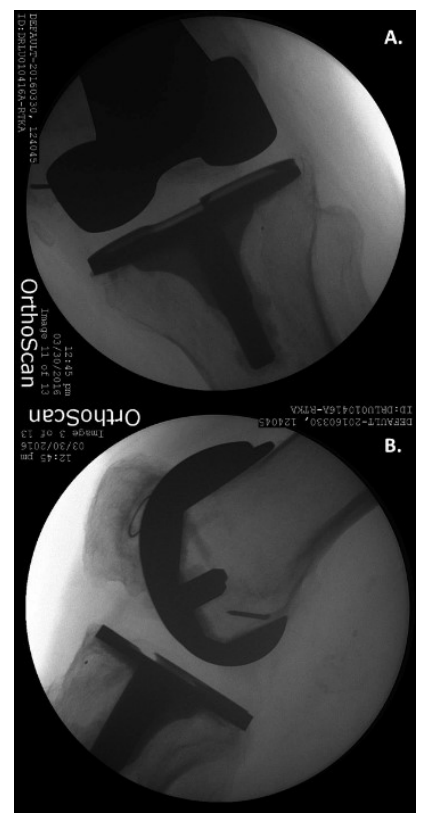

Figure A-5. Fluoroscopic A) AP and B) lateral views of specimen DRLU010416-R

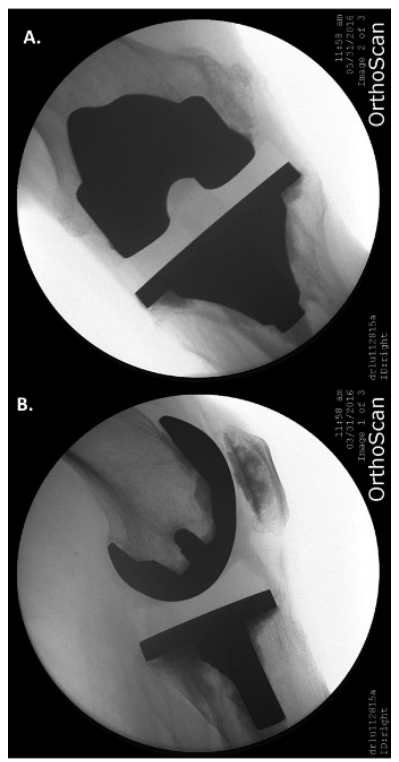

Figure A-6. Fluoroscopic A) AP and B) lateral views of specimen DRLU112815-R 


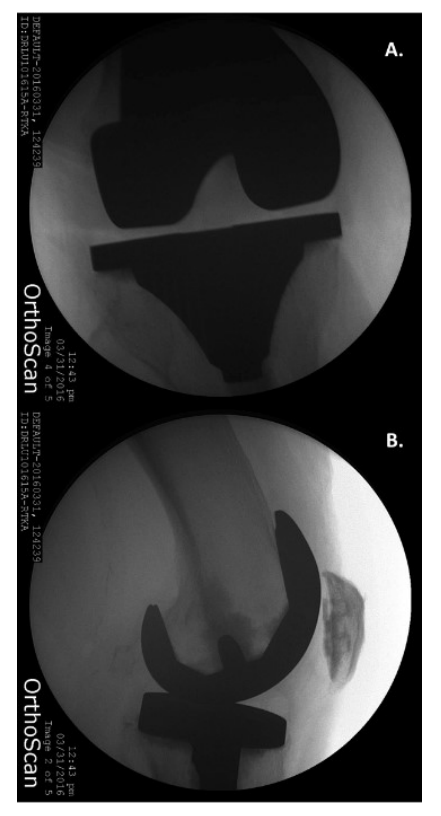

Figure A-7. Fluoroscopic A) AP and B) lateral views of specimen DRLU101615-R

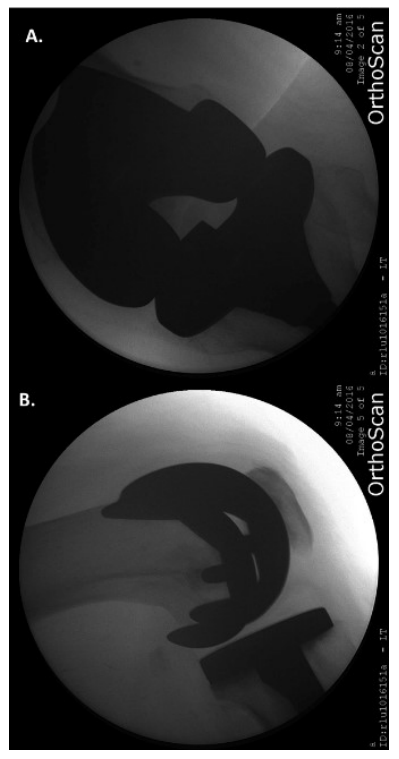

Figure A-8. Fluoroscopic views of specimen RLU1016151A-L 


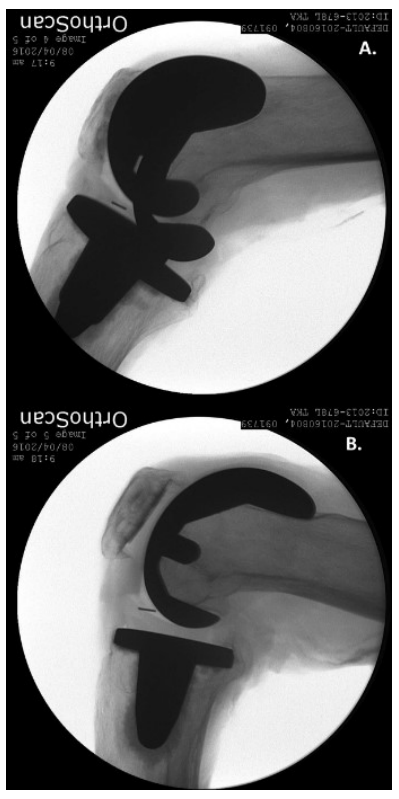

Figure A-9. Fluoroscopic A) oblique posterior condylar and B) lateral views of specimen 2013-678L

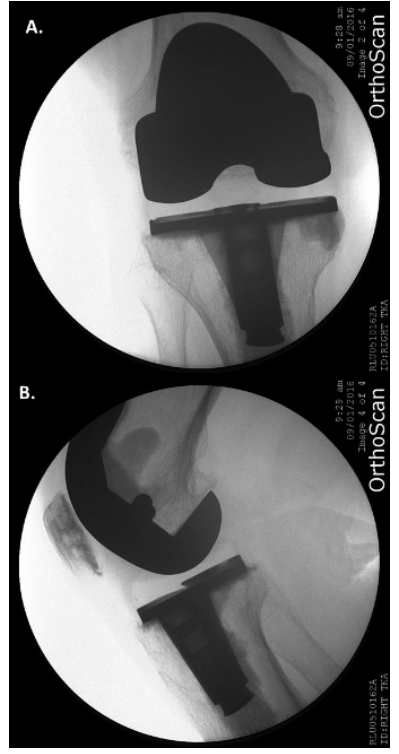

Figure A-10. Fluoroscopic A) AP and B) lateral views of specimen RLU0510162A$\mathbf{R}$ 


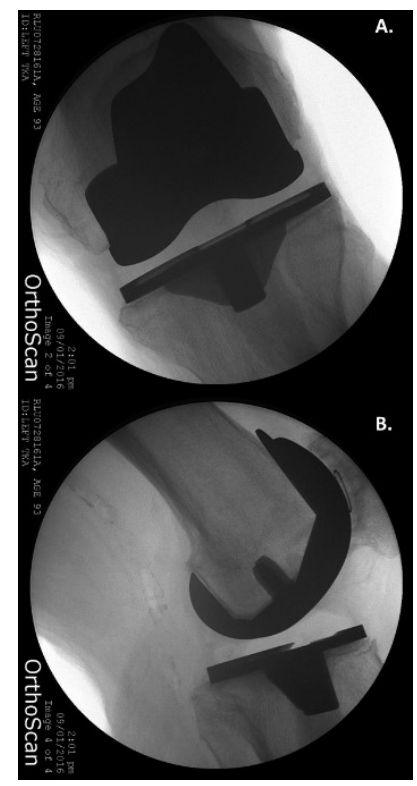

Figure A-11. Fluoroscopic A) AP and B) lateral views of specimen RLU0728161A-L

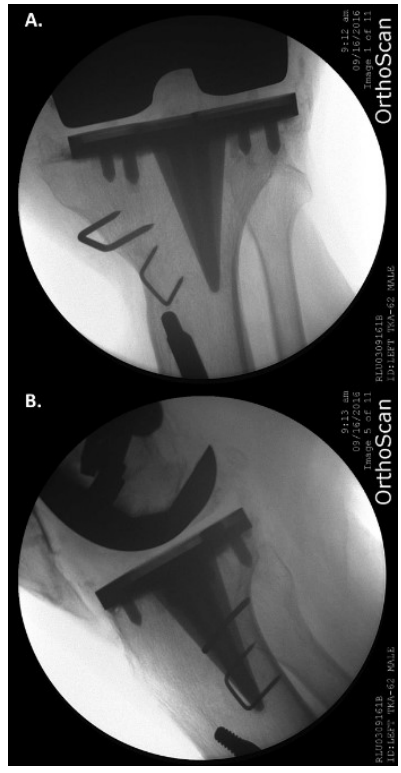

Figure A-12. Fluoroscopic A) AP and B) lateral views of specimen RLU0309161B-L 


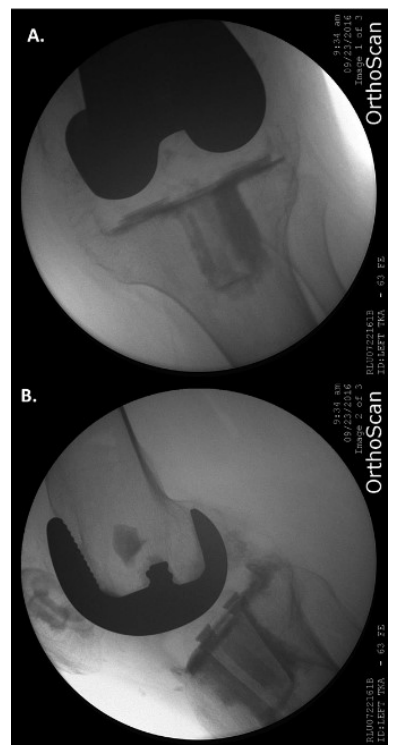

Figure A-13. Fluoroscopic A) AP and B) lateral views of specimen RLU0722161B-L

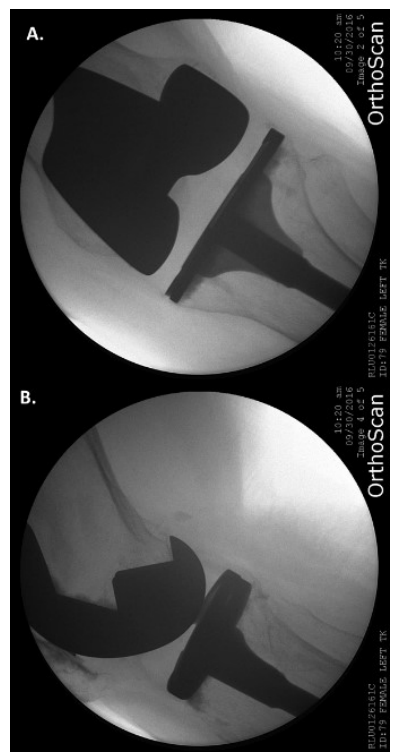

Figure A-14. Fluoroscopic A) AP and B) lateral views of specimen RLU0126161C-L 


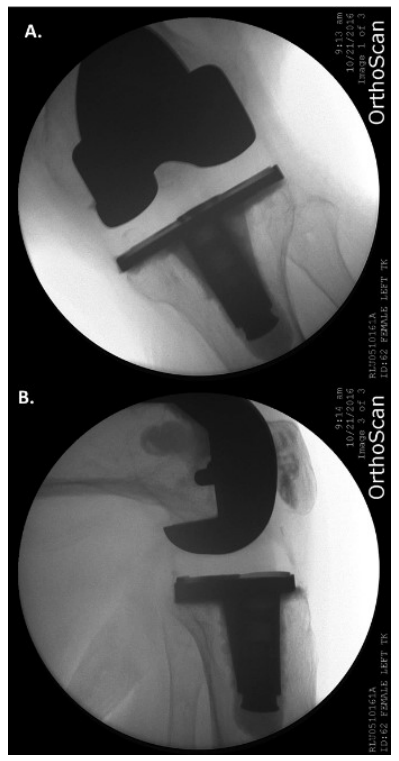

Figure A-15. Fluoroscopic A) AP and B) lateral views of specimen RLU0510161A-L

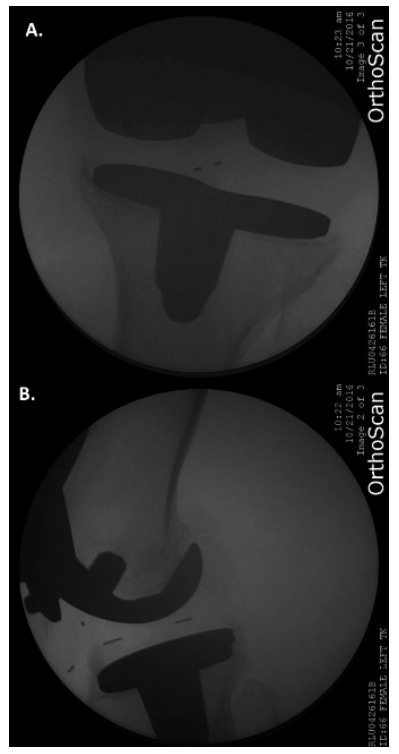

Figure A-16. Fluoroscopic A) AP and B) lateral views of specimen RLU0426161B-L 


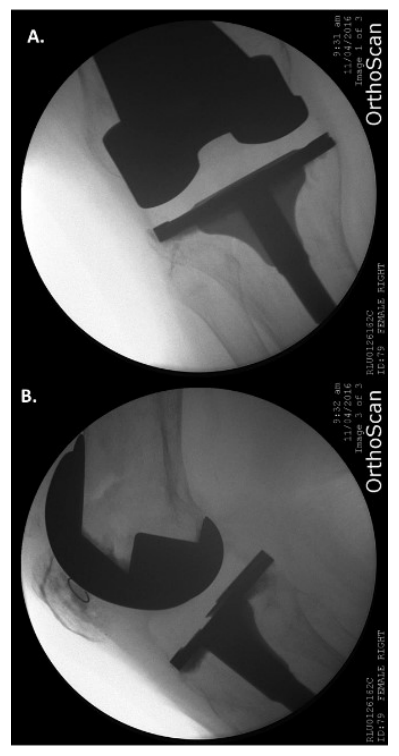

Figure A-17. Fluoroscopic A) AP and B) lateral views of specimen RLU0126162C$\mathbf{R}$

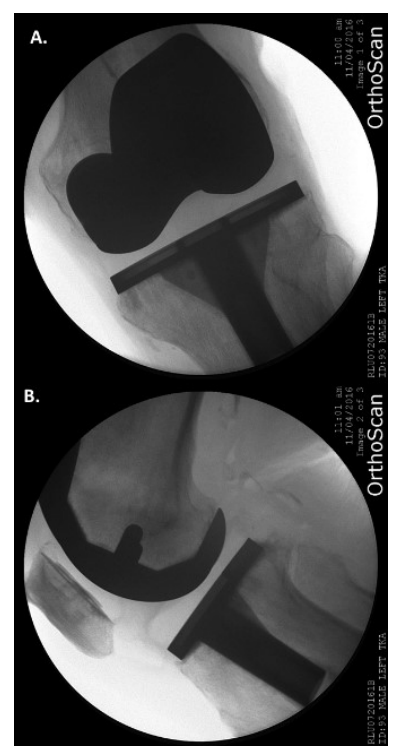

Figure A-18. Fluoroscopic A) AP and B) lateral views of specimen RLU0720161B-L 


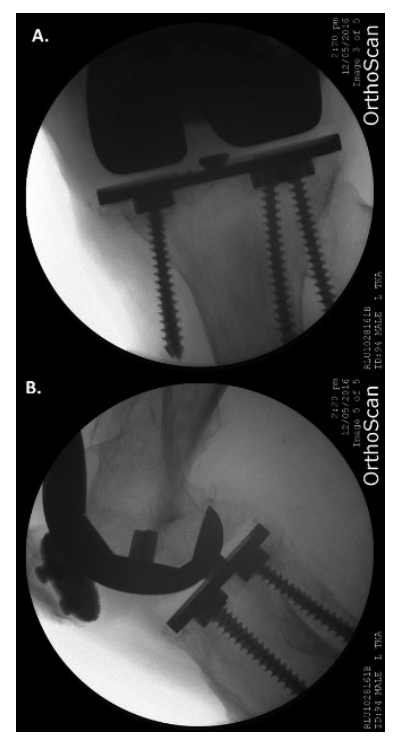

Figure A-19. Fluoroscopic A) AP and B) lateral views of specimen RLU1028161B-L

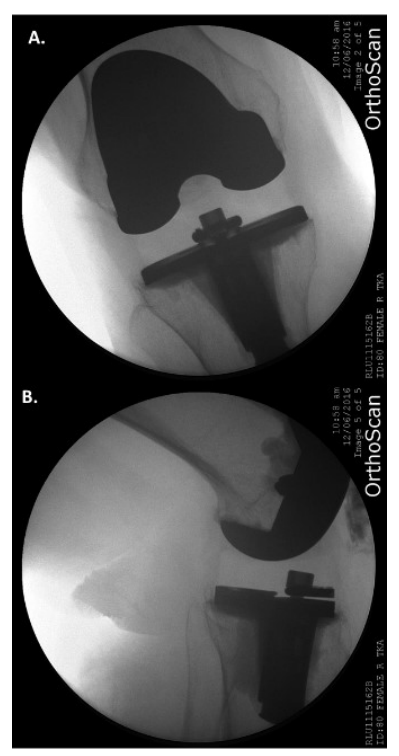

Figure A-20. Fluoroscopic A) AP and B) lateral views of specimen RLU1115162B-R 


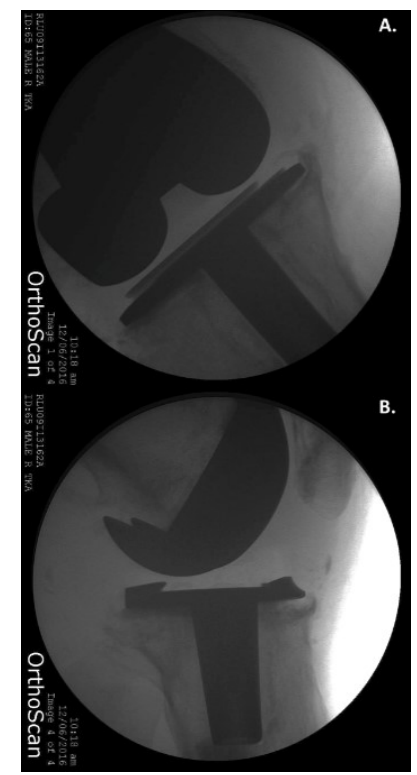

Figure A-21. Fluoroscopic A) AP and B) lateral views of specimen RLU0913162A$\mathbf{R}$

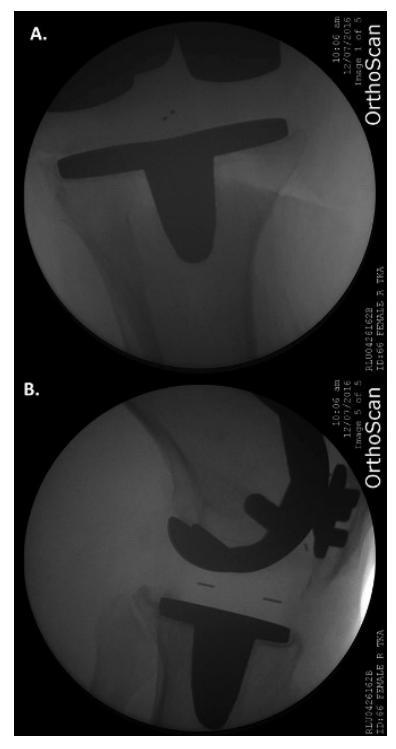

Figure A-22. Fluoroscopic A) AP and B) lateral views of specimen RLU0426162B-R 


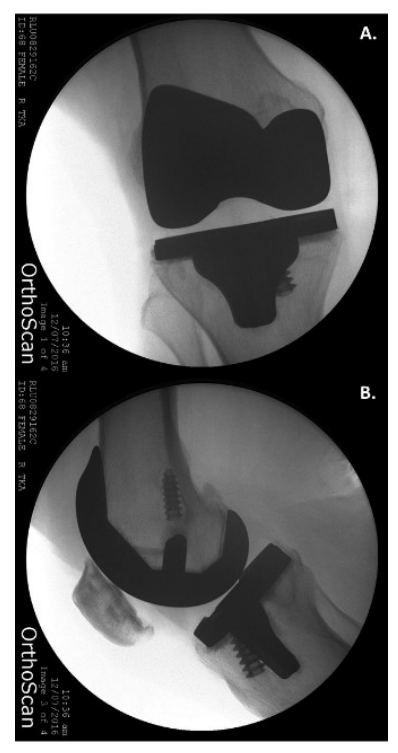

Figure A-23. Fluoroscopic A) AP and B) lateral views of specimen RLU0829162CR

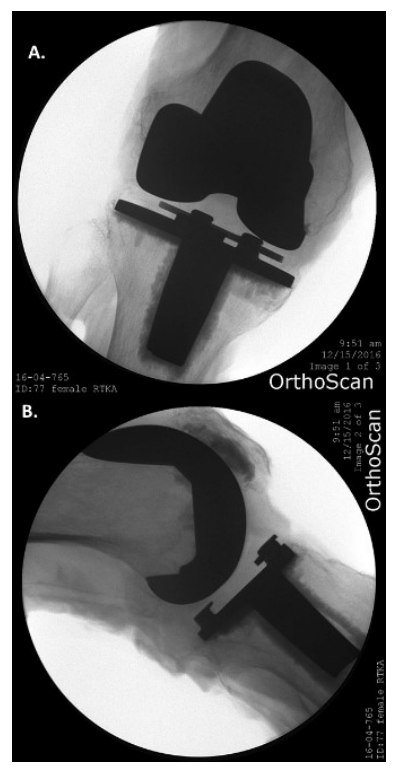

Figure A-24. Fluoroscopic A) AP and B) lateral views of specimen 16-04-765R 


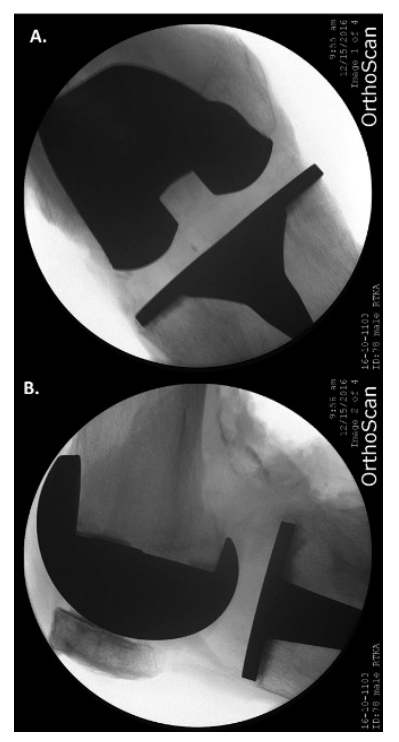

Figure A-25. Fluoroscopic A) AP and B) lateral views of specimen 16-10-1103R

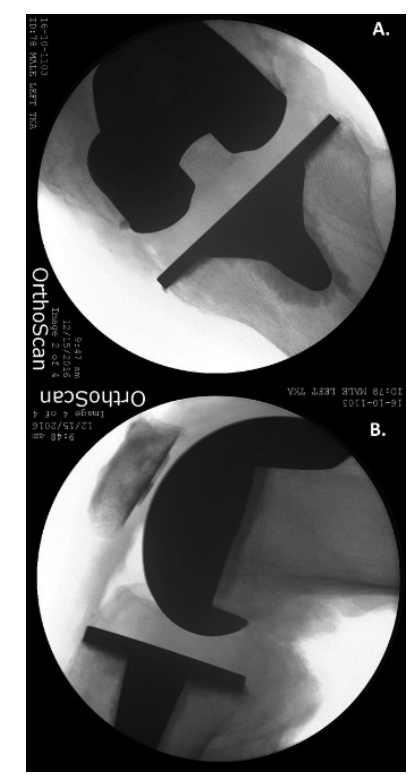

Figure A-26. Fluoroscopic A) AP and B) lateral views of specimen 16-10-1103L 


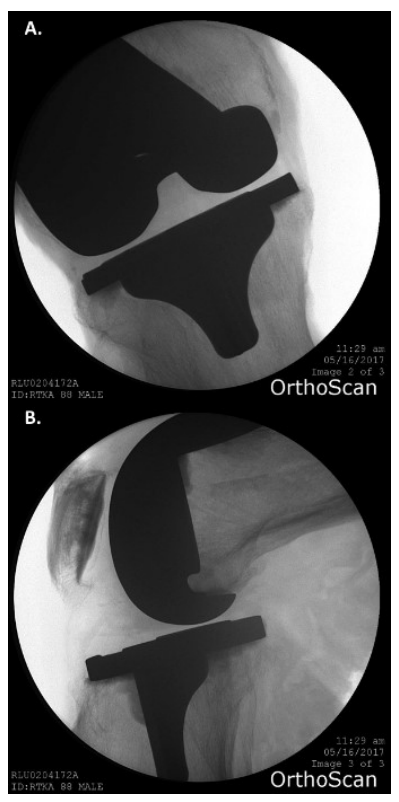

Figure A-27. Fluoroscopic A) AP and B) lateral views of specimen RLU0204172AR

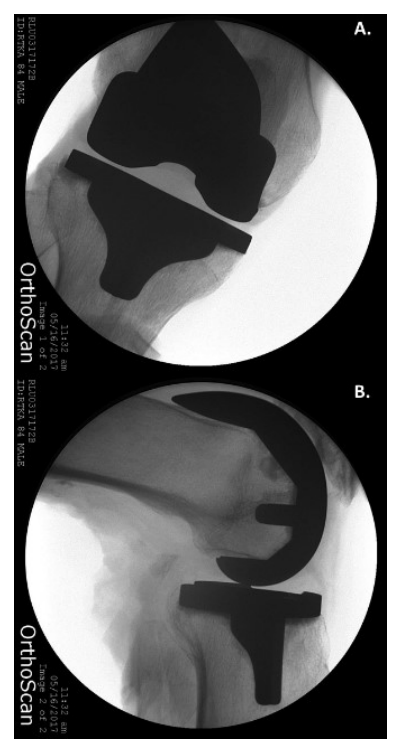

Figure A-28. Fluoroscopic A) AP and B) lateral views of specimen RLU0317172B-R 


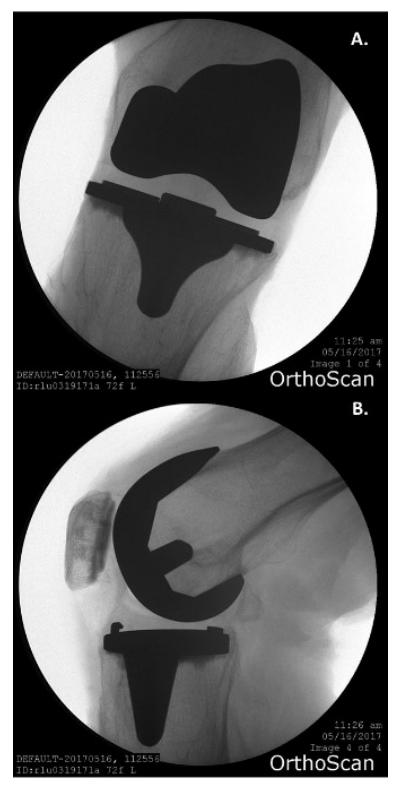

Figure A-29. Fluoroscopic A) AP and B) lateral views of specimen RLU0319171A-L

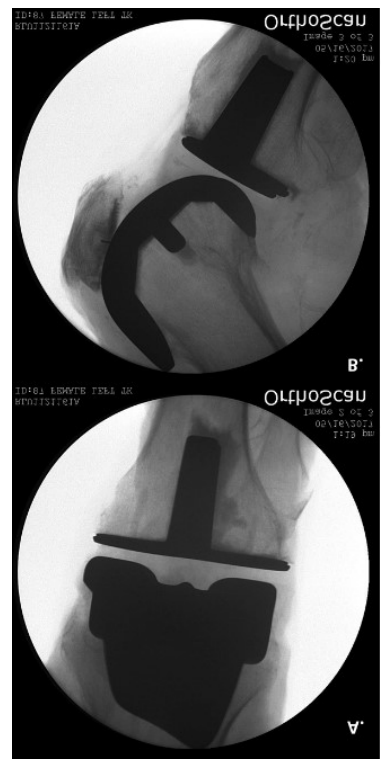

Figure A-30. Fluoroscopic A) AP and B) lateral views of specimen RLU1121161A-L 


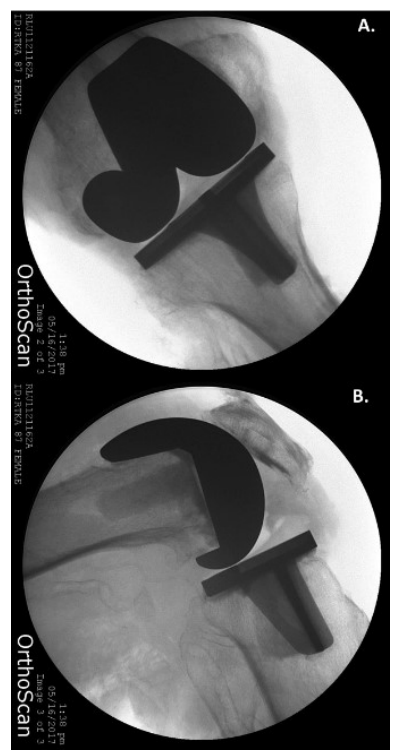

Figure A-31. Fluoroscopic A) AP and B) lateral views of specimen RLU1121162A$\mathbf{R}$

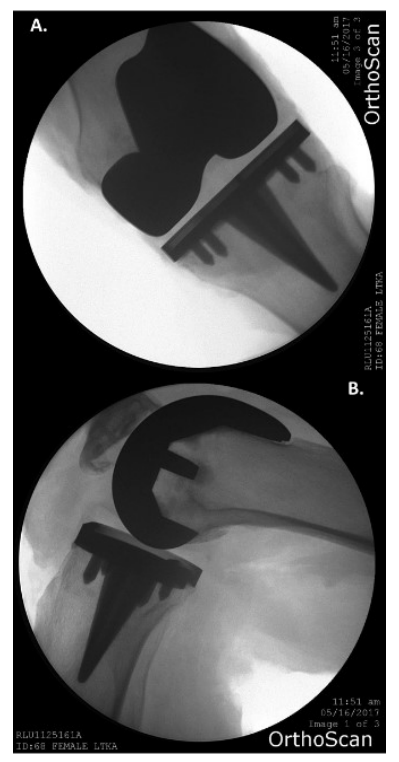

Figure A-32. Fluoroscopic A) AP and B) lateral views of specimen RLU1125161A-L 


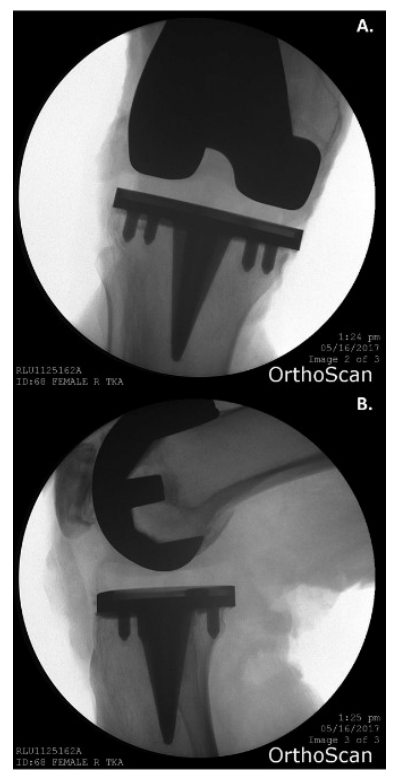

Figure A-33. Fluoroscopic A) AP and B) lateral views of specimen RLU1125162AR 


\section{APPENDIX B. CORRELATIONS BETWEEN LAXITY AND TISSUE METAL CONCENTRATIONS}

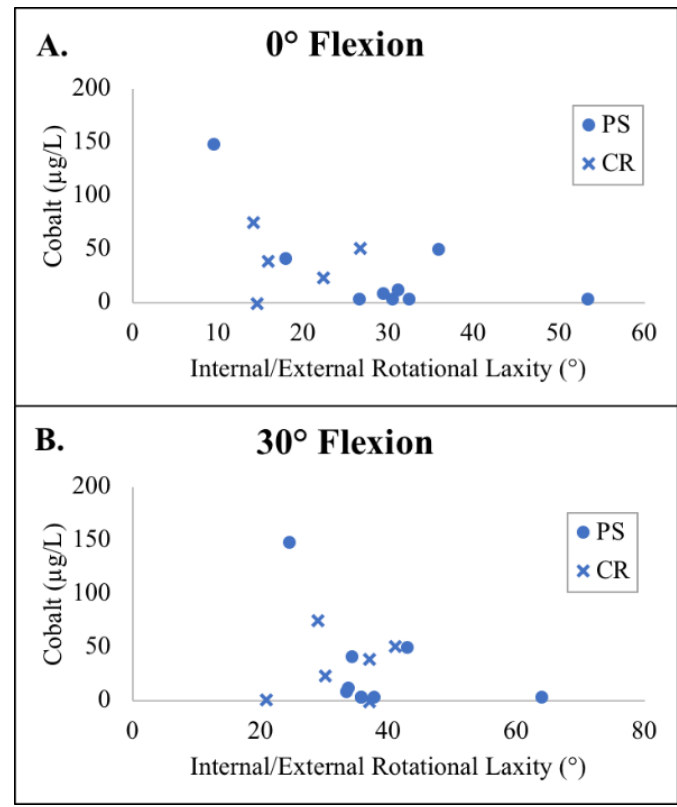

Figure B-1. Relationship between IE rotational laxity at (A) $0^{\circ}$ and (B) $30^{\circ}$ of flexion and Co concentrations in periprosthetic tissue 


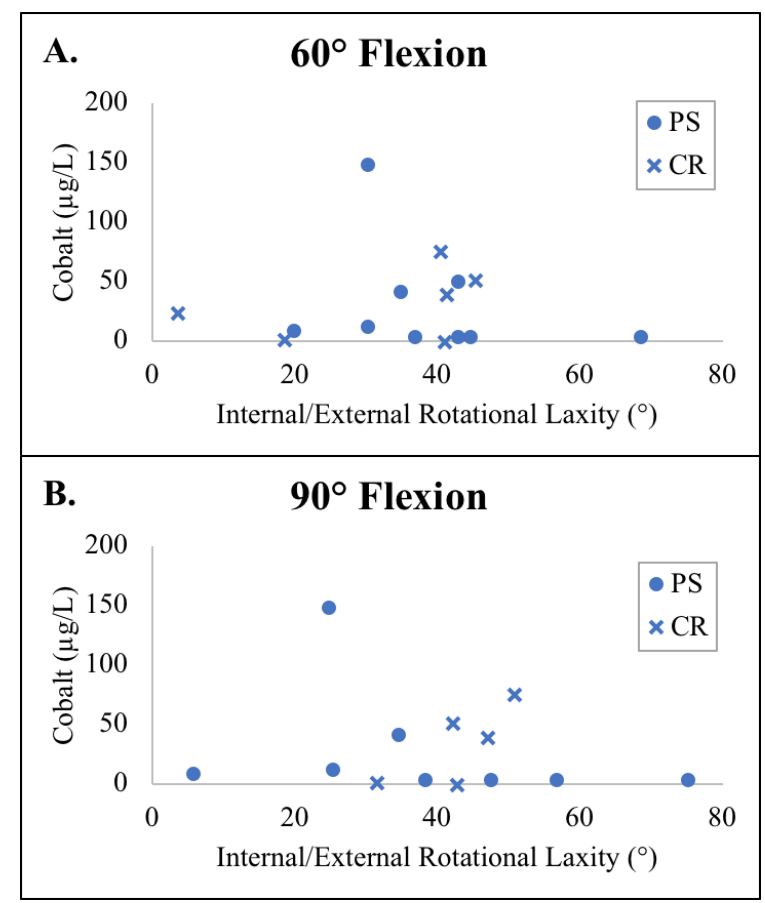

Figure B-2. Relationship between IE rotational laxity at (A) $60^{\circ}$ and (B) $90^{\circ}$ of flexion and Co concentrations in periprosthetic tissue

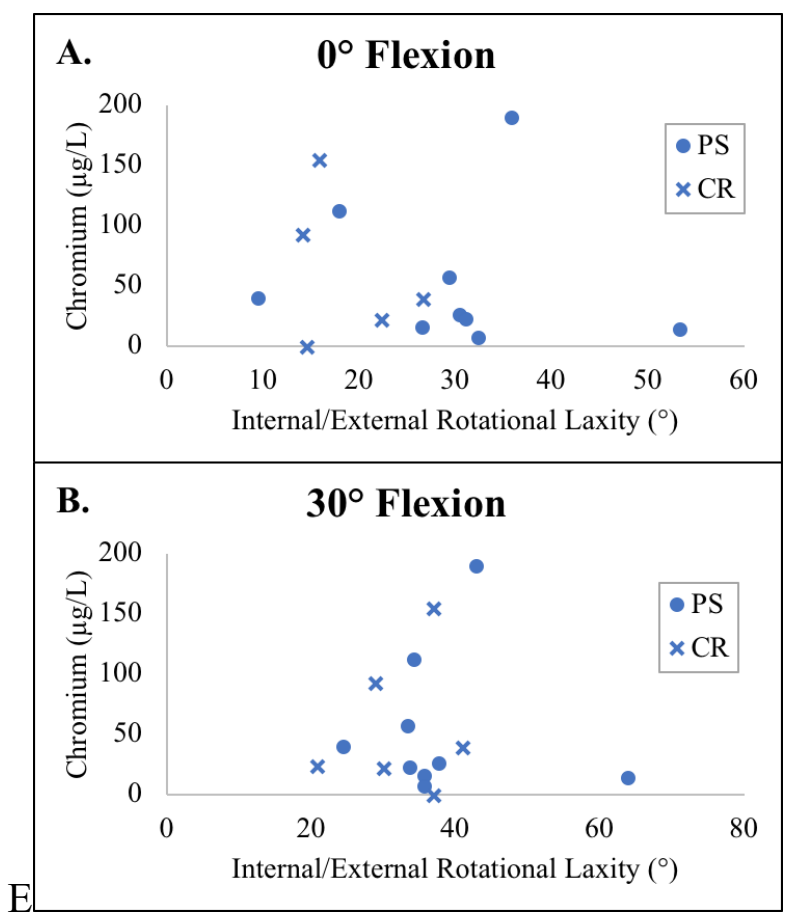

Figure B-3. Relationship between IE rotational laxity at (A) $0^{\circ}$ and (B) $30^{\circ}$ of flexion and $\mathrm{Cr}$ concentrations in periprosthetic tissue 


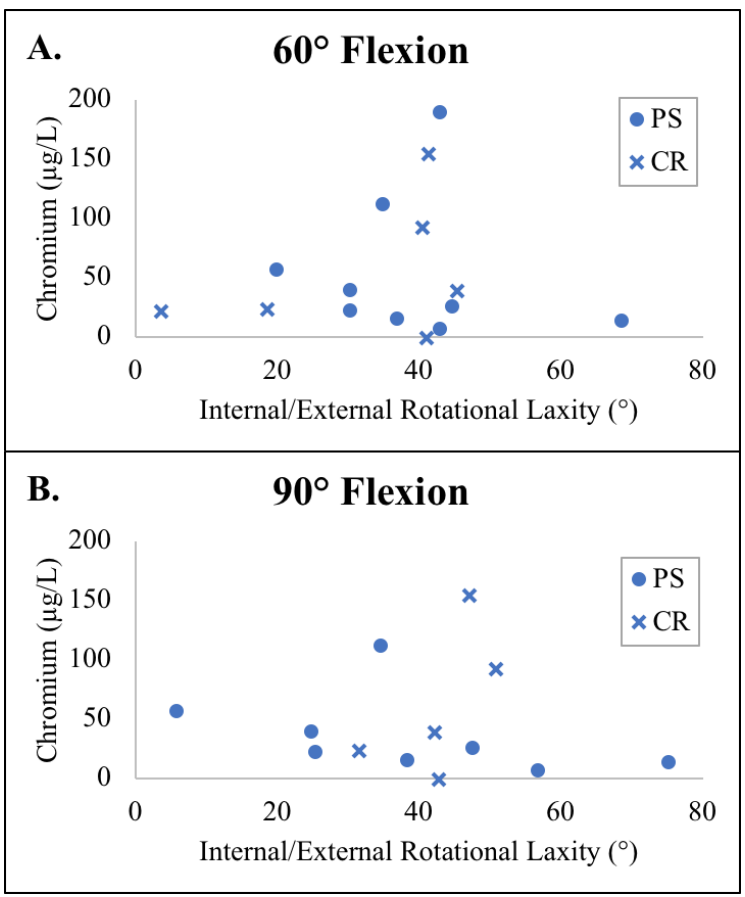

Figure B-4. Relationship between IE rotational laxity at (A) $60^{\circ}$ and (B) $90^{\circ}$ of flexion and $\mathrm{Cr}$ concentrations in periprosthetic tissue

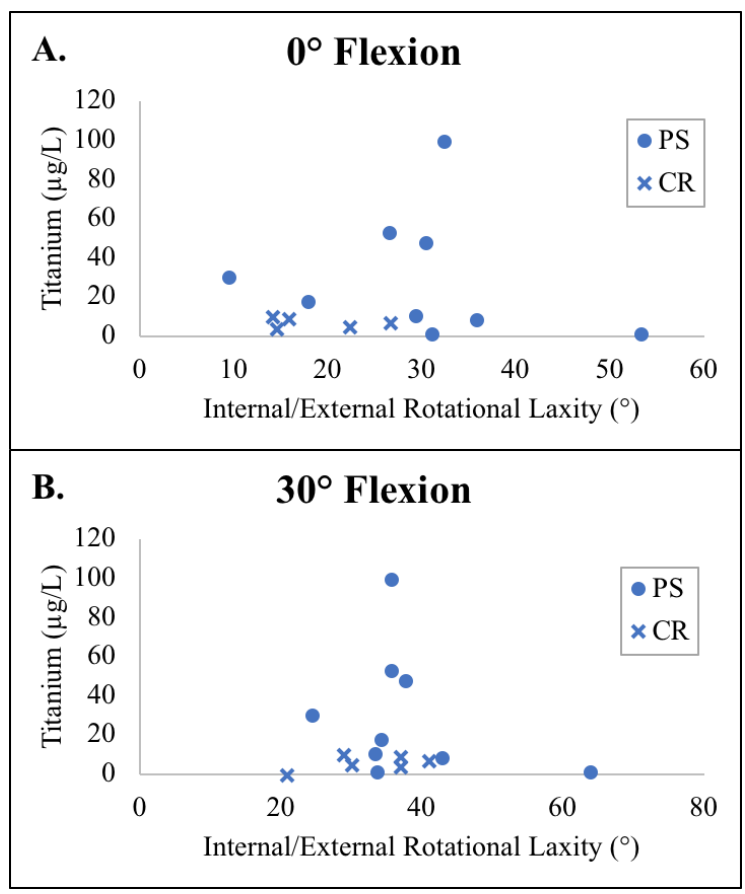

Figure B-5. Relationship between IE rotational laxity at (A) $0^{\circ}$ and (B) $30^{\circ}$ of flexion and $\mathrm{Ti}$ concentrations in periprosthetic tissue 


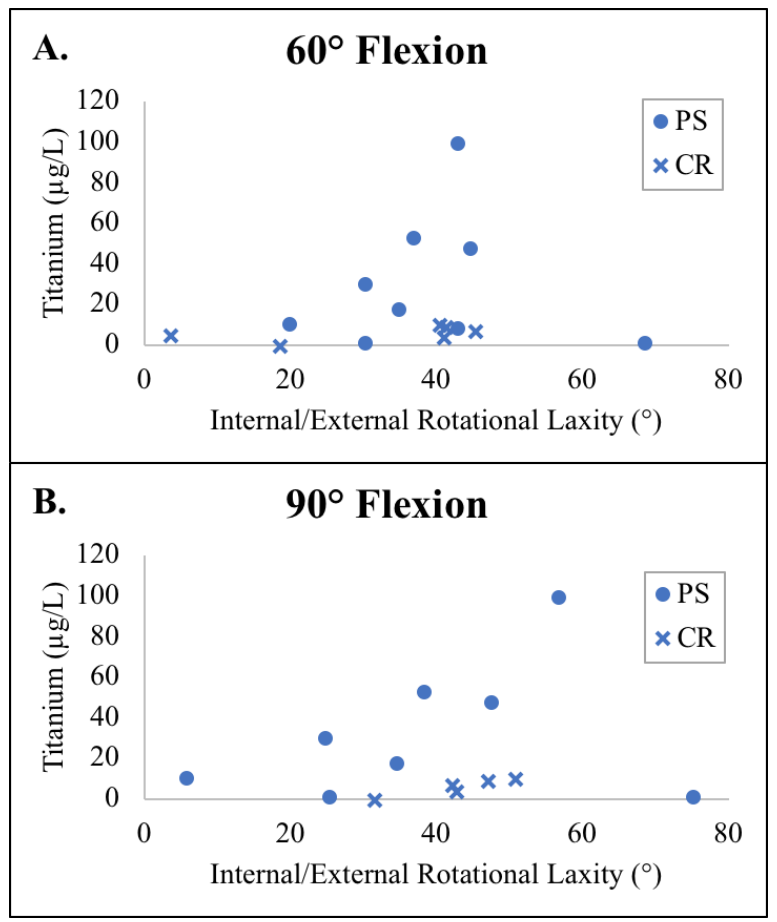

Figure B-6. Relationship between IE rotational laxity at (A) $60^{\circ}$ and (B) $90^{\circ}$ of flexion and $T i$ concentration in periprosthetic tissue

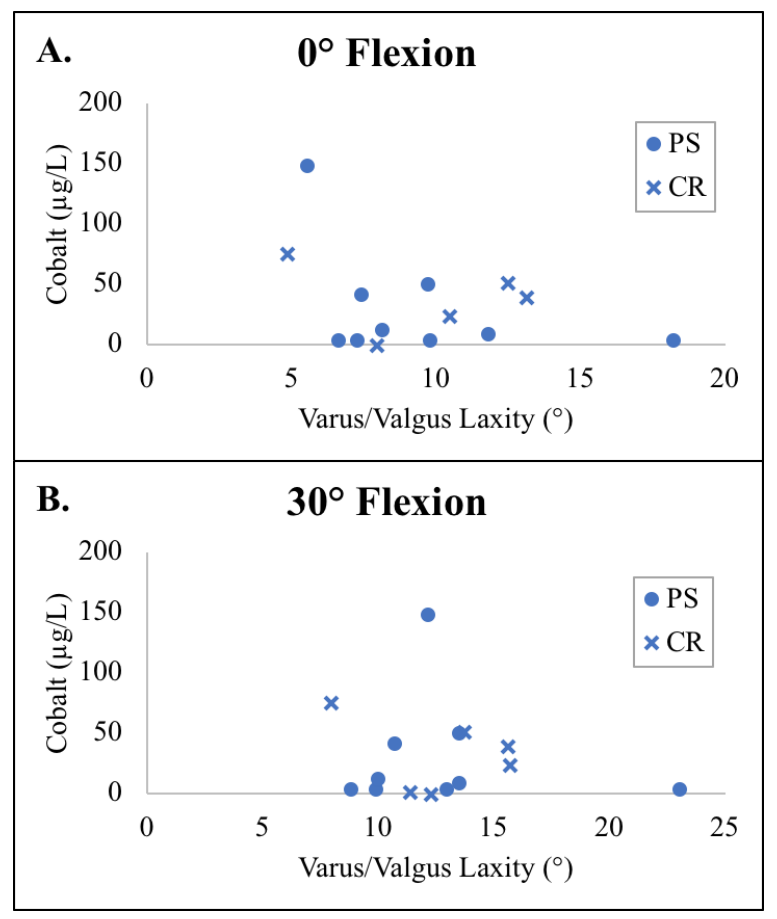

Figure B-7. Relationship between VV laxity at (A) $0^{\circ}$ and (B) $30^{\circ}$ of flexion and Co concentrations in periprosthetic tissue 


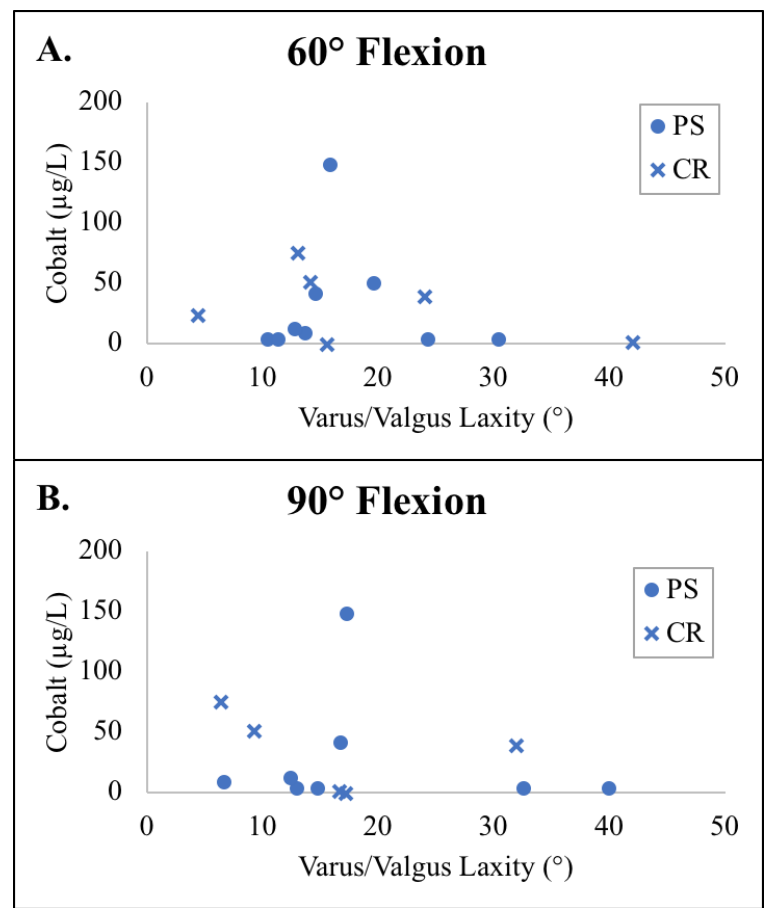

Figure B-8. Relationship between VV laxity at (A) $60^{\circ}$ and (B) $90^{\circ}$ of flexion and Co concentrations in periprosthetic tissue

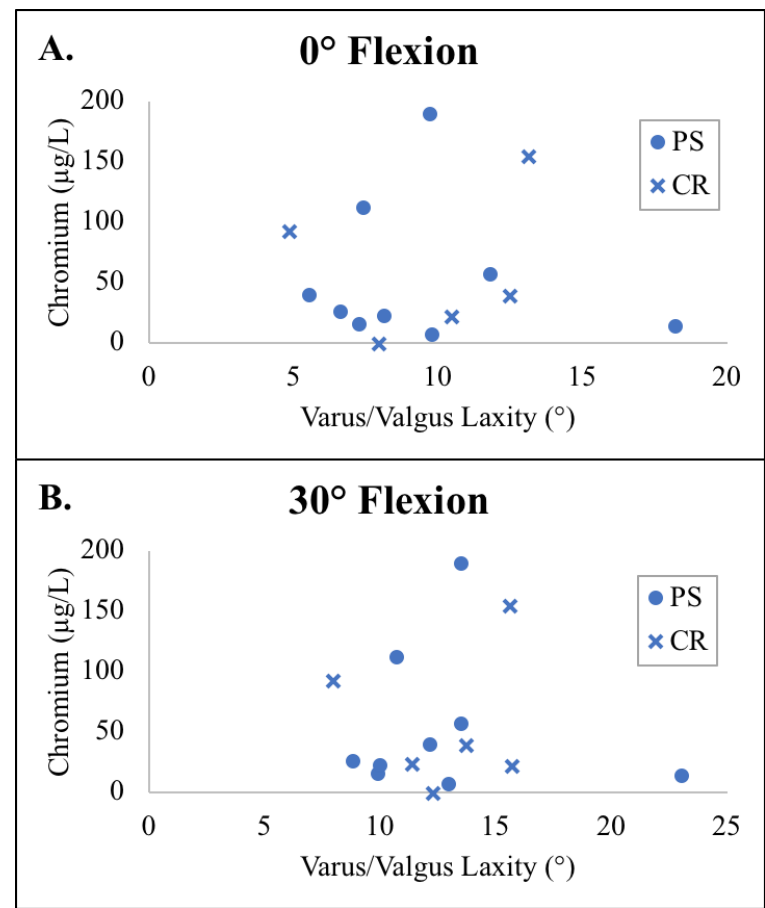

Figure B-9. Relationship between VV laxity at (A) $0^{\circ}$ and (B) $30^{\circ}$ of flexion and $\mathrm{Cr}$ concentrations in periprosthetic tissue 


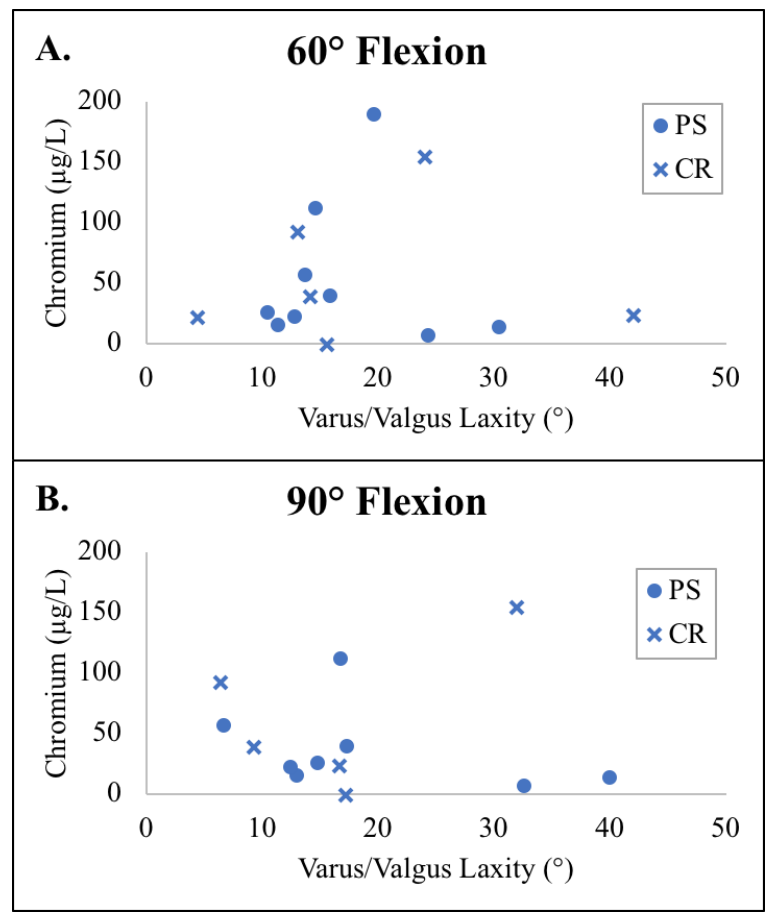

Figure B-10. Relationship between VV laxity at (A) $60^{\circ}$ and (B) $90^{\circ}$ of flexion and Cr concentrations in periprosthetic tissue

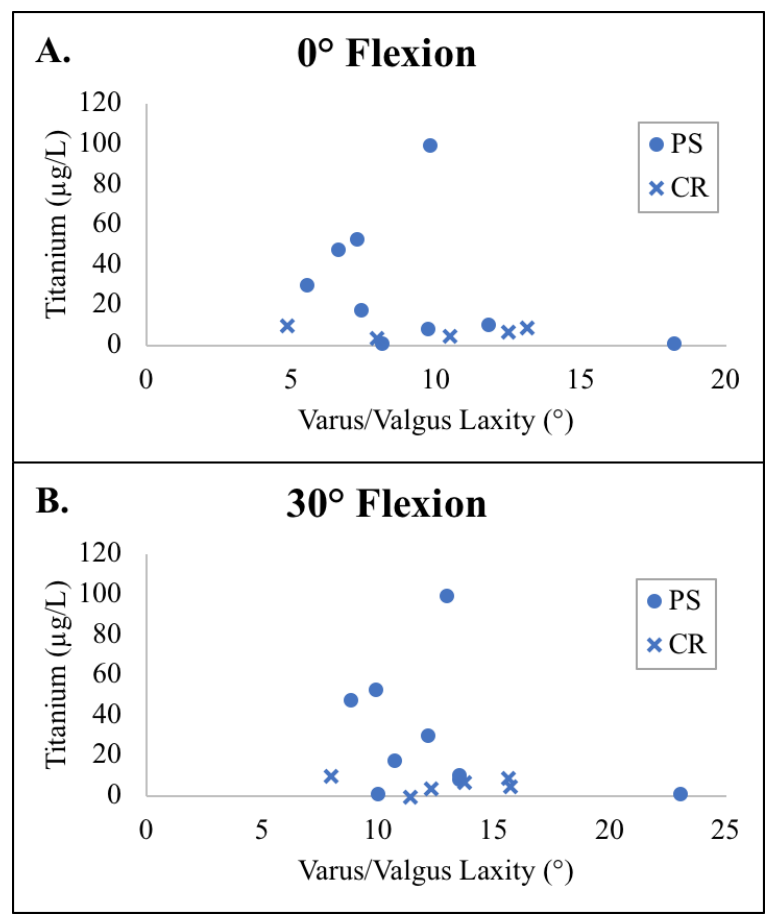

Figure B-11. Relationship between VV laxity at (A) $0^{\circ}$ and (B) $30^{\circ}$ of flexion and Ti concentrations in periprosthetic tissue 


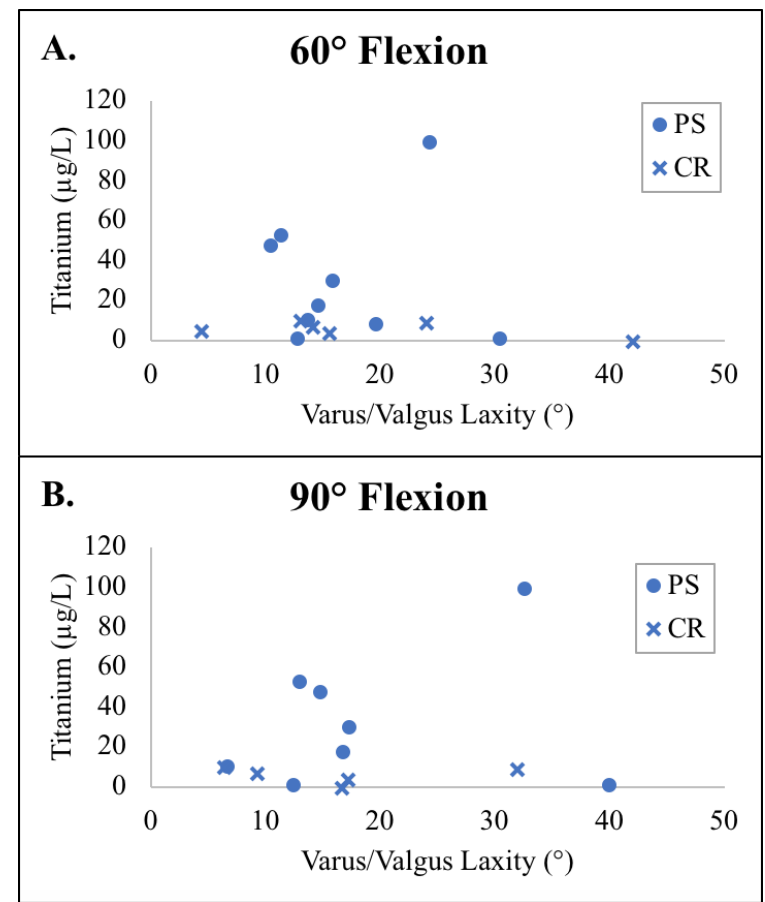

Figure B-12. Relationship between VV laxity at (A) $60^{\circ}$ and (B) $90^{\circ}$ of flexion and Ti concentrations in periprosthetic tissue

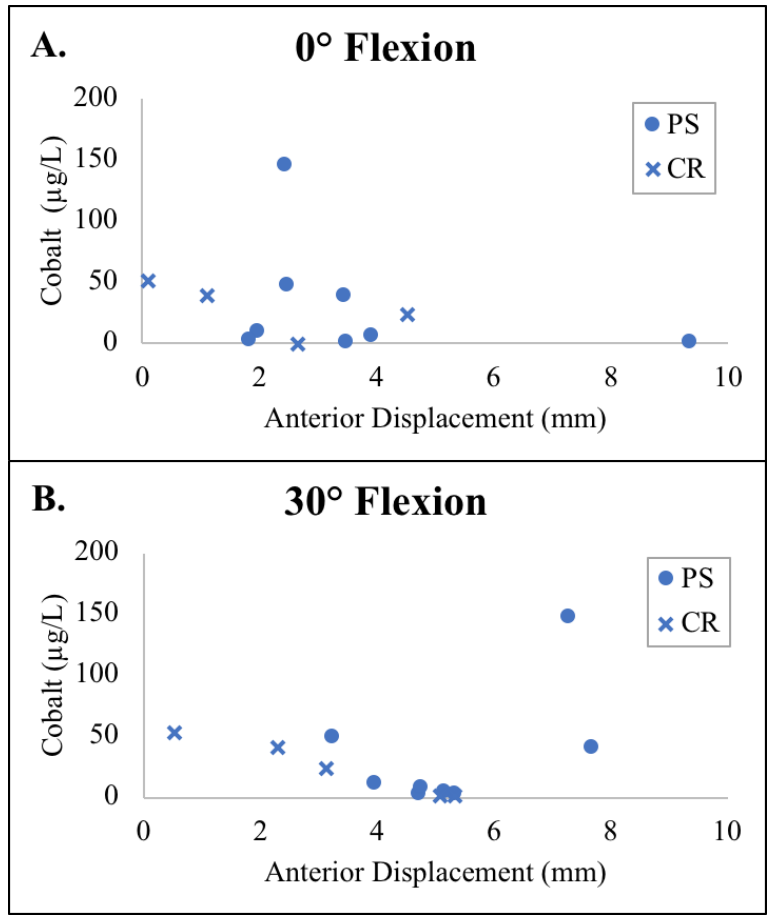

Figure B-13. Relationship between anterior displacement at (A) $0^{\circ}$ and (B) $30^{\circ}$ of flexion and Co concentrations in periprosthetic tissue 


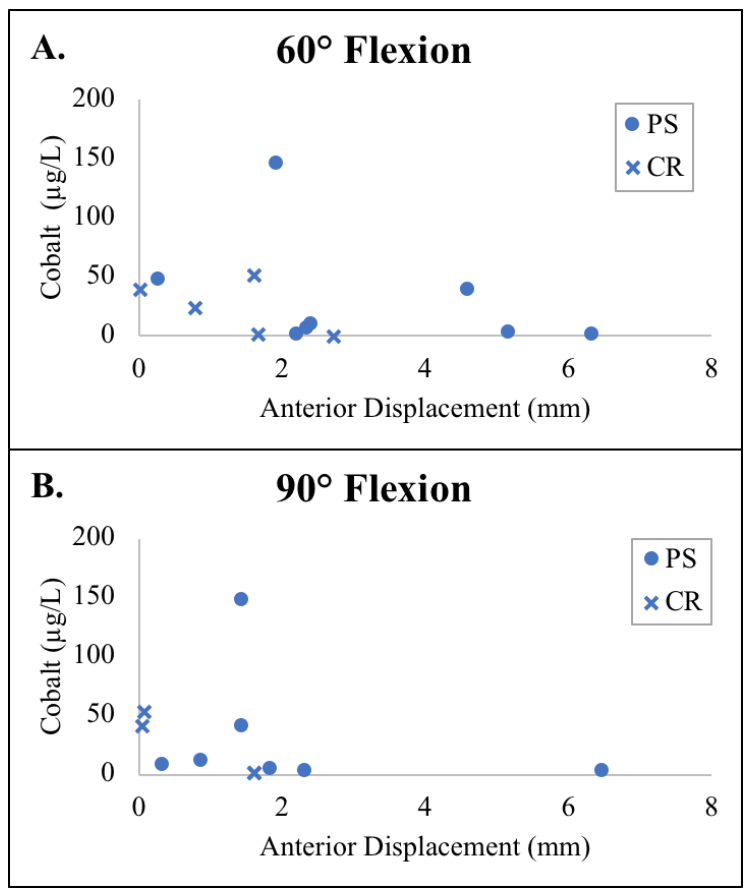

Figure B-14. Relationship between anterior displacement at (A) $60^{\circ}$ and (B) $90^{\circ}$ of flexion and Co concentrations in periprosthetic tissue

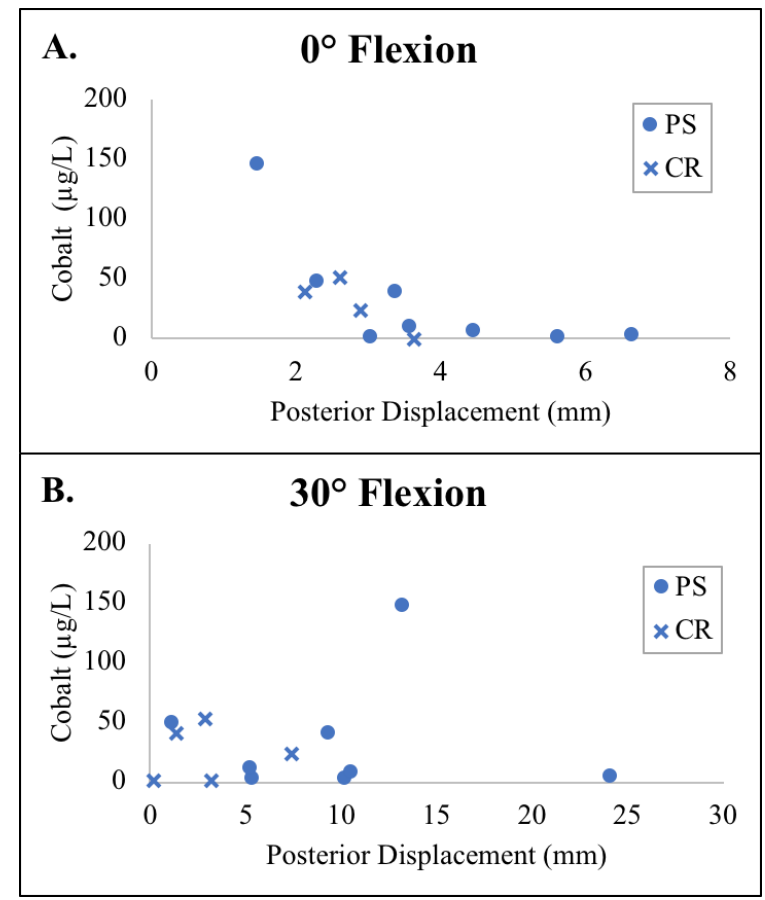

Figure B-15. Relationship between posterior displacement at (A) $0^{\circ}$ and (B) $30^{\circ}$ of flexion and Co concentrations in periprosthetic tissue 


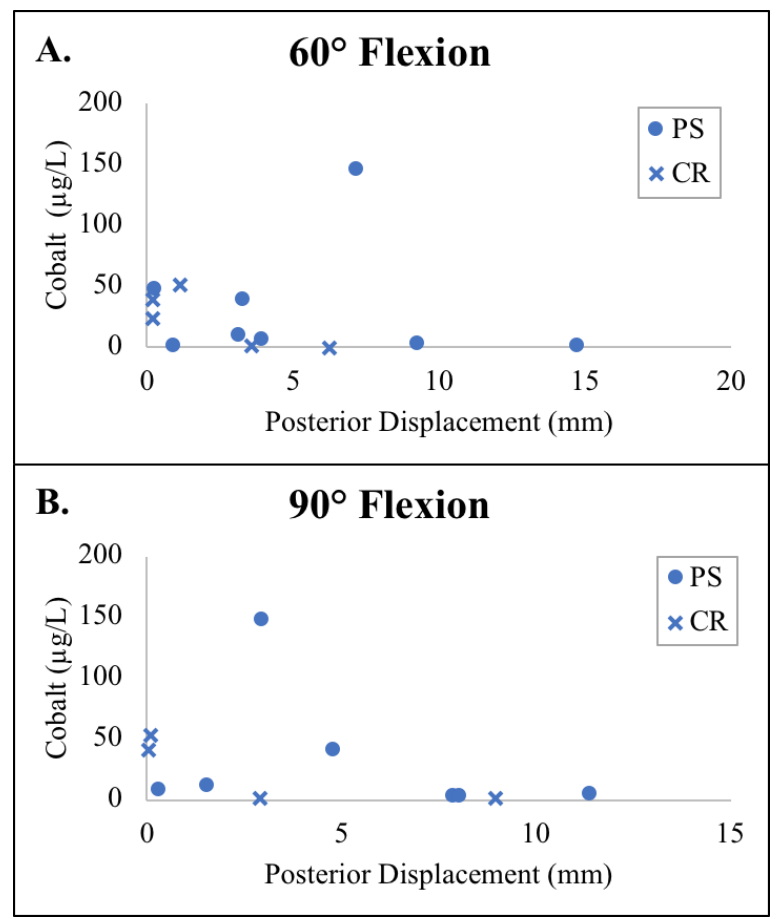

Figure B-16. Relationship between posterior displacement at (A) $60^{\circ}$ and (B) $90^{\circ}$ of flexion and Co concentrations in periprosthetic tissue

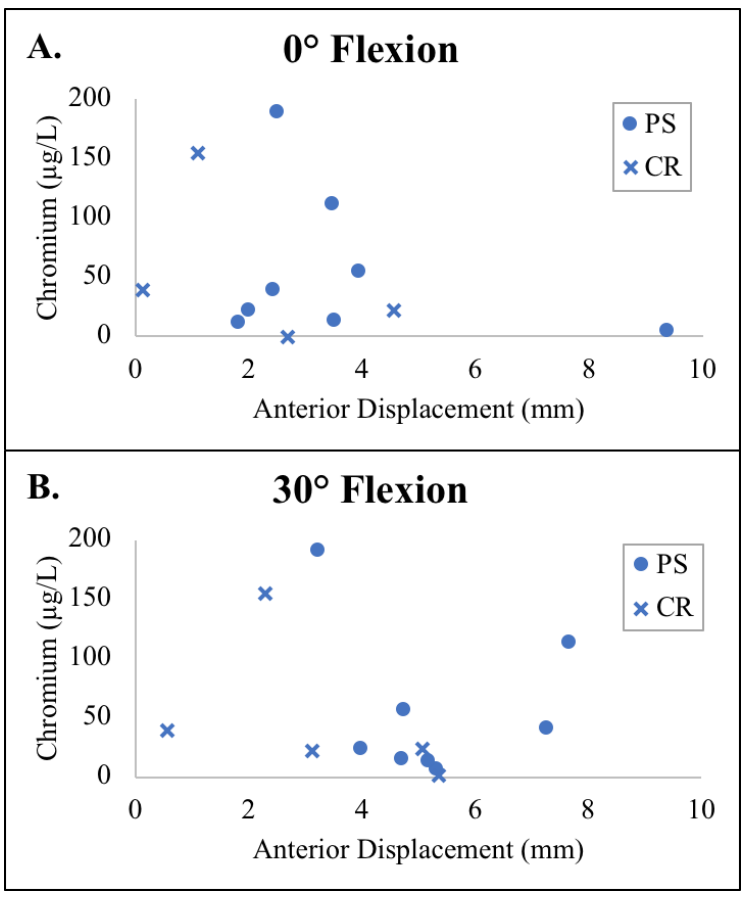

Figure B-17. Relationship between anterior displacement at (A) $0^{\circ}$ and (B) $30^{\circ}$ of flexion and $\mathrm{Cr}$ concentrations in periprosthetic tissue 


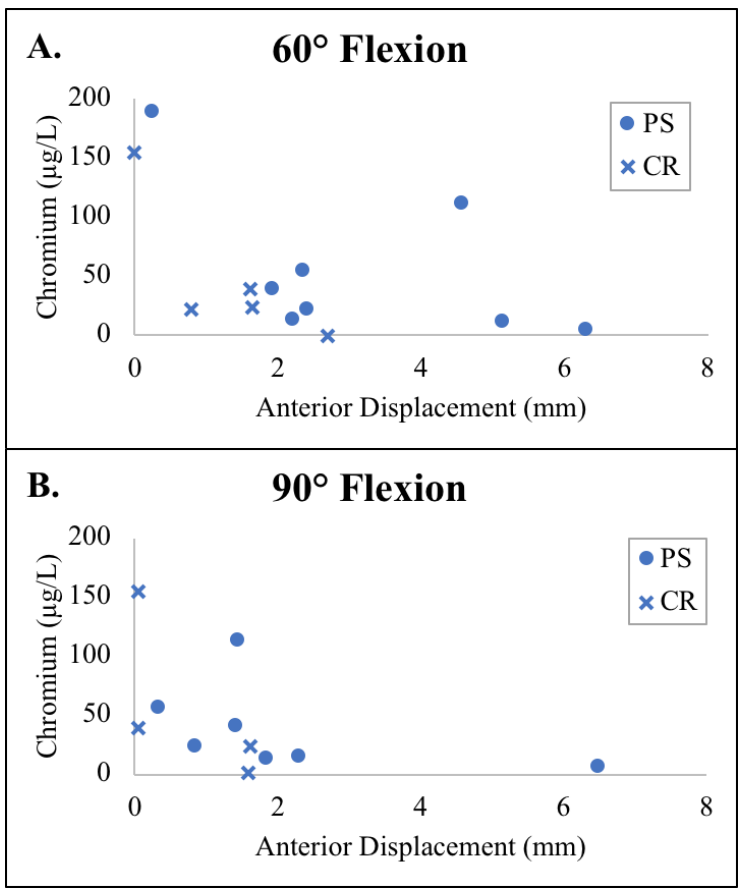

Figure B-18. Relationship between anterior displacement at (A) $60^{\circ}$ and (B) $90^{\circ}$ of flexion and $\mathrm{Cr}$ concentrations in periprosthetic tissue

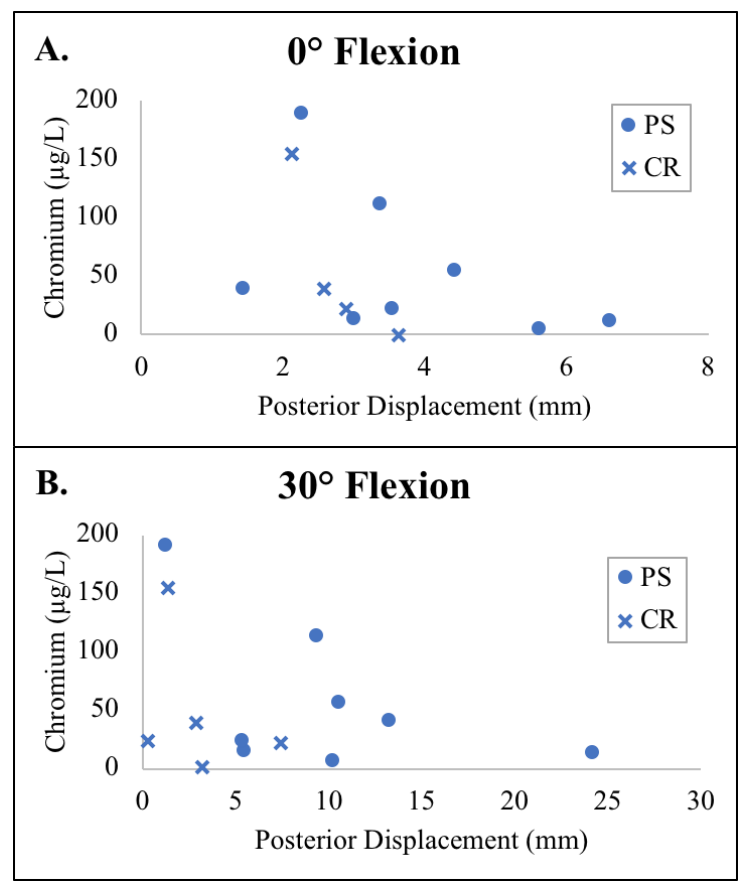

Figure B-19. Relationship between posterior displacement at (A) $0^{\circ}$ and (B) $30^{\circ}$ of flexion and $\mathrm{Cr}$ concentrations in periprosthetic tissue 


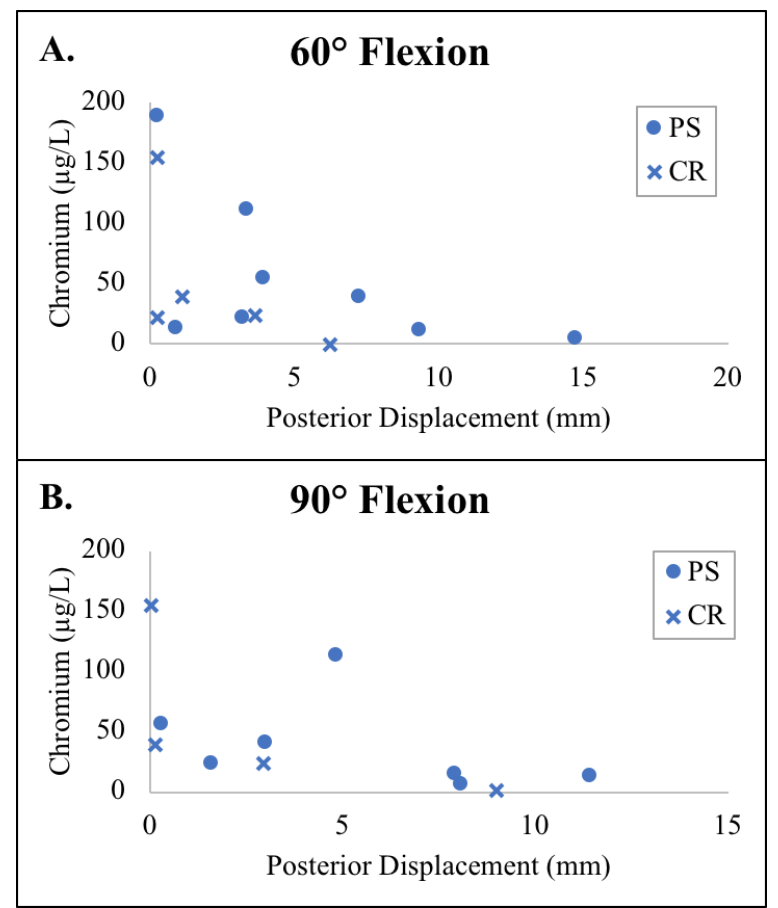

Figure B-20. Relationship between posterior displacement at (A) $60^{\circ}$ and (B) $90^{\circ}$ of flexion and $\mathrm{Cr}$ concentrations in periprosthetic tissue

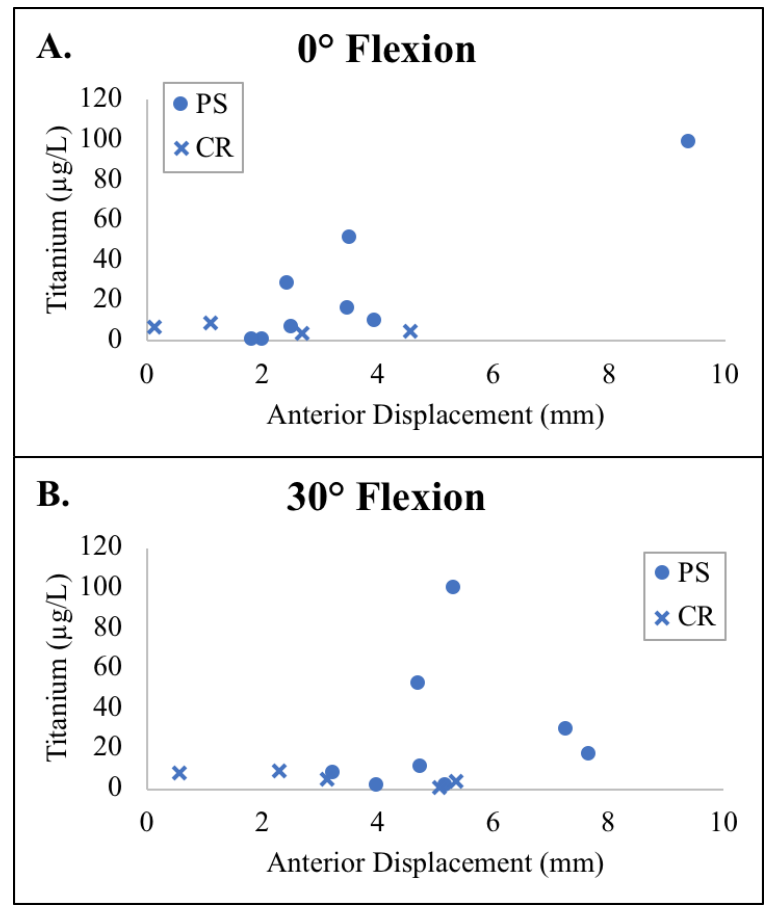

Figure B-21. Relationship between anterior displacement at (A) $0^{\circ}$ and (B) $30^{\circ}$ of flexion and $\mathrm{Ti}$ concentrations in periprosthetic tissue 


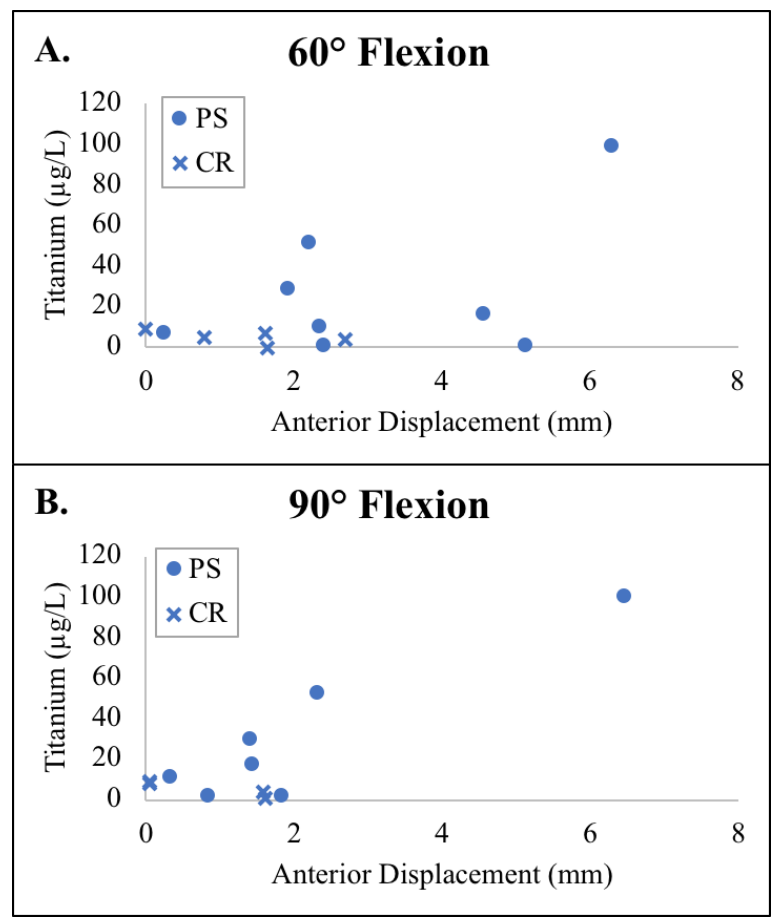

Figure B-22. Relationship between anterior displacement at (A) $60^{\circ}$ and (B) $90^{\circ}$ of flexion and $\mathrm{Ti}$ concentrations in periprosthetic tissue

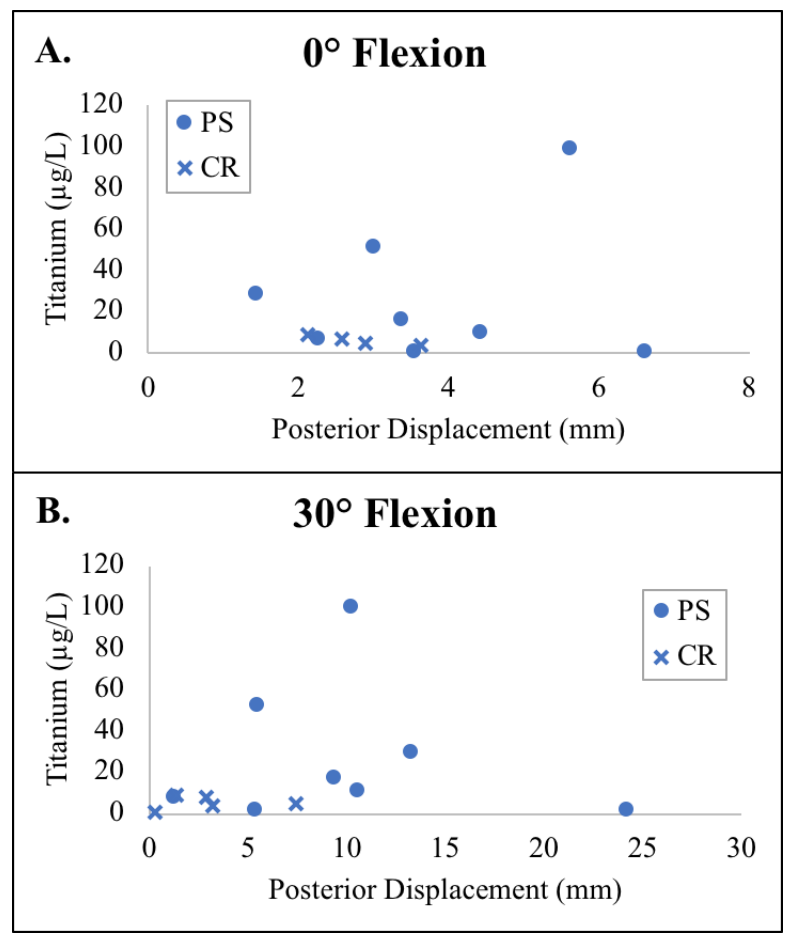

Figure B-23. Relationship between posterior displacement at (A) $0^{\circ}$ and (B) $30^{\circ}$ of flexion and $T i$ concentrations in periprosthetic tissue 


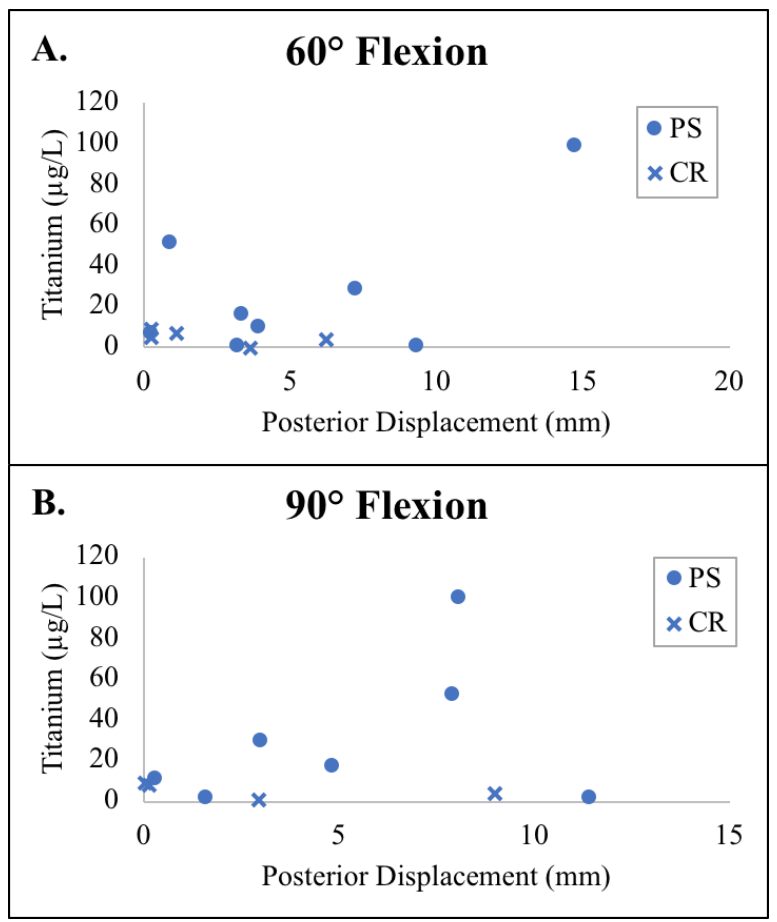

Figure B-24. Relationship between posterior displacement at (A) $60^{\circ}$ and (B) $90^{\circ}$ of flexion and $\mathrm{Ti}$ concentrations in periprosthetic tissue 


\section{APPENDIX C. CORRELATIONS BETWEEN LAXITY AND INFLAMMATORY CYTOKINES}

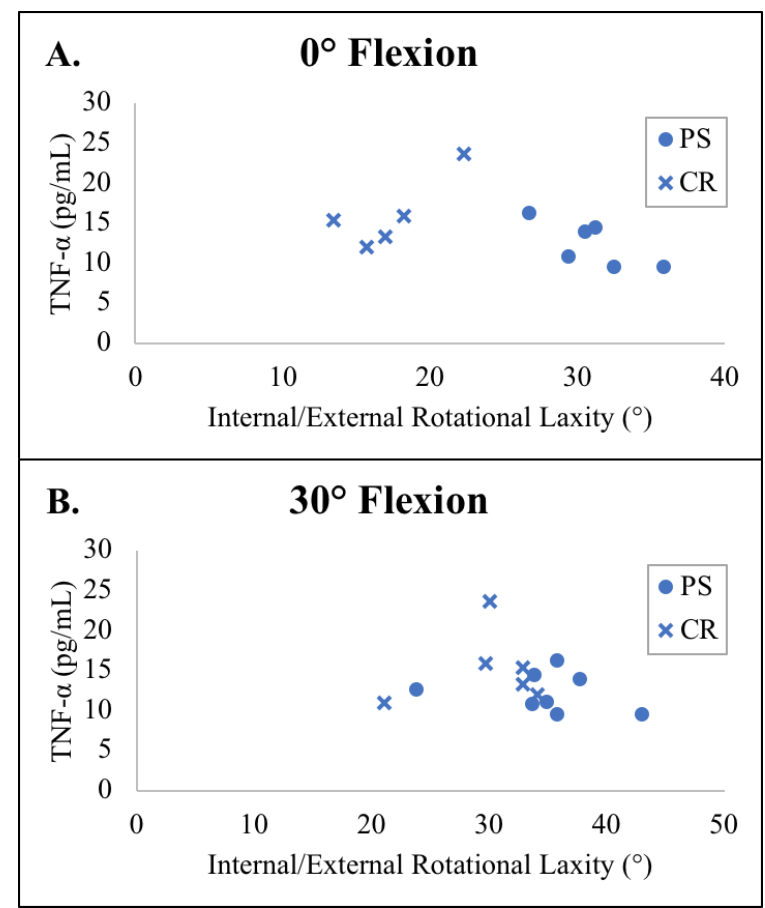

Figure C-1. Relationship between IE rotational laxity at (A) $0^{\circ}$ and (B) $30^{\circ}$ of flexion and TNF- $\alpha$ concentrations in synovial fluid 


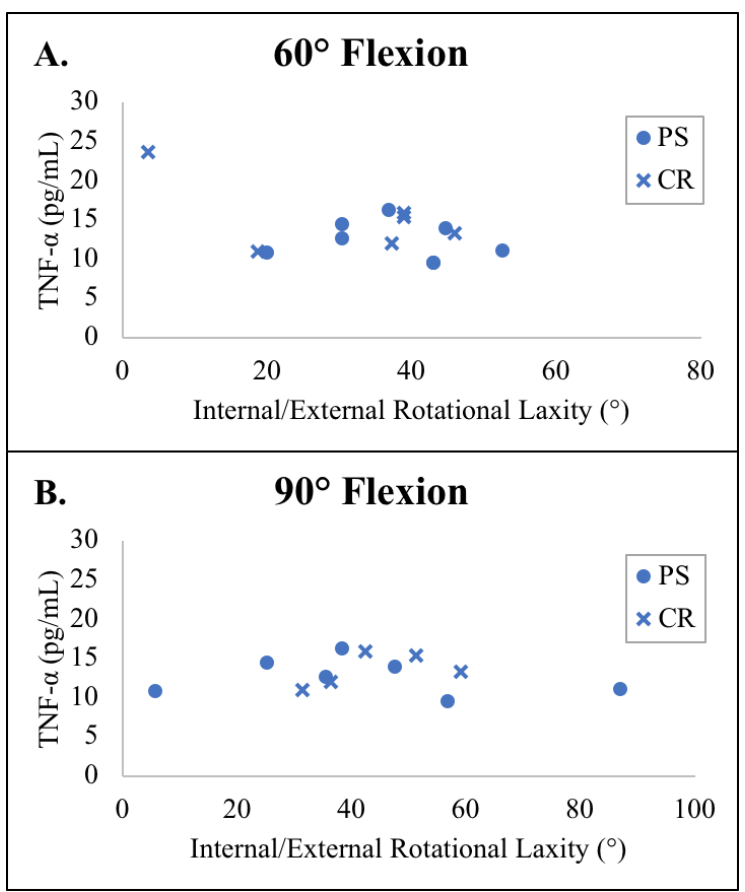

Figure C-2. Relationship between IE rotational laxity at (A) $60^{\circ}$ and (B) $90^{\circ}$ of flexion and TNF- $\alpha$ concentrations in synovial fluid

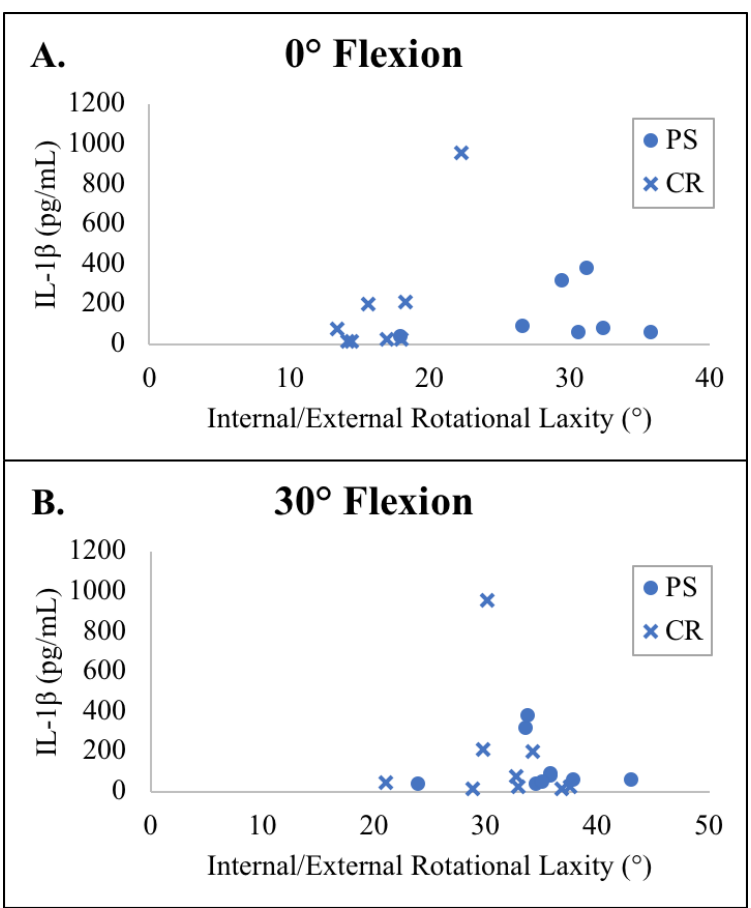

Figure C-3. Relationship between IE rotational laxity at (A) $0^{\circ}$ and (B) $30^{\circ}$ of flexion and IL-1 $\beta$ concentrations in synovial fluid 


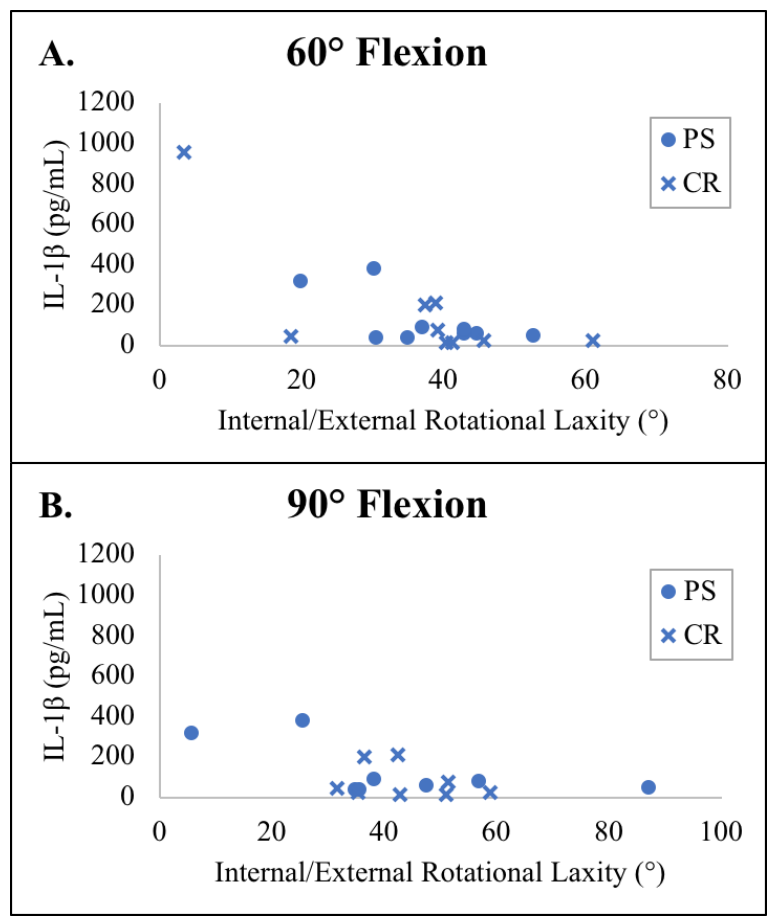

Figure C-4. Relationship between IE rotational laxity at (A) $60^{\circ}$ and (B) $90^{\circ}$ of flexion and IL-1 $\beta$ concentrations in synovial fluid

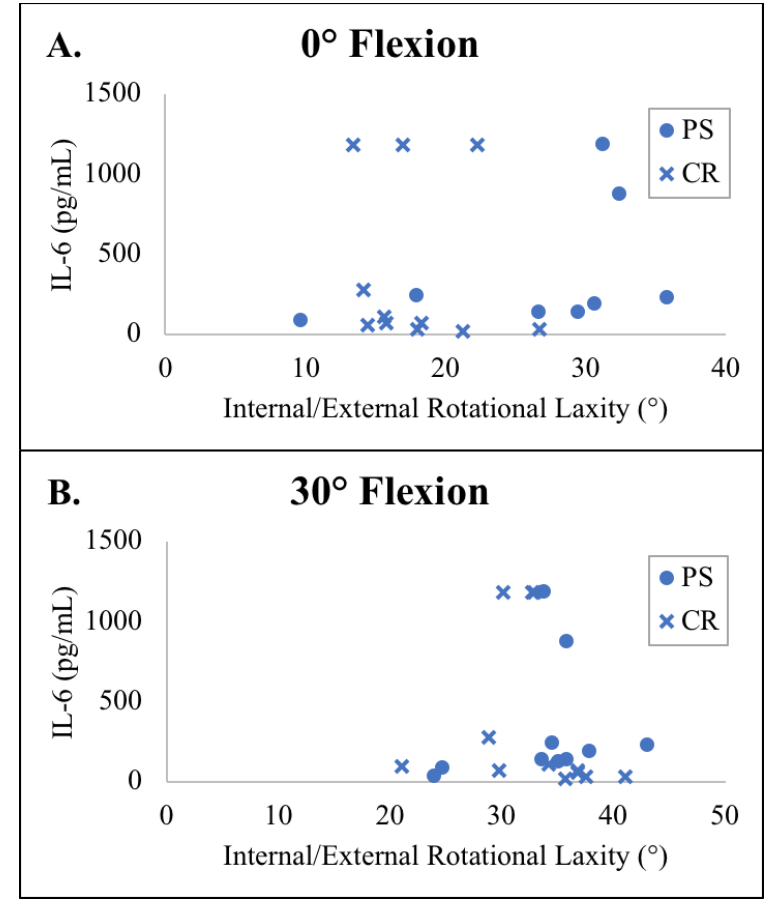

Figure C-5. Relationship between IE rotational laxity at (A) $0^{\circ}$ and (B) $30^{\circ}$ of flexion and IL-6 concentrations in synovial fluid 


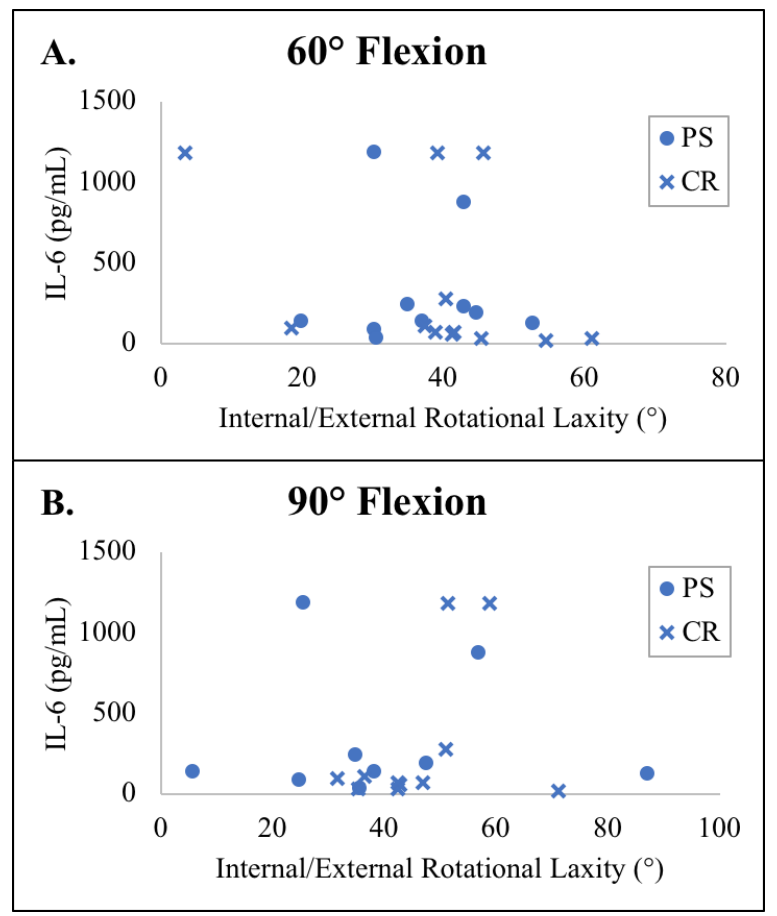

Figure C-6. Relationship between IE rotational laxity at (A) $60^{\circ}$ and (B) $90^{\circ}$ of flexion and IL-6 concentrations in synovial fluid

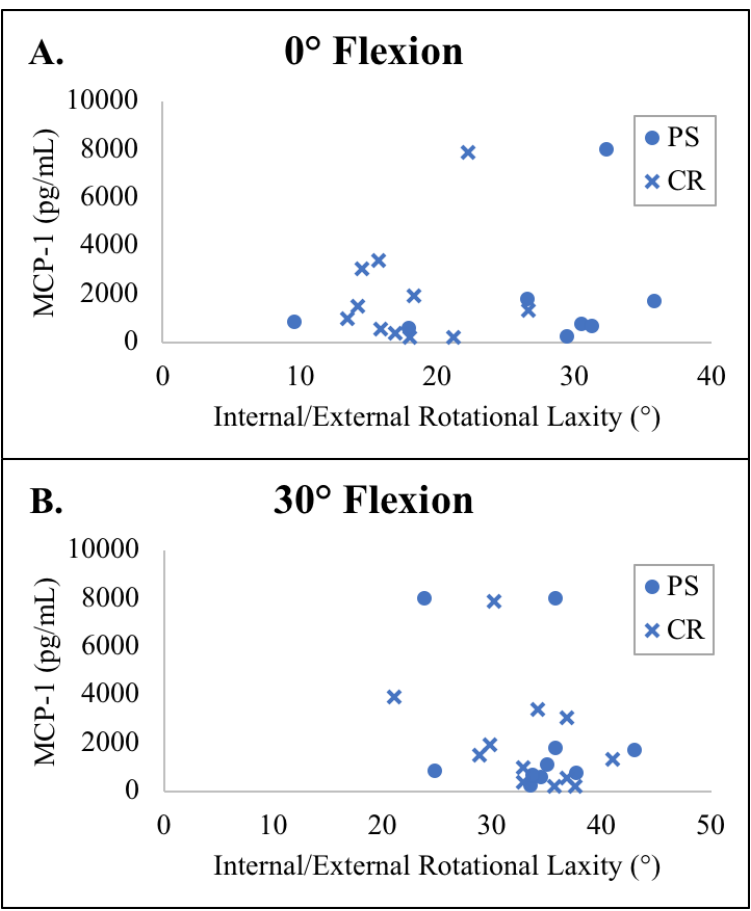

Figure C-7. Relationship between IE rotational laxity at (A) $0^{\circ}$ and (B) $30^{\circ}$ of flexion and MCP-1 concentrations in synovial fluid 


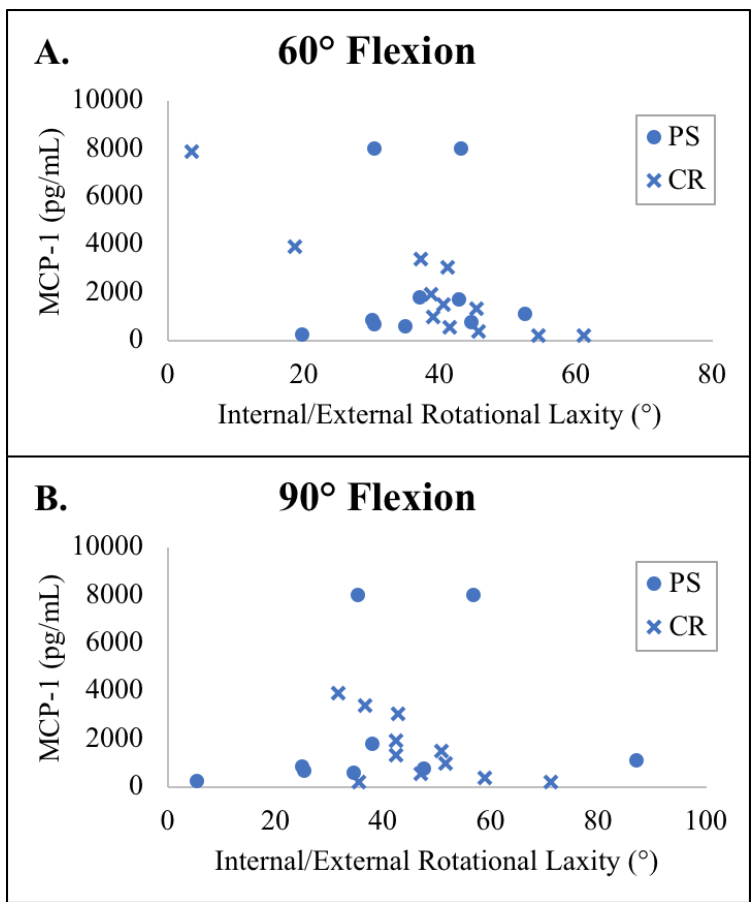

Figure C-8. Relationship between IE rotational laxity at (A) $60^{\circ}$ and (B) $90^{\circ}$ of flexion and MCP-1 concentrations in synovial fluid

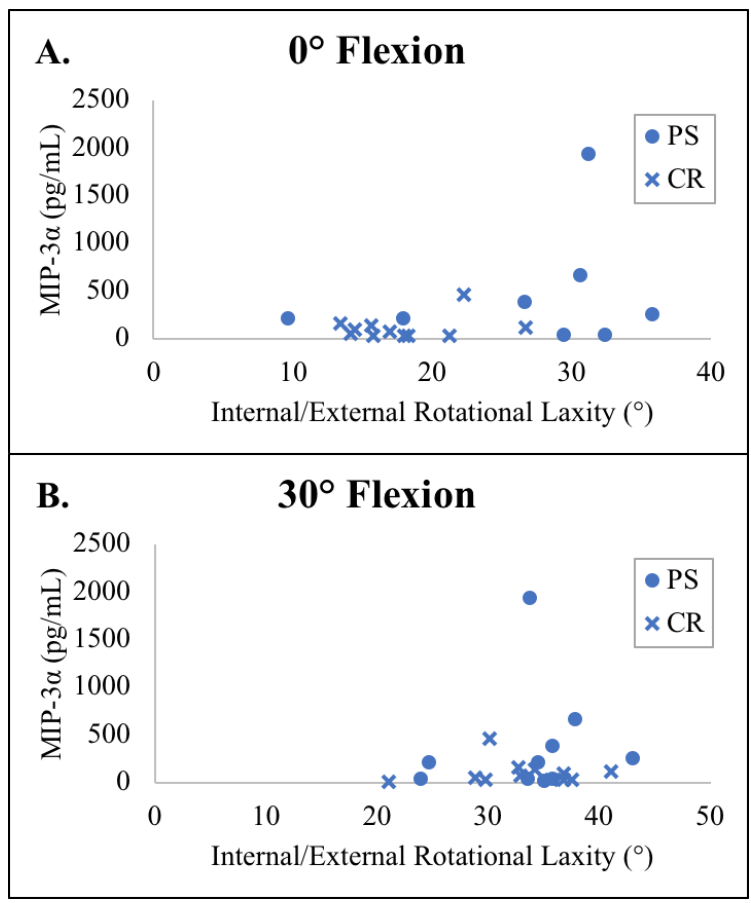

Figure C-9. Relationship between IE rotational laxity at (A) $0^{\circ}$ and (B) $30^{\circ}$ of flexion and MIP-3 $\alpha$ concentrations in synovial fluid 


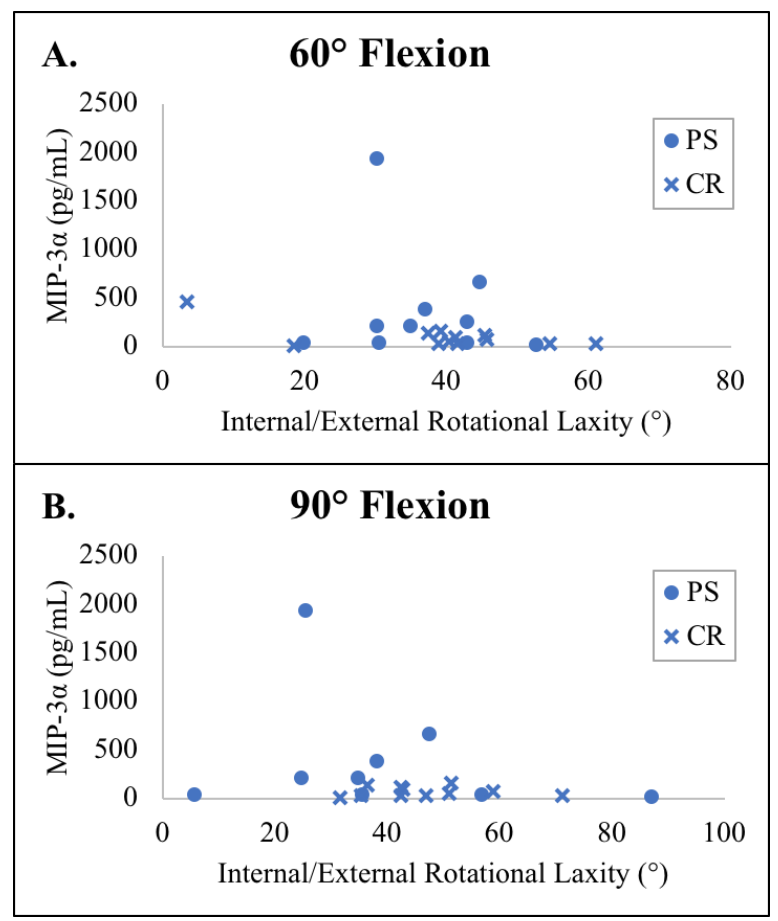

Figure C-10. Relationship between IE rotational laxity at (A) $60^{\circ}$ and (B) $90^{\circ}$ of flexion and MIP-3 $\alpha$ concentrations in synovial fluid

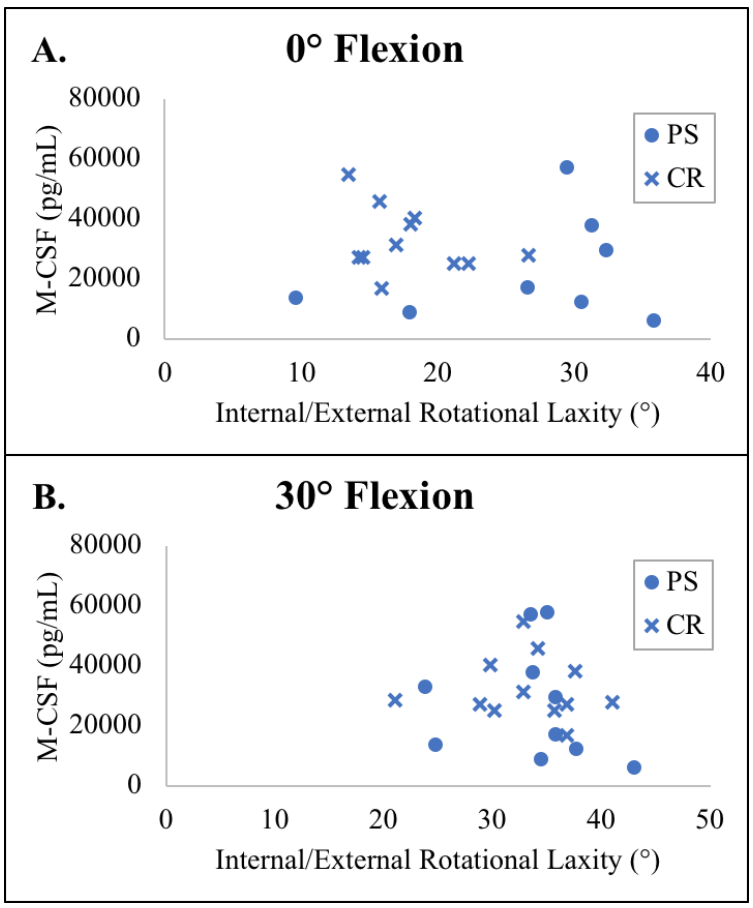

Figure C-11. Relationship between IE rotational laxity at (A) $0^{\circ}$ and (B) $30^{\circ}$ of flexion and M-CSF concentrations in synovial fluid 


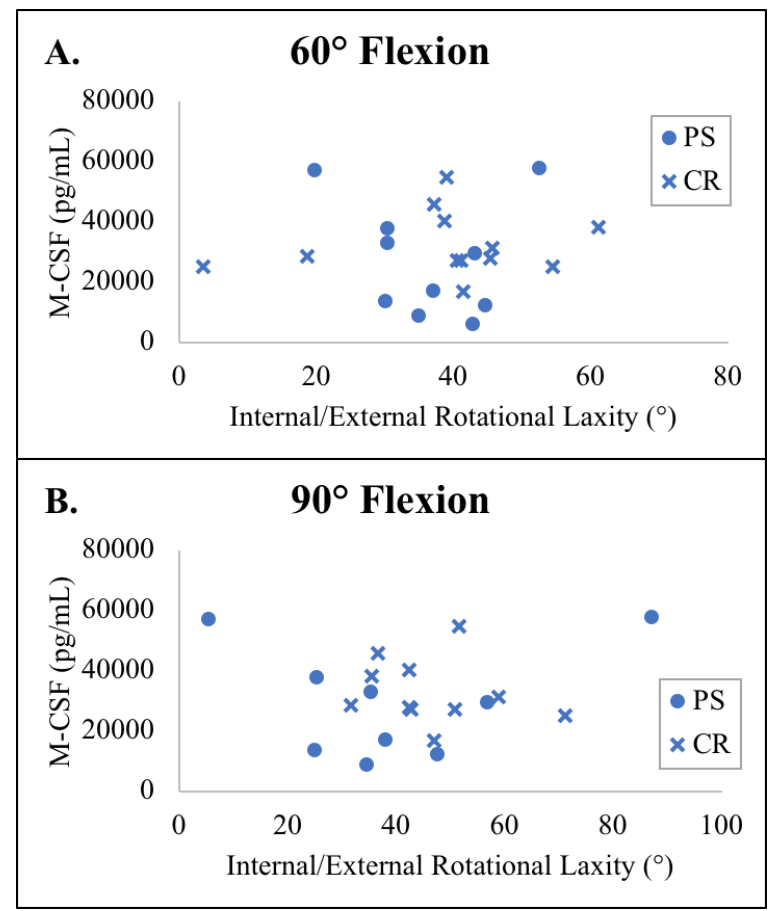

Figure C-12. Relationship between IE rotational laxity at (A) $60^{\circ}$ and (B) $90^{\circ}$ of flexion and M-CSF concentrations in synovial fluid

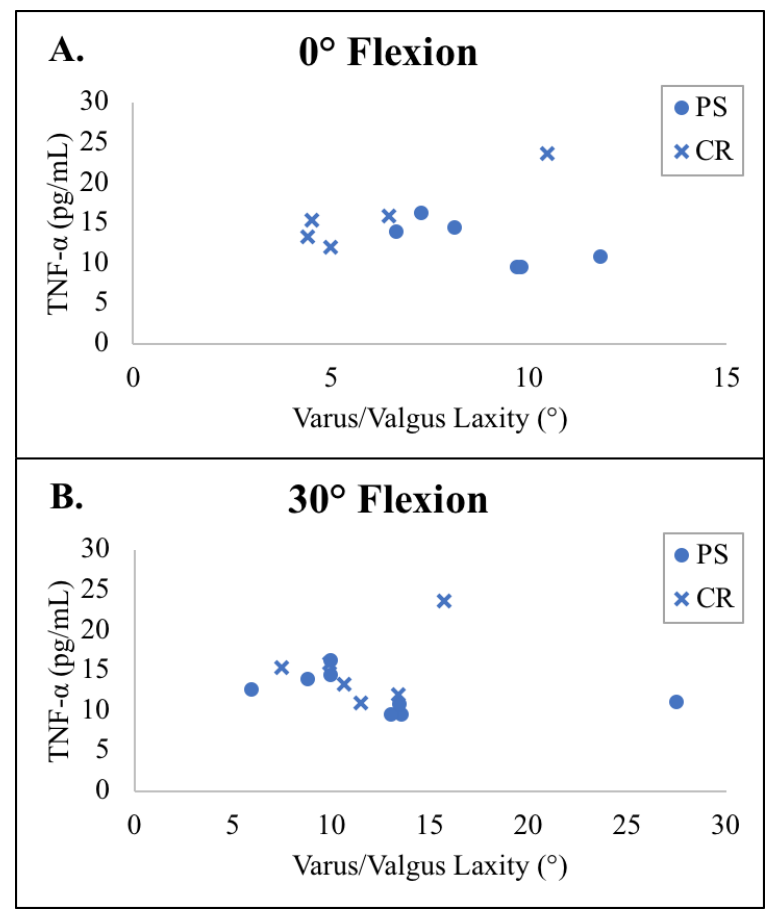

Figure C-13. Relationship between VV laxity at (A) $0^{\circ}$ and (B) $30^{\circ}$ of flexion and TNF- $\alpha$ concentrations in synovial fluid 


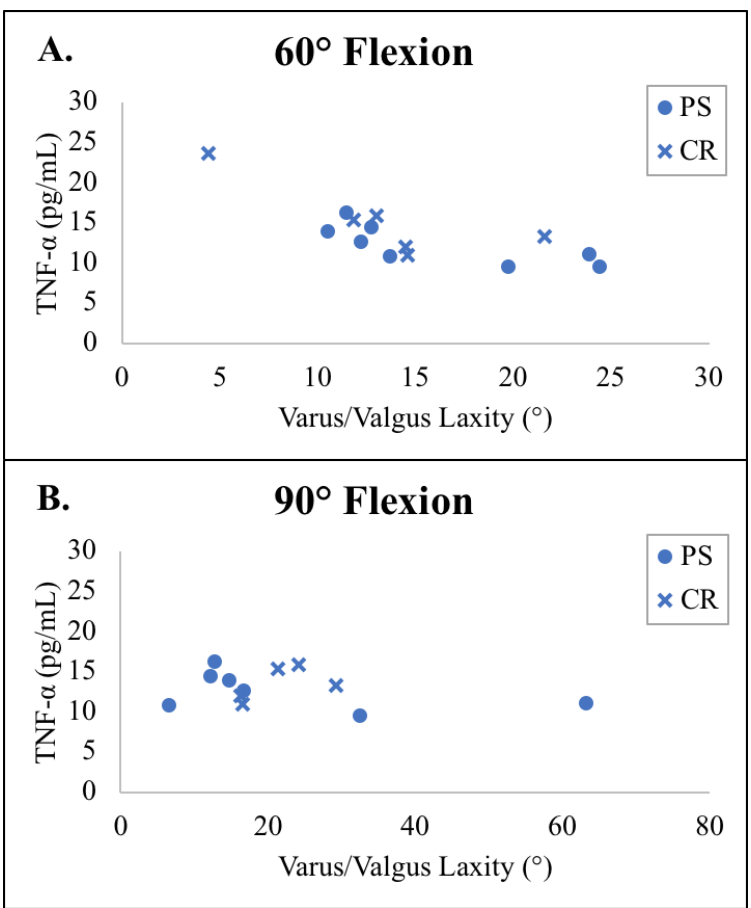

Figure C-14. Relationship between VV laxity at (A) $60^{\circ}$ and (B) $90^{\circ}$ of flexion and TNF- $\alpha$ concentrations in synovial fluid

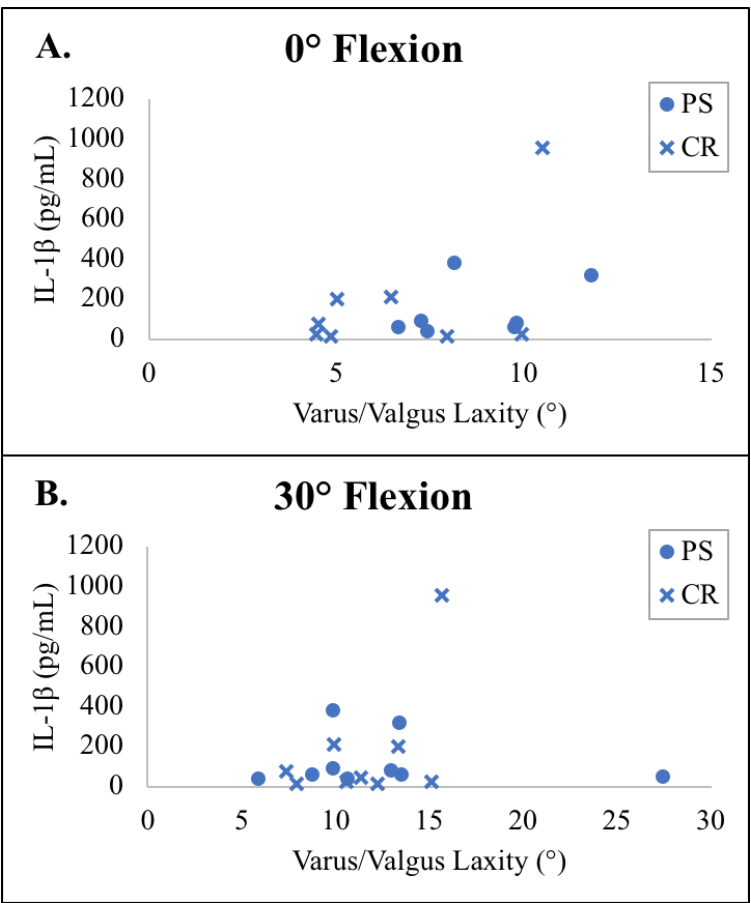

Figure C-15. Relationship between VV laxity at (A) $0^{\circ}$ and (B) $30^{\circ}$ of flexion and IL-1 $\beta$ concentrations in synovial fluid 


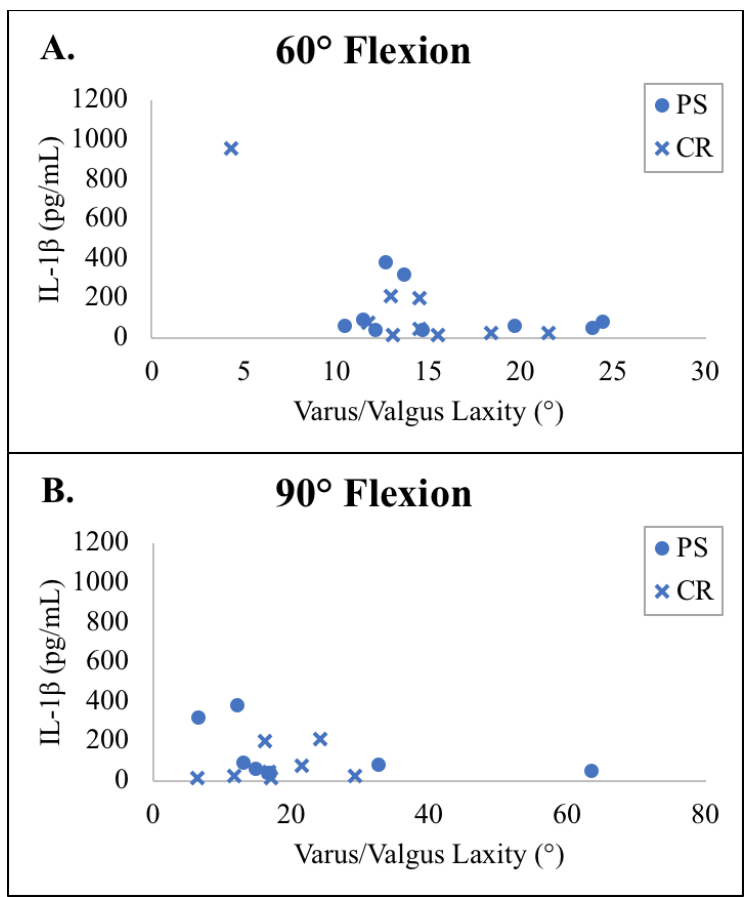

Figure C-16. Relationship between VV laxity at (A) $60^{\circ}$ and (B) $90^{\circ}$ of flexion and IL-1 $\beta$ concentrations in synovial fluid

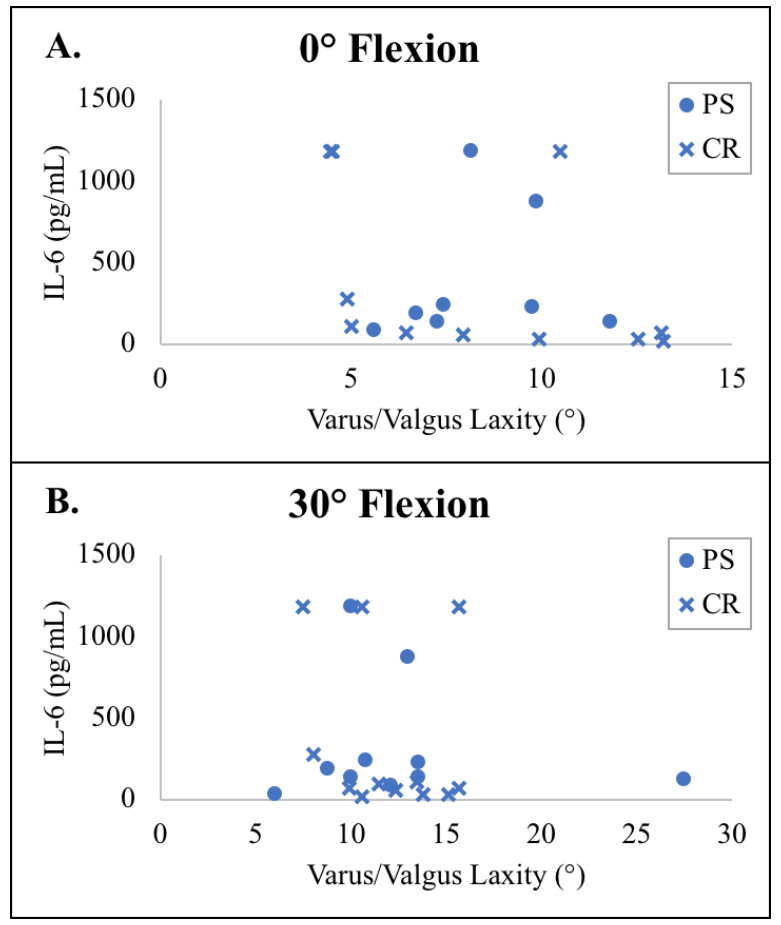

Figure C-17. Relationship between VV laxity at (A) $0^{\circ}$ and (B) $30^{\circ}$ of flexion and IL-6 concentrations in synovial fluid 


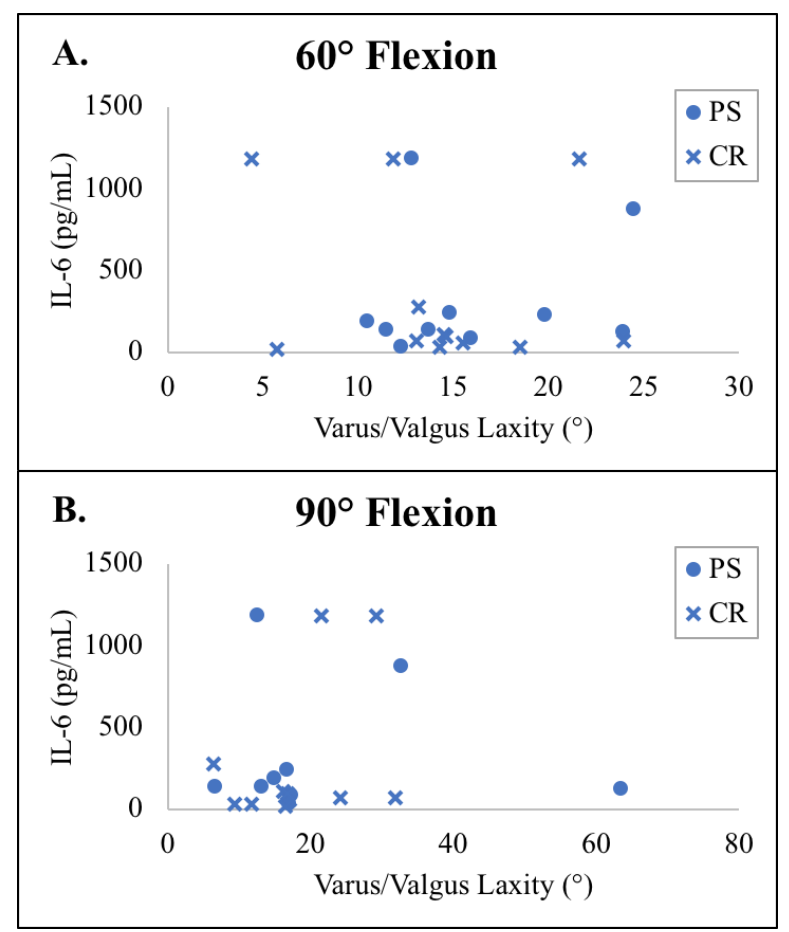

Figure C-18. Relationship between VV laxity at (A) $60^{\circ}$ and (B) $90^{\circ}$ of flexion and IL-6 concentrations in synovial fluid

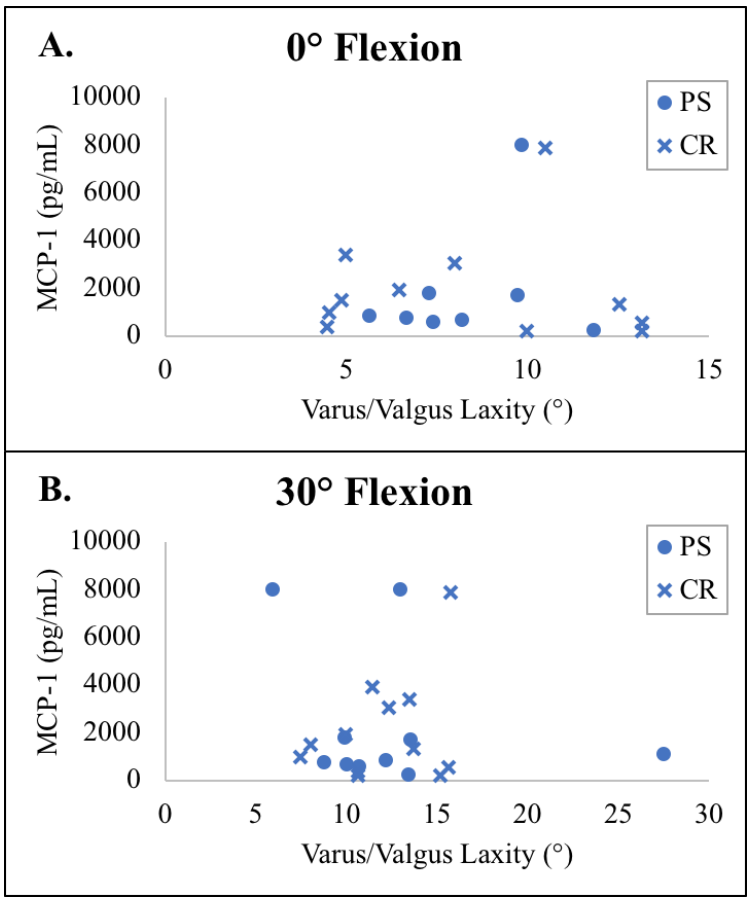

Figure C-19. Relationship between VV laxity at (A) $0^{\circ}$ and (B) $30^{\circ}$ of flexion and MCP-1 concentrations in synovial fluid 


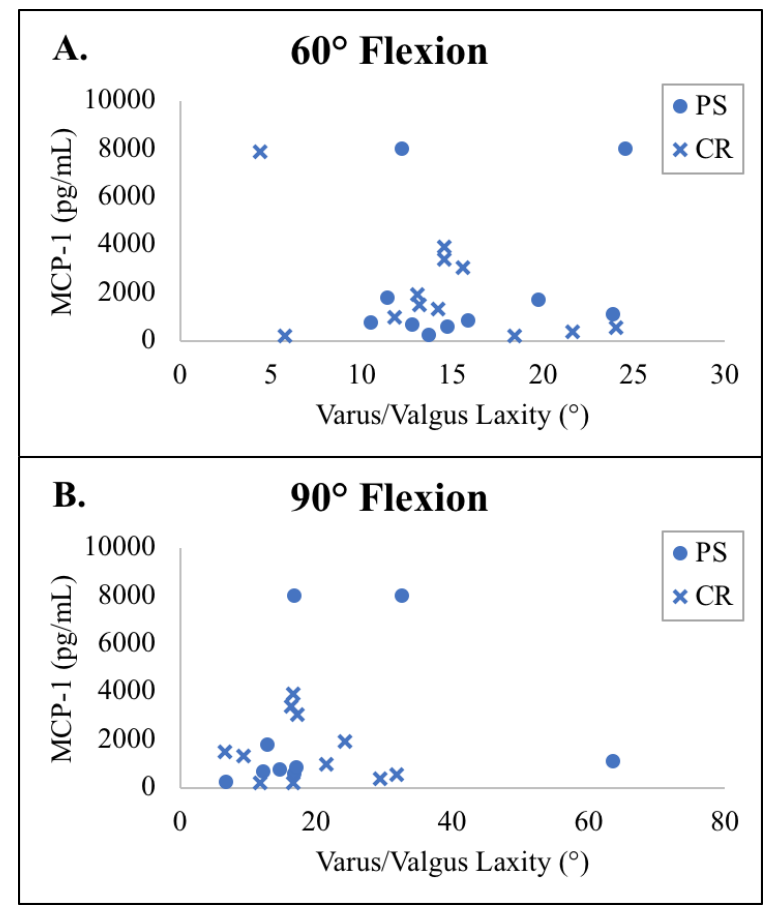

Figure C-20. Relationship between VV laxity at (A) $60^{\circ}$ and (B) $90^{\circ}$ of flexion and MCP-1 concentrations in synovial fluid

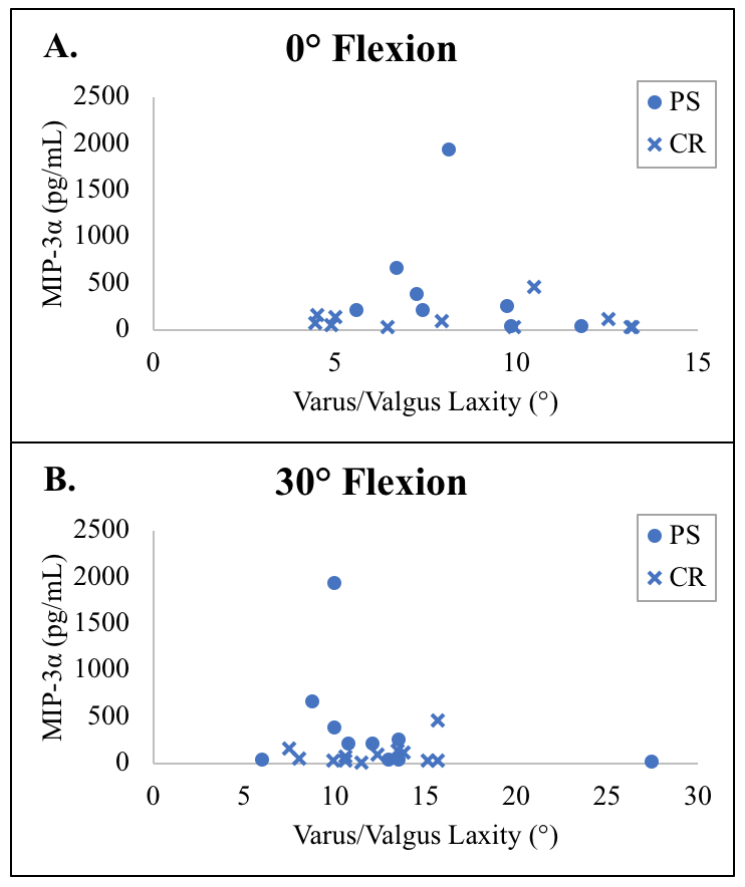

Figure C-21. Relationship between VV laxity at (A) $0^{\circ}$ and (B) $30^{\circ}$ of flexion and MIP-3 $\alpha$ concentrations in synovial fluid 


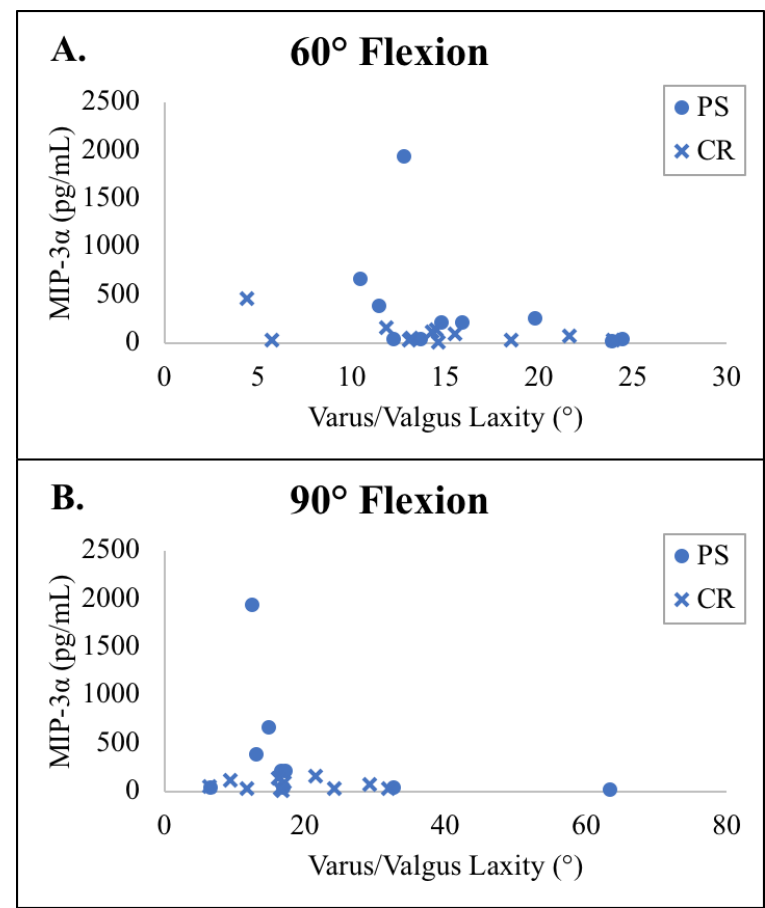

Figure C-22. Relationship between VV laxity at (A) $60^{\circ}$ and (B) $90^{\circ}$ of flexion and MIP-3 $\alpha$ concentrations in synovial fluid

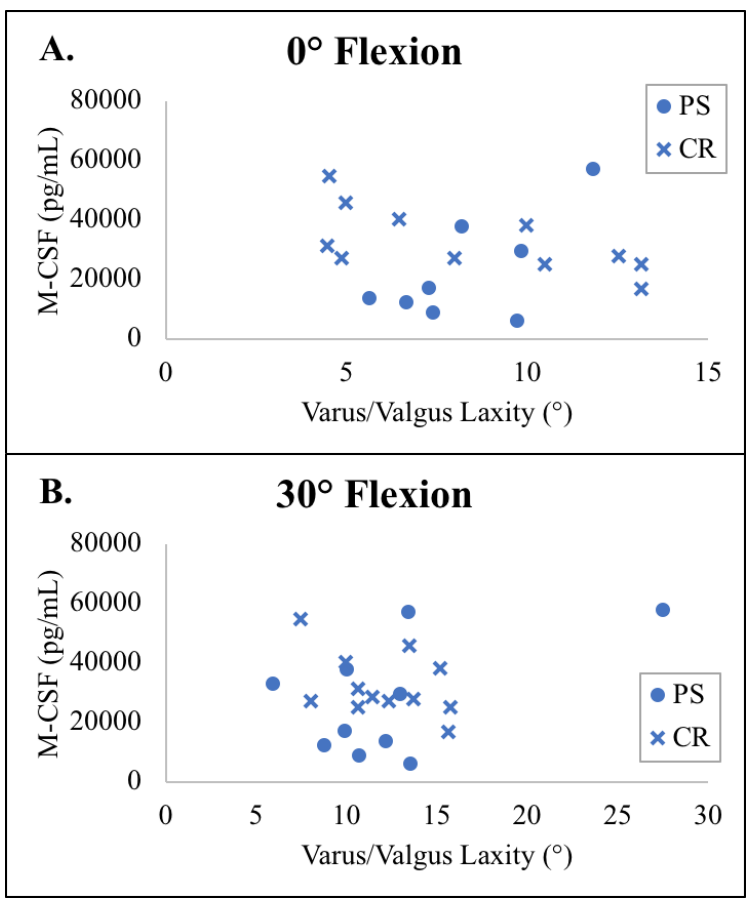

Figure C-23. Relationship between VV laxity at (A) $0^{\circ}$ and (B) $30^{\circ}$ of flexion and M-CSF concentrations in synovial fluid 


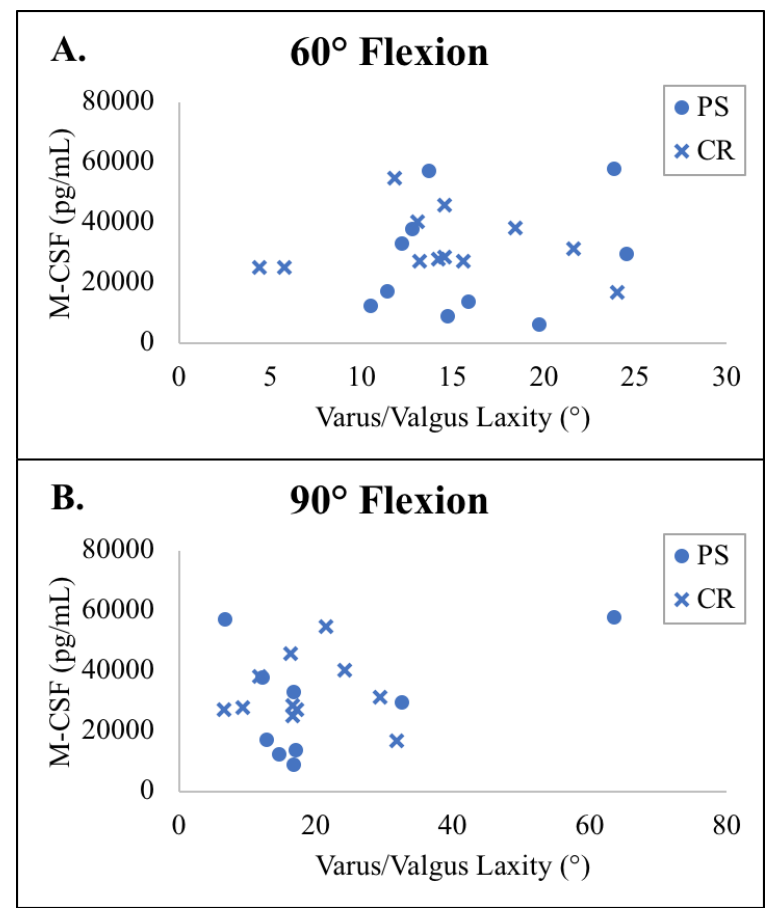

Figure C-24. Relationship between VV laxity at (A) $60^{\circ}$ and (B) $90^{\circ}$ of flexion and M-CSF concentrations in synovial fluid

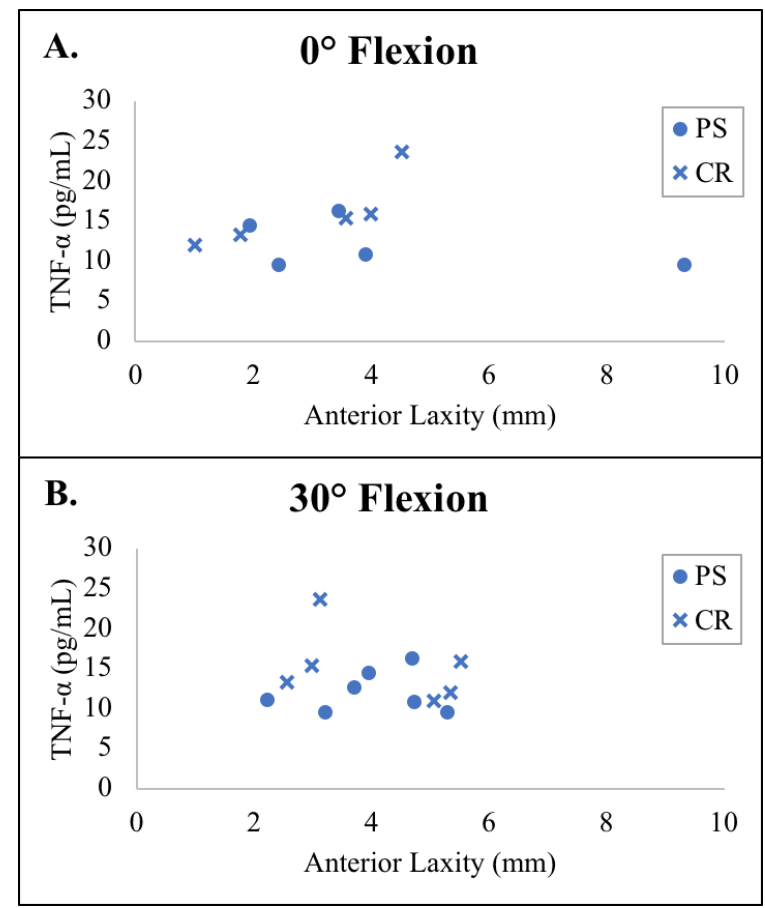

Figure C-25. Relationship between anterior laxity at (A) $0^{\circ}$ and (B) $30^{\circ}$ of flexion and TNF- $\alpha$ concentrations in synovial fluid 


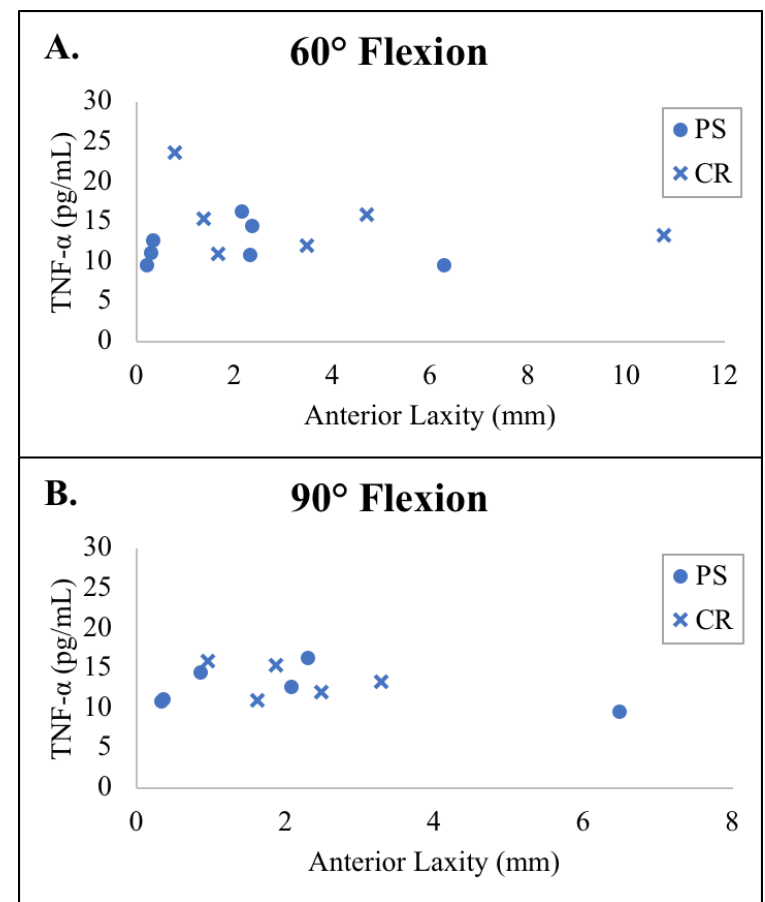

Figure C-26. Relationship between anterior laxity at (A) $60^{\circ}$ and (B) $90^{\circ}$ of flexion and $\mathrm{TNF}-\alpha$ concentrations in synovial fluid

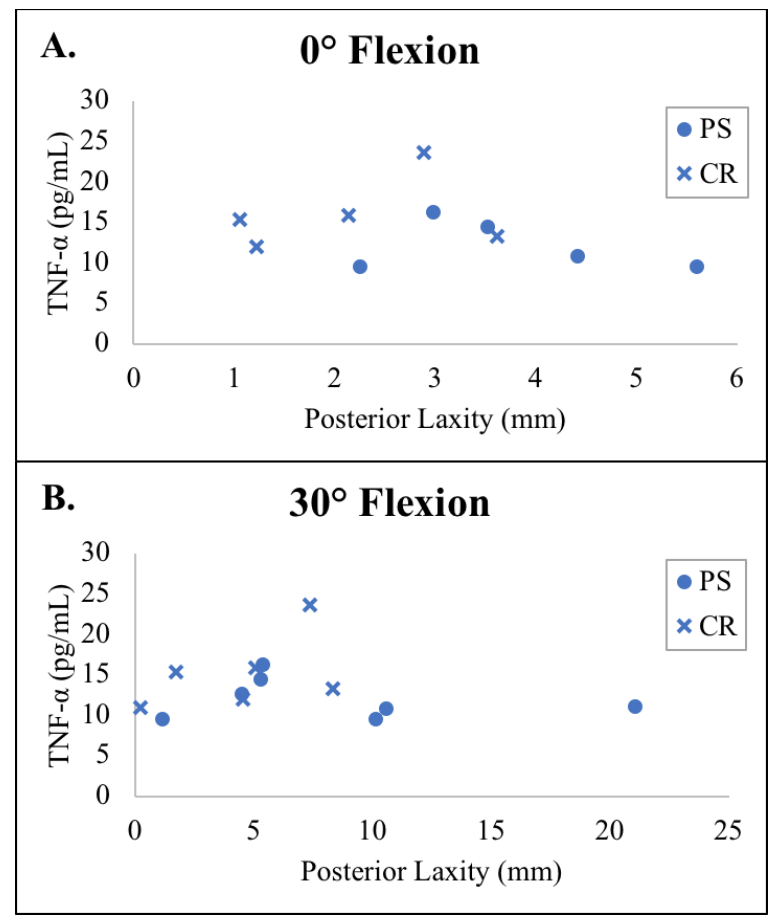

Figure C-27. Relationship between posterior laxity at (A) $0^{\circ}$ and (B) $30^{\circ}$ of flexion and TNF- $\alpha$ concentrations in synovial fluid 


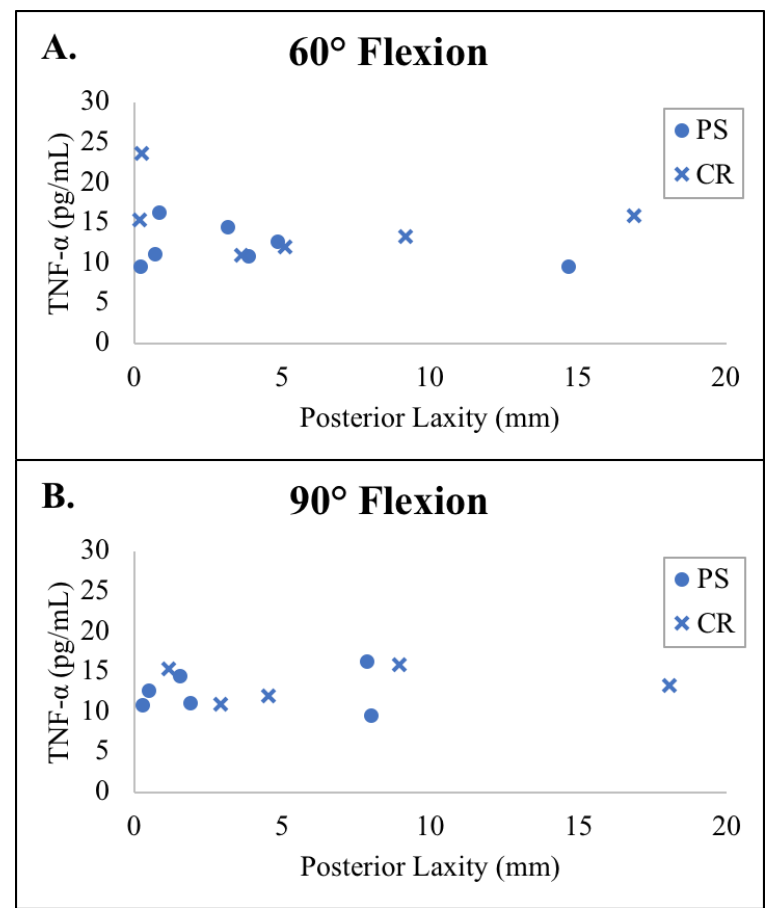

Figure C-28. Relationship between posterior laxity at (A) $60^{\circ}$ and (B) $90^{\circ}$ of flexion and $\mathrm{TNF}-\alpha$ concentrations in synovial fluid

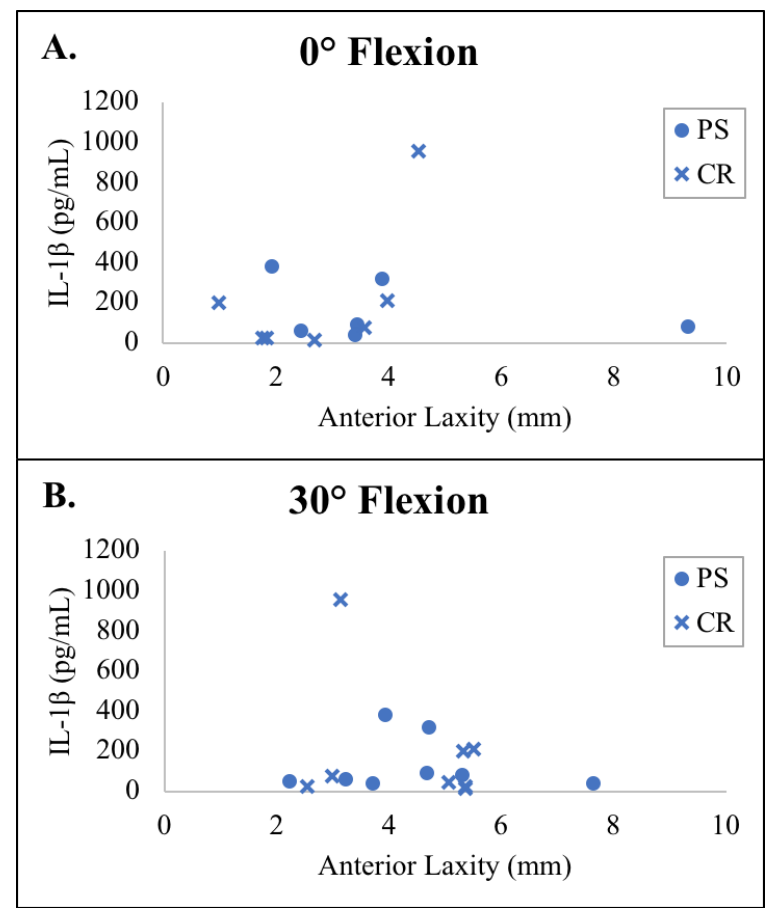

Figure C-29. Relationship between anterior laxity at (A) $0^{\circ}$ and (B) $30^{\circ}$ of flexion and $I L-1 \beta$ concentrations in synovial fluid 


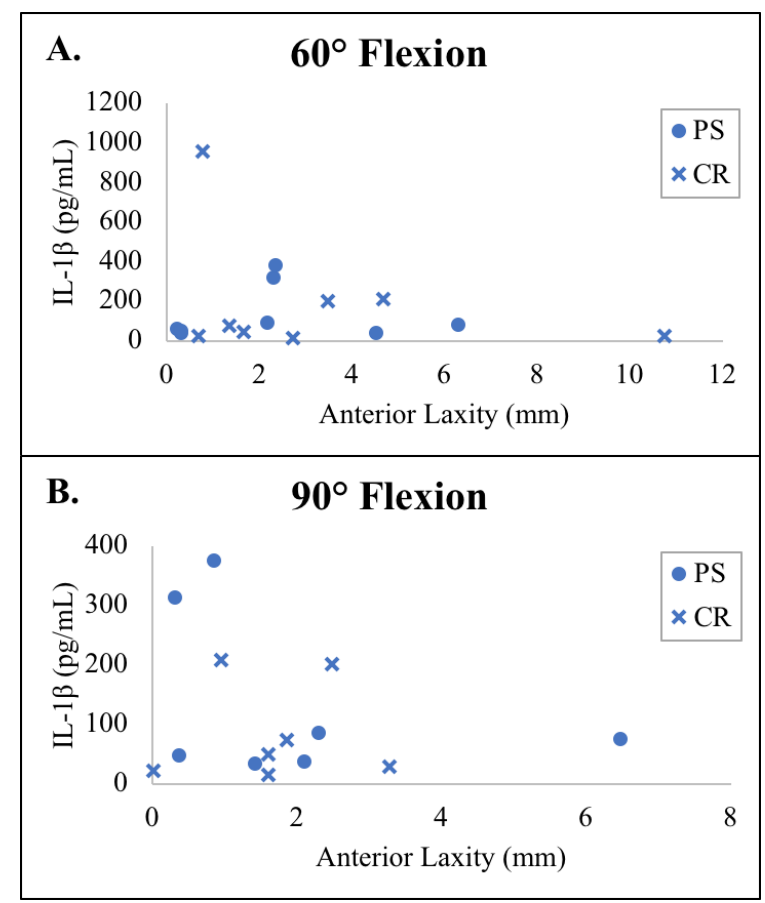

Figure C-30. Relationship between anterior laxity at (A) $60^{\circ}$ and (B) $90^{\circ}$ of flexion and IL-1 $\beta$ concentrations in synovial fluid

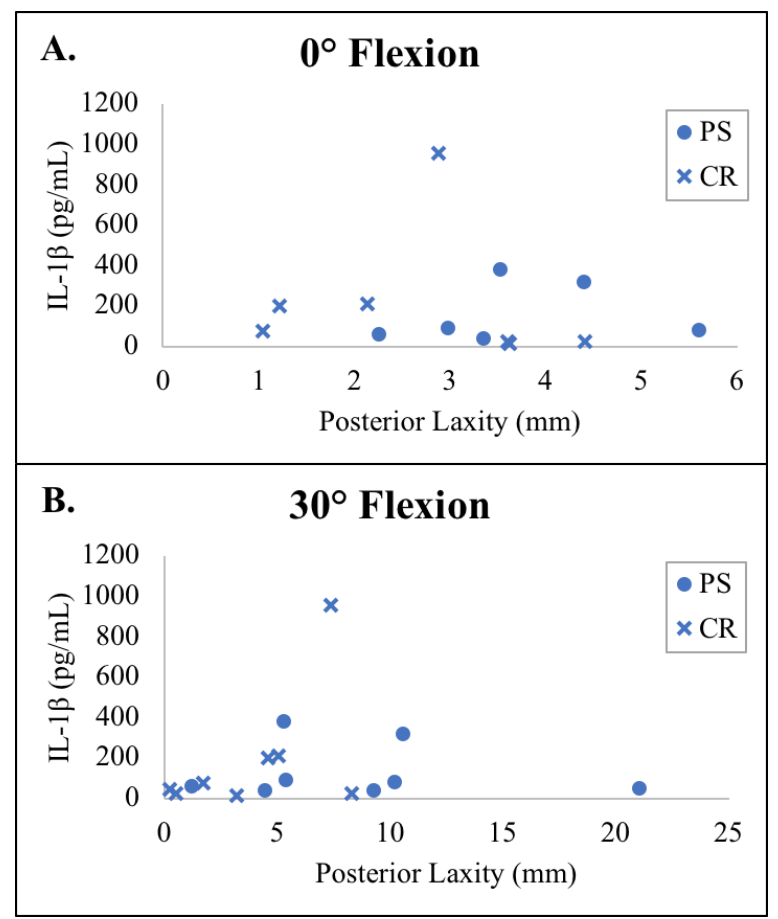

Figure C-31. Relationship between posterior laxity at (A) $0^{\circ}$ and (B) $30^{\circ}$ of flexion and IL-1 $\beta$ concentrations in synovial fluid 


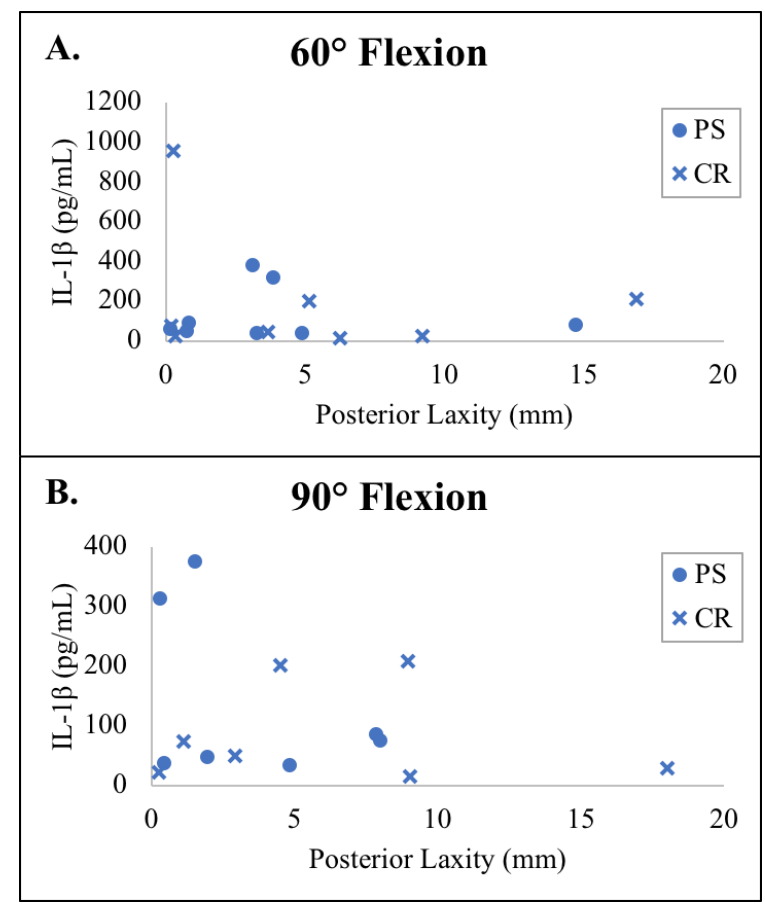

Figure C-32. Relationship between posterior laxity at (A) $60^{\circ}$ and (B) $90^{\circ}$ of flexion and IL-1 $\beta$ concentrations in synovial fluid

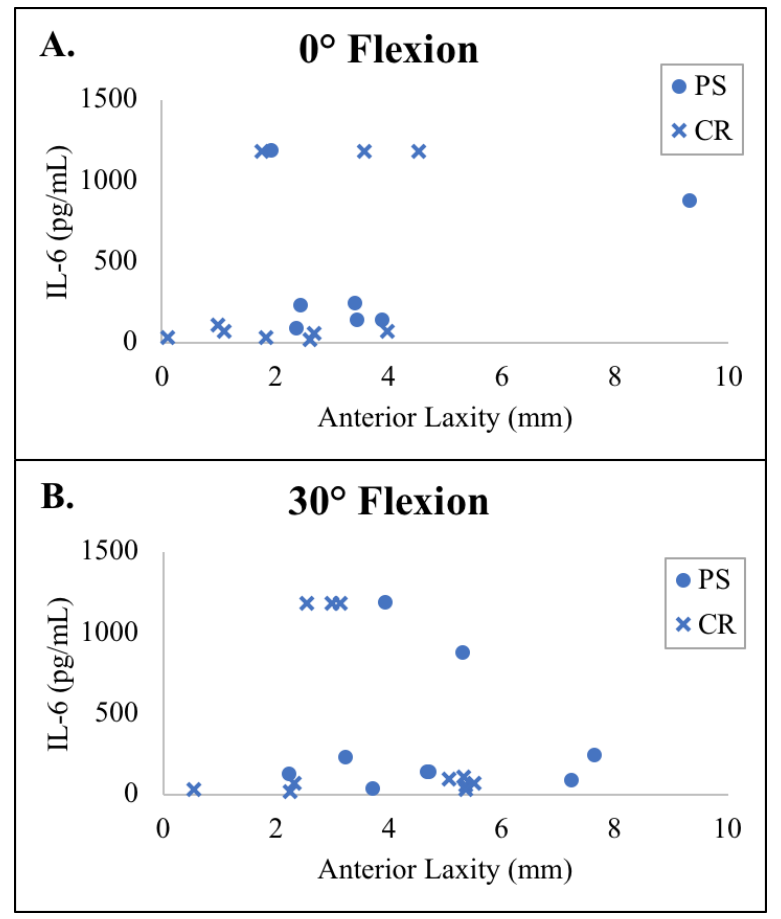

Figure C-33. Relationship between anterior laxity at (A) $0^{\circ}$ and (B) $30^{\circ}$ of flexion and IL-6 concentrations in synovial fluid 


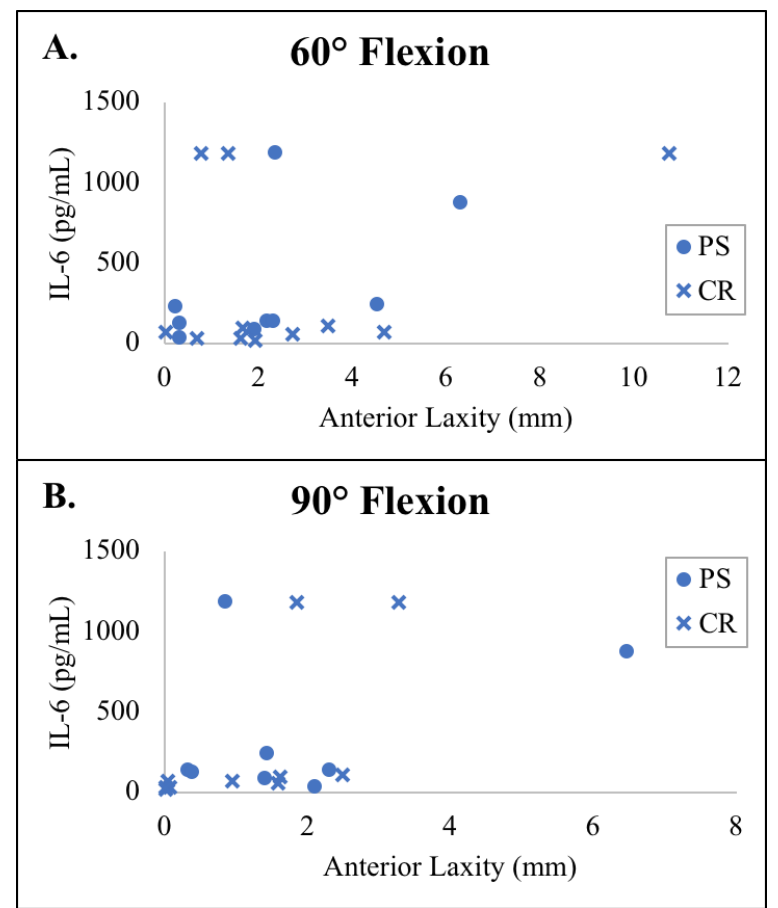

Figure C-34. Relationship between anterior laxity at (A) $60^{\circ}$ and (B) $90^{\circ}$ of flexion and IL-6 concentrations in synovial fluid

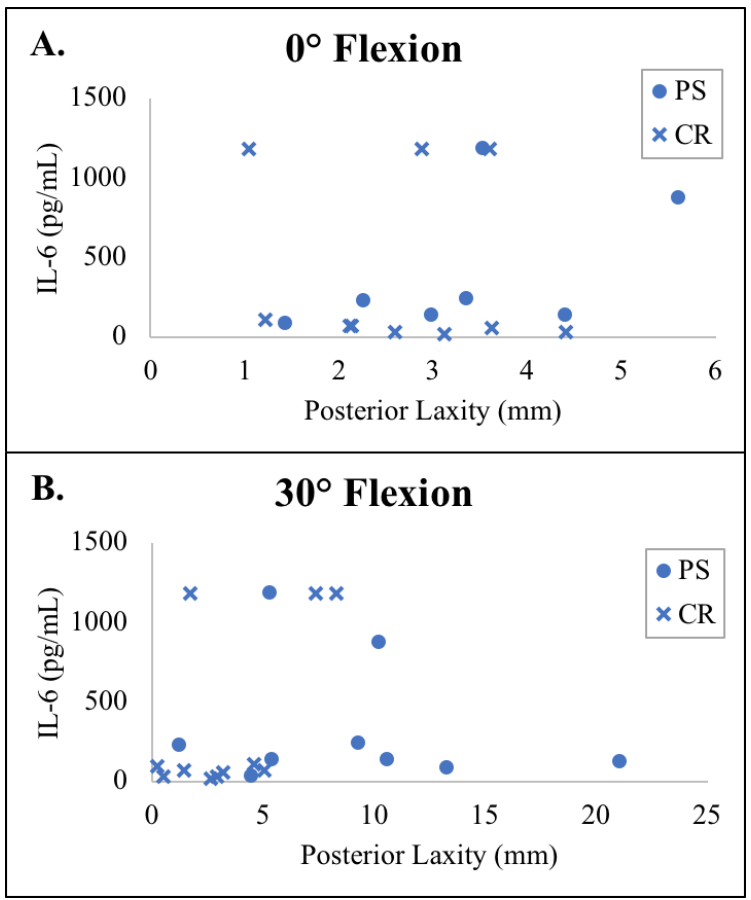

Figure C-35. Relationship between posterior laxity at (A) $0^{\circ}$ and (B) $30^{\circ}$ of flexion and IL-6 concentrations in synovial fluid 


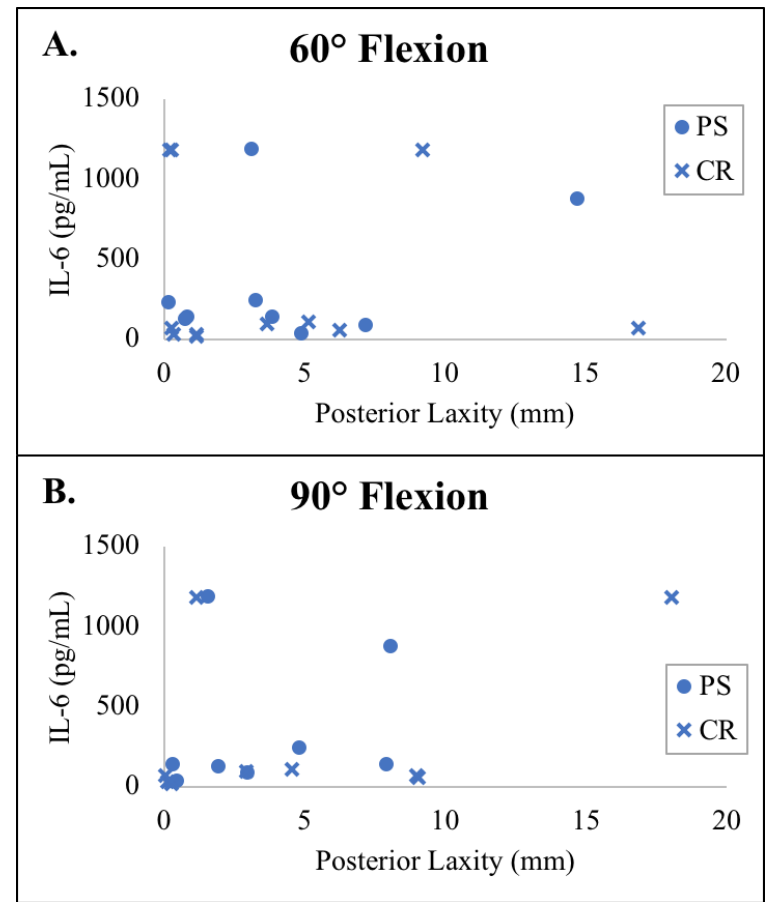

Figure C-36. Relationship between posterior laxity at (A) $60^{\circ}$ and (B) $90^{\circ}$ of flexion and IL-6 concentrations in synovial fluid

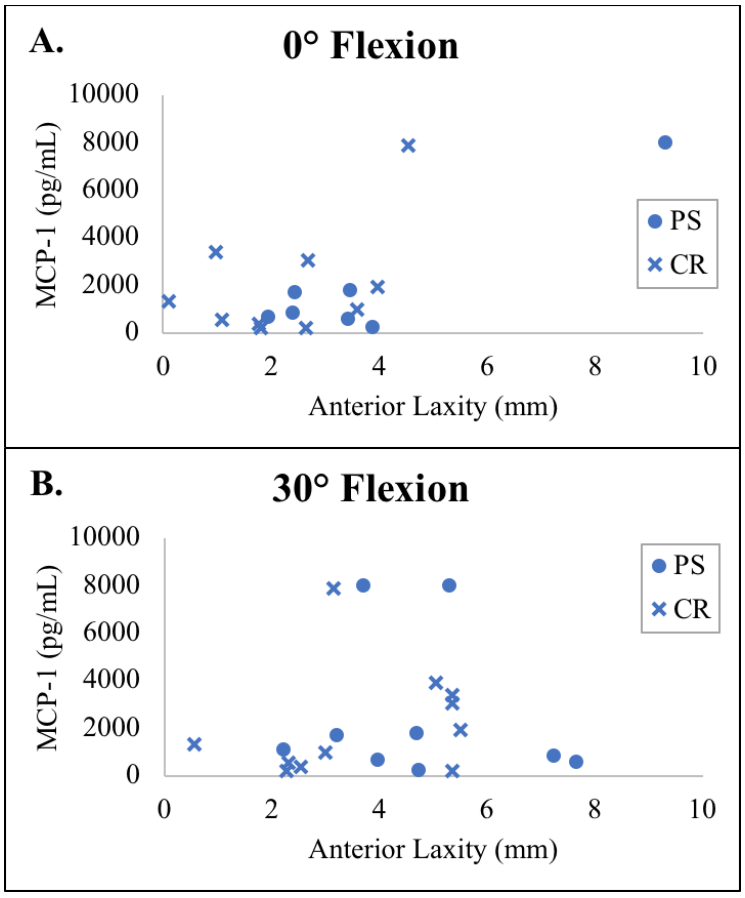

Figure C-37. Relationship between anterior laxity at (A) $0^{\circ}$ and (B) $30^{\circ}$ of flexion and MCP-1 concentrations in synovial fluid 


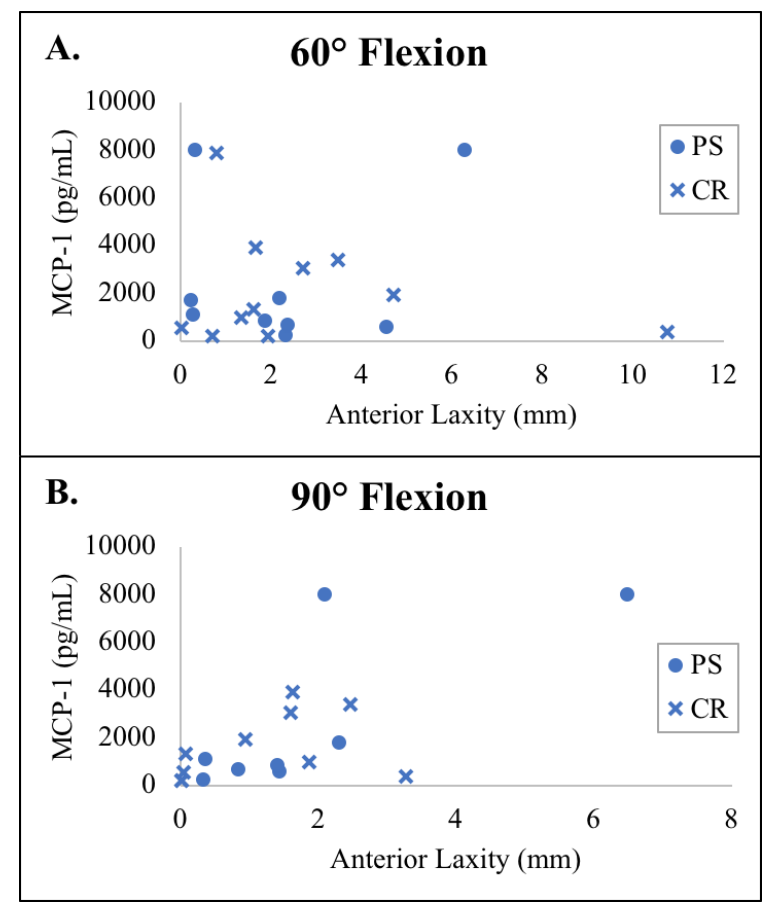

Figure C-38. Relationship between anterior laxity at (A) $60^{\circ}$ and (B) $90^{\circ}$ of flexion and MCP-1 concentrations in synovial fluid

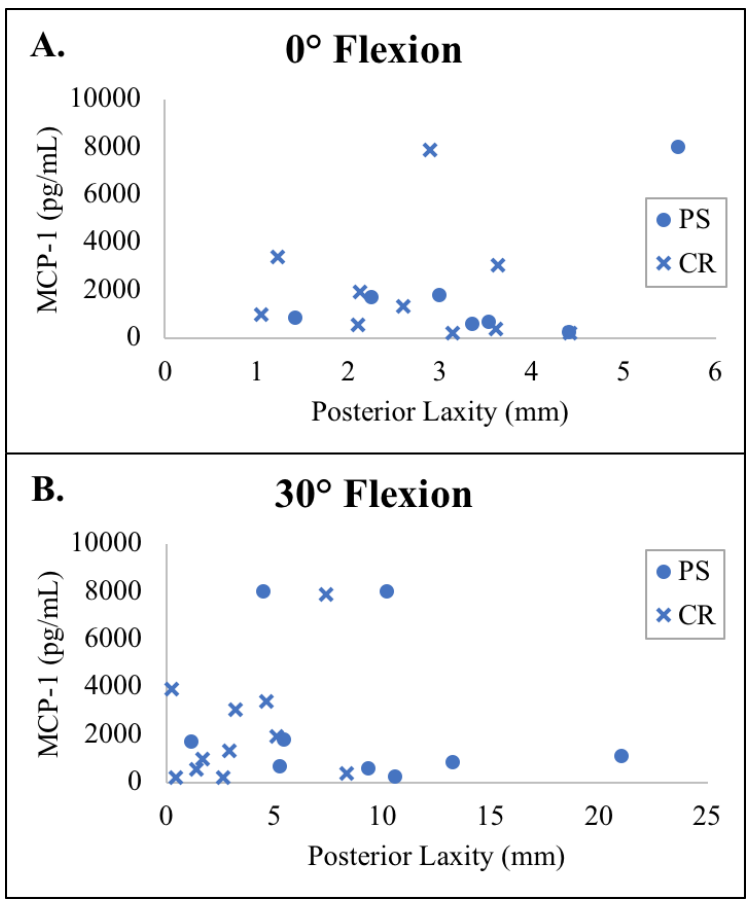

Figure C-39. Relationship between posterior laxity at (A) $0^{\circ}$ and (B) $30^{\circ}$ of flexion and MCP-1 concentrations in synovial fluid 


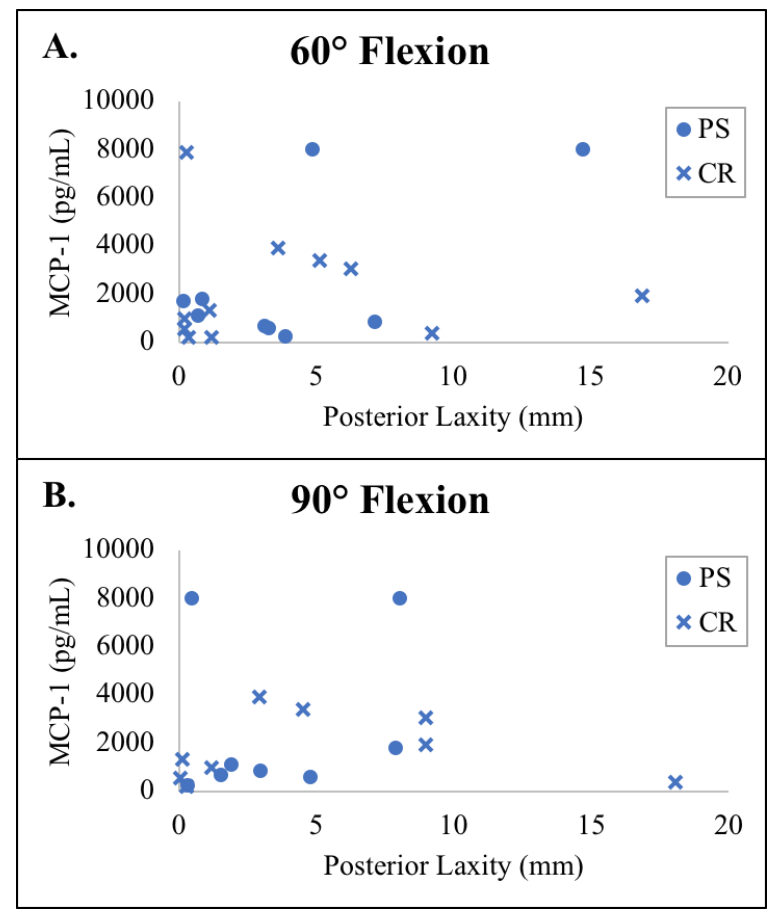

Figure C-40. Relationship between posterior laxity at (A) $60^{\circ}$ and (B) $90^{\circ}$ of flexion and MCP-1 concentrations in synovial fluid

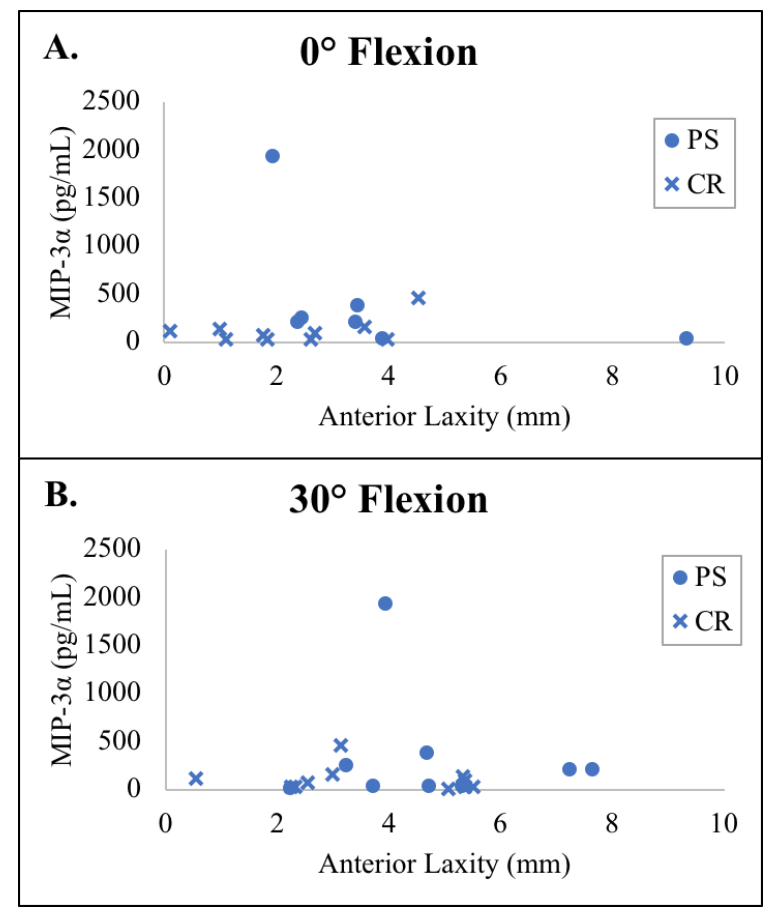

Figure C-41. Relationship between anterior laxity at (A) $0^{\circ}$ and (B) $30^{\circ}$ of flexion and MIP-3 $\alpha$ concentrations in synovial fluid 


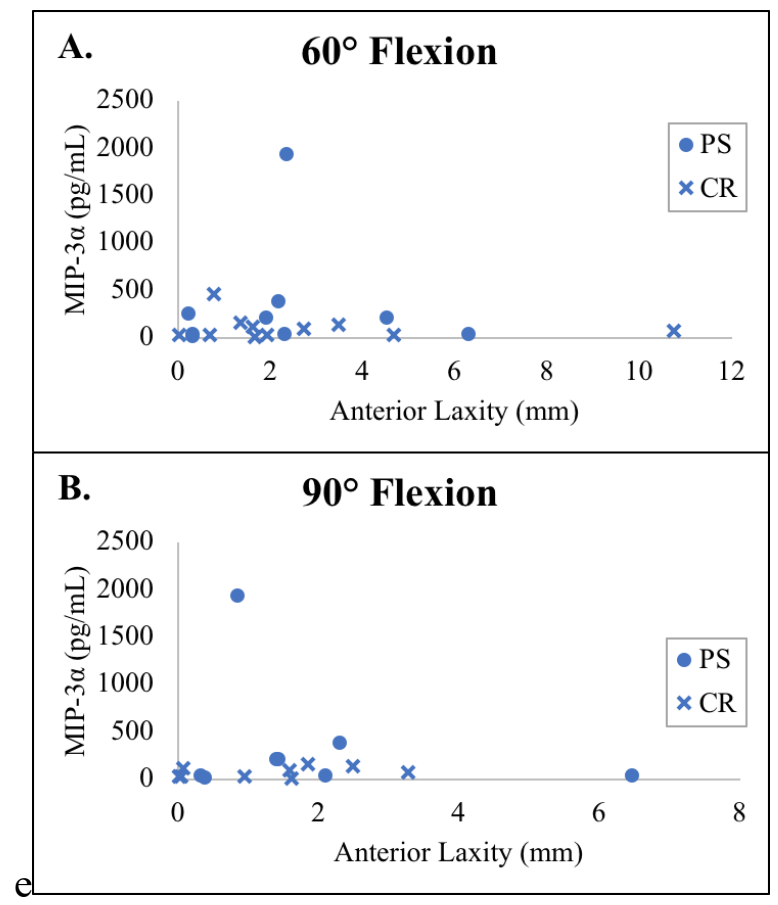

Figure C-42. Relationship between anterior laxity at (A) $60^{\circ}$ and (B) $90^{\circ}$ of flexion and MIP-3 $\alpha$ concentrations in synovial fluid

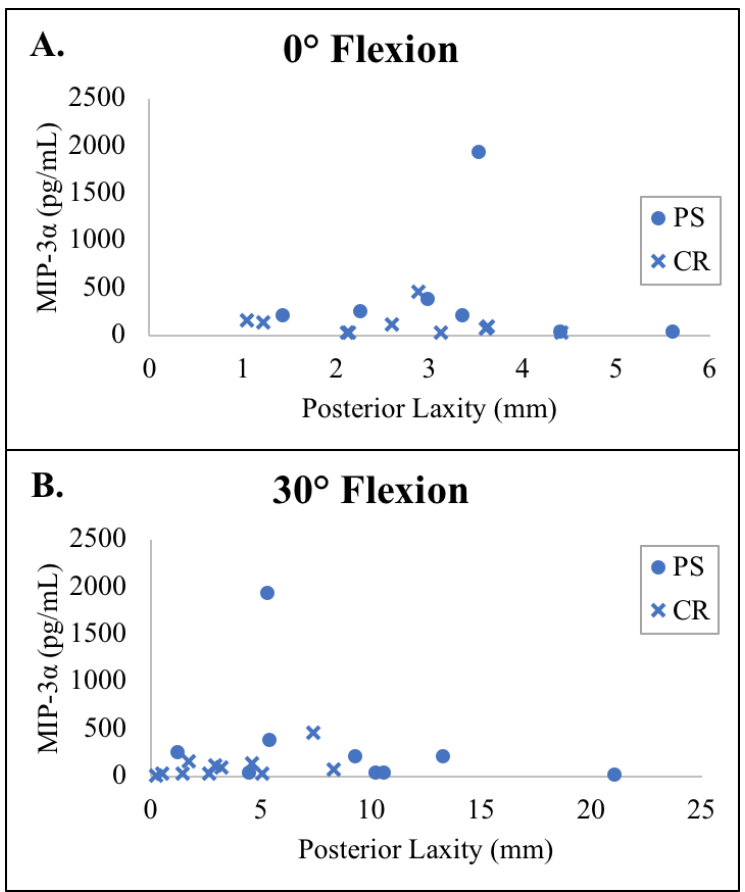

Figure C-43. Relationship between posterior laxity at (A) $0^{\circ}$ and (B) $30^{\circ}$ of flexion and MIP-3 $\alpha$ concentrations in synovial fluid 


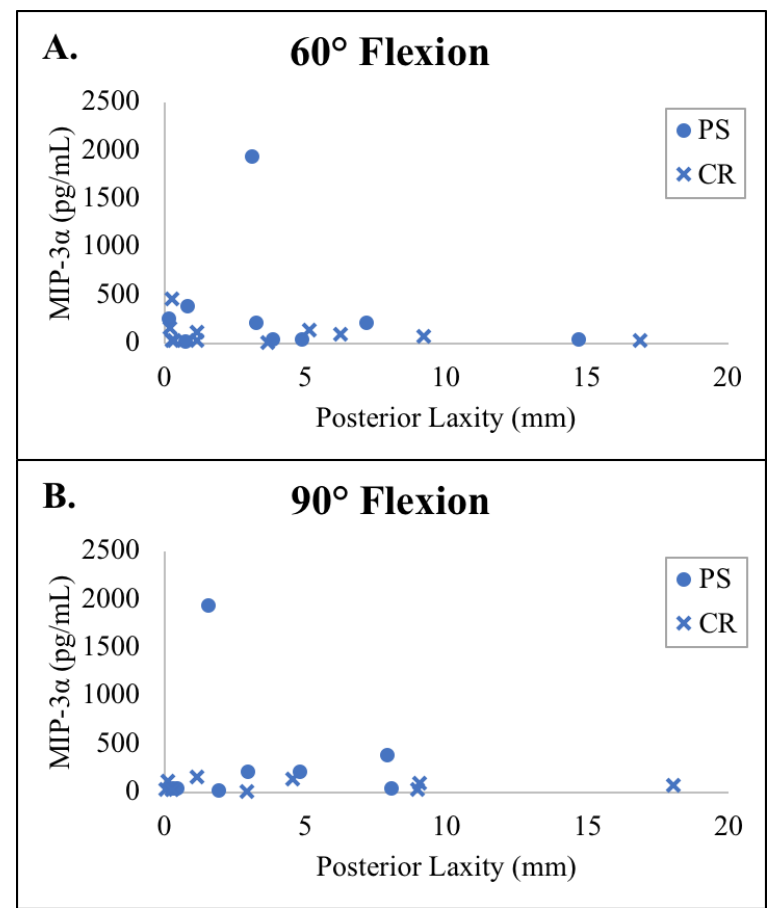

Figure C-44. Relationship between posterior laxity at (A) $60^{\circ}$ and (B) $90^{\circ}$ of flexion and MIP-3 $\alpha$ concentrations in synovial fluid

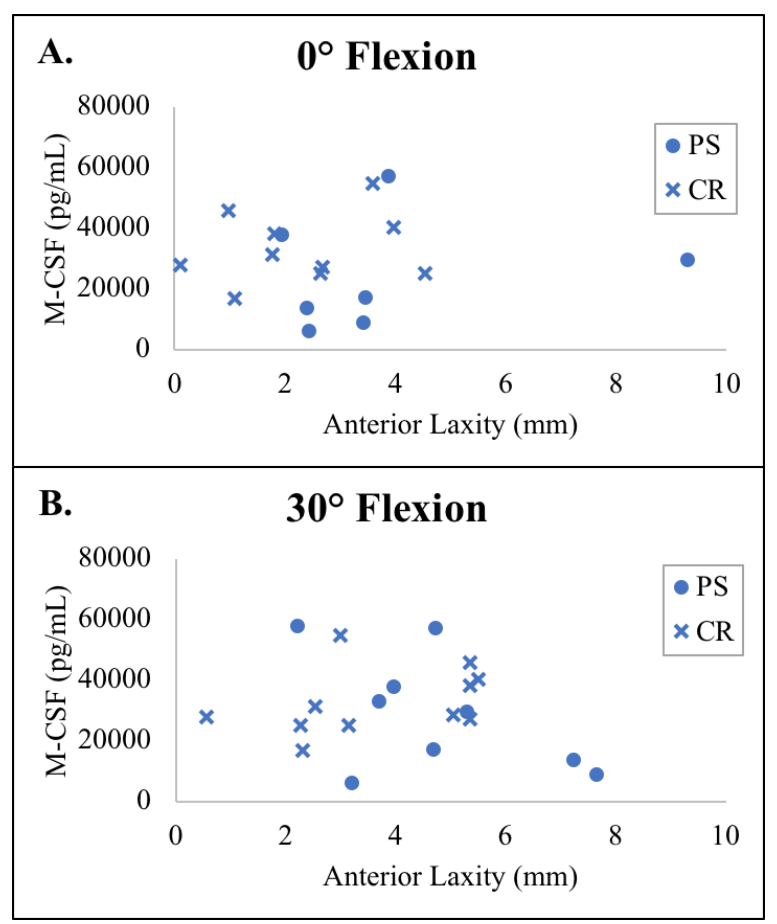

Figure C-45. Relationship between anterior laxity at (A) $0^{\circ}$ and (B) $30^{\circ}$ of flexion and M-CSF concentrations in synovial fluid 


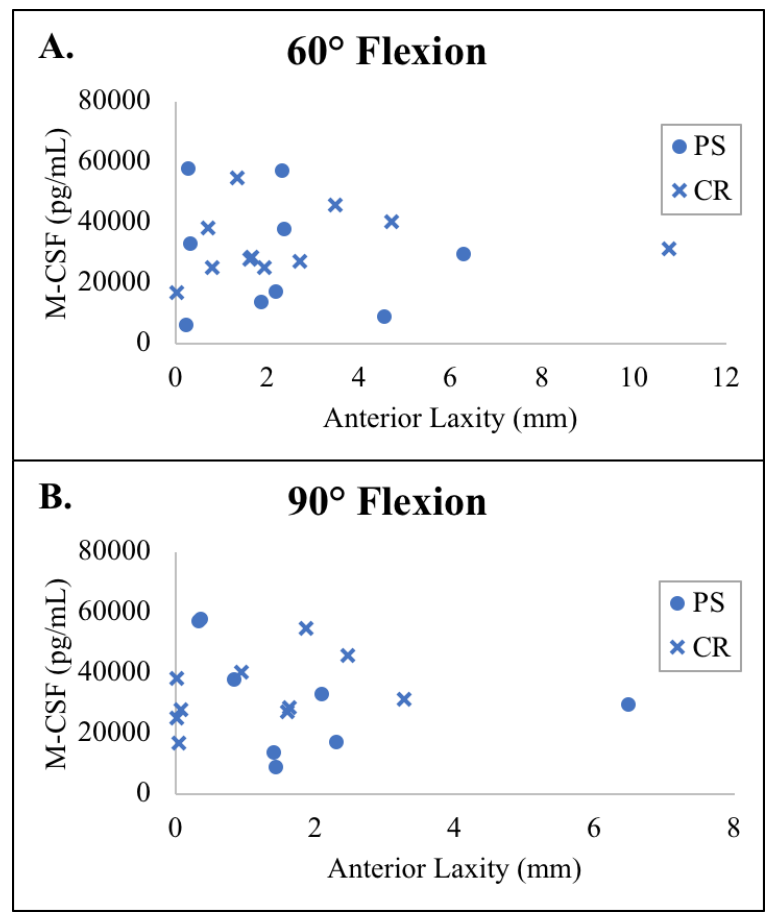

Figure C-46. Relationship between anterior laxity at (A) $60^{\circ}$ and (B) $90^{\circ}$ of flexion and M-CSF concentrations in synovial fluid

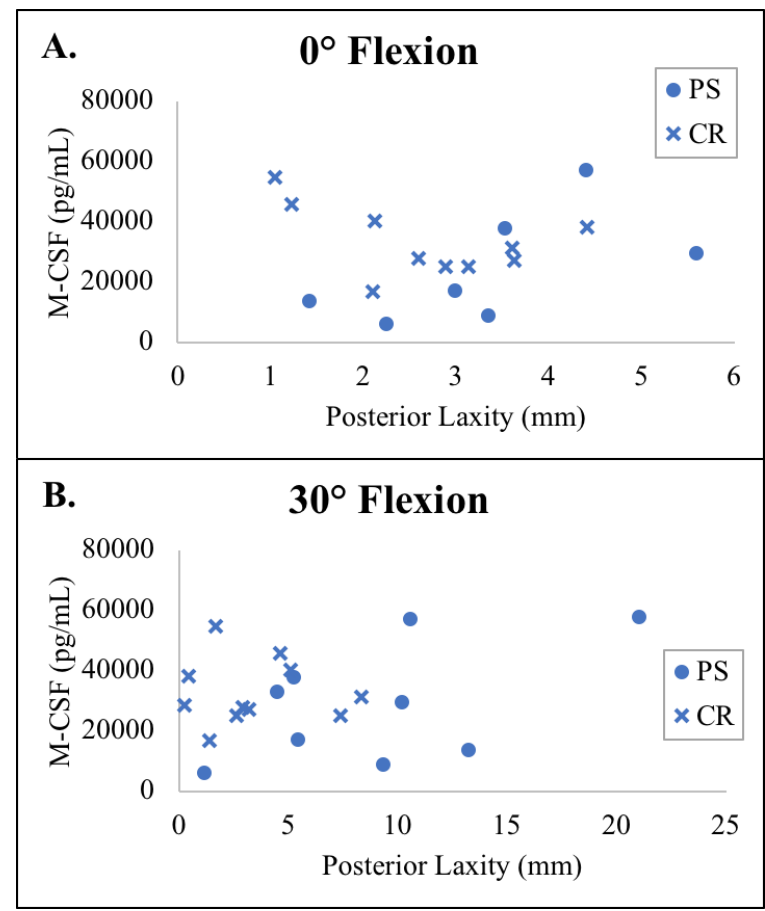

Figure C-47. Relationship between posterior laxity at (A) $0^{\circ}$ and (B) $30^{\circ}$ of flexion and M-CSF concentrations in synovial fluid 


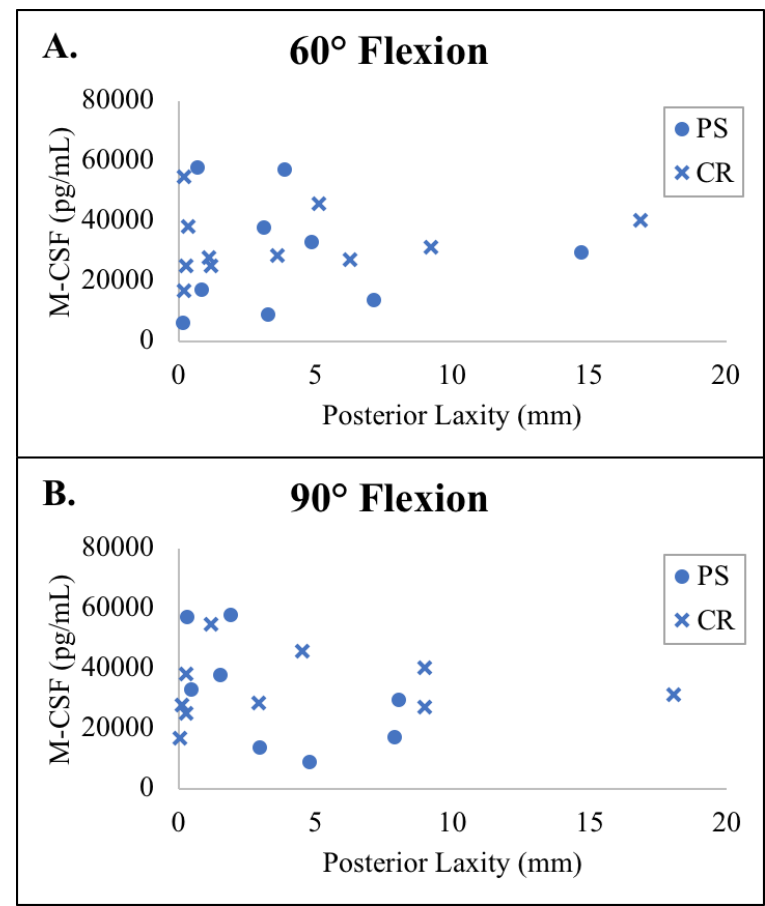

Figure C-48. Relationship between posterior laxity at (A) $60^{\circ}$ and (B) $90^{\circ}$ of flexion and M-CSF concentrations in synovial fluid 


\section{APPENDIX D. CORRELATIONS BETWEEN TISSUE METAL CONCENTRATIONS AND INFLAMMATOR CYTOKINES}

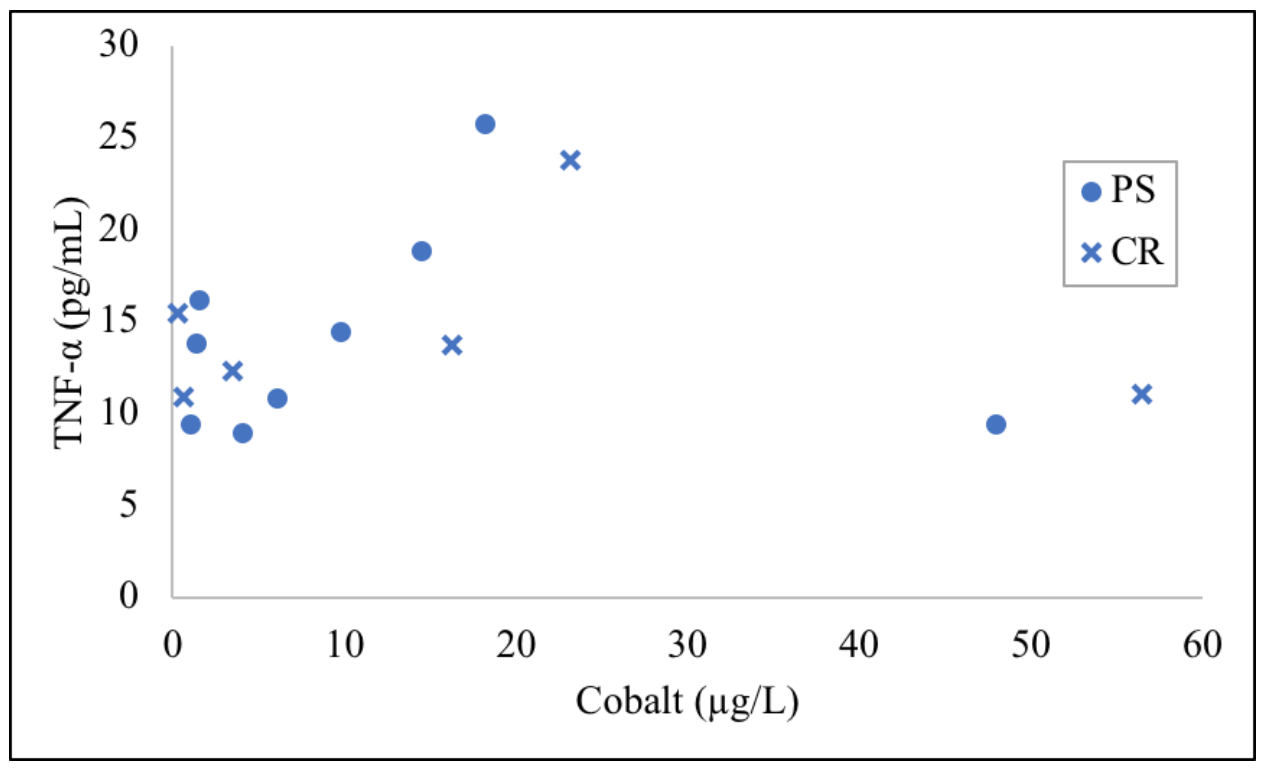

Figure D-1. Relationship between Co concentrations in periprosthetic tissue and TNF- $\alpha$ concentrations in synovial fluid

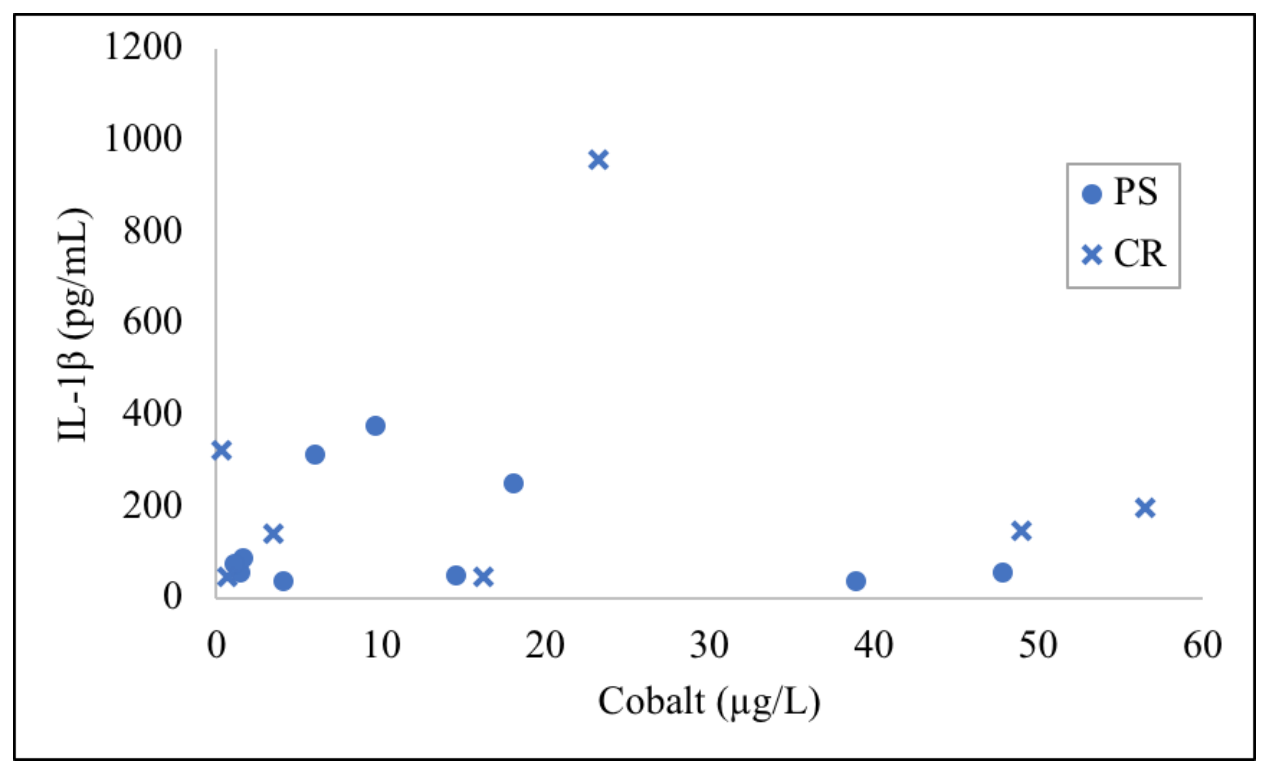

Figure D-2. Relationship between Co concentrations in periprosthetic tissue and IL-1 $\beta$ concentrations in synovial fluid 


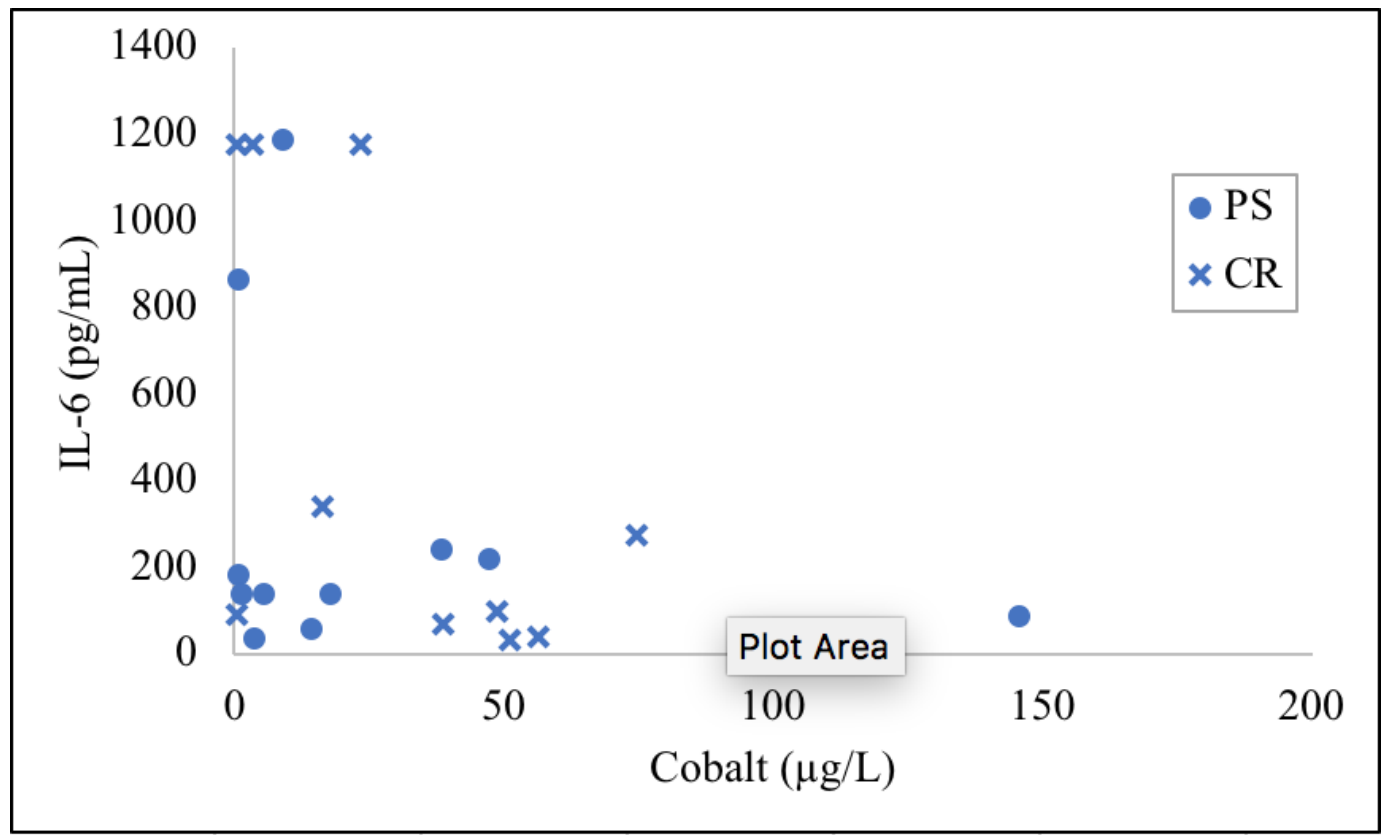

Figure D-3. Relationship between Co concentrations in periprosthetic tissue and IL-6 concentrations in synovial fluid

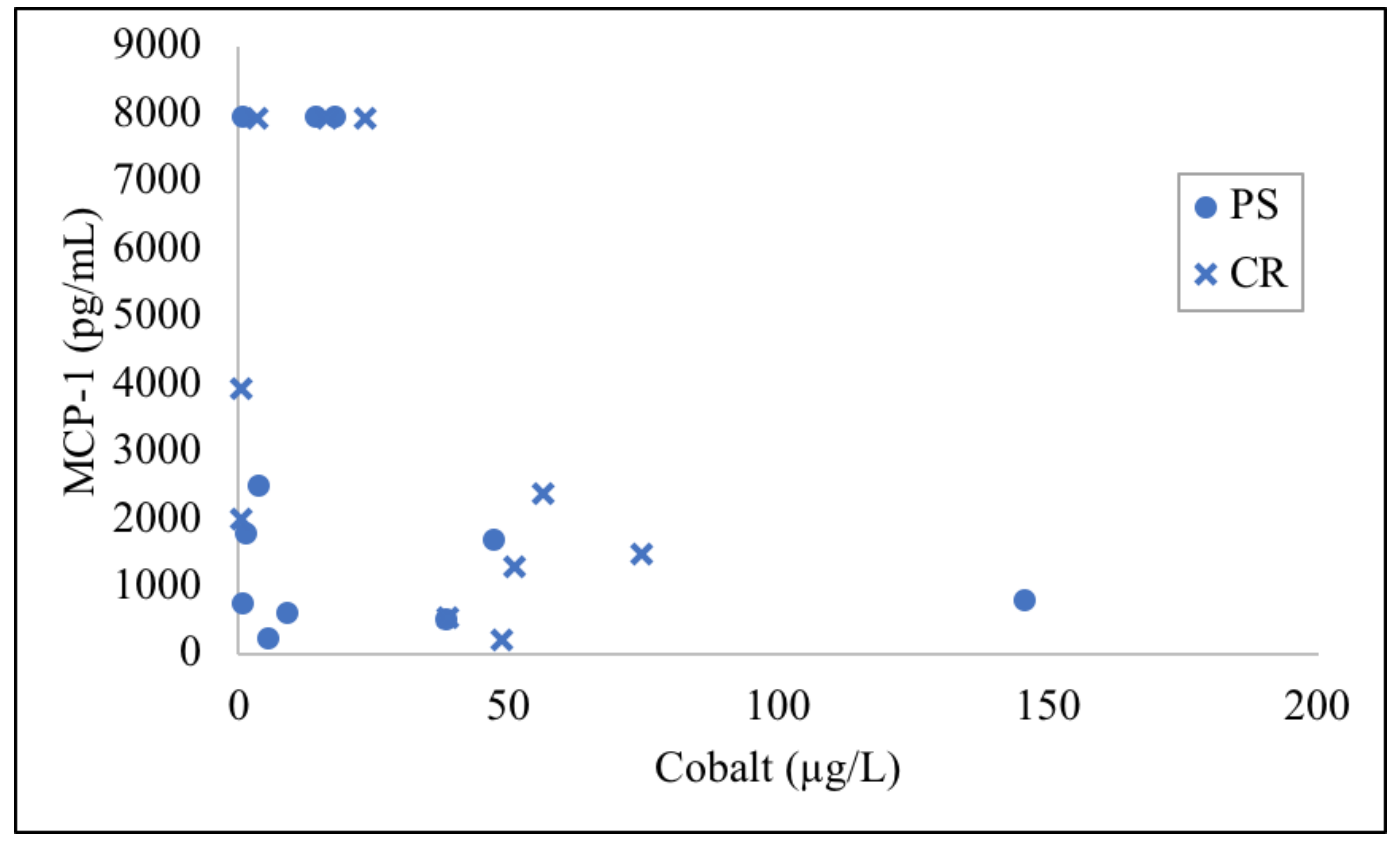

Figure D-4. Relationship between Co concentrations in periprosthetic tissue and MCP-1 concentrations in synovial fluid 


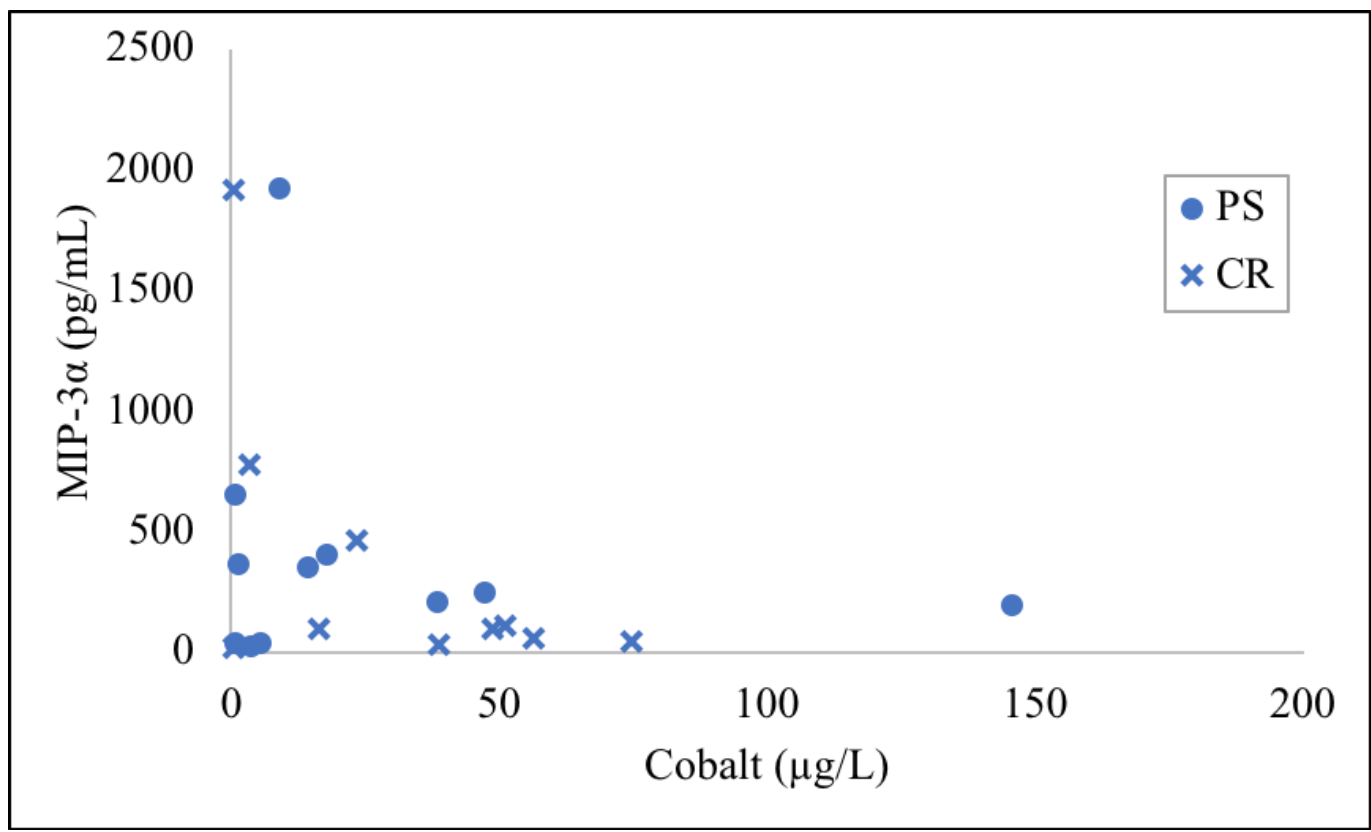

Figure D-5. Relationship between Co concentrations in periprosthetic tissue and MIP-3 $\alpha$ concentrations in synovial fluid

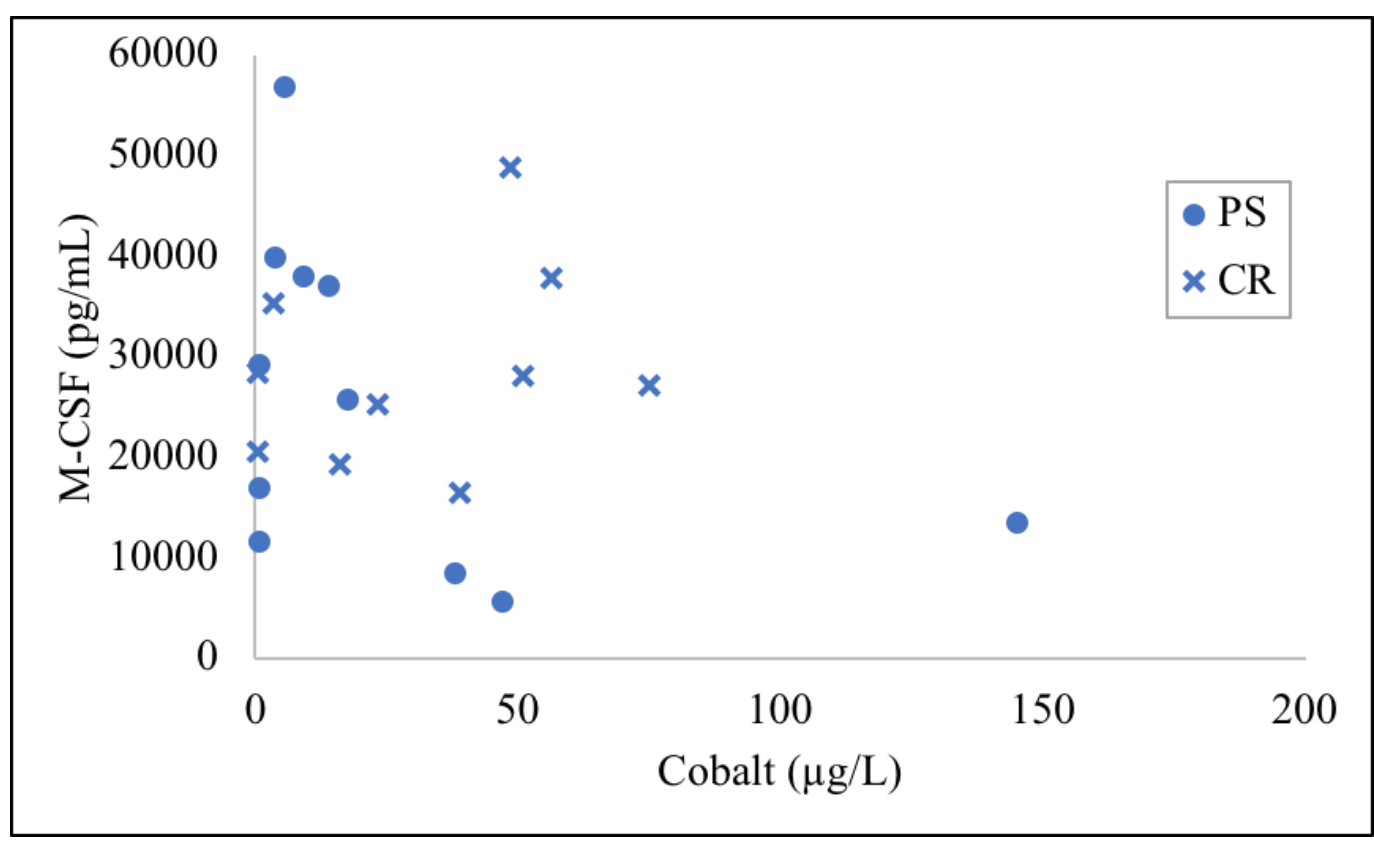

Figure D-6. Relationship between Co concentrations in periprosthetic tissue and M-CSF concentrations in synovial fluid 


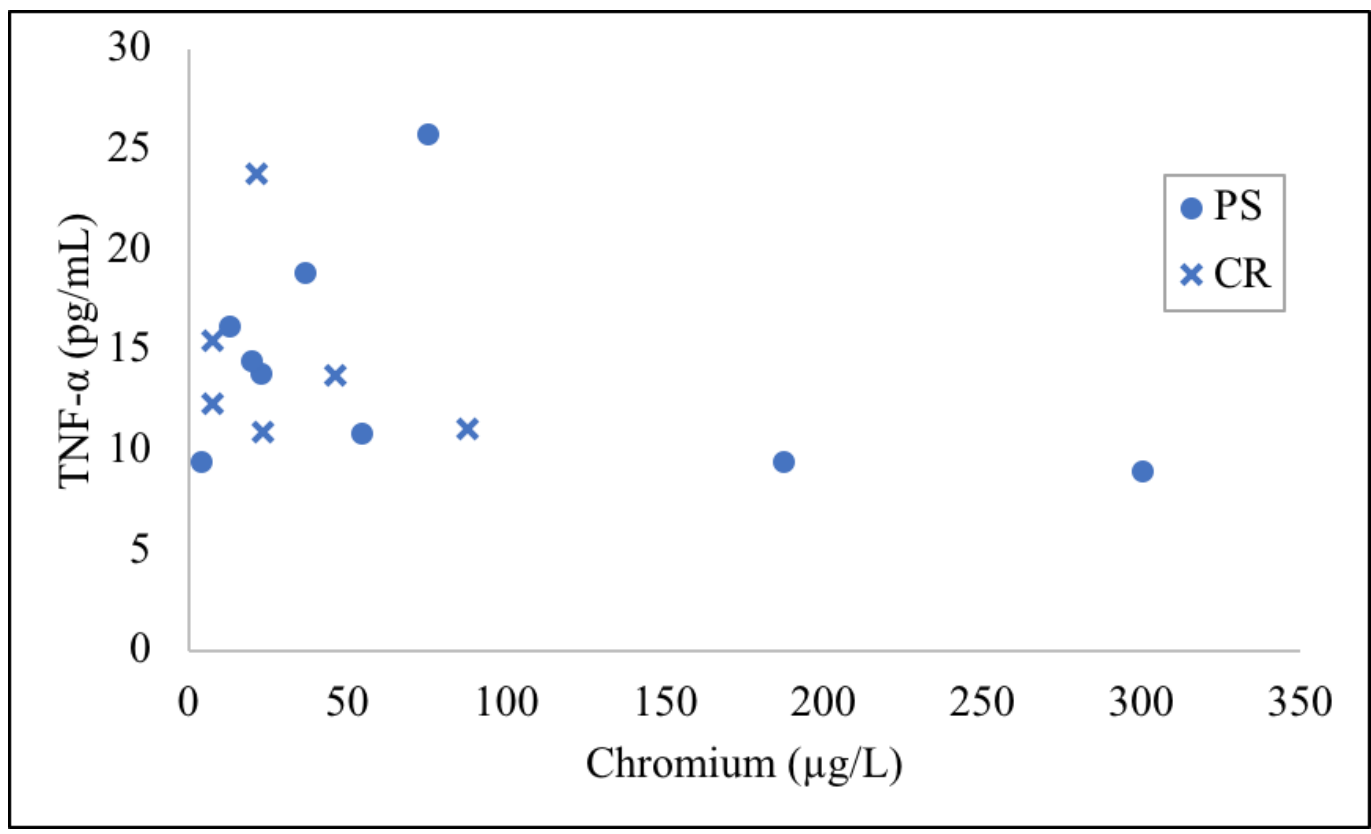

Figure D-7. Relationship between $\mathrm{Cr}$ concentrations in periprosthetic tissue and TNF- $\alpha$ concentrations in synovial fluid

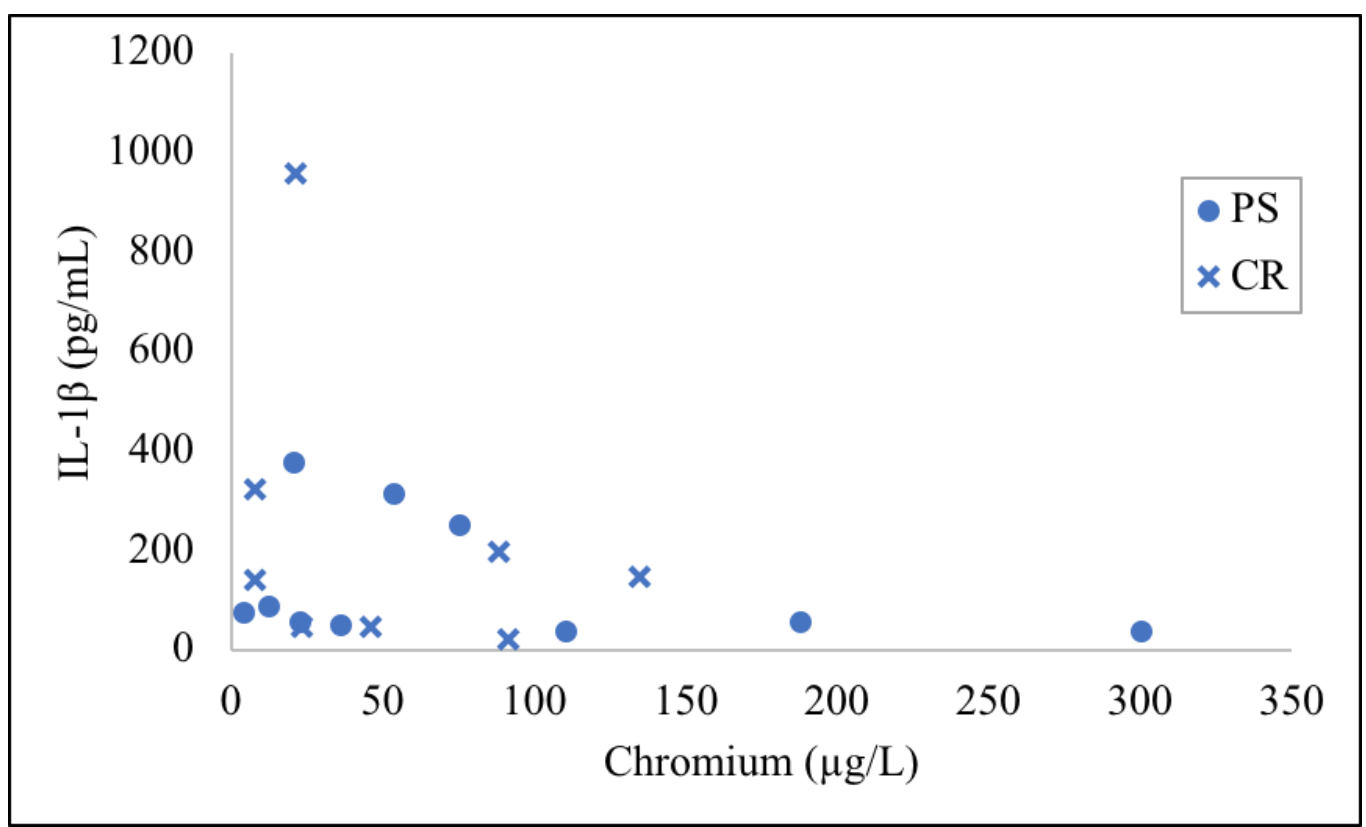

Figure D-8. Relationship between $\mathrm{Cr}$ concentrations in periprosthetic tissue and IL-1 $\beta$ concentrations in synovial fluid 


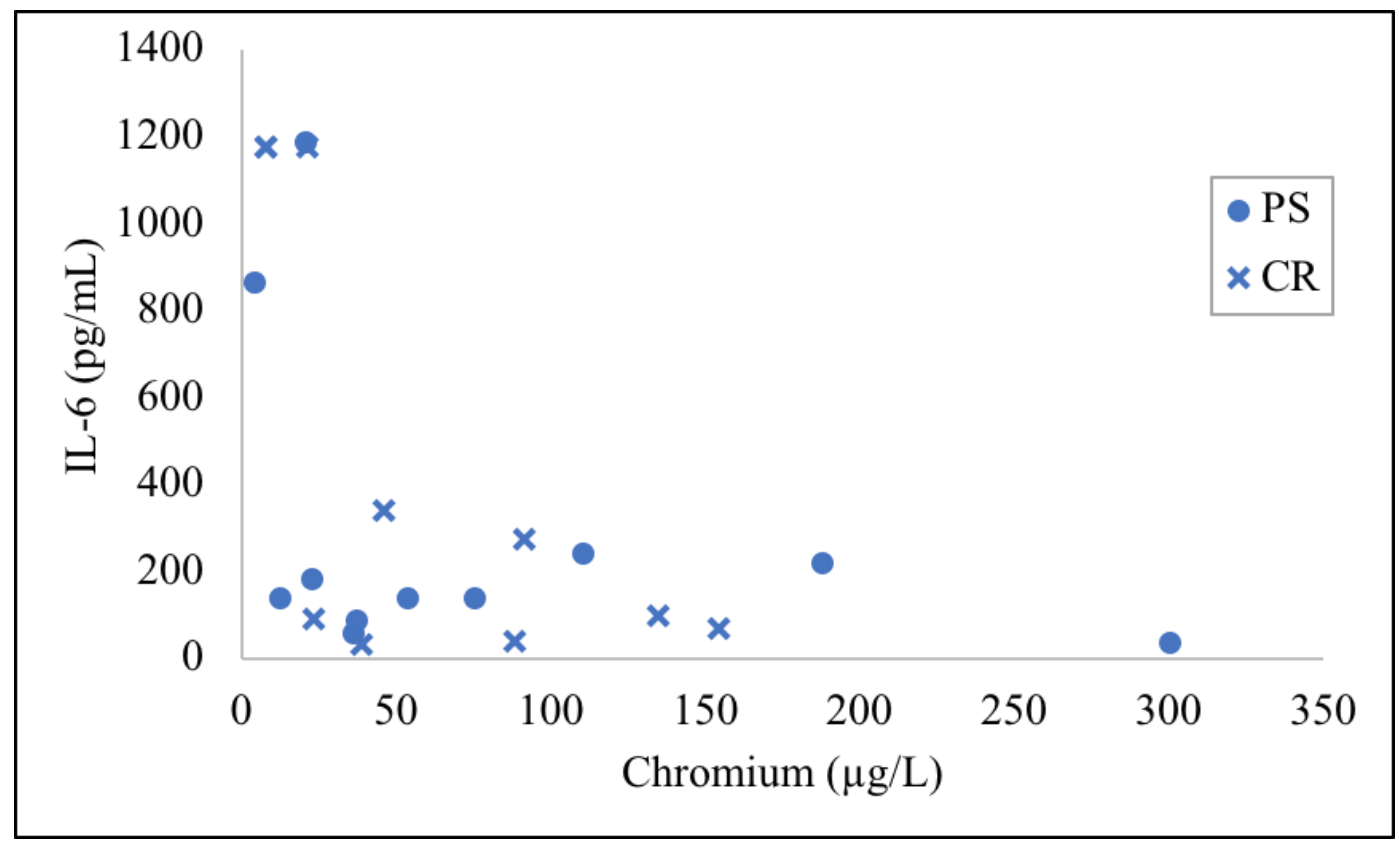

Figure D-9. Relationship between Cr concentrations in periprosthetic tissue and IL-6 concentrations in synovial fluid

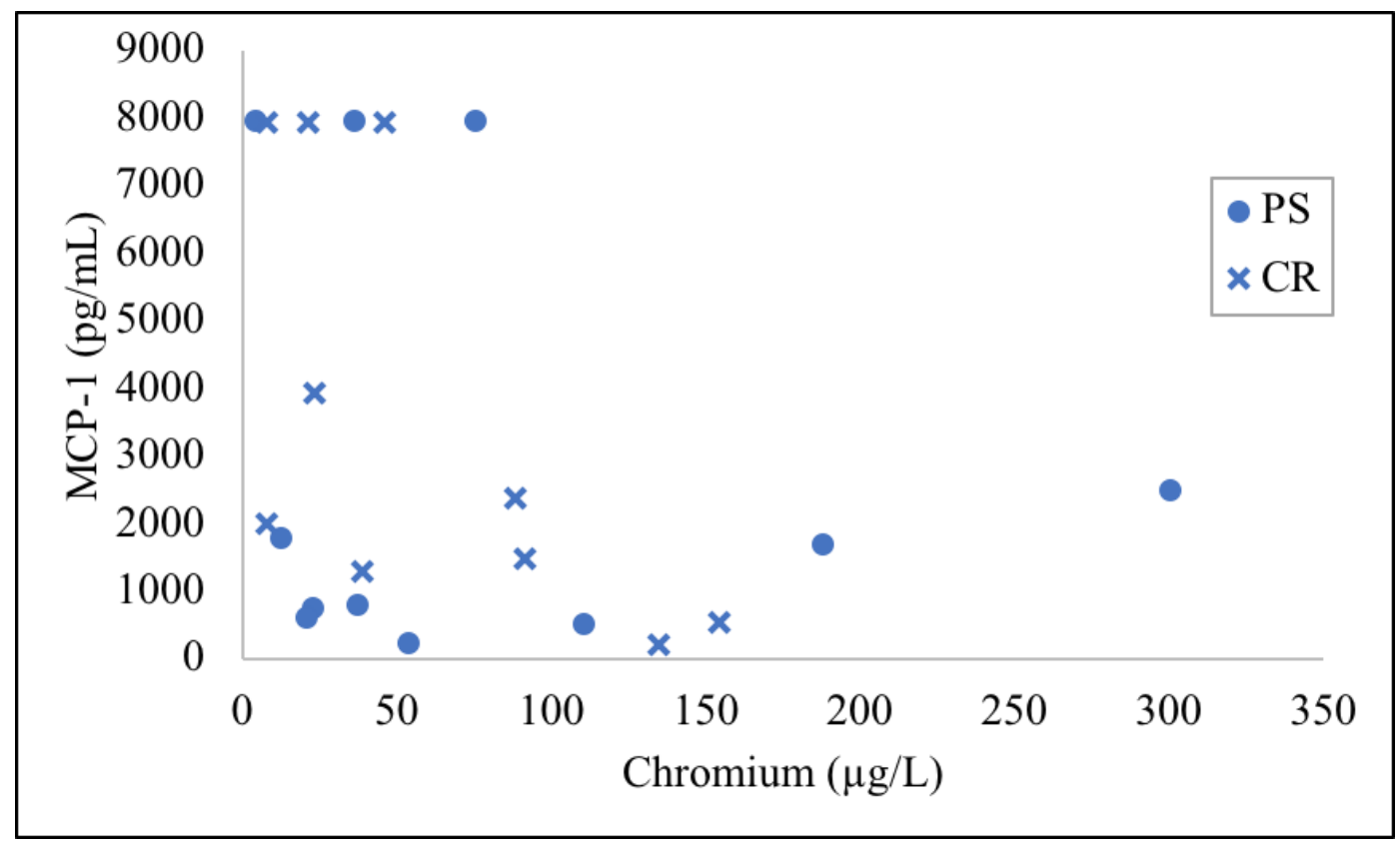

Figure D-10. Relationship between $\mathrm{Cr}$ concentrations in periprosthetic tissue and MCP-1 concentrations in synovial fluid 


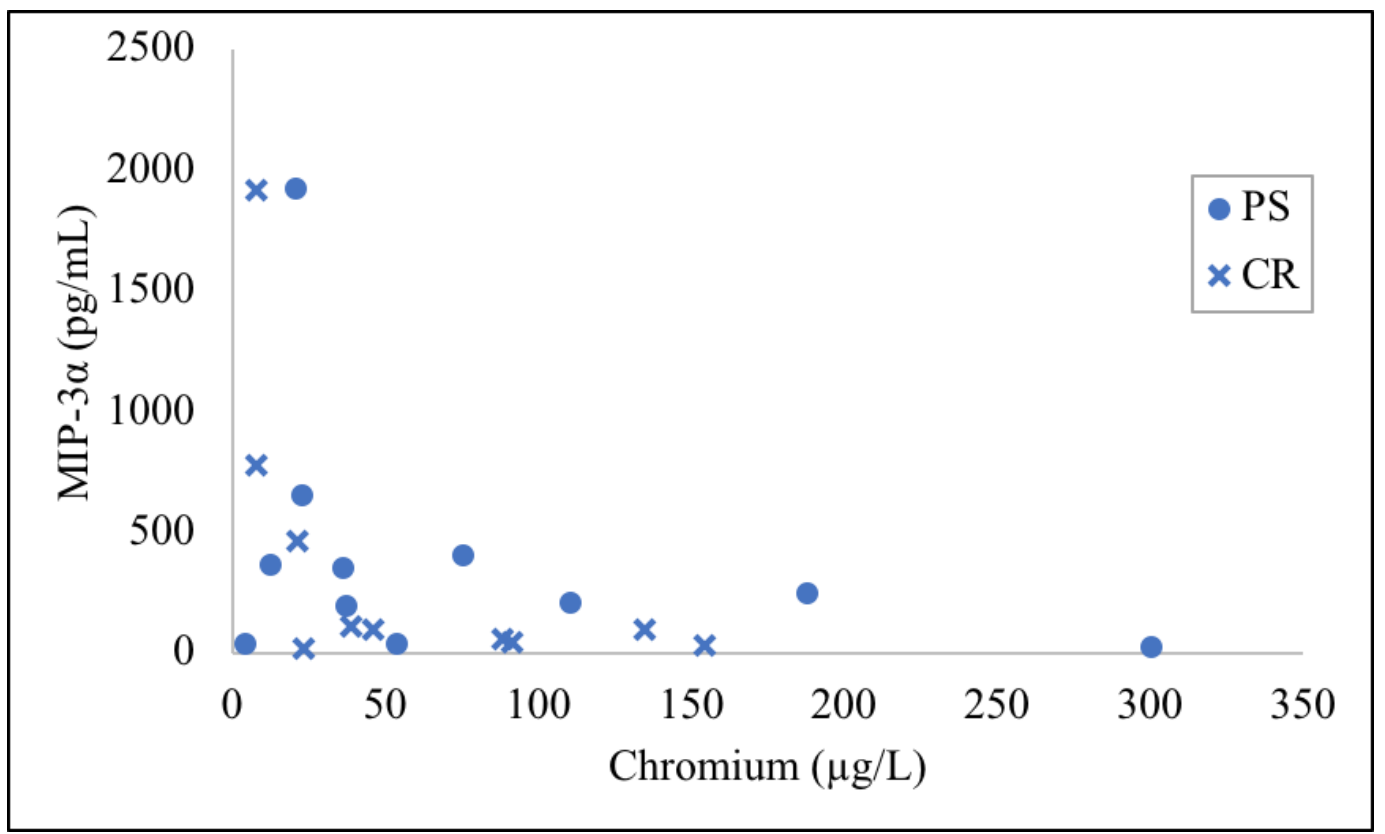

Figure D-11. Relationship between $\mathrm{Cr}$ concentrations in periprosthetic tissue and MIP-3 $\alpha$ concentrations in synovial fluid

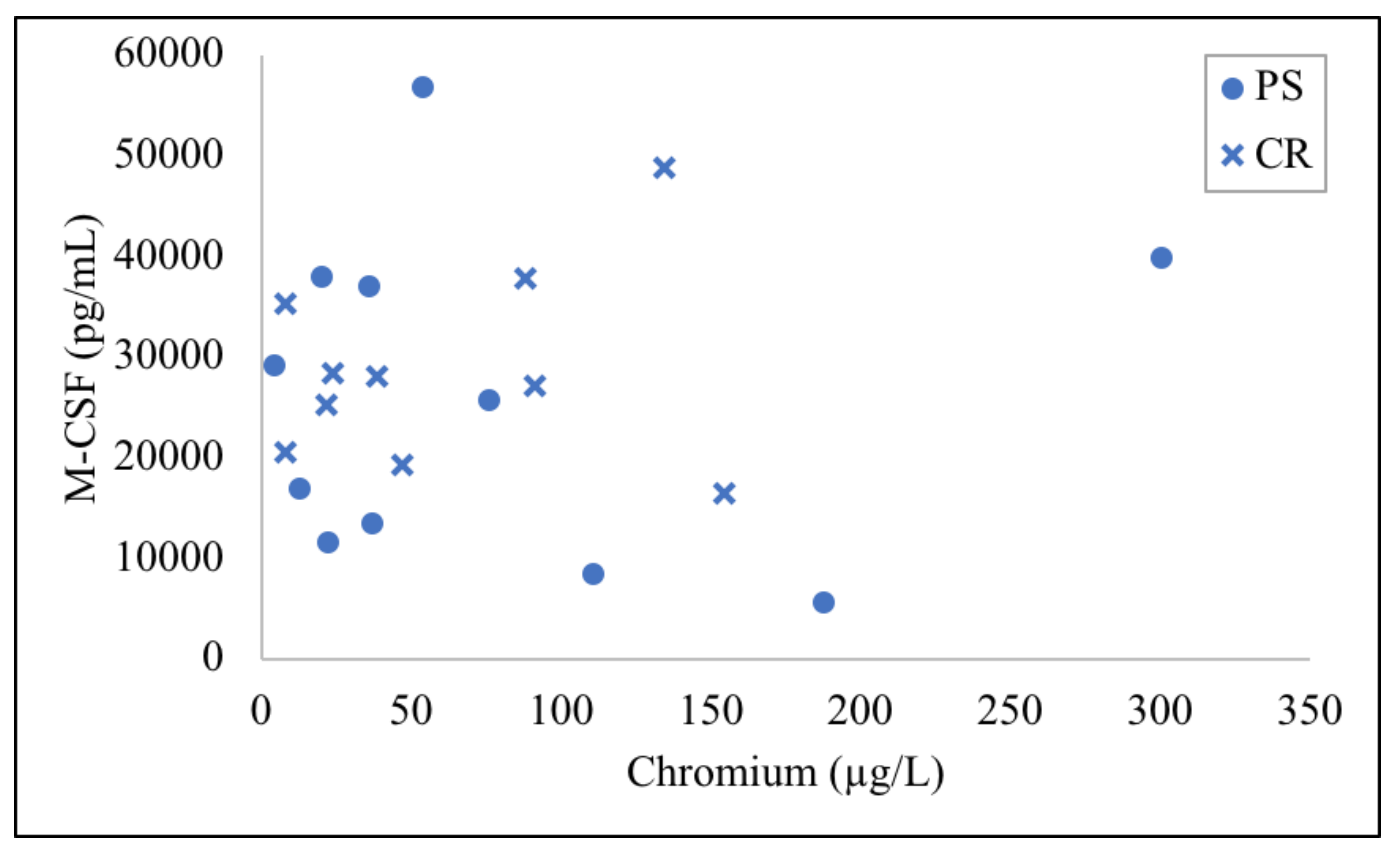

Figure D-12. Relationship between $\mathrm{Cr}$ concentrations in periprosthetic tissue and M-CSF concentrations in synovial fluid 


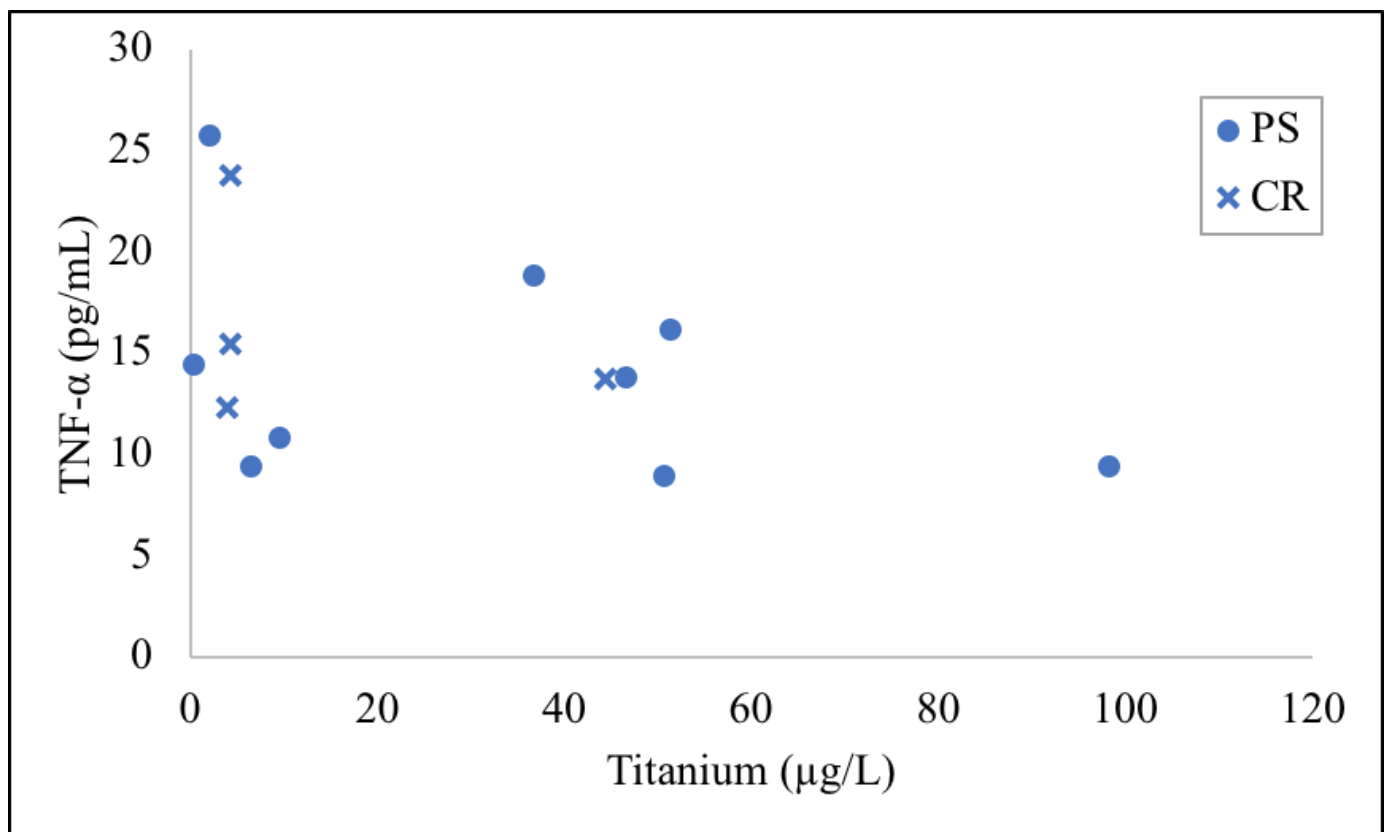

Figure D-13. Relationship between Ti concentrations in periprosthetic tissue and TNF- $\alpha$ concentrations in synovial fluid

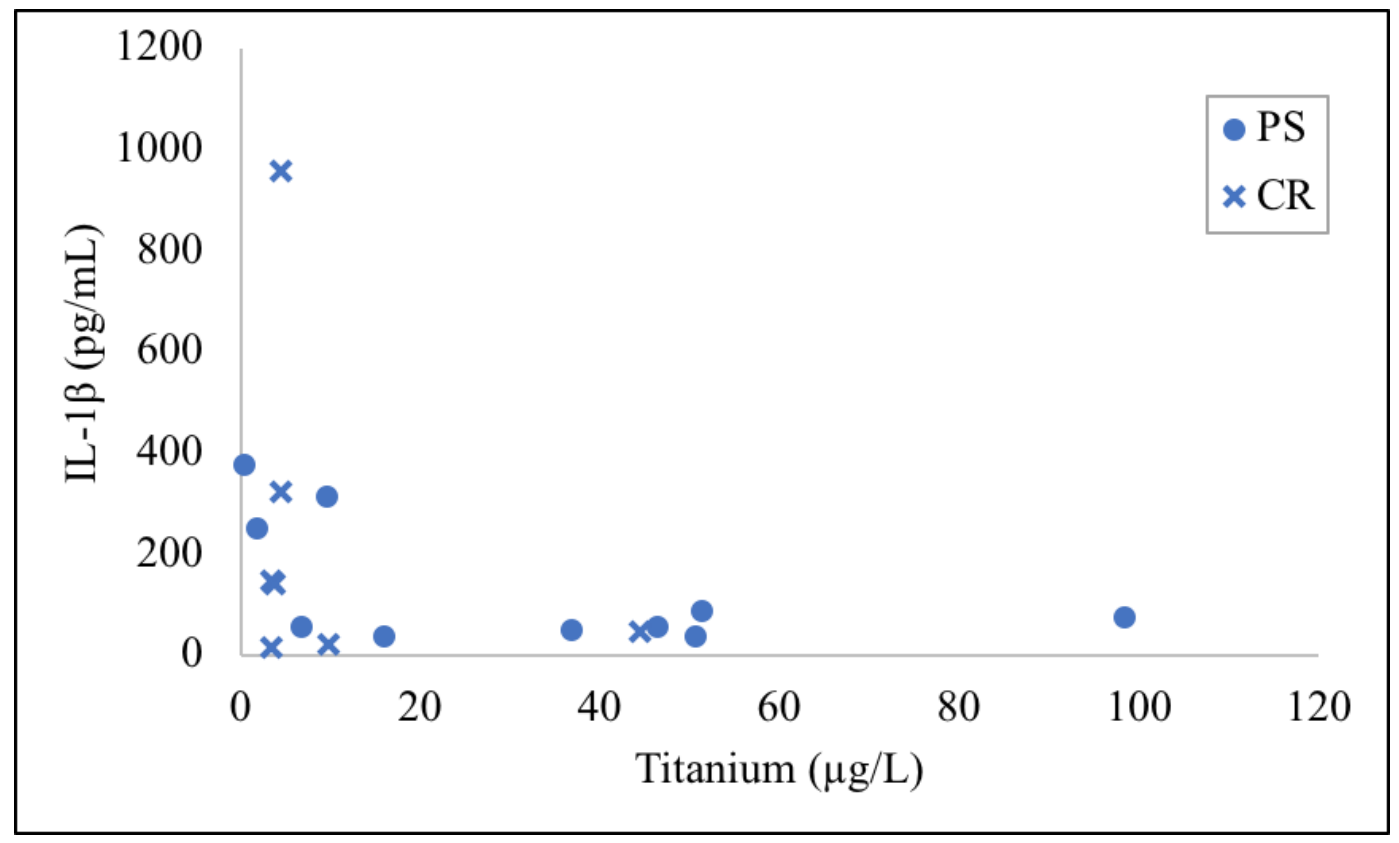

Figure D-14. Relationship between Ti concentrations in periprosthetic tissue and IL-1 $\beta$ concentrations in synovial fluid 


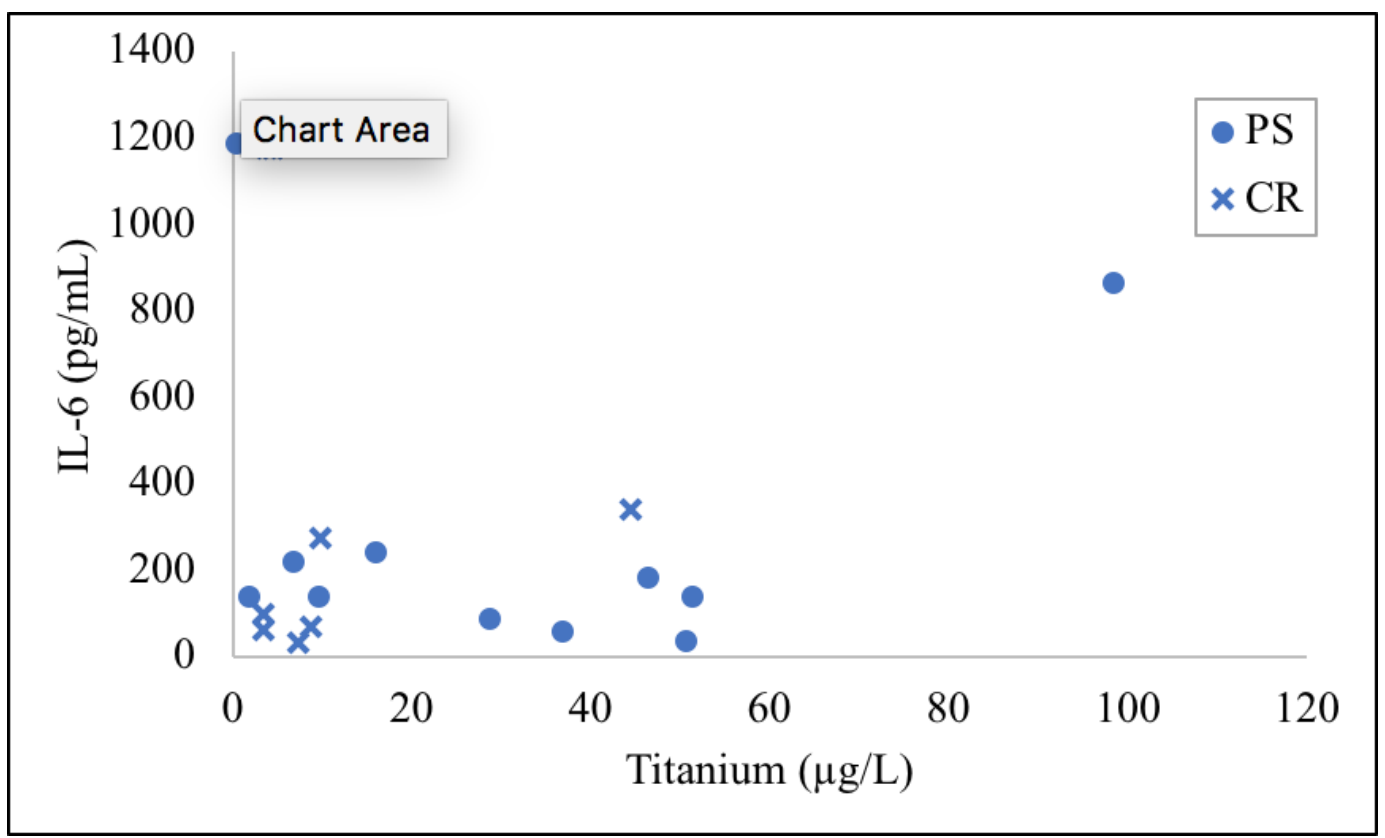

Figure D-15. Relationship between Ti concentrations in periprosthetic tissue and IL-6 concentrations in synovial fluid

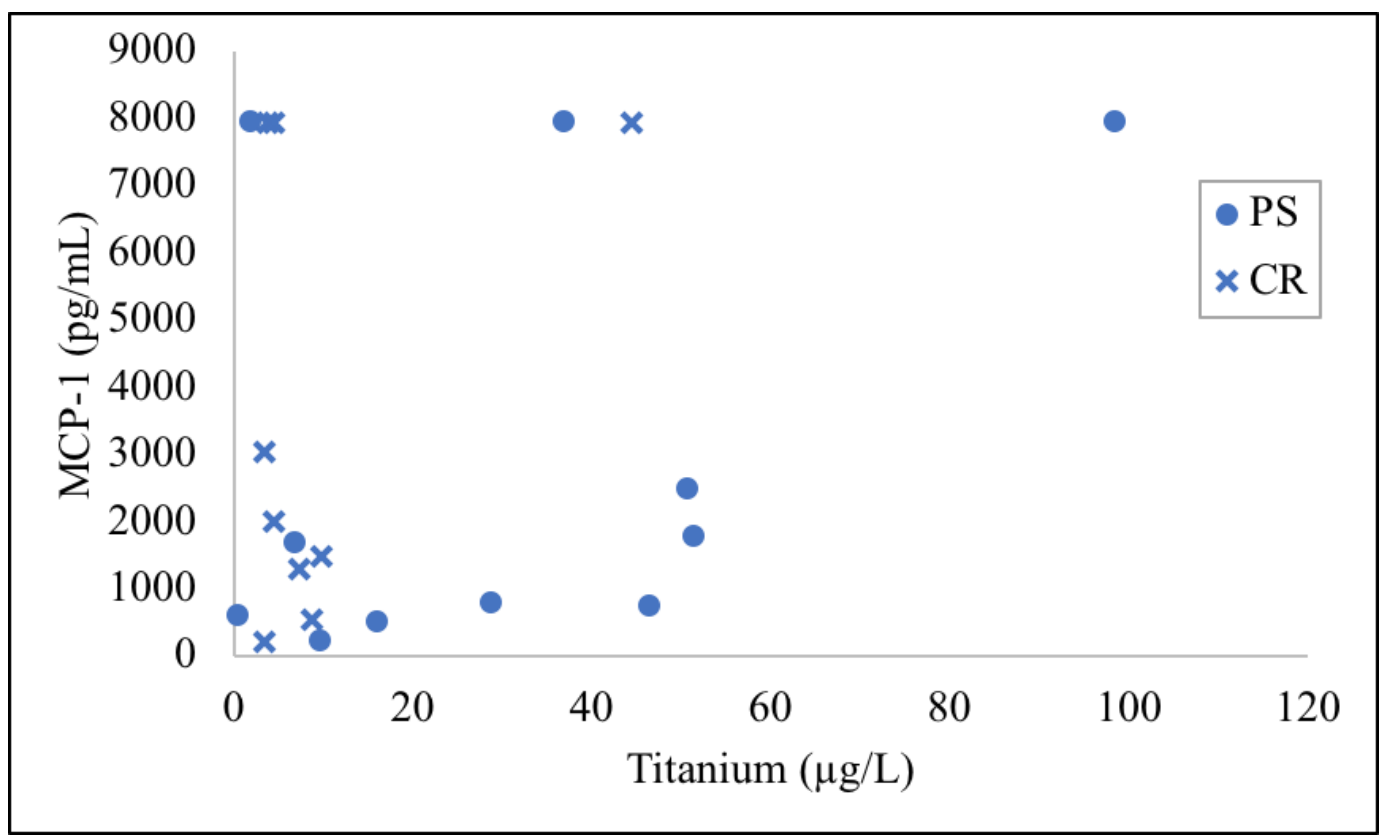

Figure D-16. Relationship between Ti concentrations in periprosthetic tissue and MCP-1 concentrations in synovial fluid 


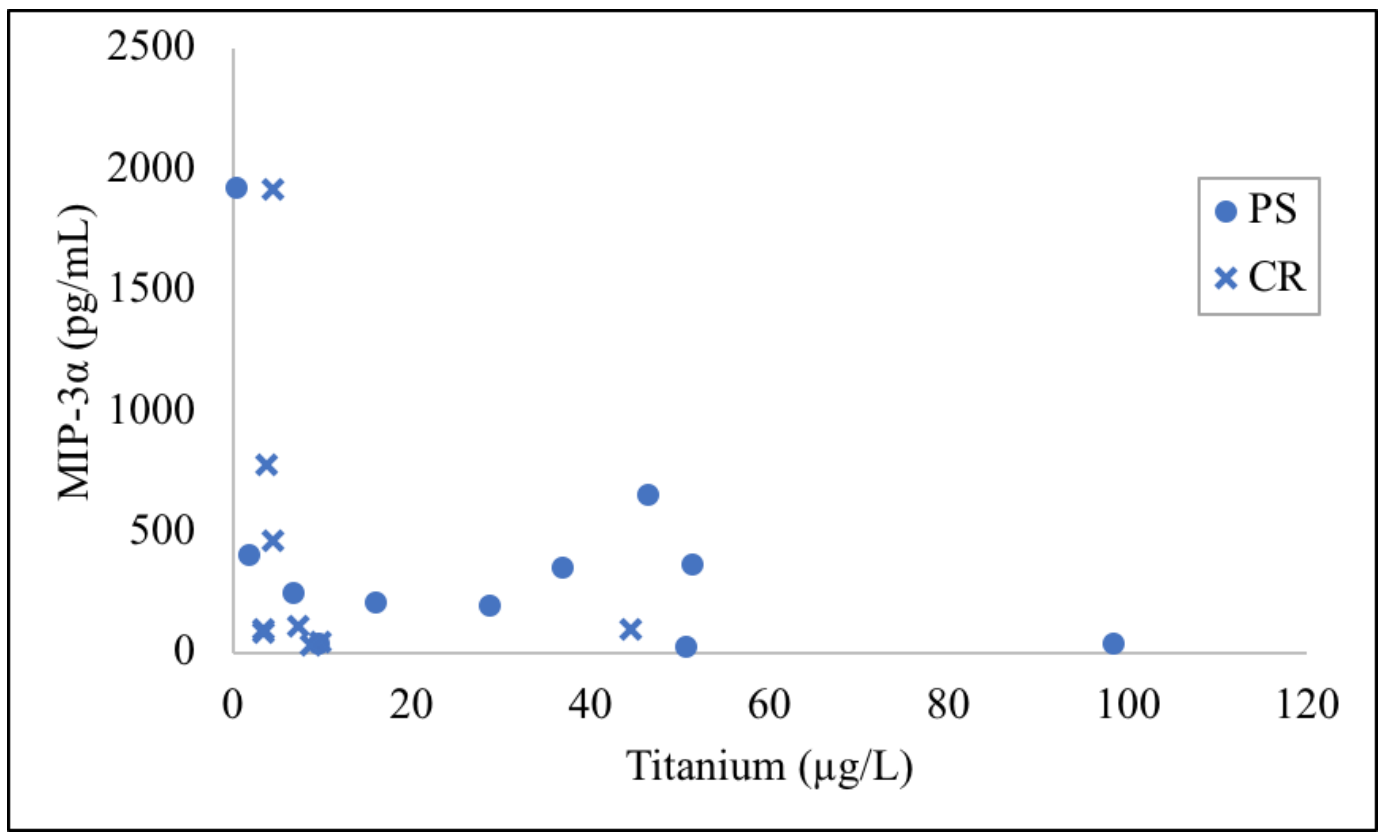

Figure D-17. Relationship between $\mathrm{Cr}$ concentrations in periprosthetic tissue and MIP-3 $\alpha$ concentrations in synovial fluid

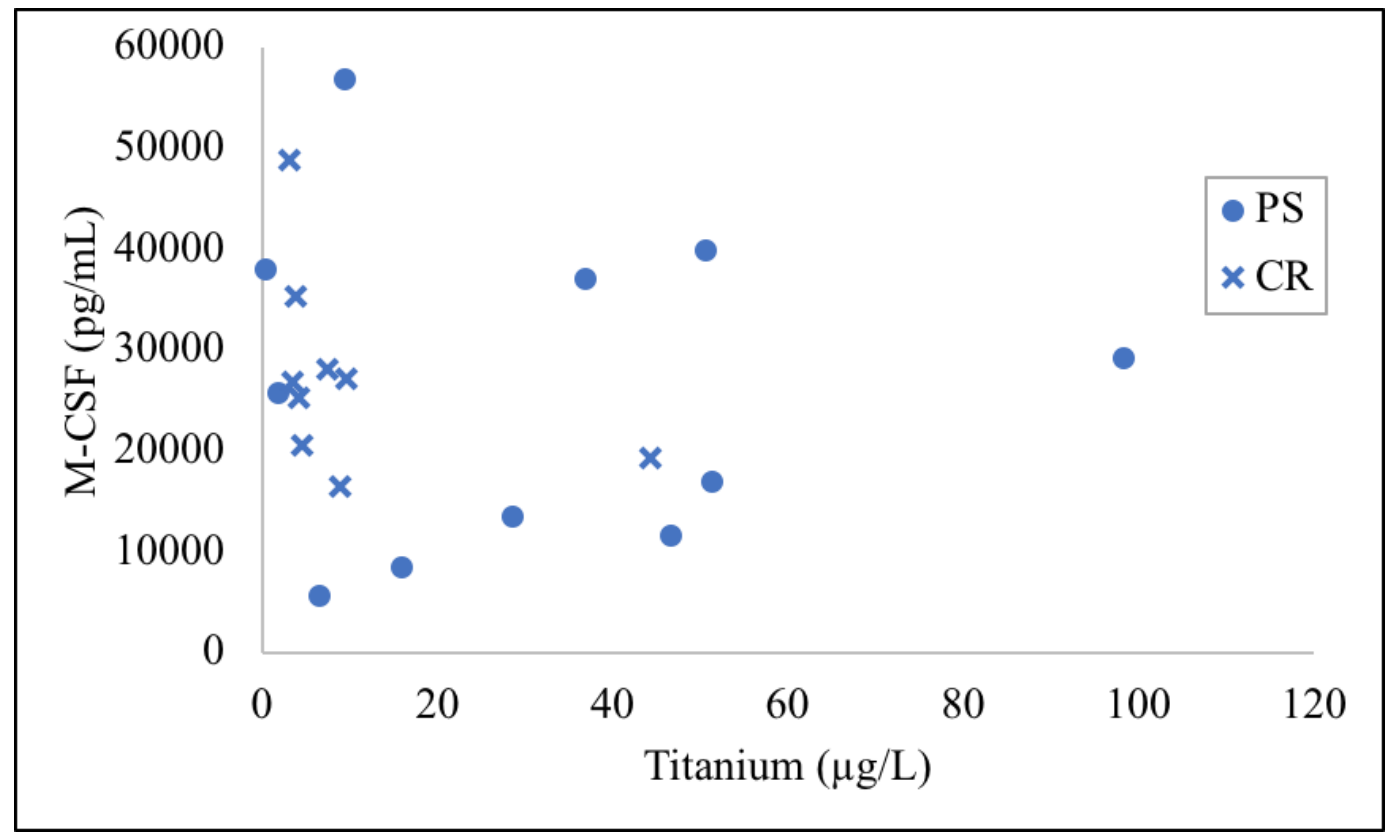

Figure D-18. Relationship between Ti concentrations in periprosthetic tissue and M-CSF concentrations in synovial fluid 


\section{VITA}

Meredith Riley Perkins was born in Memphis, TN in 1993. She attended University of Mississippi in Oxford, Mississippi where she earned a Bachelor of Science in Mechanical Engineering with a Specialization in Pre-Medicine in 2016. After graduation, she moved back to Memphis, Tennessee to begin her work as a graduate student under Dr. William Mihalko while obtaining her Master of Science in Biomedical Engineering from the Joint Program in Biomedical Engineering at the University of Tennessee Health Science Center and the University of Memphis. Meredith earned her Master of Science Degree in Biomedical Engineering with a concentration in biomechanics in May 2018. 